\title{
WestVirginiaUniversity
}

THE RESEARCH REPOSITORY @ WVU

Graduate Theses, Dissertations, and Problem Reports

2001

\section{Simulation of heavy-duty hybrid electric vehicles}

Anjali Devi Nennelli

West Virginia University

Follow this and additional works at: https://researchrepository.wvu.edu/etd

\section{Recommended Citation}

Nennelli, Anjali Devi, "Simulation of heavy-duty hybrid electric vehicles" (2001). Graduate Theses,

Dissertations, and Problem Reports. 1204.

https://researchrepository.wvu.edu/etd/1204

This Thesis is protected by copyright and/or related rights. It has been brought to you by the The Research Repository @ WVU with permission from the rights-holder(s). You are free to use this Thesis in any way that is permitted by the copyright and related rights legislation that applies to your use. For other uses you must obtain permission from the rights-holder(s) directly, unless additional rights are indicated by a Creative Commons license in the record and/ or on the work itself. This Thesis has been accepted for inclusion in WVU Graduate Theses, Dissertations, and Problem Reports collection by an authorized administrator of The Research Repository @ WVU. For more information, please contact researchrepository@mail.wvu.edu. 


\section{Simulation of Heavy-Duty Hybrid Electric Vehicles}

\section{Anjali Devi Nennelli}

Thesis submitted to

The College of Engineering and Mineral Resources

at

West Virginia University

in partial fulfillment of the requirements for the degree of

Master of Science

in

Mechanical Engineering

Nigel N. Clark, Ph.D., Chair

Parviz Famouri, Ph. D

Gregory J. Thompson, Ph. D

Department of Mechanical and Aerospace Engineering

Morgantown, West Virginia 


\section{Abstract \\ Simulation of Heavy-Duty Hybrid Electric Vehicles \\ Anjali Devi Nennelli}

Recently hybrid electric vehicles have gained attention due to the increased concern about fuel economy and emissions. Global climate change has become an issue of primary concern. The ever-depleting natural resources of oil have made people to think of the alternative ways of transportation. Though hybrids do not completely rule out the usage of crude oil, they decrease its usage to a considerable extent. Hybrid electric vehicles (HEVs) have electric energy storage systems such as batteries, ultra capacitors, or flywheels and power units such as an internal combustion engine (ICE) or a fuel cell. These vehicles show great potential for use in a wide variety of driving situations, but the optimization of components and control strategies is quite complex.

Microsoft Excel was used to simulate the driving conditions of heavy-duty hybrid electric vehicles over various driving cycles in an attempt to optimize their design and control. Simulations were also run in a computer based vehicle simulation package called ADvanced VehIcle SimulatOR (ADVISOR). The fuel economy data thus obtained from the thesis simulation models and simulations run from ADVISOR were compared with those of the in-use heavy-duty hybrid electric vehicles. For this the drive cycles are modified to represent realistic expectations of the dynamic performance of vehicles. The cycles used are "Central Business District" cycle (CBD), "Urban Dynamometer Driving Schedule" cycle (UDDS), "Manhattan" cycle and "City Suburban Heavy Vehicle Cycle" (CSHVC). The vehicle considered for simulations was a transit bus, which is a series HEV. The series HEV is propelled solely by electric motors with energy coming from batteries and an alternator driven by an ICE. The simulation model is based on power 
requirements for the vehicle taking into account engine, battery, and driveline efficiencies. The control strategy forces the engine to run at a fixed percentage of the power required at the wheels, also taking into consideration battery state of charge correction factor.

Further emissions were also modeled for predicting NOx, as it is one of the significant emissions. NOx emissions were predicted as a function of $\mathrm{CO}_{2}$. The results are quite compatible with those of the in-use hybrid electric vehicles. The fuel economy of the thesis simulation model varies by $18.5 \%$ over CBD cycle. Fuel economy of ADVISOR model varies by $11 \%$ with respect to actual in use heavy-duty vehicles over Manhattan cycle. This is due to the differences in control strategies and efficiencies of various components used. 


\section{Acknowledgements}

It gives me immense pleasure to wholeheartedly thank my research advisor at West Virginia University, Dr. Nigel Clark. I would like to thank him for his incessant support, motivation and most importantly patience. His guidance and encouragement is a large part in making all this possible.

I would also like to extend my special gratitude to Dr. Scott Wayne, Dr. Parviz Famouri and Dr. Gregory Thompson for their willingness to serve on my graduate committee and more importantly for their support and valuable suggestions.

I wouldn't have had such a wonderful experience at West Virginia University without the support and the fun with my friends. I am thankful to them for all their support during my Masters.

I accord my highest respects to my beloved parents, my brother and my sisters for their support, love and encouragement throughout my life. 


\section{Table of Contents}

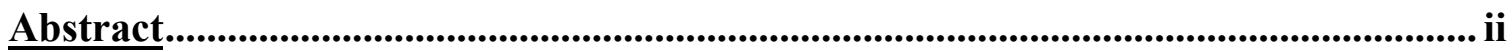

Acknowledgements ................................................................................................................... iv

Table of Contents

Index of Figures................................................................................................................... vii

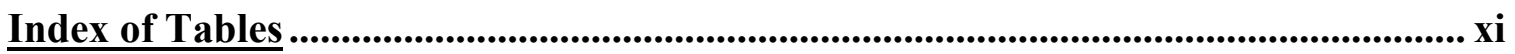

Nomenclature

1. Introduction and Objectives .................................................................................................... 1

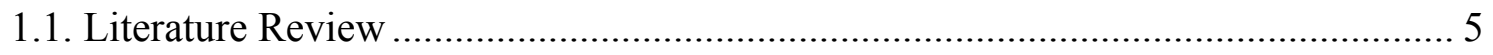

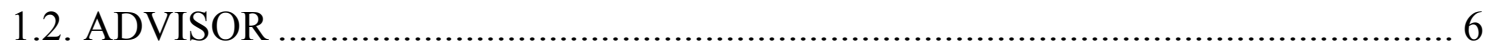

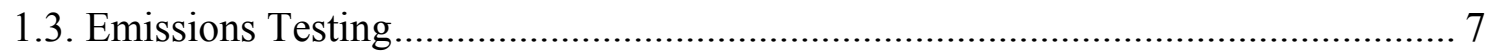

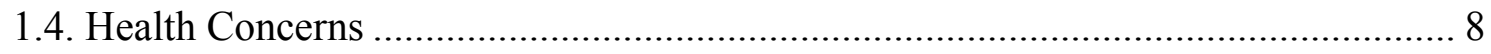

2. Types of Vehicle Layouts........................................................................................................ 12

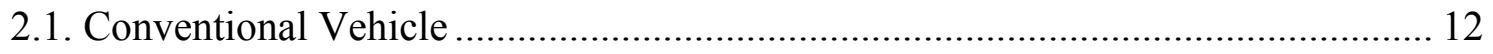

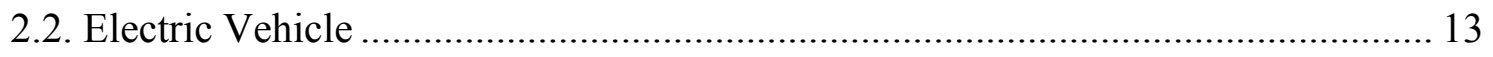

2.3. Series Hybrid Electric Vehicle …………………................................................... 14

2.4. Parallel Hybrid Electric Vehicle............................................................................ 15

2.5. Combination Hybrid Electric Vehicle .................................................................. 16

3. Drive Cycles ............................................................................................................................. 19

3.1. Power Consumed by a Vehicle.............................................................................. 25

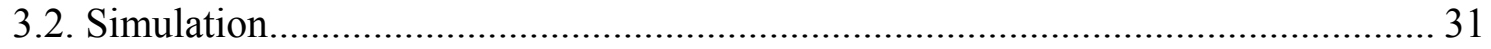

3.2.1. Series Control Strategy ................................................................................ 31 


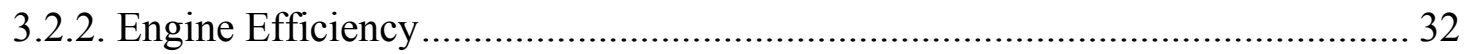

3.2.3. Battery Model and Simulation ............................................................... 33

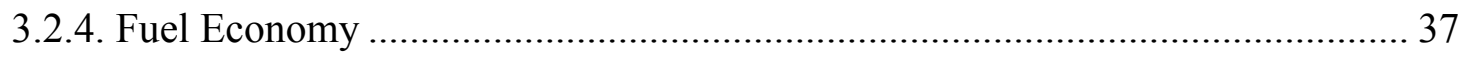

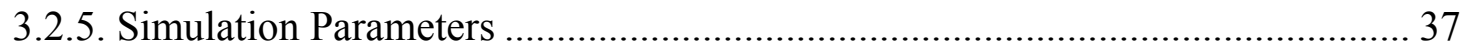

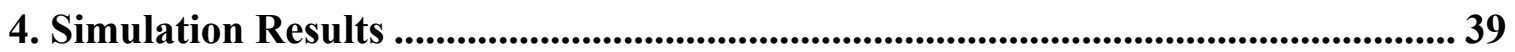

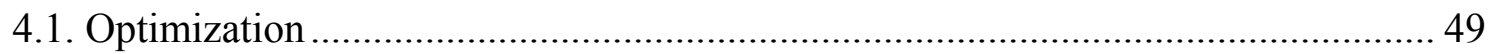

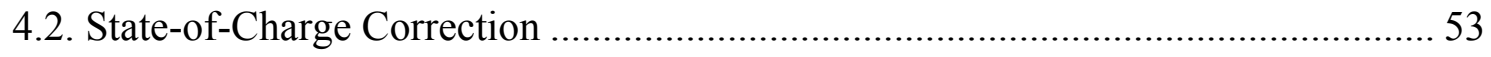

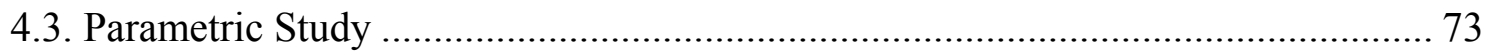

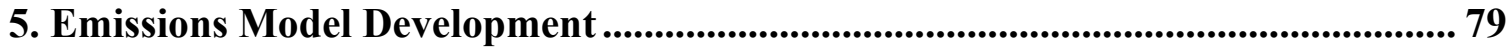

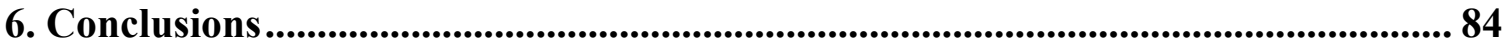

References............................................................................................................................ 85

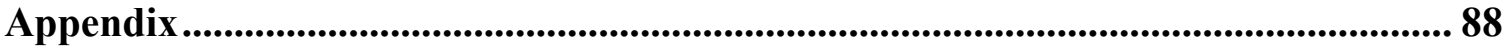




\section{Index of Figures}

Figure 1: Layout of Conventional Vehicle [6] ......................................................... 13

Figure 2: Layout of Electric Vehicle [6].................................................................... 14

Figure 3: Layout of Series HEV with power flowing into the ICE while coasting down the hill, engine acting as pump................................................................................ 14

Figure 4: HEV with batteries and ICE connected in parallel ...................................... 16

Figure 5: Layout for a Series-Parallel HEV [6] ............................................................... 17

Figure 6: Layout for a Planetary Combination HEV [6] ................................................ 18

Figure 7: CBD cycle consists of 14 peaks and has a maximum speed of $20 \mathrm{mph} . . . . .20$

Figure 8: Speed vs Time trace for UDDS cycle with an average speed of $18.86 \mathbf{~ m p h}$.

Figure 9: Manhattan cycle has 20 micro trips over a distance of 2.1 miles................ 22

Figure 10: Speed Vs Time trace for CSHVC with a maximum speed of $43.8 \mathrm{mph} . .23$

Figure 11: UDDS1 with highest peak at the end of cycle. ................................................ 24

Figure 12: Speed vs Time trace for UDDS2 cycle ............................................................. 24

Figure 13: Power required at wheels over CBD cycle ...................................................... 28

Figure 14: Power required at wheels over UDDS cycle..................................................... 29

Figure 15: Power required at wheels over CSHVC ............................................................ 29

Figure 16: Power required at wheels over Manhattan cycle .......................................... 30

Figure 17: Power required at wheels over UDDS1 cycle.................................................... 30

Figure 18: Power required at wheels over UDDS2 cycle................................................... 31

Figure 19: HEV on CBD cycle with auxiliary load for $\mathrm{C} 1=0.3$ and $\mathrm{SoC}$ at $90 \% \ldots . . . .42$

Figure 20: HEV on CBD cycle without auxiliary load for $\mathrm{C1}=0.4$ and $\mathrm{SoC}$ at $60 \% 42$ 
Figure 21: HEV on CSHVC with auxiliary load for $\mathrm{C} 1=0.3$ and $\mathrm{SoC}$ at $90 \% \ldots . . . . . .43$

Figure 22: HEV on CSHVC without auxiliary load for C1 0.4 and SoC at $60 \%$..... 44

Figure 23: HEV on Manhattan cycle with auxiliary load for $\mathrm{C} 1=0.3$ at $90 \%$ SoC .. 45

Figure 24: HEV on Manhattan cycle without auxiliary load for $\mathrm{C} 2=0.4$ at $60 \% \mathrm{SoC}$ 45

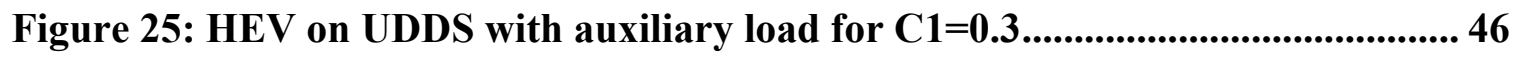

Figure 26: HEV on UDDS cycle without auxiliary load for $\mathrm{C} 1=0.4$ and $\mathrm{SoC}$ at $60 \%$

Figure 27: HEV on UDDS1 cycle with auxiliary load for $\mathrm{C} 1=0.3$ and SoC at $90 \% .47$

Figure 28: HEV on UDDS1 cycle without auxiliary load for $\mathrm{C} 1=0.4$ showing SoC at $60 \%$ 48

Figure 29: HEV on UDDS2 cycle with auxiliary load for $\mathrm{C} 1=0.4$ showing engine power and SoC 48

Figure 30: HEV on UDDS2 cycle without auxiliary load for $\mathrm{C} 1=0.3$ showing engine power and SoC 49

Figure 31: HEV Fuel Economy on CBD cycle............................................................. 50

Figure 32: HEV Fuel Economy on Manhattan cycle..................................................50

Figure 33: HEV Fuel Economy on CSHVC ..................................................... 51

Figure 34: HEV Fuel Economy on UDDS cycle ................................................... 51

Figure 35: HEV Fuel Economy on UDDS1 cycle ......................................................... 52

Figure 36: HEV Fuel Economy on UDDS2 cycle ....................................................... 52

Figure 37: MPG of HEV without auxiliary load on CBD cycle at C1=0.4 .............. 61 
Figure 38: The points representing MPG at SoC $60 \%-60 \%$ and SoC $90 \%-90 \%$ overlap for a HEV over $\mathrm{CBD}$ cycle at $\mathrm{C} 1=0.3$

Figure 39: 6\% difference is observed for the points representing MPG of HEV at zero delta SoC and line intersecting SoC at $60 \%$ and at $90 \%$ over CSHVC at $\mathrm{C} 1=0.3$

Figure 40:-0.9\% difference is observed for the points representing MPG of HEV at zero delta SoC and line intersecting SoC at $60 \%$ \& at $90 \%$ over CSHVC at $\mathrm{C} 1=\mathbf{0 . 5}$ 64

Figure 41:-6\% difference is observed between the points representing MPG of HEV at zero delta SoC and line intersecting SoC at $60 \%$ \& at $90 \%$ over CSHVC at $\mathrm{C} 1=0.5$ 65

Figure 42: MPG of HEV with auxiliary load on Manhattan Cycle at $\mathrm{C1}=0.5$, with a difference of $6 \%$ between the MPG of zero delta SoC points and line intersecting SoC at $60 \%$ \& $\mathrm{SoC}$ at $90 \%$ 66

Figure 43: MPG of HEV without auxiliary load on UDDS cycle at $\mathrm{C} 1=0.4$, with a difference of $-27 \%$ between the MPG of zero delta SoC points and line intersecting SoC at $60 \%$ \& $\mathrm{SoC}$ at $90 \%$

Figure 44: MPG of HEV with auxiliary load on UDDS cycle at $\mathrm{C1}=0.5$, with $26 \%$ difference between the points at zero delta SoC and line intersecting SoC at $60 \%$ \& SoC at $90 \%$ 68

Figure 45: MPG of HEV without auxiliary load on UDDS1 cycle at $\mathrm{C} 1=0.3$, with $3.6 \%$ difference between the zero delta points and line intersecting SoC at $60 \%$ \& SoC at $90 \%$ 69 
Figure 46: MPG of HEV with auxiliary load on UDDS1 cycle at $\mathrm{C} 1=0.5$, with $2.4 \%$ difference between zero delta points and line intersecting SoC at $60 \%$ and SoC at $90 \%$

Figure 47: MPG of HEV on UDDS2 cycle without auxiliary load at $\mathrm{C} 1=0.5$, with $5.5 \%$ difference between zero delta SoC points and line intersecting SoC at $60 \%$ \& SoC at $90 \%$ 71

Figure 48: MPG of HEV on UDDS2 with auxiliary load for $\mathrm{C} 1=0.5$, with $7.3 \%$ difference between zero delta SoC points and line intersecting SoC at $60 \%$ \& SoC at $90 \%$ 72

Figure 49: Advisor simulation on UDDS cycle............................................................... 75

Figure 50: Advisor simulation on CSHVC ....................................................... 76

Figure 51: Advisor simulation on Manhattan cycle............................................... 77

Figure 52: Advisor simulation on CBD cycle .................................................................... 78

Figure 53: $\mathrm{NOx}$ vs $\mathrm{CO}_{2}$ for three consecutive runs on $\mathrm{CBD}$ cycle for vehicle 6353.. 81 Figure 54: NOx vs $\mathrm{CO}_{2}$ for three different runs on Manhattan cycle for vehicle 6353. 


\section{$\underline{\text { Index of Tables }}$}

Table 1: EPA Emissions Standards for Heavy-Duty Diesel Engines, g/bhp·hr [12]. 10

Table 2: California Emissions Standards for Heavy-Duty Diesel Engines, g/bhp·hr [12]

Table 3: Hawker Genesis G25EP Battery Properties. [18] ............................................. 35

Table 4:Simulation Parameters for Conventional Heavy Duty Vehicle...................... 38

Table 5:Simulation Parameters for Heavy-Duty Hybrid Electric Vehicle................. 38

Table 6: Fuel Economy for Conventional Vehicles............................................................. 40

Table 7: Fuel Economy from simulations for Series HEVs ............................................ 41

Table 8: SoC correction of HEV without auxiliary load on CBD cycle...................... 53

Table 9: SoC correction for HEV with auxiliary load over CBD cycle ....................... 54

Table 10: SoC correction for HEV without auxiliary load over CSHVC................... 54

Table 11: SoC correction for HEV with auxiliary load over CSHVC .........................55

Table 12: SoC correction for HEV without auxiliary load over Manhattan cycle ... 55

Table 13: SoC correction of HEV with auxiliary load over Manhattan cycle ........... 56

Table 14: SoC correction for HEV without auxiliary load over UDDS cycle ............ 56

Table 15: SoC correction for HEV with auxiliary load over UDDS cycle.................. 57

Table 16: SoC correction for HEV without auxiliary load over UDDS1 cycle .......... 57

Table 17: SoC correction for HEV with auxiliary load over UDDS1 cycle................ 58

Table 18: SoC correction for HEV without auxiliary load over UDDS2 cycle ........ 58 
Table 19: SoC correction for HEV with auxiliary load over UDDS2 cycle

Table 20: Parametric study on CBD cycle, values in the table represents the fuel

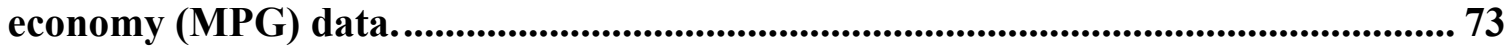

Table 21: Parametric study on Manhattan cycle, values in the table represents the fuel economy (MPG) data.

Table 22: Parametric study on CSHVC, values in the table represents the fuel economy (MPG) data.

Table 23: Parametric study on UDDS cycle, values in the table represents the fuel economy (MPG) data.

Table 24: Values of coefficients for the relation $\mathrm{NOx}$ as a function of $\mathrm{CO}_{2}$..............82

Table 25: SoC correction for HEV w/o auxiliary load over CBD cycle 89

Table 26: SoC correction for HEV with auxiliary load over CBD cycle 91

Table 27: SoC correction for HEV w/o auxiliary load over CSHVC 93

Table 28: SoC correction for HEV with auxiliary load over CSHVC 95

Table 29: SoC correction for HEV w/o auxiliary load over Manhattan cycle 97

Table 30: SoC correction for HEV with auxiliary load over Manhattan cycle 99

Table 31: SoC correction for HEV w/o auxiliary load over UDDS cycle 101

Table 32: SoC correction for HEV with auxiliary load over UDDS cycle. 103

Table 33: SoC correction for HEV w/o auxiliary load over UDDS1 cycle 105

Table 34: SoC correction for HEV with auxiliary load over UDDS1 cycle 107

Table 35: SoC correction for HEV w/o auxiliary load over UDDS2 cycle 109

Table 36: SoC correction for HEV with auxiliary load over UDDS2 cycle. 111 


\section{Nomenclature}

A

a

ADVISOR

APU

$\mathrm{C}_{\mathrm{b}}$

CBD

$\mathrm{C}_{\mathrm{D}}$ or $\mathrm{C}_{\mathrm{d}}$

CI

CSHVC

C1

C2

$\mathrm{CO}$

$\mathrm{CO}_{2}$

CVT

D

DR

di

$\mathrm{d}_{\mathrm{f}}$

$\mathrm{E}_{\mathrm{b}}$

$\mathrm{E}_{\mathrm{bi}}$

E.V
-Frontal Area in $\mathrm{m}^{2}$

-Acceleration in $\mathrm{msec}^{-2}$

-ADvanced VehIcle SimulatOR

-Auxiliary Power Unit

-Battery Energy Capacity in Ahr

-Central Business District

-Aerodynamic Drag Coefficient

-Compression Ignition

-City-Suburban Heavy Vehicle Cycle

-Constant

-Constant in $\sec ^{-1}$

-Carbon Monoxide

-Carbon Dioxide

-Continuously Variable Transmission

-Distance in $\mathrm{m}$

-Drive Ratio

-Tire Diameter

-Density of fuel

-Battery Energy in Joules

-Battery Energy at a given time step

-Electric Vehicle 


\begin{tabular}{|c|c|}
\hline EPA & -Environmental Protection Agency \\
\hline $\mathrm{F}$ & -Force in Newton \\
\hline $\mathrm{F}_{\mathrm{i}}$ & -Inertial Force \\
\hline $\mathrm{F}_{\mathrm{D}}$ & -Aerodynamic Drag Force \\
\hline $\mathrm{F}_{\mathrm{rr}}$ & -Rolling Resistance Force \\
\hline $\mathrm{F}_{\mathrm{w}}$ & -Force at the Wheels \\
\hline $\mathrm{F}_{\theta}$ & -Force Due to Road Grade \\
\hline GR & -Gear Ratio \\
\hline g & -Gravitational Acceleration in $\mathrm{msec}^{-2}$ \\
\hline $\mathrm{HEV}$ & -Hybrid Electric Vehicle \\
\hline $\mathrm{HC}$ & -Hydro Carbon \\
\hline ICE & -Internal Combustion Engine \\
\hline $\mathrm{I}_{\mathrm{b}}$ & -Battery Current \\
\hline $\mathrm{I}_{\mathrm{bmax}}$ & -Maximum Current out of Battery \\
\hline $\mathrm{I}_{\mathrm{bins}}$ & -Current out of Battery at a given Instant of Time \\
\hline $\mathrm{m}$ & -Mass in $\mathrm{kg}$ \\
\hline $\mathrm{m}_{\mathrm{f}}$ & -Mass Flowrate \\
\hline$\mu$ & -Rolling Resistance Constant \\
\hline$\eta_{\mathrm{dr}}$ & -Driveline Efficiency \\
\hline$\eta_{\text {tr }}$ & -Transmission Efficiency \\
\hline$\eta_{\mathrm{b}}$ & -Battery Efficiency \\
\hline $\mathrm{N}$ & -Speed in rpm \\
\hline $\mathrm{N}_{\mathrm{b}}$ & -Number of Batteries \\
\hline
\end{tabular}




\begin{tabular}{|c|c|}
\hline NMHC & -Non-Methane Hydrocarbons \\
\hline NOx & -Nitrogen Oxides \\
\hline NREL & -National Renewable Energy Laboratory \\
\hline NYC MTA & -New York City Metropolitan Transit Authority \\
\hline $\mathrm{P}$ & -Power in $\mathrm{kW}$ \\
\hline $\mathrm{P}_{\mathrm{bi}}$ & -Battery Power at a given time-step in $\mathrm{kW}$ \\
\hline $\mathrm{P}_{\mathrm{e}}$ & -Engine Power \\
\hline $\mathrm{PC}$ & -Planetary Combination \\
\hline $\mathrm{PM}$ & -Particulate Matter \\
\hline PNGV & -Partnership for a New Generation of Vehicles \\
\hline $\mathrm{P}_{\mathrm{w}}$ & -Power at the Wheels \\
\hline $\mathrm{P}_{\text {aux }}$ & -Auxiliary Load Power \\
\hline $\mathrm{P}_{\mathrm{r}}$ & -Power required in $\mathrm{kW}$ \\
\hline $\mathrm{P}_{\mathrm{m}}$ & -Motor Power \\
\hline$\rho$ & -Air Density \\
\hline SoC & -State of Charge \\
\hline $\mathrm{SoC}_{\mathrm{i}}$ & -State of Charge at a given time-step \\
\hline $\mathrm{SoC}_{\mathrm{t}}$ & -Target State of Charge \\
\hline TEOM & -Tapered Element Oscillating Micro Balance \\
\hline$\theta$ & -Road Grade Angle \\
\hline UDDS & -Urban Dynamometer Driving Schedule \\
\hline $\mathrm{V}$ & -Velocity in $\mathrm{msec}^{-1}$ \\
\hline $\mathrm{V}_{\mathrm{b}}$ & -Nominal Battery Voltage \\
\hline
\end{tabular}


WVU THDVETL -West Virginia University Transportable Heavy-Duty Vehicle

Emissions Testing Laboratories 


\section{SIMULATION OF HEAVY-DUTY HYBRID ELECTRIC VEHICLES}

\section{Introduction and Objectives}

The diesel engine is the most efficient power plant among all known types of internal combustion engines. Heavy trucks, urban buses, and industrial equipment are powered almost exclusively by diesel engines all over the world. In Europe, diesel powered cars have been increasingly popular. The diesel engine is a major candidate to become the power plant of the future. Before that happens, however, further progress in diesel emission control is needed.

The ever-depleting natural resources of oil have made people to think of the alternative ways of transportation. Global climate change has become an issue of primary concern. Existing technologies have improved the fuel economy and decreased tailpipe emissions of vehicles far beyond levels from only a decade or two ago, but further advances are both desired and required. Concern over these issues led the government and the automotive industry to form Partnership for a New Generation of Vehicles (PNGV). PNGV has a goal of tripling automobile fuel economy [1]. The $21^{\text {st }}$ Century Truck Program is a partnership between the heavy-duty truck and bus industry and the federal government for the development of technology. This technology will significantly reduce their emissions and use of fuel, doubling the fuel economy of Class 8 trucks and tripling the fuel economy of Class 2B and Class 6 trucks by 2010 as well as decreasing emissions and increasing safety [2].

Hybrid electric vehicles have gained attention due to the increased concern about the fuel economy and emissions from the vehicles. Though hybrids do not completely rule out the usage of crude oil, they decrease its usage to a considerable extent. Hybrid 
electric vehicles (HEVs) have electric energy storage systems such as batteries, ultra capacitors, or flywheels and power units such as an internal combustion engine (ICE) or a fuel cell. These vehicles show great potential for use in a wide variety of driving situations, but the optimization of components and control strategies is quite complex. Where as in a conventional vehicle an internal combustion engine drives a transmission that drives the differential, which in turn drives the wheels. Fuel economies of each modeled vehicle can be assessed in the following steps:

1) Hybrid optimization (step for electrification and engine optimization)

2) Engine downsizing (step for smaller and more advanced engine) and transmission switching if applicable

3) Load reduction (step for air/tire drag and weight reduction)

4) Dieselization (step for PNGV vehicles) [3].

With the introduction of fuel injection and catalyst aftertreatment, light-duty gasoline engines are both efficient (not as efficient as diesel engines) and maintain very low emission levels. But despite this clean engine management technology, fuel economy remains an issue due to the increased production of trucks and sport utility vehicles, which have much lower fuel economy due to poor aerodynamics, increased vehicle weight and large engine displacements. Many automobile manufacturers have embraced hybrid-electric drive as the next evolutionary step because the recovery of regenerative braking energy can largely offset the losses associated with greater vehicle weight. Generally speaking, a Hybrid Electric Vehicle (HEV) is defined as carrying at least two sources of motive energy on board and using electric drive to provide partial or complete drive power to the vehicle's wheels. One source is an electric motor supported by some 
type of energy storage device such as batteries, ultra capacitors, or flywheels. The other source has ranged from an internal combustion engine (ICE), to a gas turbine, or fuel cell. While ultra capacitors, flywheels, and fuel cells hold promise for the future, they have not reached a level of availability, reliability, and cost that would allow them to go into mass production in the near future. Further HEVs are classified under different categories. They are series, parallel, series-parallel and planetary HEVs. In this thesis, the HEVs simulated were assumed to be powered by a conventional diesel ICE, electric motor(s), and batteries. HEVs have several advantages over conventional vehicles. Hybrid-electric technology demonstrates a measurable advantage in city driving situations, when operated on stop-and-go, low-speed service applications. In this environment, regenerative braking can be utilized to recover kinetic energy normally lost to heat during mechanical braking [4]. On a conventional bus layout, the accelerator pedal controls the fuel delivery rate to the engine so when the pedal is depressed, more fuel is delivered to the engine. In a hybrid-electric vehicle, the accelerator pedal signals the vehicle computer, which in turn determines what amount of power is delivered by the battery and whether any additional power is required of the engine to either provide motive power or battery charging power.

In an HEV, the electric motor is used to decelerate the vehicle thereby generating power, which is stored in batteries and used to accelerate the vehicle. In a hybrid-electric vehicle the batteries (load-leveling device) receive energy via two mechanisms, regenerative braking and the auxiliary power unit (APU) generator. Since the engine reacts not only to the acceleration loads, but also to the battery State of Charge (SoC), the engine is not necessarily load following. In this thesis the vehicle considered for 
simulations is a transit bus. If a bus experiences a particularly difficult acceleration up a steep incline, energy from the batteries can be borrowed. The batteries may be sufficiently depleted to require recharging during cruise. If this occurs, the engine ramps up to generate excess energy that is provided to the batteries to maintain their SOC within a specified range. Over the course of a day's operation the battery SOC may fluctuate up and down several times. HEVs also allow downsizing of the ICE in most vehicles. The ICE in a conventional vehicle is sized to provide the peak power necessary to provide dynamic performance that is acceptable to the consumer. This peak power is seldom used and the engine often operates at low load and poor efficiency. In an HEV, peak power is provided by supplementing the ICE power with electric power allowing average engine operation to be closer to the optimum range increasing efficiency and often decreasing the weight of the vehicle.

Objectives of this thesis were to develop simulation models for a series hybrid electric vehicle (transit bus) using excel spreadsheets and ADVISOR package. Validating these simulations by comparing fuel economy data thus obtained with those of in-use vehicles. Also predicting $\mathrm{NOx}$ based on $\mathrm{CO}_{2}$ emissions was one of the objectives as axle power is not due to engine power alone in HEVs.

In this thesis a certain control strategy was developed in spreadsheets for a series hybrid electric vehicle. Simulations were run in the spreadsheet model and in a computerbased vehicle simulation model, ADVISOR, to predict fuel economy of both conventional and hybrid electric heavy-duty vehicles. Actual data collected from chassis tests of conventional and hybrid electric vehicles were used to validate the predicted fuel economy. The fuel consumed was modeled since it provides a good indication of engine 
operation, and also NOx emissions were modeled in this thesis since it is of primary concern for compression ignition (CI) engines. The development of more accurate continuous emissions models will enhance vehicle design optimization, especially for hybrid vehicles, as well as improve the prediction capabilities of emissions inventory through vehicle runs and Monte-Carlo simulations.

\subsection{Literature Review}

The idea of electric and hybrid electric vehicles is not new. The first vehicle powerplants were steam engines, internal combustion engines, and electric motors. Professor Stratingh in the Dutch town of Groningen made the first electric vehicle (EV) in 1835 . But the first practical electric road vehicle was probably made either by Thomas Davenport in the United States or by Robert Davidson in Edinburgh, Scotland in 1842. These pioneers had to use non-rechargeable electric cells. An electric vehicle did not become a viable option until the Frenchmen Gaston Plante and Camille Faure respectively invented (1865) and improved (1881) the storage battery. Although several electric vehicle manufacturers were established in Europe as well as in America before internal combustion engines became available, the electric vehicle did not become a viable option until the storage battery was invented and improved. At the turn of the century (1899), Baker Electric, in the USA, manufactured an electric vehicle that was reputedly easy to drive, could cruise a distance of 80 kilometers when fully charged and was capable of reaching a top speed of $40 \mathrm{~km} / \mathrm{h}$. The vehicle had rechargeable batteries as an energy storage device. The two technologies were combined to provide the increased range of an ICE powered vehicle with the safety and reliability of an electric vehicle. 
Increasingly powerful and reliable ICEs eventually replaced the widespread use of electric motors [5].

Recent years have seen growth in the popularity of HEVs both in light and heavyduty applications. Examples of current production HEVs include light-duty vehicles such as the Toyota Prius and Honda Insight, and heavy-duty vehicles such as Orion/LockheedMartin transit buses. These vehicles have demonstrated the advantages of HEVs to the public and they can only be expected to become more popular.

The most common current use for heavy-duty HEVs is in the transit bus industry. In-use data from fleets in New York City have demonstrated $30-50 \%$ gains in fuel economy as well as $30-60 \%$ lower $\mathrm{NO}_{\mathrm{x}}$ and $\mathrm{HC}$, and $20-40 \%$ lower greenhouse gases. Also with the use of trap filters, 50 - 90\% lower PM emissions were obtained [7].

\subsection{ADVISOR}

ADVISOR is a vehicle simulator designed to perform computer simulation on conventional, electric, and hybrid electric vehicles. The ADVISOR (ADvanced VehIcle SimulatOR) software package was developed at National Renewable Energy Laboratory (NREL) and is available from their web site [8]. ADVISOR simulates the behavior and response of the vehicle's components on a driving cycle. The driving cycle is a speed and

road grade versus a time trace. The simulation uses component models to predict performance, fuel efficiency, and emissions for a vehicle.

ADVISOR uses models for engines, transmissions, electric motors, and fuel cells. For each model, a torque and speed are requested, and the speed and torque achieved are passed to the next model. These models contain the component efficiency. The efficiency 
is constant for simple components and lookup tables are used for more complex components such as the engine and electric motor. ADVISOR also includes neural network based energy storage system models for various types of battery packs. The models determine the efficiency based upon the load on the component. Accessory loads to the engine can also be modeled by ADVISOR, which allows the option of choosing the application for the accessory load that varies with vehicle size. The fuel converter models in ADVISOR include emissions maps on certain engine models. These include hydrocarbons $(\mathrm{HC})$, carbon monoxide $(\mathrm{CO})$, particulate matter $(\mathrm{PM})$, and oxides of nitrogen (NOx). The emissions data were based upon engine dynamometer tests with the data interpolated linearly to fit the percent torque at a given speed [9].

\subsection{Emissions Testing}

The emissions data used in this thesis were collected from one of the West Virginia University Transportable Heavy Duty Vehicle Emissions Testing Laboratories. Each laboratory consists of a mobile chassis test bed and emission analyzer trailer. The test vehicle is driven onto the test bed via ramps and chained down with the drive wheels supported via four pairs of idle rollers. Right and left sets of flywheel weights and eddy current power absorbers load the vehicle via drive shafts connected to right and left hubs of the vehicle. Two Mustang air-cooled eddy current power absorbers each rated at 224 $\mathrm{kW}$ continuous load, and $745 \mathrm{~kW}$ peak load simulate Road load (wind and rolling resistance). Axle torque is measured with two Eaton torque transducers (one for each axle) with a $22,600 \mathrm{Nm}\left(16,669 \mathrm{ft}-\mathrm{lb}_{\mathrm{f}}\right)$ rating. Vehicle exhaust is ducted to a full flow dilution tunnel with flow rate controlled by a critical flow venturi. Sample probes near 
the end of the dilution tunnel deliver diluted exhaust gas samples to the analyzer bench for continuous concentration measurement of $\mathrm{HC}, \mathrm{CO}, \mathrm{CO}_{2}$ and $\mathrm{NO}_{\mathrm{x}}$ [10]. Chassis dynamometer testing is more representative of actual in-use vehicle operation as it accounts for the losses and operation associated with the specific vehicle into which the engine is installed. Chassis testing can also accurately measure the system benefits of hybrids including the recovery of braking energy through regenerative braking, greater driveline efficiency and reduced transient operation of the engine powering the auxiliary power unit.

In this thesis the simulation models were developed in Excel Spreadsheets. The results of which were compared with those of simulations run in ADVISOR. Further the fuel economy results obtained from these simulation models were compared with those of the on-road vehicles.

\subsection{Health Concerns}

Internal combustion engines are significant contributors to air pollution, which has a damaging impact on our health and the environment and is suspected to cause global climate changes. The specific problem posed by heavy-duty vehicles is that they remain in service for many more years than passenger cars. The high proportion of older trucks and buses on the road causes most of the pollution, making it desirable to limit pollution from vehicles in service as well as new units Environmental benefits of diesels, such as low greenhouse gas emissions, are balanced by growing concerns with emission of oxides of nitrogen and diesel particulate. Emissions from diesel engines contribute to serious public health problems in the U.S. These problems include premature mortality, 
aggravation of respiratory and cardiovascular disease, aggravation of existing asthma, acute respiratory symptoms, chronic bronchitis, and decreased lung function. Numerous studies also link diesel exhaust to increased incidence of lung cancer. On-road dieselfueled vehicles contribute approximately 27 percent of the NOx emission inventory and 62.5 percent of the PM emissions. An older, dirtier diesel vehicle can emit almost 8 tons of pollution per year. This amounts to 160 to 240 tons of pollution over the life of the engine. A heavy-duty truck can create the same amount of air pollution as 150 passenger cars [11].

Increasingly tighter environmental regulations worldwide call for advanced emission controls and near-zero diesel emission levels in the years to come. During the 1990s, the EPA recognized that engines and fuels needed to be controlled together to achieve the lowest emissions from heavy-duty vehicles [12]. Table 1 shows the EPA standards for heavy-duty diesel engines. Table 2 shows the California emission standards for heavy-duty diesel engines. 


\begin{tabular}{|l|l|l|l|l|}
\hline Year & HC & CO & NOx & PM \\
\hline Heavy-Duty Diesel Truck Engines \\
\hline 1990 & 1.3 & 15.5 & 6 & 0.6 \\
\hline 1991 & 1.3 & 15.5 & 5 & 0.25 \\
\hline 1994 & 1.3 & 15.5 & 5 & 0.1 \\
\hline 1998 & 1.3 & 15.5 & 4 & 0.1 \\
\hline Urban Bus Engines \\
\hline 1991 & 1.3 & 15.5 & 5 & 0.25 \\
\hline 1993 & 1.3 & 15.5 & 5 & 0.1 \\
\hline 1994 & 1.3 & 15.5 & 5 & 0.07 \\
\hline 1996 & 1.3 & 15.5 & 5 & 0.07 \\
\hline 1998 & 1.3 & 15.5 & 4 & 0.07 \\
\hline
\end{tabular}

Table 1: EPA Emissions Standards for Heavy-Duty Diesel Engines, g/bhp·hr [12]

\begin{tabular}{|c|c|c|c|c|c|}
\hline Year & NMHC & $\mathrm{HC}$ & $\mathrm{CO}$ & NOx & $\mathrm{PM}$ \\
\hline \multicolumn{6}{|c|}{ Heavy-Duty Diesel Truck Engines } \\
\hline 1987 & & 1.3 & 15.5 & 6 & 0.6 \\
\hline 1991 & 1.2 & 1.3 & 15.5 & 5 & 0.25 \\
\hline 1994 & 1.2 & 1.3 & 15.5 & 5 & 0.1 \\
\hline \multicolumn{6}{|c|}{ Urban Bus Engines } \\
\hline 1991 & 1.2 & 1.3 & 15.5 & 5 & 0.1 \\
\hline 1994 & 1.2 & 1.3 & 15.5 & 5 & 0.07 \\
\hline 1996 & 1.2 & 1.3 & 15.5 & 4 & 0.05 \\
\hline
\end{tabular}

Table 2: California Emissions Standards for Heavy-Duty Diesel Engines, g/bhp·hr [12]

On January 18, 2001, the EPA adopted even lower emission standards for heavyduty diesel engines. These standards aim to reduce those engines' emissions by $95 \%$ from current levels. The latest standards will reduce NMHC emissions to $0.14 \mathrm{~g} / \mathrm{bhp}-\mathrm{hr}$ and NOx emissions to $0.20 \mathrm{~g} / \mathrm{bhp}-\mathrm{hr}$; both these emission standards will be phased in on a percent-of-sales basis between 2007 and 2010. The new PM emission standard of 0.01 $\mathrm{g} / \mathrm{bhp}-\mathrm{hr}$ will take full effect in 2007 . The effect of the latest standards is equivalent to eliminating the pollution produced by 13 million of today's trucks. 
The EPA has pursued reduction in diesel fuel sulfur levels in two steps in the 1990s. In 1993, the EPA adopted regulations that reduced diesel sulfur from an average 2,500 parts per million (ppm) to $500 \mathrm{PPM}$, an $80 \%$ reduction. The latest round of EPA regulations will reduce diesel sulfur levels to 15 PPM - a 97\% reduction from current levels -- beginning June 1, 2006.

It is estimated that the latest regulation will increase the cost of a new truck by $\$ 1,200-\$ 1,900$, depending on vehicle size, compared with new heavy-duty trucks, which can cost $\$ 150,000-\$ 250,000$. The agency also estimates that reducing the sulfur cap to 15 PPM will add about $\$ 0.045-\$ 0.05$ per gallon to the current price of diesel fuel. Heavyduty engines can operate for up to 30 years or 1.5 million miles. At an average fuel economy of 5 miles per gallon of diesel fuel, over its service life, a heavy-duty engine would use 300,000 gallons of diesel, resulting in an additional cost of $\$ 13,500-\$ 15,000$ over its lifetime, or about $\$ 450-\$ 500$ more per year [13]. 


\section{Types of Vehicle Layouts}

Different types of vehicle configurations are conventional, electrical and hybrid electrical vehicles. Further, hybrid electric vehicles are subdivided into three types. They are Series HEV, Parallel HEV and Combination HEV. This Combination HEVs are again branched into Series-Parallel Combination HEV and Planetary Combination HEV.

\subsection{Conventional Vehicle}

In a conventional vehicle an internal combustion engine drives a transmission that drives a differential, which in turn drives the wheels. The engine can be fueled by diesel or gasoline. The transmission can be manual, automatic or continuously variable (CVT). A conventional vehicle is relatively inexpensive and easy to control. It does not require extra control besides the engine control unit and the automatic transmission control unit if an automatic transmission is applied. The main disadvantages are lack of regenerative braking and tailpipe emissions. Figure 1 shows the layout of a conventional vehicle. 


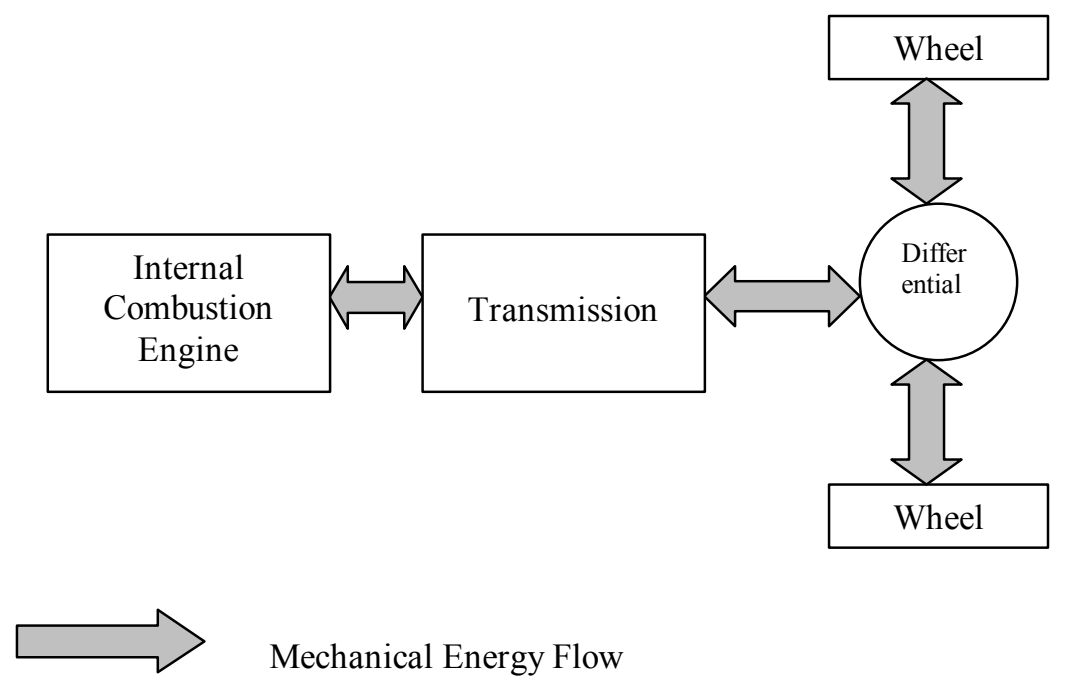

Figure 1: Layout of Conventional Vehicle [6]

\subsection{Electric Vehicle}

An electric vehicle has an energy storage device called a battery that supplies electric energy to the motor, which powers the wheels of the vehicle. The advantages of electric vehicles include zero tailpipe emissions and noiseless operation compared to most conventional vehicles. Disadvantages are due to short range and long recharge times. Current production EVs are limited to a maximum of approximately 257 kilometers on one battery charge without the use of air conditioning [6]. This is sufficient for many daily commuters but not for long trips. In addition to this short range, the batteries require several hours to recharge once depleted. The short range of electric vehicles is not the main problem though. While conventional vehicles can be refilled in a couple of minutes, batteries of EVs need several hours of charging once they were discharged. The idea of not being able to use a vehicle for several hours out of every day is unacceptable to many consumers. Electric vehicle layout is shown in the Figure 2. 


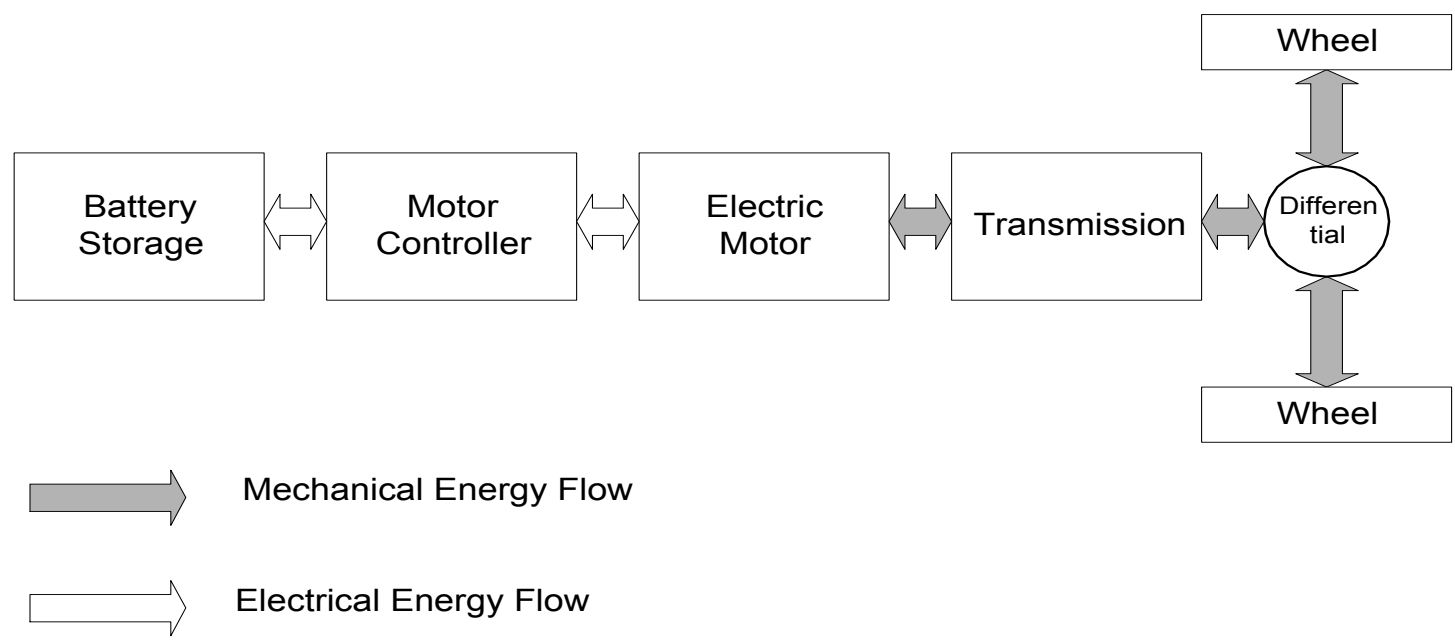

Figure 2: Layout of Electric Vehicle [6]

\subsection{Series Hybrid Electric Vehicle}
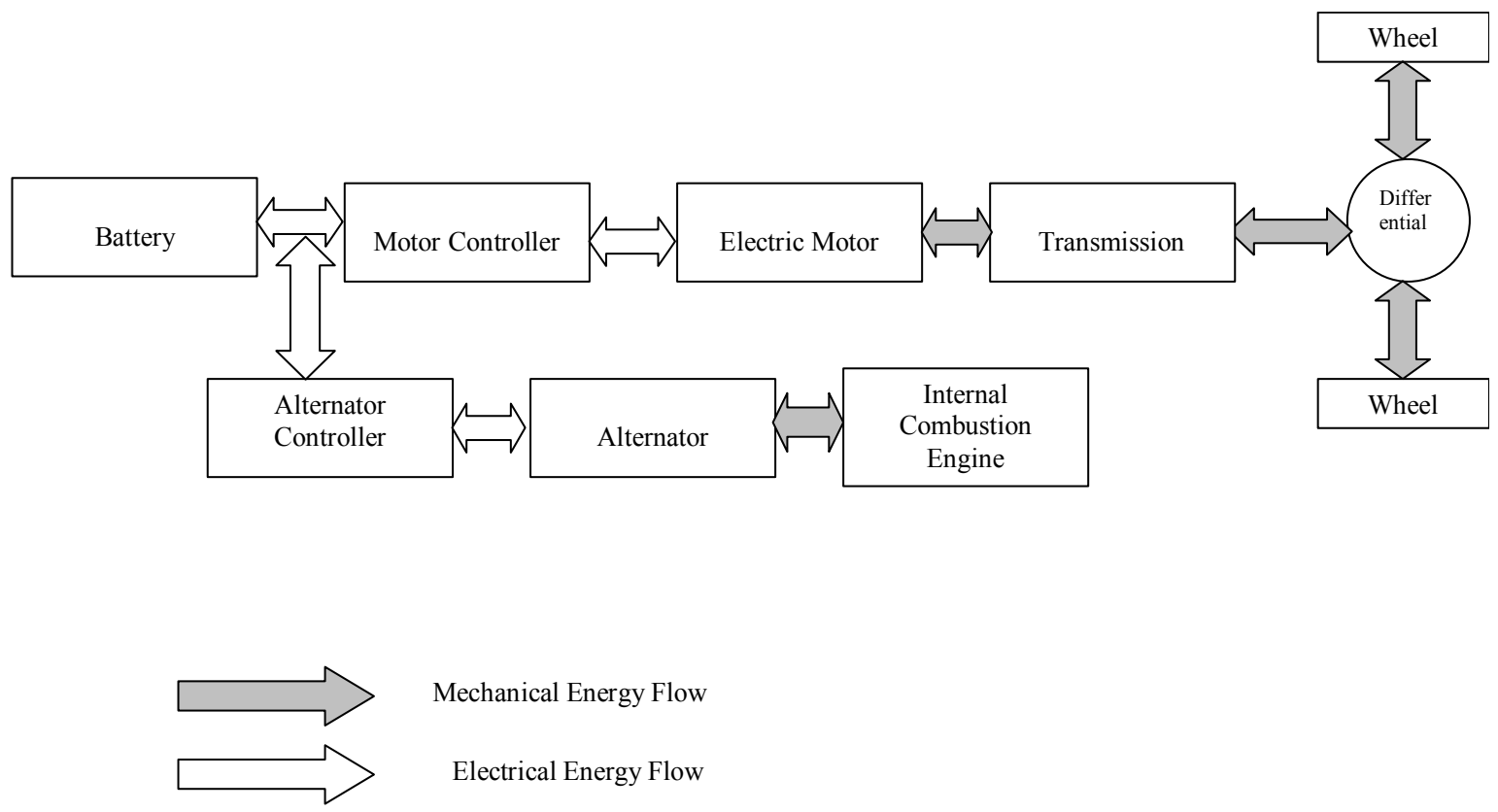

Figure 3: Layout of Series HEV with power flowing into the ICE while coasting down the hill, engine acting as pump 
Figure 3 shows a typical series HEV layout. In a series HEV, an ICE or fuel cell is used to produce electrical energy that is sent to the battery pack and electric motor. Electric motor supplies all of the power required to drive the vehicle. In series HEVs there is no physical coupling between the engine and the transaxle. This can reduce the transient operation of the ICE that is especially helpful from an emissions standpoint allowing optimal fueling and ignition control. Disadvantages in current series HEVs include losses during changing energy from chemical to mechanical, mechanical to electrical, and electrical to mechanical forms and the need for costly, heavy battery packs and electric motors. Series vehicles typically show substantial fuel economy improvements in highly transient driving in urban situations due to recovery of large amounts of regenerative braking energy. Smaller efficiency gains are realized through less transient operation such as highway driving, where there is less available regenerative braking energy.

\subsection{Parallel Hybrid Electric Vehicle}

In a parallel HEV, there is a direct connection between both the ICE and the electric motor and the wheels as shown in Figure 4. This configuration allows a wide variety of control strategies to be employed. When high power is demanded such as for high acceleration, both the ICE and electric motor deliver power to the wheels. In less demanding situations, the ICE can be operated at a higher power, which is required to drive the vehicle and the excess power is stored in the batteries for later use, or the electric motor alone can be used to drive the vehicle. This has the advantage of operating the ICE in a more efficient mode or not at all. During long, steady state cruises, the ICE 
engine alone can drive the vehicle avoiding the inherent inefficiency of the batteries. The main advantage of parallel HEVs is improved dynamic performance due to the direct coupling between the ICE, electric motor, and the wheels. The disadvantage with the ICE being directly coupled to the wheels is that there is more transient speed operation than in a series vehicle. This tends to result in poorer efficiency and increased emissions.

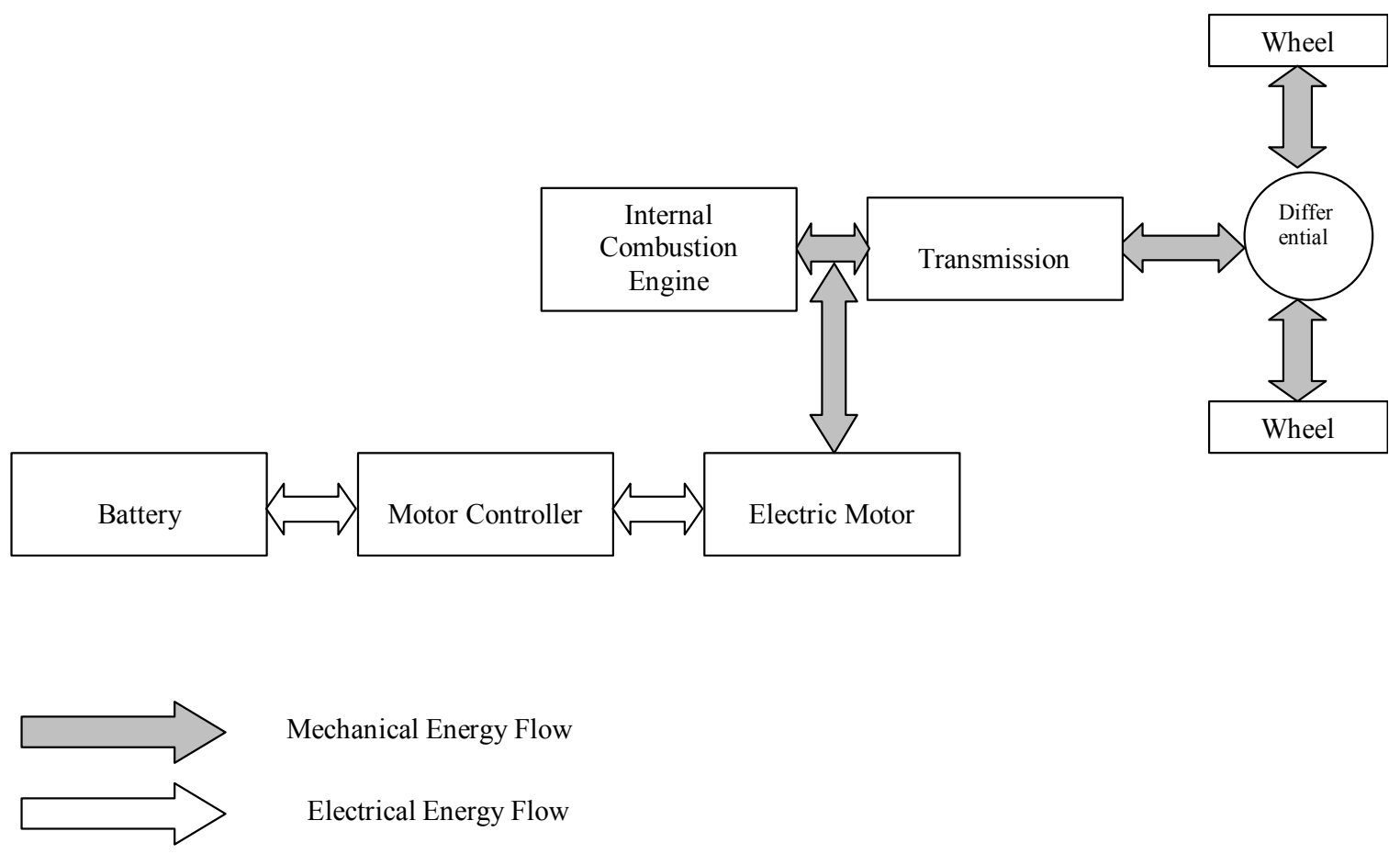

Figure 4: HEV with batteries and ICE connected in parallel

\subsection{Combination Hybrid Electric Vehicle}

There are several combinations for the drive train that have the characteristics of both a series HEV and a parallel HEV. The two main layouts are the series-parallel combination and the planetary combination (PC) HEVs. The series-parallel combination operates as a series HEV at one instance and as parallel HEV at another instance. The 
series-parallel combination has two electric motors and an ICE coupled with a combination of clutches. Also the control of these vehicles is more complex than series HEVs or parallel HEVs. Figure 5 shows the layout of a series-parallel HEV.

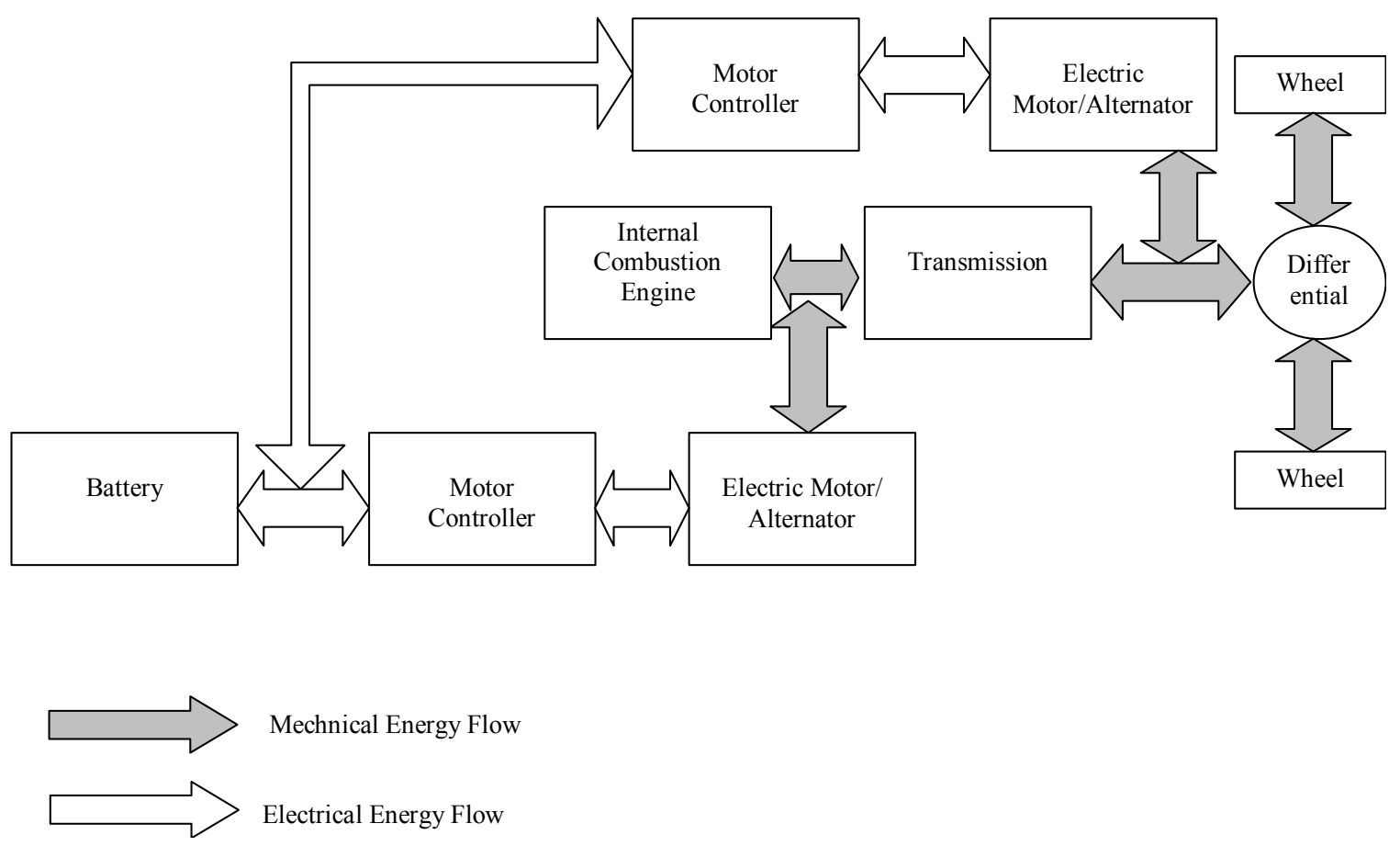

Figure 5: Layout for a Series-Parallel HEV [6]

The PC hybrid in the Toyota Prius couples an ICE, an alternator, and a motor via a planetary gear set. The engine is linked to the planet carrier; the alternator to the sun gear and the output is the ring that transmits the torque to the differential. The motor is also linked to the ring gear so that it is able to add torque to the output shaft and also to the differential. With this setup there are three degrees of freedom, with the alternator being used to control the extra degree of freedom on the sun. The engine operates at the most efficient point at each speed of operation as the alternator controls the torque. As there is no gear changing involved with the $\mathrm{PC}$ hybrid the engine operation is less transient than at the parallel configuration. It is not as steady as the series though. In this setup the vehicle acts as a series HEV, only when the stationary vehicle starts moving. As 
soon as the vehicle starts moving, besides the electrical path, power gets transmitted mechanically through the planetary gear set. For the rest of its operation the vehicle works as a combination of a series and a parallel HEV, by taking advantage of both configurations. If the alternator could be stopped the vehicle would operate as a parallel vehicle. The hardware cost of the PC hybrid is more than that of an electric or a conventional vehicle. It needs two electric motors and an engine but it also eliminates the need for the transmission that makes the PC hybrid one of the cheapest most integrated designs. The control of the PC hybrid is more complicated than that of the series and less complicated than that of the parallel HEV. Figure 6 shows the basic layout of the PC hybrid [6].

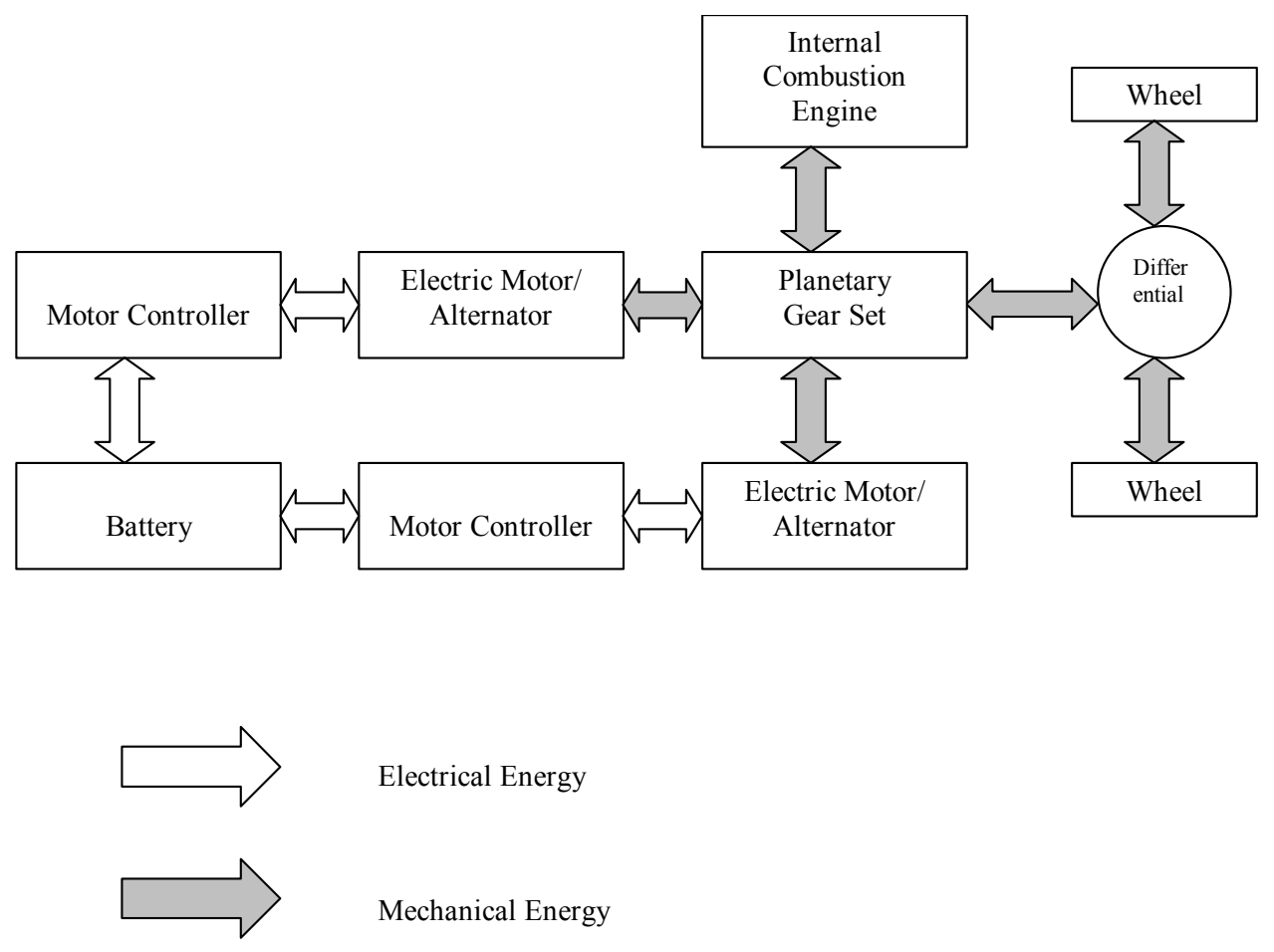

Figure 6: Layout for a Planetary Combination HEV [6] 


\section{Drive Cycles}

A driving cycle represents different modes of vehicle operation (speed and time trace). It provides the basis for evaluating the effects of those modes on fuel economy of the vehicle tested. Also, the drive cycle has a significant effect on measured emission levels. Various drive cycles with varying average speed and number of stops per mile to develop a more rounded comparison between the conventional buses and hybrid buses were chosen for simulation. The Central Business District (CBD) Cycle is a chassis dynamometer testing procedure for heavy-duty vehicles ( $S A E$ J1376). The CBD cycle represents a "sawtooth" driving pattern, which includes 14 repetitions of a basic cycle composed of idle, acceleration, cruise, and deceleration modes [14]. This cycle has an average speed of $12.71 \mathrm{mph}$ over a driving distance of 2 miles in 560 seconds. Vehicle speed over the duration of the CBD cycle is shown in Figure 7. 


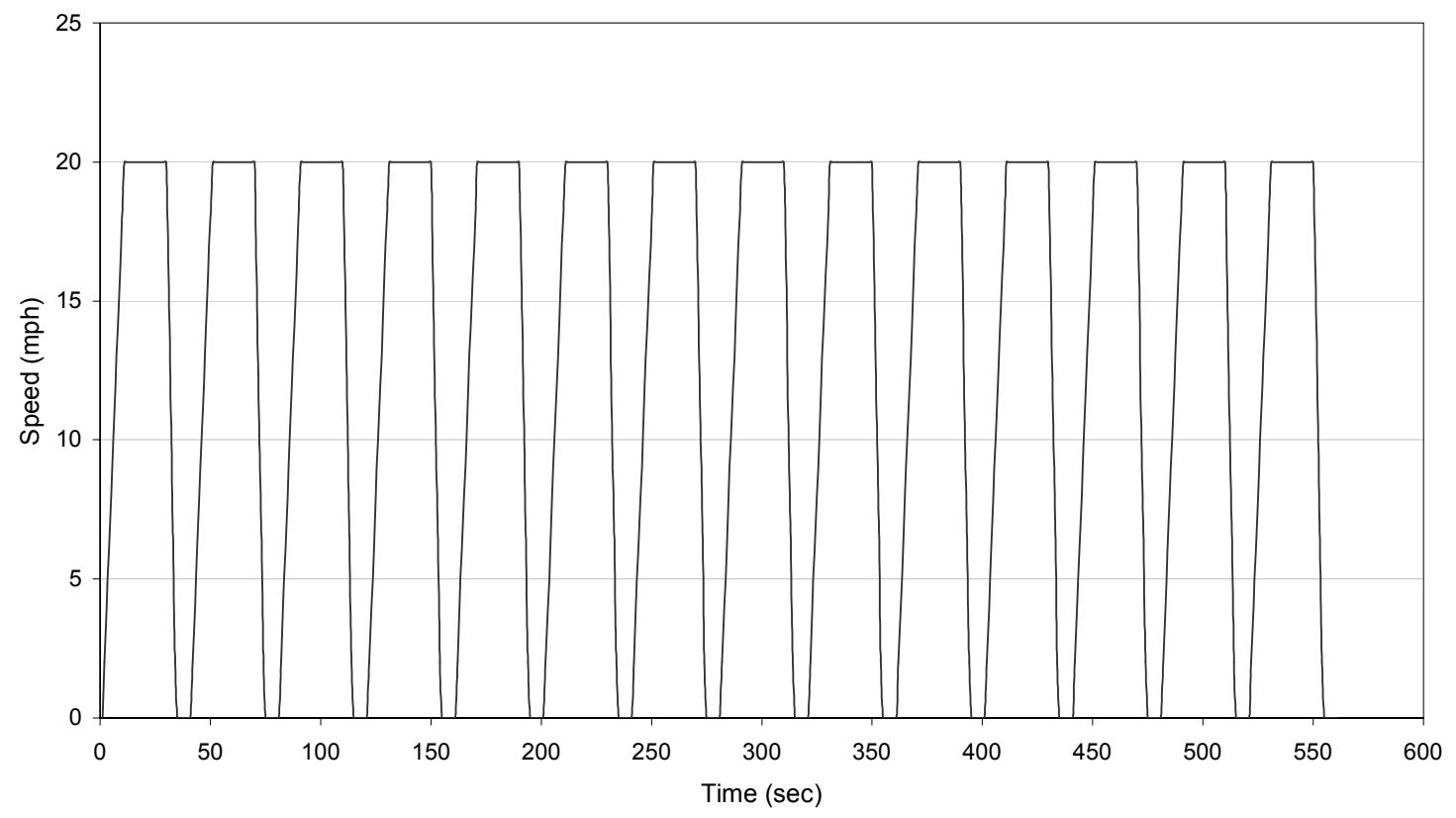

Figure 7: CBD cycle consists of 14 peaks and has a maximum speed of $20 \mathrm{mph}$.

Test D, also known as the 'EPA Urban Dynamometer Driving Schedule for Heavy Trucks' or UDDS, was developed using data logged from buses, trucks, and tractor-trailers operating in New York and Los Angeles under both freeway and nonfreeway conditions. A Monte Carlo simulation was then used to produce the cycle [14]. This cycle is run over a driving distance of 5.55 miles for 1060 seconds with a maximum speed of $58 \mathrm{mph}$. Vehicle speed over the duration of the UDDS cycle is shown in Figure 8. 


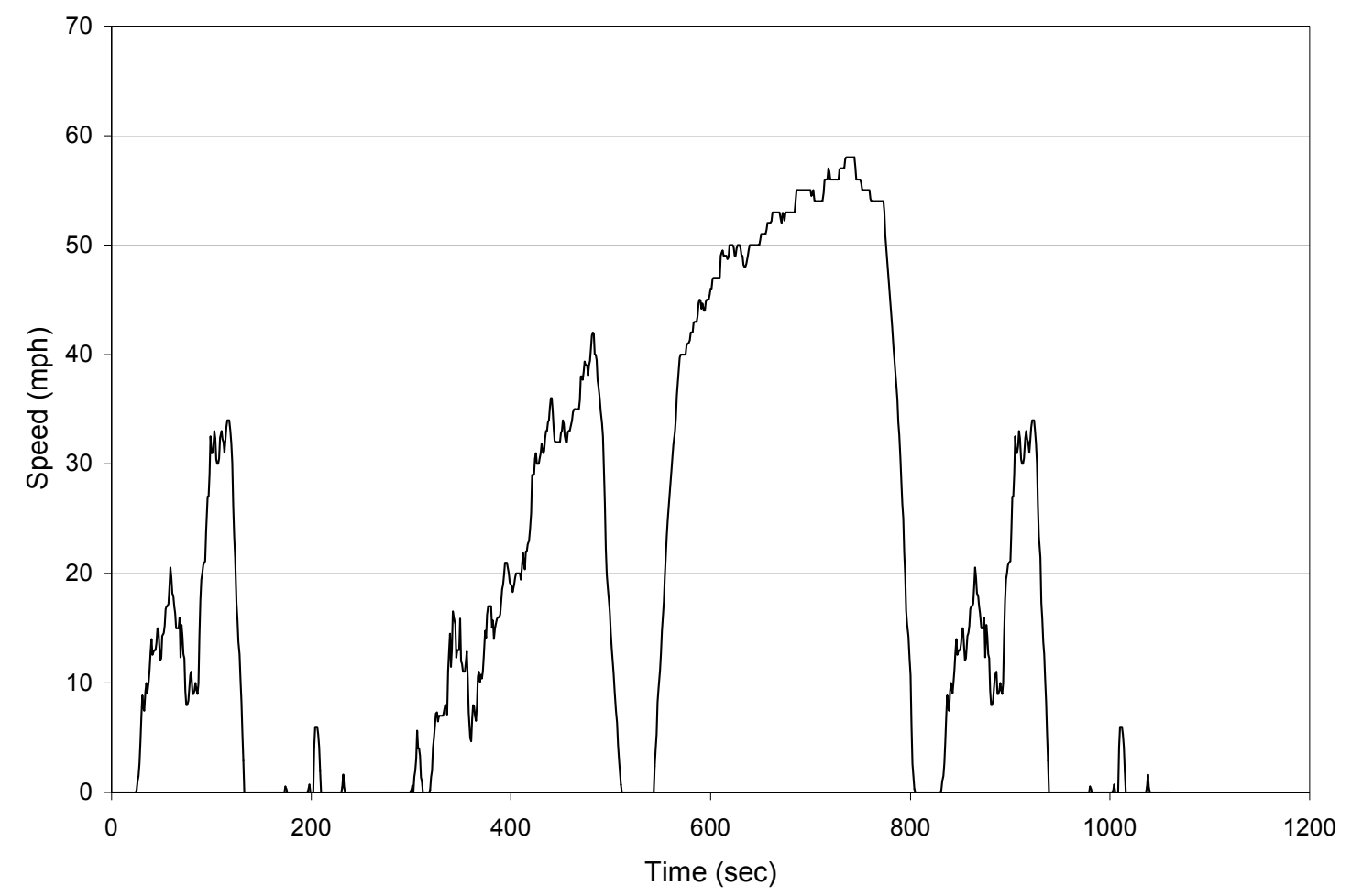

Figure 8: Speed vs Time trace for UDDS cycle with an average speed of $18.86 \mathrm{mph}$.

WVU developed a new cycle called Manhattan cycle utilizing actual in-use route segments data logged from New York City Metropolitan Transit Authority (NYC MTA) buses operating in Manhattan. The data collected was divided into micro-trips consisting of a start from idle, acceleration to speed, and deceleration back to idle. This cycle has 20 micro-trips covering 2.1 miles in 1083seconds with an average speed of $6.83 \mathrm{mph}$ [4]. Vehicle speed over the duration of the Manhattan cycle is shown in Figure 9. 


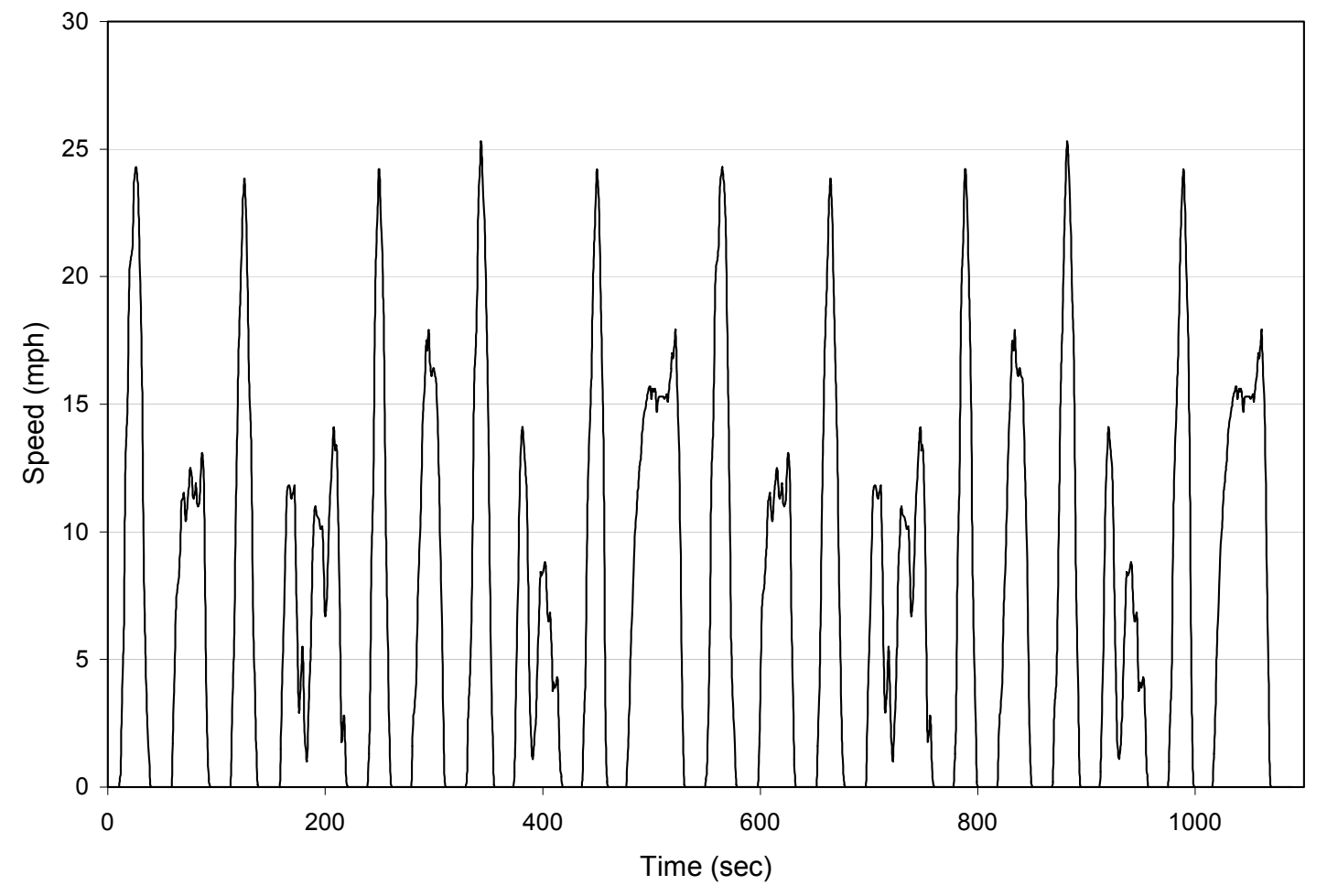

Figure 9: Manhattan cycle has 20 micro trips over a distance of 2.1 miles.

The City-Suburban Heavy Vehicle Cycle (CSHVC) is composed of data taken from trucks traveling in dense traffic with stoplights as well as delivery routes on the outskirts of cities [15]. The cycle is run over a driving distance of 6.68 miles in 1700 seconds with an average speed of $14.15 \mathrm{mph}$. Vehicle speed over the duration of the CSHVC is shown in Figure 10. 


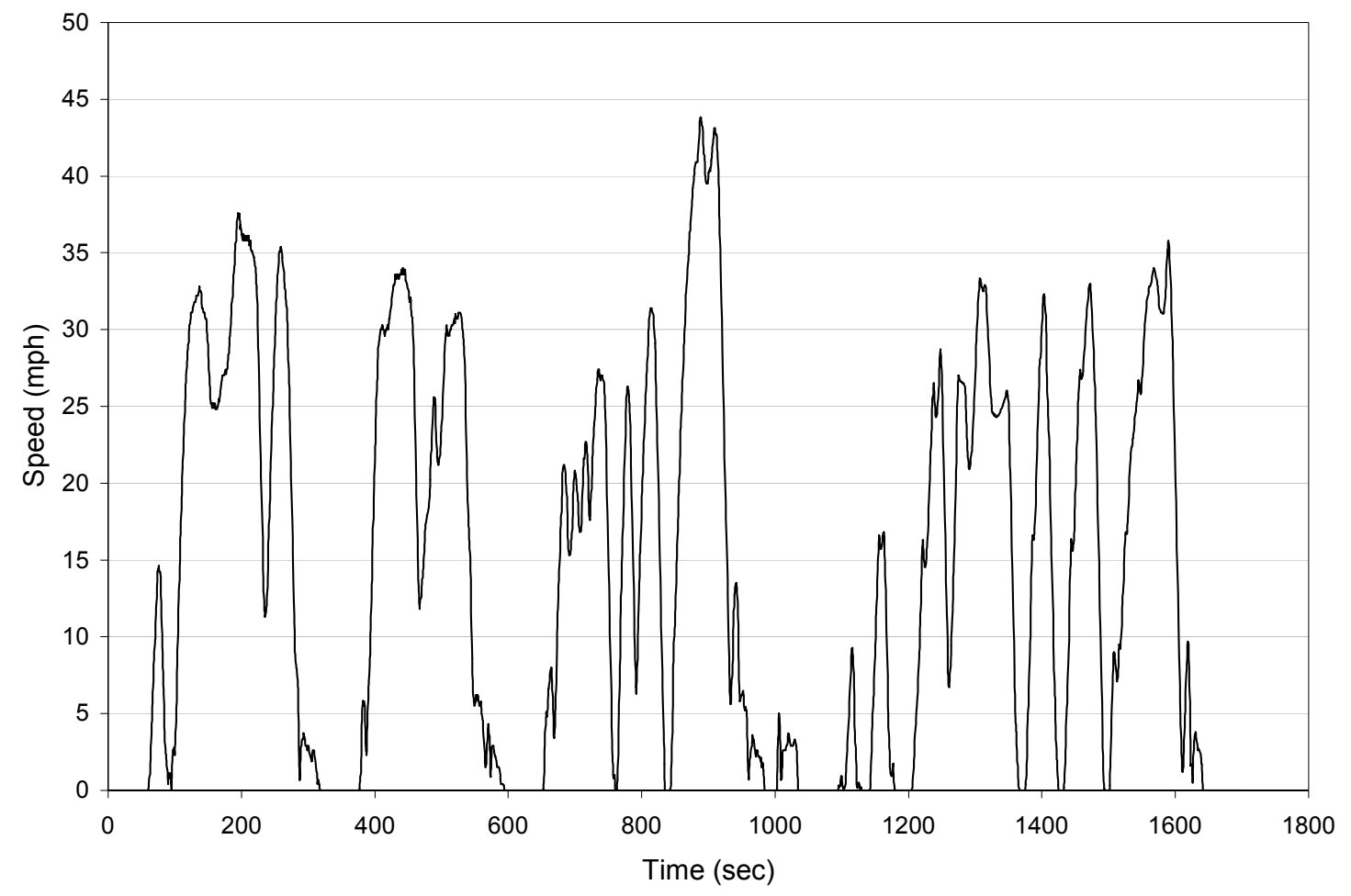

Figure 10: Speed Vs Time trace for CSHVC with a maximum speed of $43.8 \mathrm{mph}$.

The peaks of speed and time trace for UUDS cycle were rearranged in this thesis to study the effects of regenerative braking. Thus two new cycles were formed. They are referred to as UDDS1 cycle and UDDS2 cycle shown in Figure 11 and Figure 12 respectively. Maximum speed peaks were arranged at the end of the cycle in UDDSland at the beginning of the cycle in UDDS2. Generally vehicles stop faster than accelerate. In hybrid vehicles acceleration depends on drive system power and capture of regenerative braking power while decelerating depends on capacity of batteries. With the maximum speed peaks at the beginning of the cycle in UDDS2, the engine is driven hard. In UDDS1 the maximum speed peaks are at the end of the cycle making the batteries to deplete. 


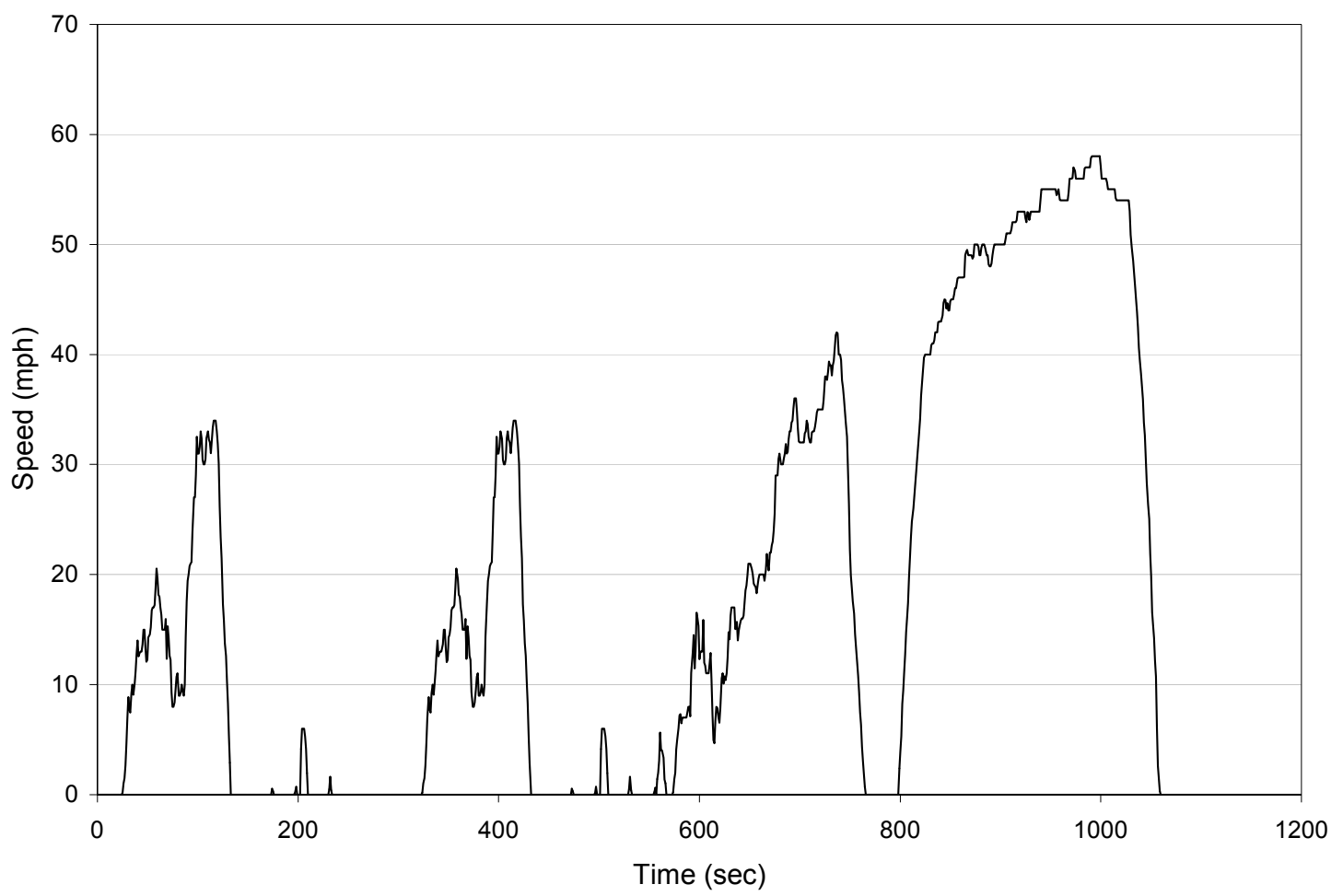

Figure 11: UDDS1 with highest peak at the end of cycle.

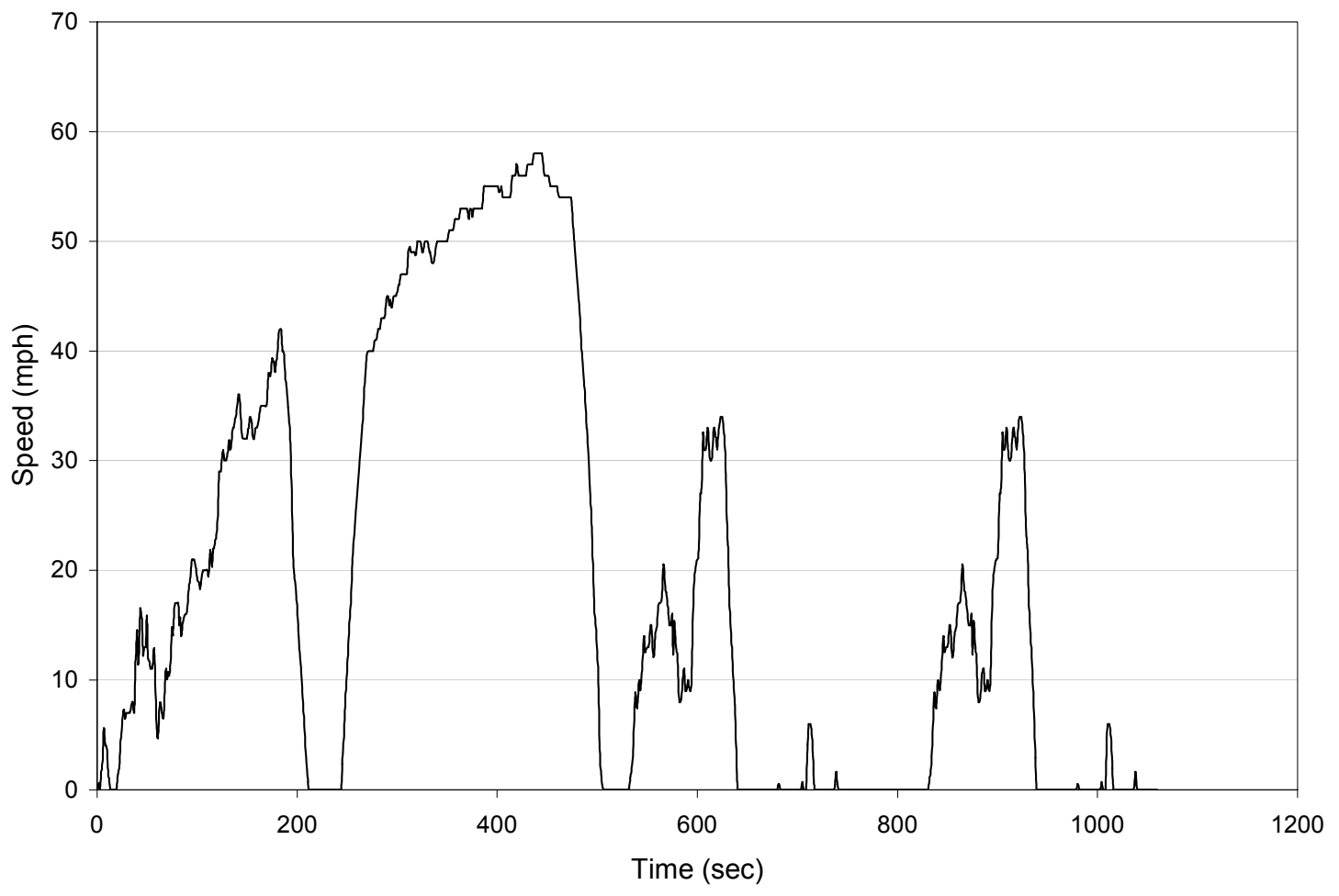

Figure 12: Speed vs Time trace for UDDS2 cycle 


\subsection{Power Consumed by a Vehicle}

Newton's second law of motion states that the summation of forces acting upon an object is equal to the object's mass multiplied by the acceleration of the object. The acceleration of a vehicle in motion depends on the force acting upon the vehicle and mass of the vehicle.

$$
\sum F=m a
$$

The forces acting on a moving vehicle include aerodynamic drag, rolling resistance, road grade force, and inertial force.

$$
\Sigma F=F_{i}=F_{w}-F_{D}-F_{r r}-F_{\theta}
$$

where $F_{i}$ is the inertial force,

$\mathrm{F}_{\mathrm{w}}$ is force at wheels,

$\mathrm{F}_{\mathrm{D}}$ is drag force,

$\mathrm{F}_{\mathrm{rr}}$ is rolling resistance and

$F_{\theta}$ is road grade force.

The aerodynamic drag on an object is based on the density of the fluid through which it is traveling, its speed, its drag coefficient, and its frontal area. It is the force required to push the vehicle through the air.

$$
F_{D}=1 / 2 \rho V^{2} C_{D} A
$$

where $\rho$ is density of air,

$\mathrm{V}$ is velocity of the vehicle,

$\mathrm{C}_{\mathrm{D}}$ is drag coefficient and

A is vehicle frontal area. 
Rolling resistance comes from a combination of the weight of the vehicle deforming the shape of the tire, the friction between the tire and the roadway, and air friction across the tire surface [16]

$$
F_{r r}=\mu m g \cos \theta
$$

where $\mu$ is the coefficient of rolling resistance,

$m$ is mass of the vehicle,

$\mathrm{g}$ is the acceleration due to gravity and

$\theta$ is angle of inclination or grade.

The force on a vehicle due to road grade is due to a portion of the vehicle's weight vector being directed against the direction of travel when $\theta$ is positive and with the direction of travel when $\theta$ is negative.

$$
F_{\theta}=m g \sin \theta
$$

Since power can be calculated from,

$$
P=F V
$$

Multiplying Equation 2 by the vehicle speed yields,

$$
m V \frac{d V}{d t}=F_{w} V-\left(1 / 2 \rho V^{3} C_{D} A+\mu m g V \cos \theta+m g V \sin \theta\right)
$$

where $\frac{d V}{d t}$ is rate of change of speed.

Finally, the power to move a vehicle is based on its aerodynamics, the rolling resistance of its tires, the road grade, the desired acceleration and velocity.

In addition to the power requirements for driving the vehicle, auxiliary loads and driveline efficiencies can make a significant difference in the power required from the engine. Here, the transmission is assumed to be $95 \%$ efficient under low loads. Accessory 
loads include the power needed to drive air conditioning systems, power steering, and power for the radiator fan and electrical loads. Accessory loads were assumed to be $15 \mathrm{~kW}$ for the heavy-duty vehicles. The load represents the load from the air conditioner and other accessories for a city bus with a full load of passengers [8]. The overall power required from the engine for a conventional vehicle is given by

$$
P_{e}=\frac{P_{w}}{\eta_{t r}}+P_{a u x}
$$

The significance of each of the power requirements to drive the vehicle changes under different conditions. In this thesis the vehicle is assumed to be driving on flat road with grade angle of $0^{\circ}$.

In Figures 13 through 18, the total energy requirements for the vehicle over the cycle can be found by the summation of positive and negative areas under the power trace. The negative portion of the power trace represents the opportunity to capture regenerative braking energy. In a conventional vehicle, this energy is lost through service braking. The consumer expectations of dynamic performance are quite low. Also, the power needed to meet the trace exactly would require a very powerful engine or engineelectric motor combination that would tend to be oversized and inefficient under less demanding situations.

Figures 13 through 18 show the power requirement of a heavy-duty vehicle over various driving cycles. The power demand shows that there are large spikes with considerable amount of regenerative power. Any hybrid system that relies heavily on battery power would be at a major disadvantage. Since there is little opportunity for regenerative braking, any use of the motor would deplete the batteries without a means to recharge them. This limits the use of the hybrid system. In the Figure 13, the peaks of 
power required vary in amplitude because of the non-uniform rate of acceleration and deceleration for a CBD cycle. When a vehicle is following a speed vs time trace, depending on the driver, there would be different rates of acceleration and deceleration.

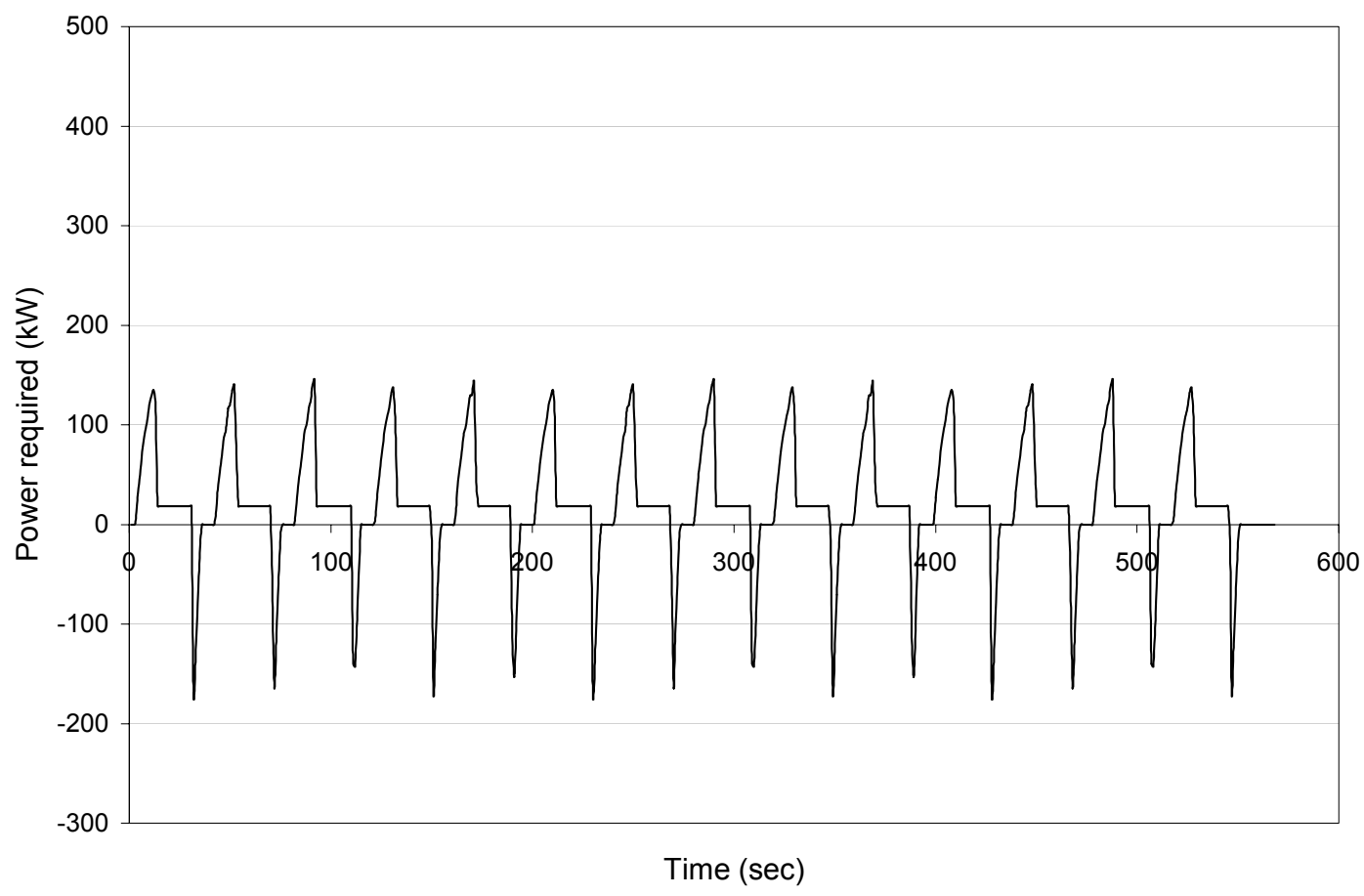

Figure 13: Power required at wheels over CBD cycle 


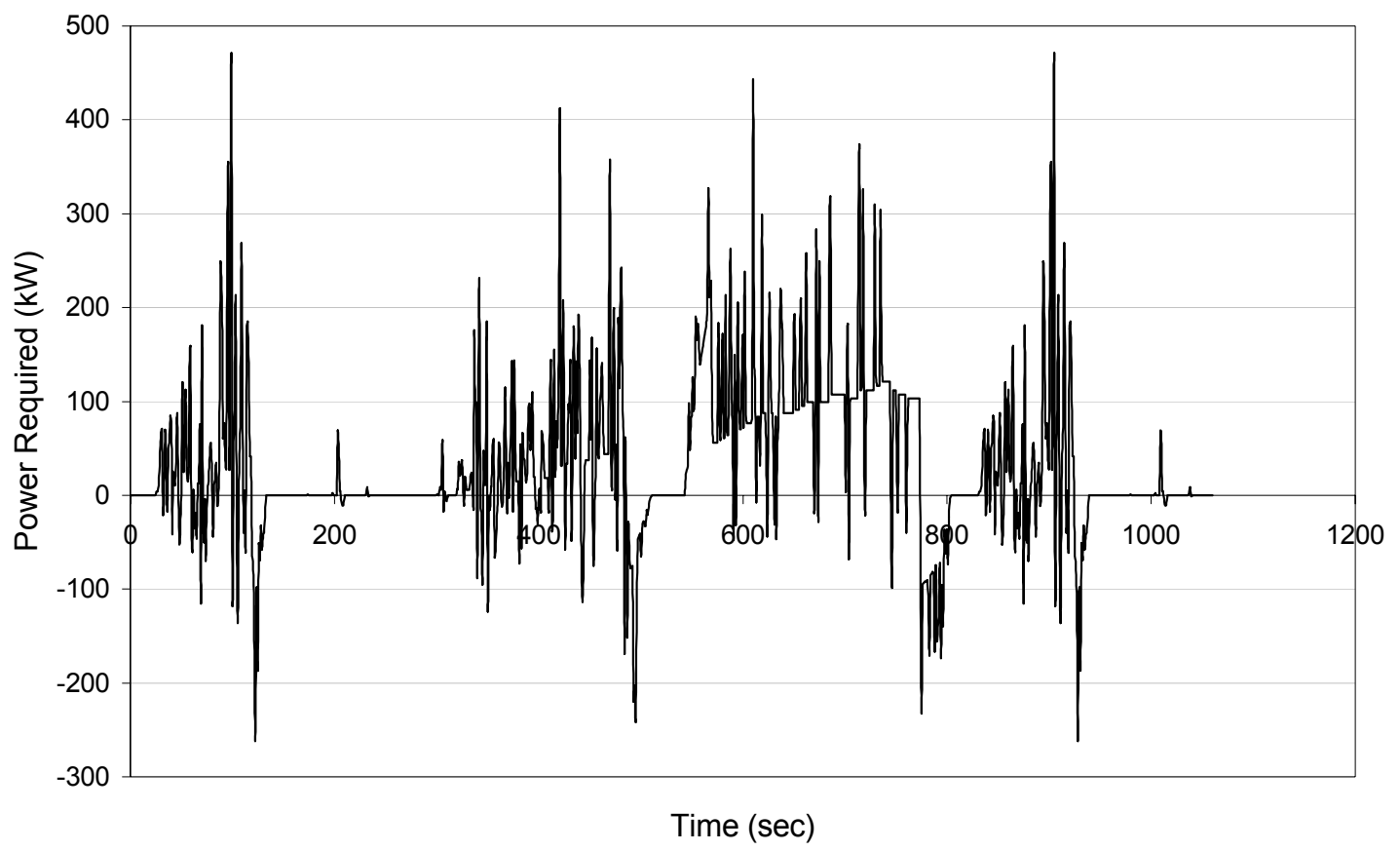

Figure 14: Power required at wheels over UDDS cycle

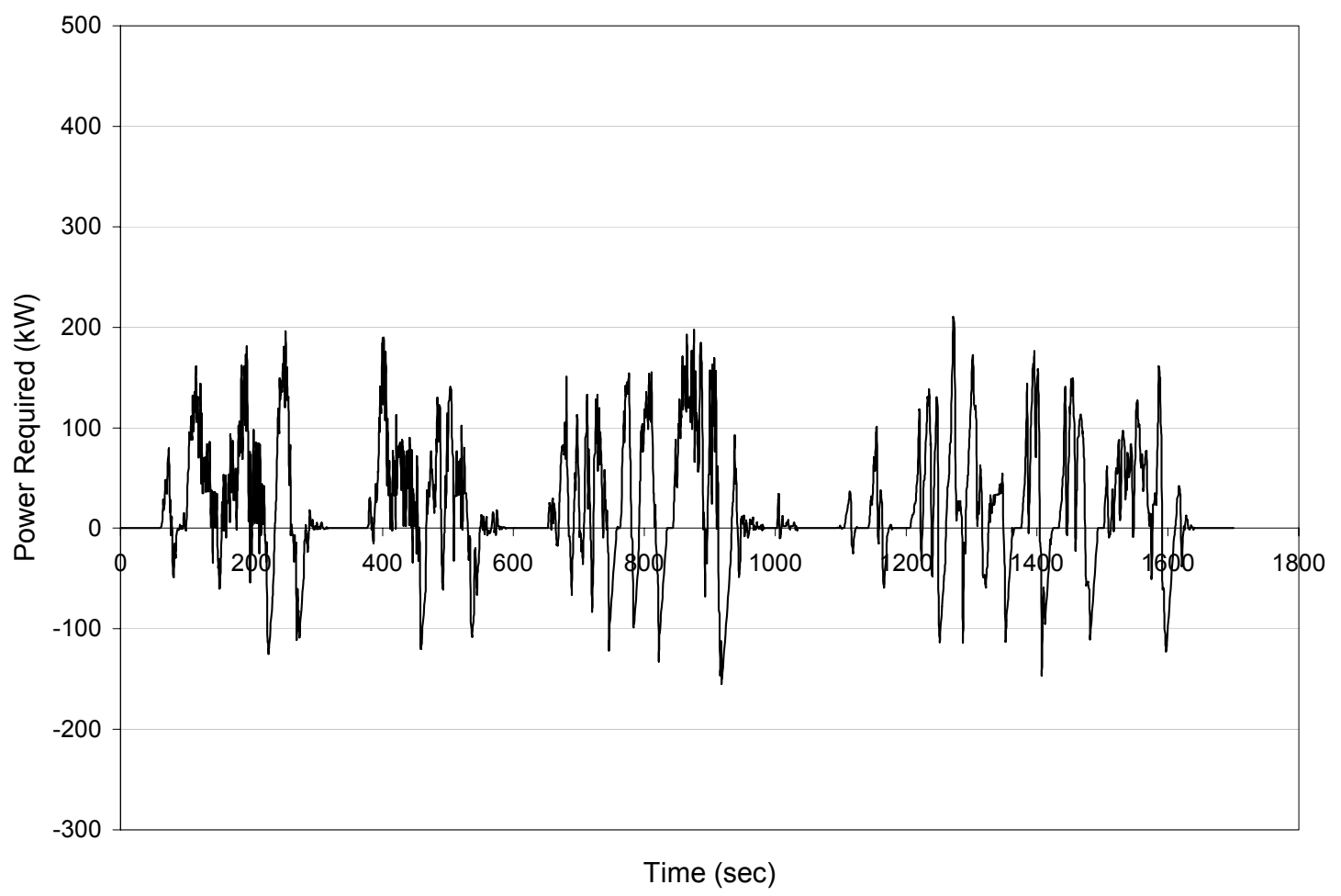

Figure 15: Power required at wheels over CSHVC 


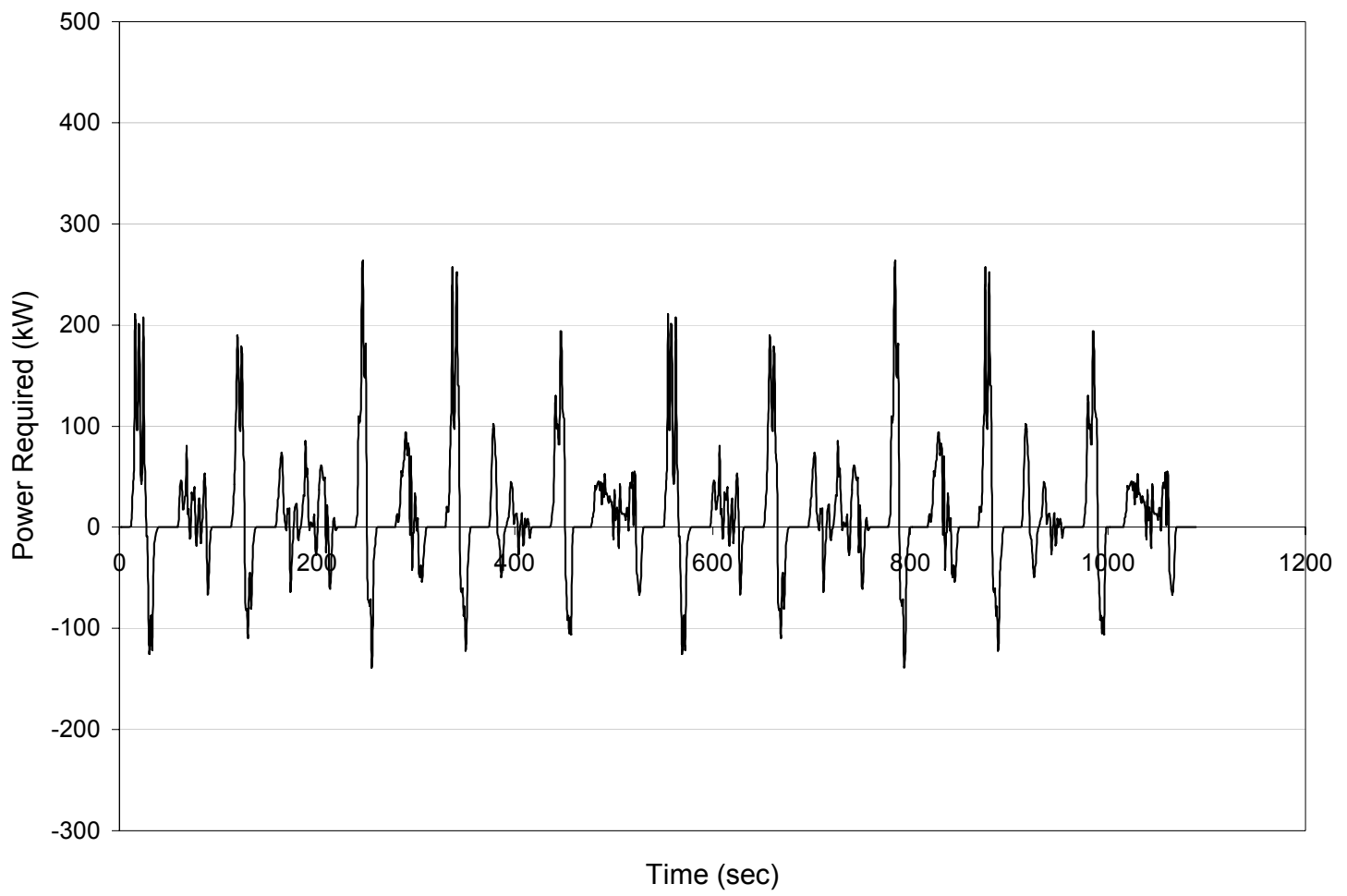

Figure 16: Power required at wheels over Manhattan cycle

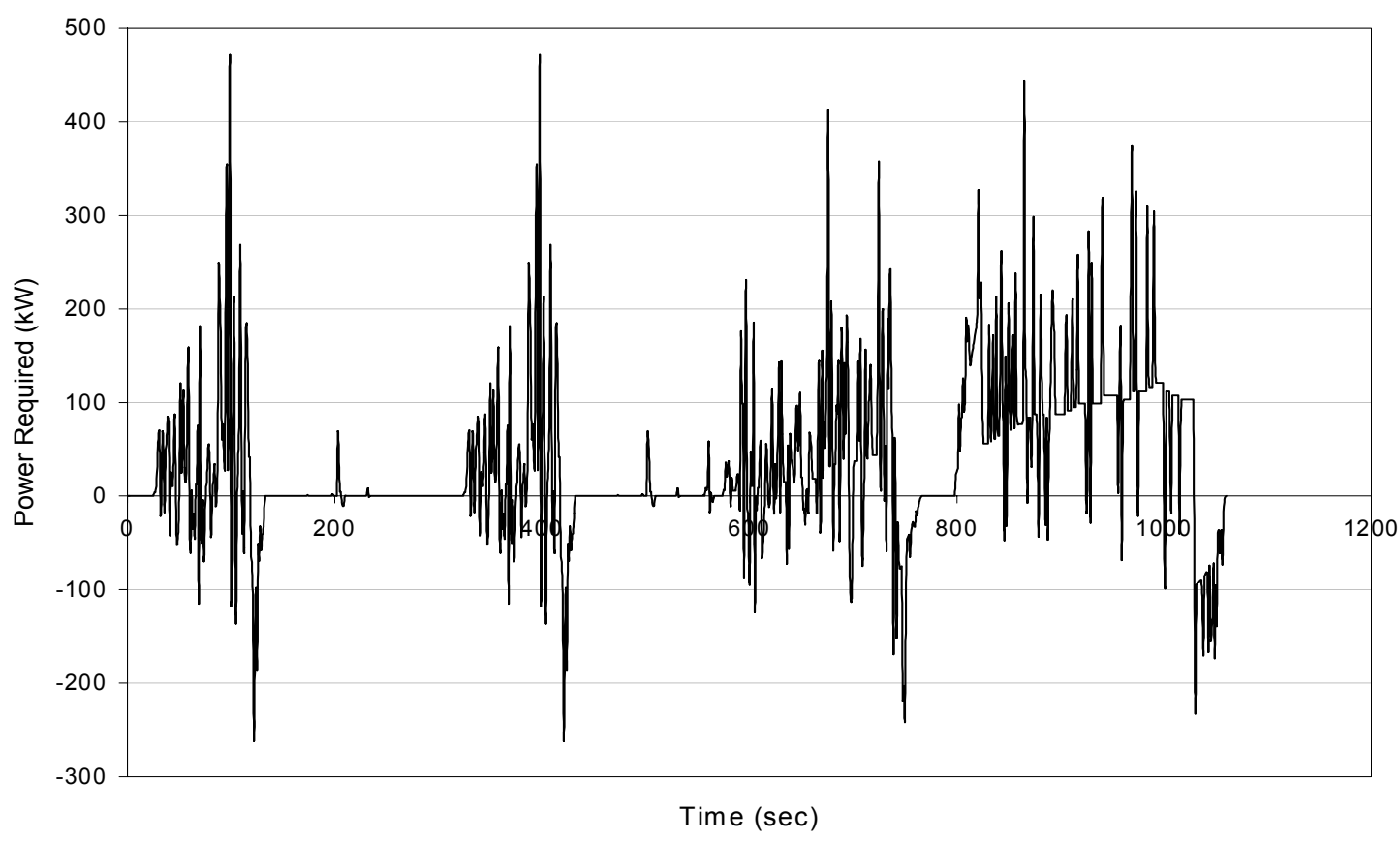

Figure 17: Power required at wheels over UDDS1 cycle 


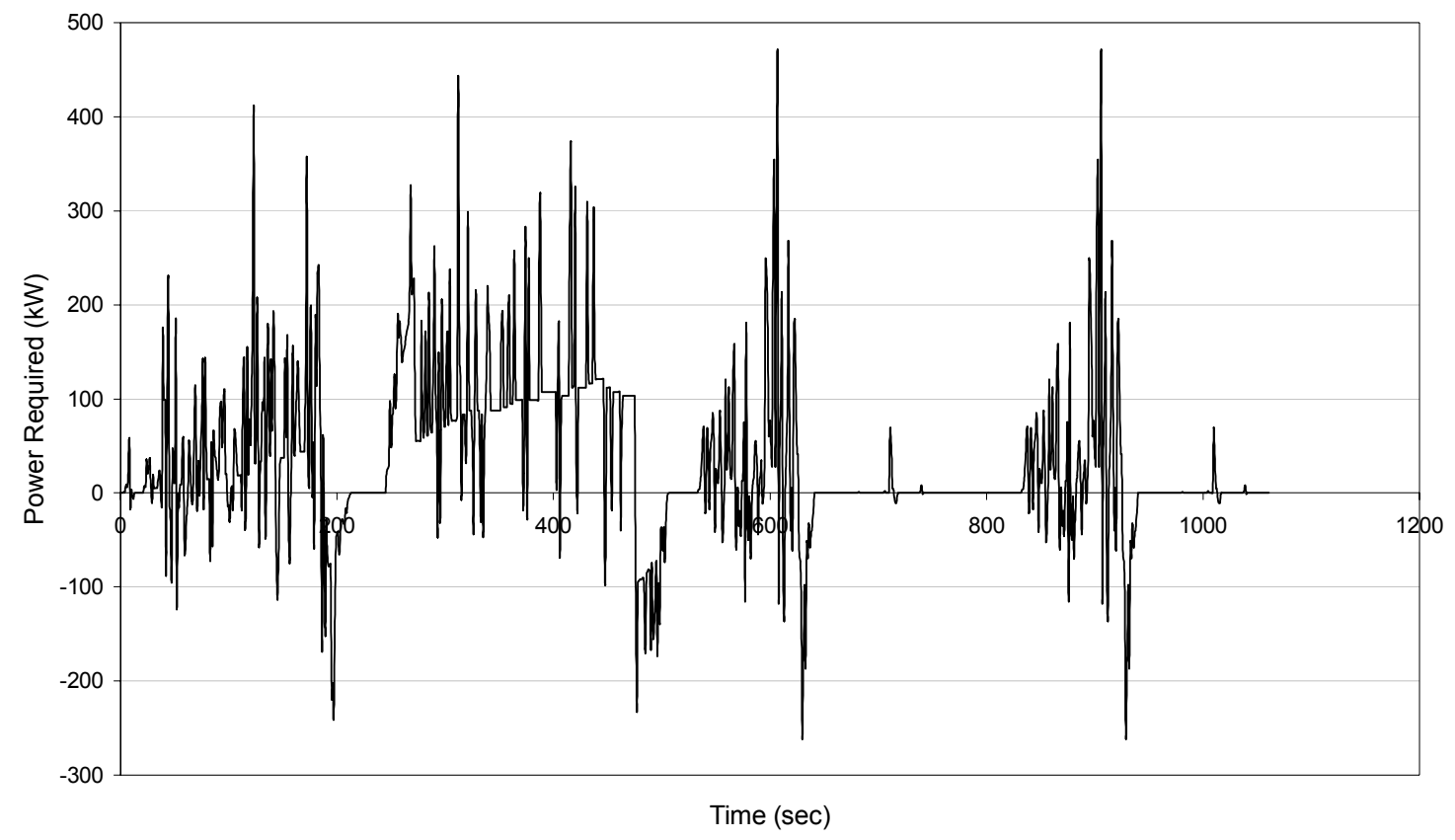

Figure 18: Power required at wheels over UDDS2 cycle

\subsection{Simulation}

Given an instantaneous power required at the vehicle wheels determined from the road load equation, the power requirements from the ICE, electric motor, and batteries can be determined as explained in section 3.2.1.

\subsubsection{Series Control Strategy}

In this thesis an equation for the power from engine has been developed based on the power required at wheels. The power at wheels is split into two, engine power and battery power. In the series HEV control strategy used in this thesis, the ICE is run at a constant percentage of the road load power, $\mathrm{C}_{1}$, and a State of Charge $(\mathrm{SoC})$ correction factor, as shown in Equation 9, while the electric motor supplies or absorbs the balance of the power required at the wheels. 


$$
P_{e}=C_{1} P_{w}+C_{2}\left(S o C_{t}-S_{o} C_{i}\right)
$$

where $\mathrm{P}_{\mathrm{e}}$ is the power from the engine,

$\mathrm{P}_{\mathrm{w}}$ is the power required at wheels,

$\mathrm{SoC}_{\mathrm{t}}$ is the target state of charge,

$\mathrm{SoC}_{\mathrm{i}}$ is the state of charge at a given time-step,

$C_{1}$ is a constant (percentage) and $C_{2}$ is a constant with units of $\frac{1}{\mathrm{sec}}$,

The SoC correction factor demands more power from the ICE when the actual SoC falls below the target value and decreases the demand on the ICE when the target SoC is exceeded. This also serves to smooth the power from the ICE relative to the road load power. The power from the ICE is delivered directly to the electric motor through a generator to avoid the losses associated with using the batteries while the electric motor draws power from or delivers power to the batteries. Additionally, the ICE can be set to run at a minimum power to account for any auxiliary loads associated with operating the vehicle. If the $\mathrm{P}_{\mathrm{e}}$ is more than the maximum engine power, then the engine supplies only the maximum power and the vehicle speed increases or acceleration decreases.

\subsubsection{Engine Efficiency}

The engine power versus speed curve can then be inserted into the simulation with the tire diameter and over-all transmission gearing defined further. At a given vehicle velocity, the rotational speed of the wheels in revolutions per minute (rpm) can be calculated using the road speed as the tangential velocity component. Using this speed and the power versus speed characteristics of the power source, the power available can be calculated for each instantaneous vehicle speed (calculated in one-second intervals). 


$$
N=\frac{60 V G R D R}{\Pi d i}
$$

where $\mathrm{N}$ is the engine speed in rpm,

$\mathrm{V}$ is the velocity of the vehicle in $\mathrm{m} / \mathrm{s}$,

GR is the gear ratio,

$\mathrm{DR}$ is the drive ratio and

di is the diameter of the tire.

The final drive ratio should be chosen to complement the operational characteristics of the power source, in that the speed and power at which the source is most likely to operate should be close to its optimum efficiency [8]. The final drive ratio is assumed to be 4.1:1. The fixed specifications of each gear ratio allows the motor speed to be calculated for each road speed in the each gear, thus allowing the maximum power available in each gear at each speed to be readily calculated.

$P_{e}=\frac{2 \prod N T}{60}$

where $\mathrm{N}$ is engine speed and $\mathrm{T}$ is engine torque.

As power from the engine is known, the torque required is calculated from this equation. From the torque versus speed trace, the efficiency of an engine for different speed bins is obtained. By curve fitting, an equation for efficiency at any given instance based on torque is obtained. The efficiency is spoken of in terms of an averaged value across a given cycle.

\subsubsection{Battery Model and Simulation}

The charge/discharge efficiency of the generic battery varies from $100 \%$ to $50 \%$ depending on the magnitude of the current into or out of the battery at any instant. The 
instantaneous current demand is calculated from the power required for the vehicle assuming a constant electric potential across the battery pack. The battery efficiency is $100 \%$ at zero battery current theoretically. As the resistance is zero, large currents would be drawn while the output would remain zero [17].

In reality, during operation of the vehicle, the voltage of the pack is a function of the current drawn from the battery pack. Typically, the higher the discharge current, the lower the battery pack voltage. However, the voltage will be kept constant for the purpose of this simulation. Using the power required to calculate current, the efficiency of the battery is allowed to vary as a function of this current. A linear equation for the efficiency is then obtained that is used in the simulation to determine the actual power required to be drawn out from the batteries to fulfill a given motor power requirement [17].

$I_{b}=\frac{P_{b}}{V_{b}}$

where $\mathrm{P}_{\mathrm{b}}$ is battery power,

$\mathrm{V}_{\mathrm{b}}$ is the voltage of battery pack and

$\mathrm{I}_{\mathrm{b}}$ is the current out of the battery.

$\eta_{b}=1-\frac{0.5}{I_{b \max }} I_{b i n s}$

where $\eta_{b}$ is the efficiency of the battery for current coming out,

$I_{b \max }$ is maximum current out of the battery and

$I_{b i n s}$ is current of the battery at a given time-step.

This equation allows the apparent battery efficiency to vary from 1 at 0 amps to 0.5 at maximum current. 
A simplified battery model was used to simulate the flow of power into and out of the batteries. Hawker Genesis G25EP batteries were chosen due to their availability and low internal resistance.

\begin{tabular}{|c|c|}
\hline Product & Hawker Genesis G25EP \\
\hline Battery Type & Lead-Acid \\
\hline Capacity & $25 \mathrm{Ah}$ \\
\hline Nominal Full-charge Voltage & $12 \mathrm{~V}$ \\
\hline Internal Resistance & $8.5 \mathrm{~m} \Omega$ \\
\hline Weight & $11 \mathrm{~kg}$ \\
\hline
\end{tabular}

Table 3: Hawker Genesis G25EP Battery Properties. [18]

Energy capacity of the batteries in joules can be calculated from

$$
E_{b}=V_{b} C_{b} N_{b}
$$

where $E_{b}$ is the energy capacity of the battery pack,

$\mathrm{V}_{\mathrm{b}}$ is the battery voltage,

$\mathrm{N}_{\mathrm{b}}$ is the number of batteries.

To achieve the 300-400V operating range of the electric motors typically used in EV and HEV operations, 27 batteries were combined resulting in a $324 \mathrm{~V}$ nominal voltage pack.

Once the power required from the electric motor is known from the control strategy, the power demand from the batteries can be calculated. The SoC of the battery pack at any instant can be calculated from,

$$
\mathrm{SoC}_{i}=\mathrm{SoC}_{i-1}-E_{b i}
$$

where $\mathrm{SoC}_{\mathrm{i}}$ is state of charge at an instance $\mathrm{i}$,

$\mathrm{SoC}_{\mathrm{i}-1}$ is the state of charge at $\mathrm{i}-1$ instance and 
$E_{b i}$ is the energy in the battery at an instance $i$.

This determines the voltage of the battery pack. From this voltage, a current draw can be calculated based on,

$$
I_{b}=\frac{P_{b}}{V_{b}}
$$

Batteries have internal resistance resulting in power losses during discharge and charging. These losses are approximated based on the current demand on the batteries.

When power is demanded from the batteries by the electric motor, this efficiency factor causes a greater power draw from the batteries.

$$
P_{b}=\frac{P_{m}}{\eta_{b}}
$$

When power is being delivered to the batteries during regenerative braking or charging while driving, the efficiency factor decreases the power available to the battery below that delivered from the electric motor.

$$
P_{b}=\eta_{b} P_{m}
$$

where $\mathrm{P}_{\mathrm{m}}$ is the motor power.

Since the power flow at the batteries is known, one Watt-sec is the energy of one watt power flowing for one second,

$$
E=P t
$$

A new $\mathrm{SoC}$ is calculated from equation 15 . Once the new $\mathrm{SoC}$ is calculated, the control strategy determines a new power level for the ICE and motor based on the road load power, the target $\mathrm{SoC}$, and the constants, $\mathrm{C}_{1}$ and $\mathrm{C}_{2}$, defined in equation 9 . 


\subsubsection{Fuel Economy}

Energy required from a fuel used in ICE must be calculated using the apparent thermal efficiency of the engine, this in turn converting that amount of energy to an equivalent volume of a given fuel using the energy of that fuel. The equations used are

$$
m_{f}=\frac{P_{r}}{H V}
$$

Here $\mathrm{HV}$ is the heating value in $\mathrm{KJ} / \mathrm{kg}$

$\mathrm{m}_{\mathrm{f}}$ is the mass flow rate of fuel and

$\mathrm{P}_{\mathrm{r}}$ is the power required.

$$
M P G=\frac{d}{\left(m_{f} d_{f}\right)}
$$

where MPG is fuel economy,

$\mathrm{d}$ is the distance covered and

$\mathrm{d}_{\mathrm{f}}$ is the density of fuel.

\subsubsection{Simulation Parameters}

Simulation parameters used for conventional heavy-duty vehicles and HEVs are tabulated in Tables 4 and 5 respectively. The various parameters used for simulations in ADVISOR, simulation models and vehicles in use are shown in these tables. The vehicle parameters for simulations in this thesis have been assumed by considering a general heavy-duty vehicle (a bus). The parameters that have been used for simulations in ADVISOR were specified in ADVISOR package. In-use vehicle parameters were taken from the West Virginia University Mobile Laboratory, where the vehicle has been tested. The parameters used are not all same for the three models in Tables 4 and 5 as the simulations were run first and then later compared to the in-use vehicle parameters. 


\begin{tabular}{|l|c|c|c|}
\cline { 2 - 4 } \multicolumn{1}{c|}{} & Simulation & Advisor & In-use \\
\cline { 2 - 4 } \multicolumn{1}{c|}{} & Model & Model & Vehicle \\
\hline Engine Type & DDC series 30 & DDC series 30 & DDC 6V-92TA \\
\hline Coeff.of Drag & 0.79 & 0.79 & 0.79 \\
\hline Front Area $\left(\mathrm{m}^{2}\right)$ & 8.05 & 7.2413 & 8.05 \\
\hline Rolling Resistance & 0.00938 & 0.008 & 0.00938 \\
\hline Vehicle Mass $(\mathrm{kg})$ & 16000 & 16000 & 14587.7 \\
\hline Transmission & Automatic & Automatic & Automatic \\
\hline Engine Rated Power $(\mathrm{kW})$ & 171 & 171 & 206 \\
\hline
\end{tabular}

Table 4:Simulation Parameters for Conventional Heavy Duty Vehicle

\begin{tabular}{|l|c|c|c|}
\cline { 2 - 4 } \multicolumn{1}{c|}{} & Simulation & Advisor & In-use \\
\cline { 2 - 4 } \multicolumn{1}{c|}{} & Model & Model & Vehicle \\
\hline Engine Type & DDC series 30 & DDC series 30 & DDC series 30 \\
\hline Coeff.of Drag & 0.79 & 0.79 & 0.79 \\
\hline Front Area $\left(\mathrm{m}^{2}\right)$ & 8.05 & 7.3506 & 7.2413 \\
\hline Rolling Resistance & 0.00938 & 0.008 & 0.008 \\
\hline Vehicle Mass $(\mathrm{kg})$ & 16000 & 16000 & 16160 \\
\hline Transmission & Automatic & Automatic & Automatic \\
\hline Engine Rated Power $(\mathrm{kW})$ & 171 & 171 & 171 \\
\hline Battery Capacity $(\mathrm{Ah})$ & 25 & 26 & 27.3 \\
\hline Motor $(\mathrm{kW})$ & 300 & 300 & 300 \\
\hline
\end{tabular}

Table 5:Simulation Parameters for Heavy-Duty Hybrid Electric Vehicle

Using these parameters simulations were run on various driving cycles for with and without auxiliary loads. Auxiliary loads include air conditioning, power steering, cooling fans, alternator, and air compressors. For heavy duty vehicles $15 \mathrm{~kW}$ load represents the load from the air conditioner and other accessories for a city bus with a full load of passengers. Auxiliary loads for the other classes were scaled according to vehicle weight. These results in an overall power required from the engine. 


\section{Simulation Results}

To allow for comparison of the results for different cycles, for each value of $\mathrm{C}_{1}$, $\mathrm{C}_{2}$ was adjusted until the battery $\mathrm{SoC}$ at the end of the cycle was equal to the initial SoC. Again for each value $\mathrm{C} 1, \mathrm{C} 2$ was adjusted until the battery SoC at the end of cycle was greater than the initial SoC. This is referred to as charge sustaining operation. Also for each value of $\mathrm{C} 1, \mathrm{C} 2$ was adjusted until the battery SoC at the end of cycle was less than the initial SoC. This is referred to as charge depleting operation. $\mathrm{C}_{2}$ governs the $\mathrm{SoC}$ dependence of the engine. If $\mathrm{C}_{2}$ is high, the engine power will increase a large amount relative to the difference between the target $\mathrm{SoC}$ and the $\mathrm{SoC}_{\mathrm{i}}$. When the $\mathrm{SoC}$ climbs above the target $\mathrm{SoC}$, the $\mathrm{C}_{2}$ correction factor decreases the engine power to increase power demand on the electric motor and batteries. When the $\mathrm{SoC}$ falls below the target SoC, the correction factor increases the engine power reducing demand on the batteries and, in some instances, providing energy to the batteries through charging while driving. The initial SoC was set at $60 \%$ and $90 \%$ for all simulations. If the initial SoC were set at $100 \%$, not only would this be an unrealistic expectation for a charge-sustaining hybrid, but also there would be no capacity for recapturing regenerative braking energy until the batteries had been somewhat depleted. 


\begin{tabular}{|c|c|c|c|c|c|c|}
\hline & \multicolumn{6}{|c|}{ Conventional Vehicle Data } \\
\hline & w/o auxil & iliary load & with auxil & liary load & w/o auxiliary & with auxiliary \\
\hline & Simulation & Advisor & Simulation & Advisor & load & load \\
\hline Cycle & \multicolumn{4}{|c|}{ Fuel Economy (MPG) } & $\%$ Diff in MPG & $\%$ Diff in MPG \\
\hline CBD & 4.85 & 4.2 & 3.39 & 3.8 & 15.54 & -10.63 \\
\hline Manhattan & 3.68 & $\overline{3.2}$ & 2.38 & 2.9 & 18.93 & -17.68 \\
\hline CSHVC & 5.5 & 5.3 & 3.96 & 5 & 3.86 & -20.7 \\
\hline UDDS & 4.96 & 3.8 & 3.89 & 3.7 & 1.16 & -5.29 \\
\hline UDDS1 & 4.96 & 3.8 & 3.86 & 3.7 & 30.52 & 4.56 \\
\hline UDDS2 & 4.96 & 3.8 & 3.89 & 3.7 & 30.52 & 5.27 \\
\hline
\end{tabular}

Table 6: Fuel Economy for Conventional Vehicles

Table 6 shows fuel economy data of a conventional vehicle for the thesis simulation model and ADVISOR model over different driving cycles with and without auxiliary loads. There is difference in fuel economy data for thesis simulation model and ADVISOR model as shown in Table 6. This is due to the difference in control strategy used. In simulation model engine is always on, where as in ADVISOR model engine is turned off when required. The percent differences in the fuel economy of UDDS cycle and CSHVC, without auxiliary load are very less of $1.16 \%$ and $3.87 \%$ respectively. Further these simulation results of fuel economy were compared with those of actual inuse vehicles. The fuel economy data of in-use conventional transit bus for CBD cycle and UDDS cycle were taken form the West Virginia University Mobile Laboratory. The fuel economy of in-use transit bus tested in West Virginia University Mobile Laboratory over CBD cycle (Test ID 1113) was 3.14 mpg and 5.02 mpg for UDDS cycle (Test ID 1213). When comparison is done for thesis simulation model and actual in-use vehicles then it is $35.2 \%$ for CBD cycle and $1.14 \%$ for UDDS cycle. When a similar comparison is done for ADVISOR simulations and actual in-use vehicles, the percent difference is found to be $25.2 \%$ for CBD cycle and $-28.6 \%$ for UDDS cycle. 
In-use hybrid vehicle recorded a fuel economy of $3.5 \mathrm{mpg}$ (Test ID 3480) over Manhattan cycle and 4.04 mpg (Test ID 3462) over CBD cycle. When the in-use fuel economy figures for heavy-duty hybrid vehicles were compared to the ADVISOR simulation results for the Manhattan Cycle, they agree. Fuel economy of the hybrid vehicle in ADVISOR model over CBD cycle varied by $8.9 \%$ with that of the in-use hybrid electric vehicle. Difference of $33.5 \%$ and $23 \%$ in fuel economy over Manhattan and CBD cycles respectively is found for thesis simulation model and actual in use HEVs. Table 7 shows fuel economy data for thesis simulation model and ADVISOR model over various driving cycles.

\begin{tabular}{|c|c|c|c|c|c|c|}
\hline & \multicolumn{6}{|c|}{ Heavy-Duty Hybrid Electric Vehicle Data } \\
\hline & w/o auxil & iliary load & with auxil & iary load & w/o auxiliary & with auxiliary \\
\hline & Simulation & Advisor & Simulation & Advisor & load & load \\
\hline Cycle & \multicolumn{4}{|c|}{$\begin{array}{ll}\text { Fuel Economy (MPG) } \\
\end{array}$} & $\%$ Diff in MPG & \% Diff in MPG \\
\hline CBD & 5.24 & 4.4 & 4.78 & 4 & 19.27 & 19.6 \\
\hline Manhattan & 5.26 & 3.5 & 2.67 & 3.1 & 50.34 & -13.7 \\
\hline CSHVC & 6.17 & 6.3 & 4.94 & 5.8 & 1.92 & -14.75 \\
\hline UDDS & 6.01 & 5.8 & 5.24 & 5.5 & 3.7 & -4.65 \\
\hline UDDS1 & 5.24 & 5.8 & 4.4 & 5.4 & -9.5 & -18.446 \\
\hline UDDS2 & 5.45 & 5.7 & 4.65 & 5.4 & -4.26 & -13.75 \\
\hline
\end{tabular}

Table 7: Fuel Economy from simulations for Series HEVs

Figures 19 to 30 show power required at the wheels and engine power required over various driving cycles for a heavy-duty HEV with and without auxiliary loads. 


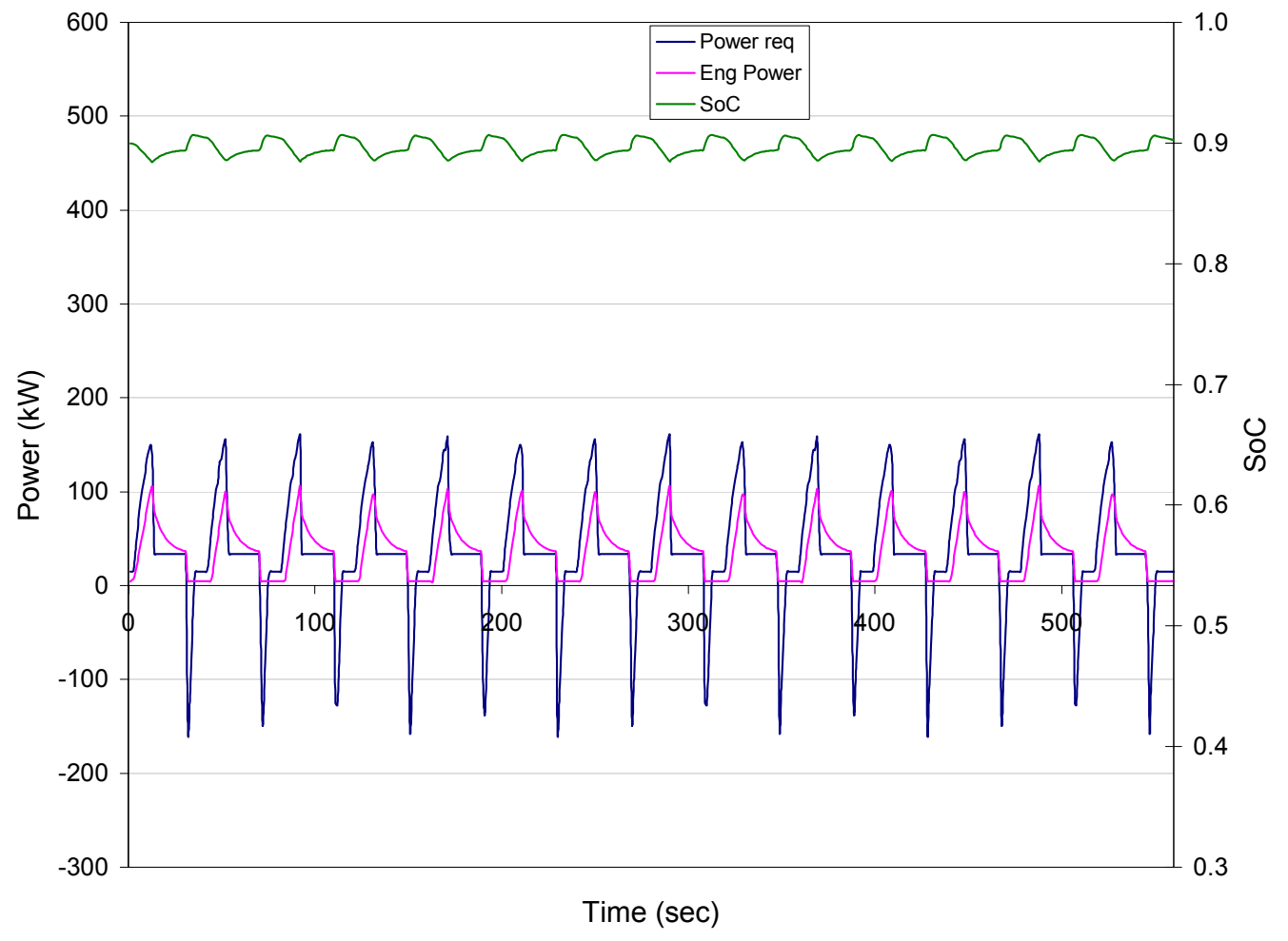

Figure 19: HEV on CBD cycle with auxiliary load for $\mathrm{Cl}=0.3$ and $\mathrm{SoC}$ at $90 \%$

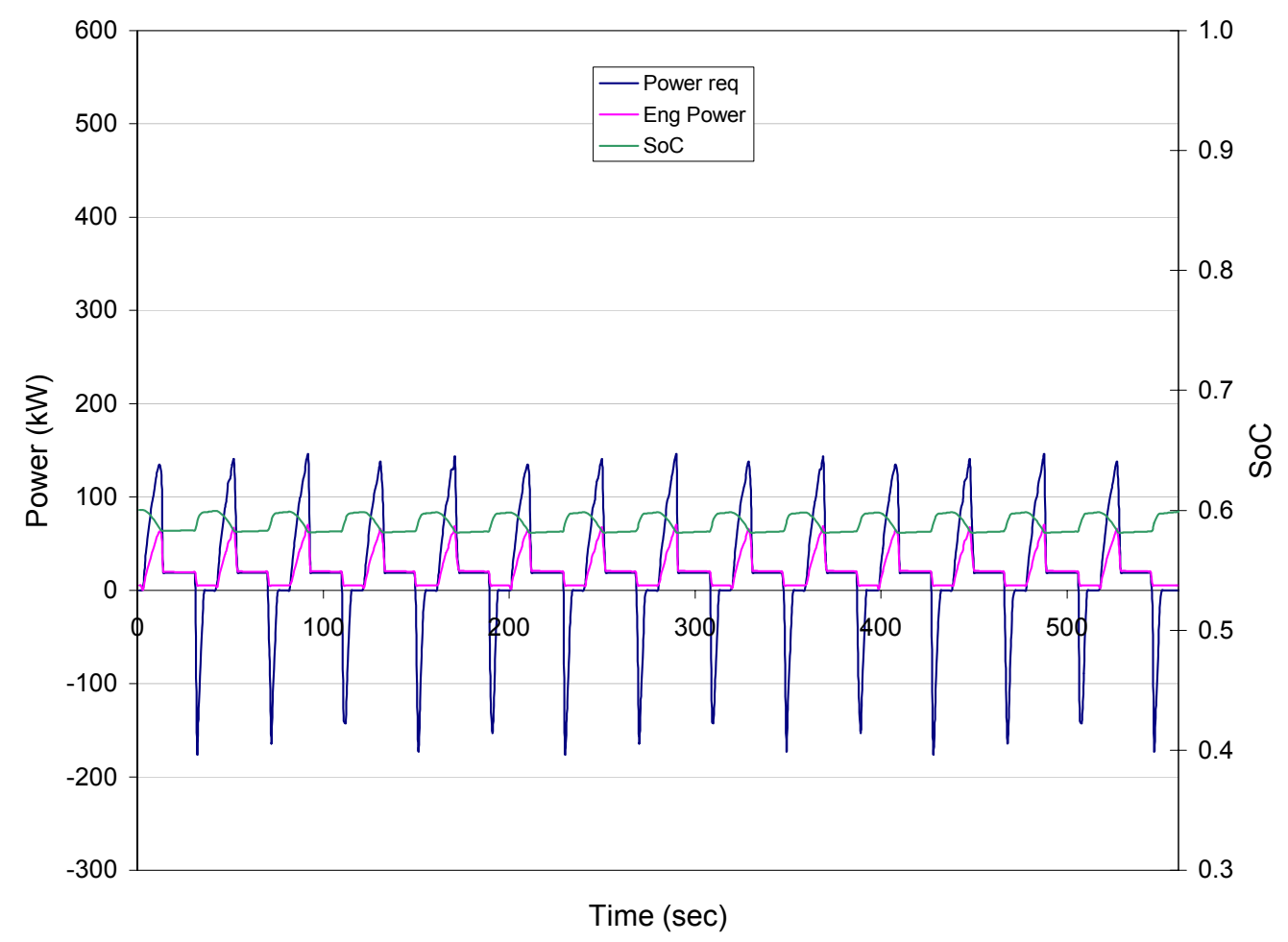

Figure 20: HEV on CBD cycle without auxiliary load for $\mathrm{C} 1=0.4$ and SoC at $60 \%$ 
In the Figures 19 and 20, the peaks of power required vary in amplitude because of the non-uniform rate of acceleration and deceleration for a CBD cycle. When a vehicle is following a speed vs time trace, depending on the driver, there would be different rates of acceleration and deceleration. Here, in this thesis, simulations were done for heavy duty HEVs with and without auxiliary loads. For different state of charge, beginning at $60 \%$ and $90 \%$ of the power required at wheels, engine power and state of charge are plotted. The simulations are run based on the control strategy given in equation 9 for particular $\mathrm{C} 1$ and $\mathrm{C} 2$ values.

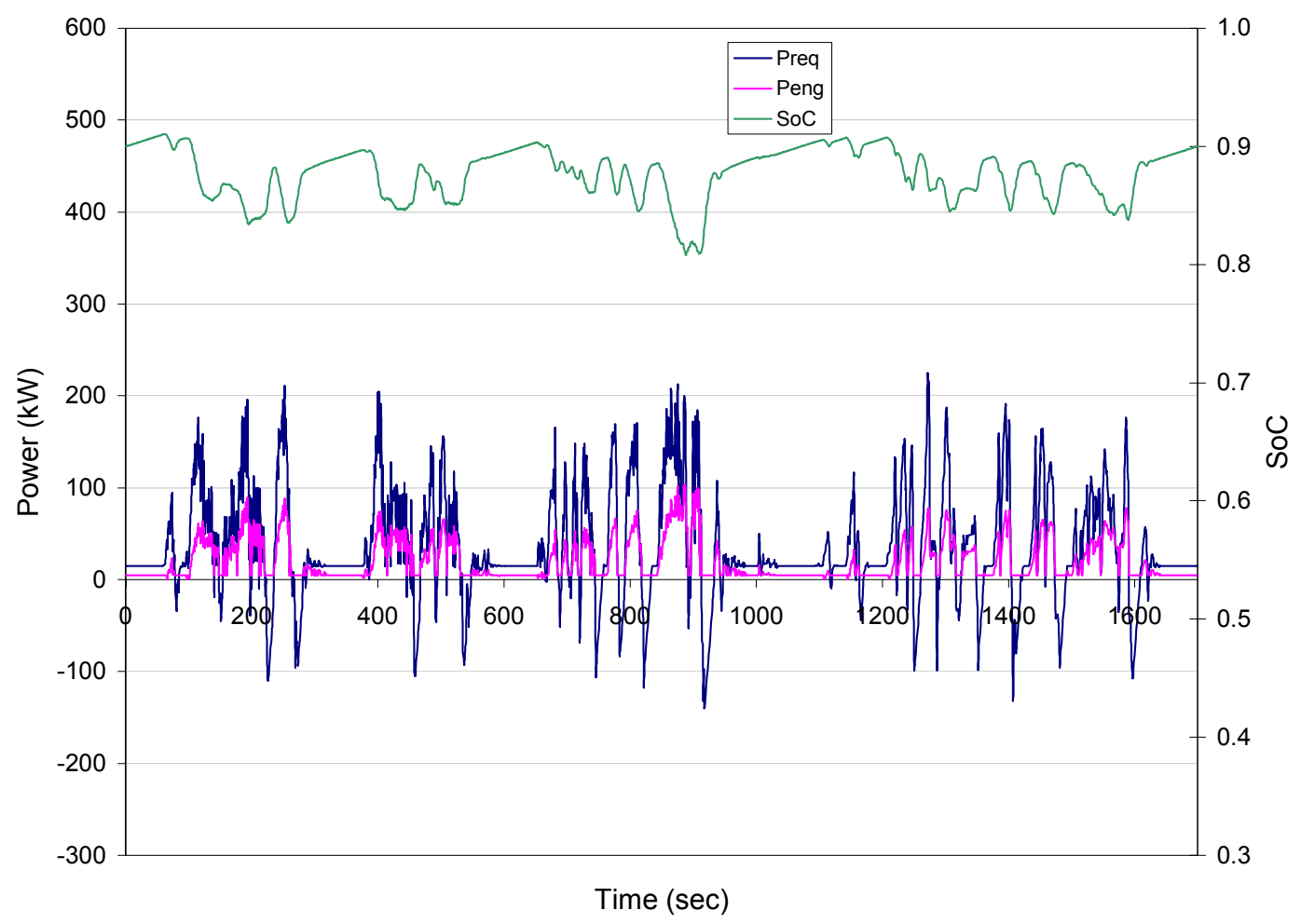

Figure 21: HEV on CSHVC with auxiliary load for $\mathrm{C1}=0.3$ and $\mathrm{SoC}$ at $90 \%$ 


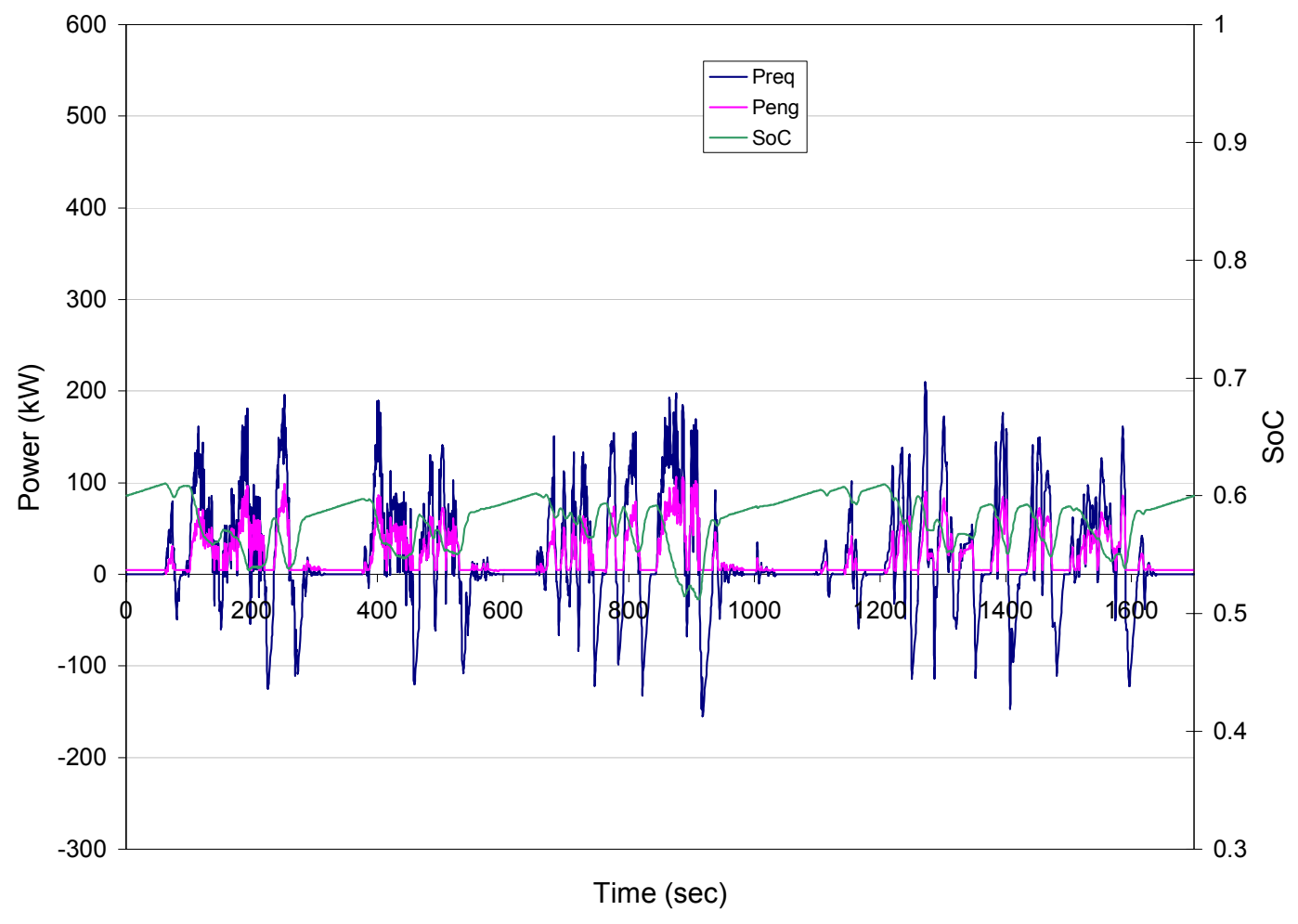

Figure 22: HEV on CSHVC without auxiliary load for C1 0.4 and SoC at $60 \%$

In Figures 21 and 22 the power required at the wheels, engine power and state of charge over CSHVC are shown. The control strategy used, allows the battery charge to fluctuate between $90 \%$ and $60 \%$ of SoC. The upper and lower limits being $90 \%$ and $60 \%$ of total capacity of the battery pack. Figures 23 and 24 show the power required at wheels, engine power and SoC for both with and without auxiliary load over the Manhattan cycle. 


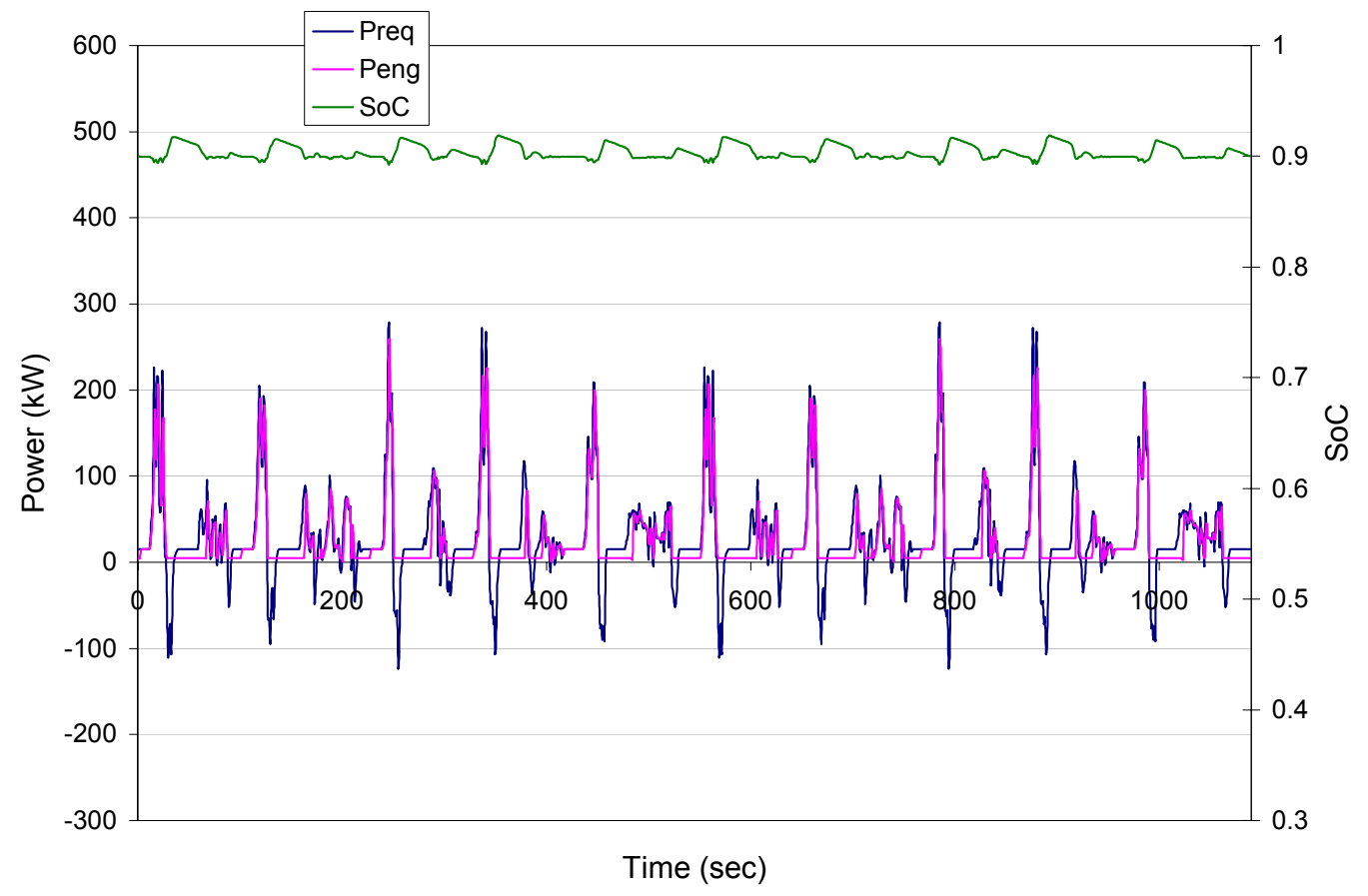

Figure 23: HEV on Manhattan cycle with auxiliary load for $\mathrm{C} 1=0.3$ at $90 \% \mathrm{SoC}$

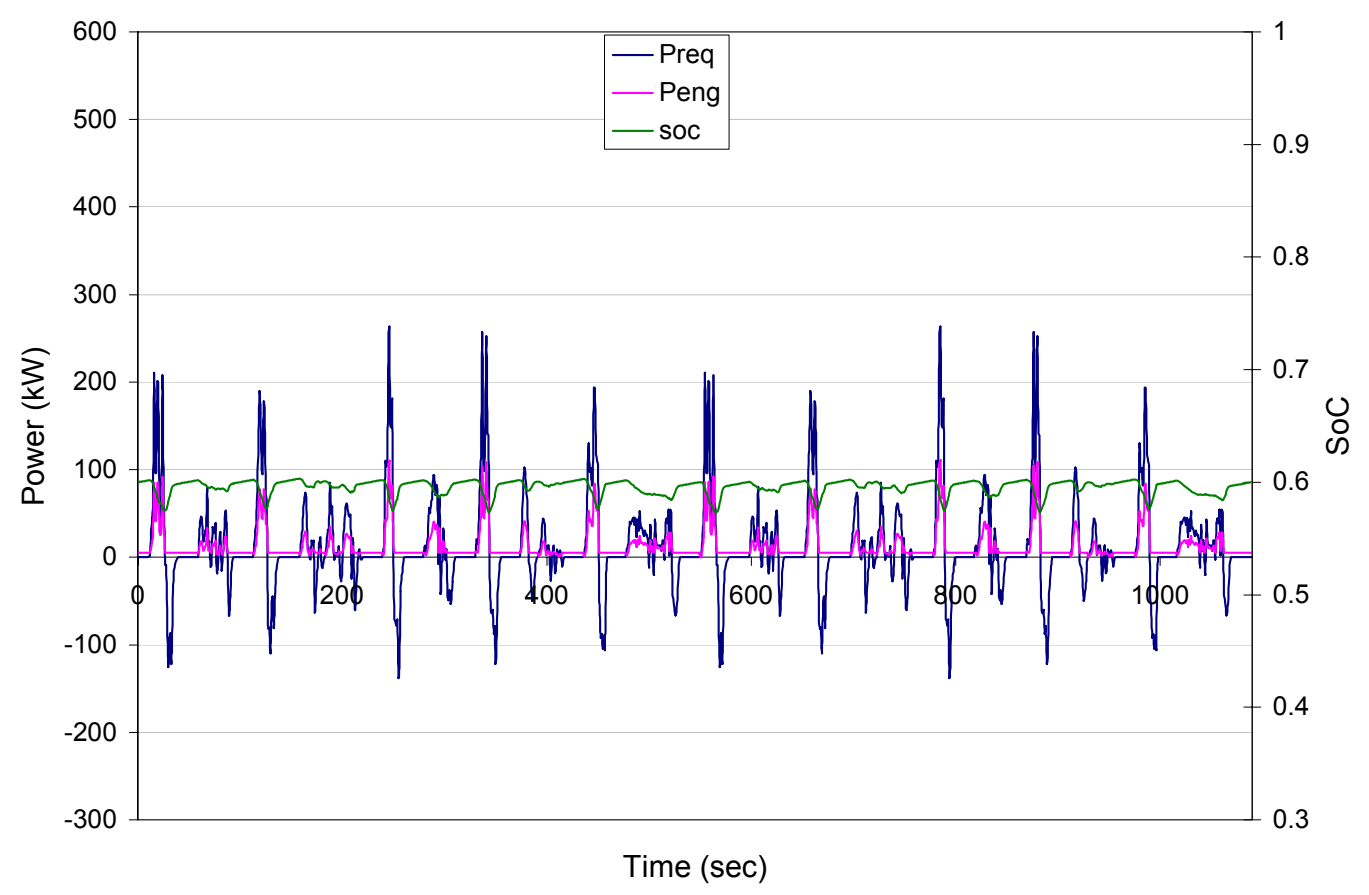

Figure 24: HEV on Manhattan cycle without auxiliary load for $\mathrm{C} 2=0.4$ at $60 \%$ SoC 
Figures 25, and 26 show the plots of power required at wheels, engine power and $\mathrm{SoC}$ over the UDDS cycle. Figures 27, 28, 29 and 30 show the power required, engine power and SoC over cycles UDDS1 and UDDS2.

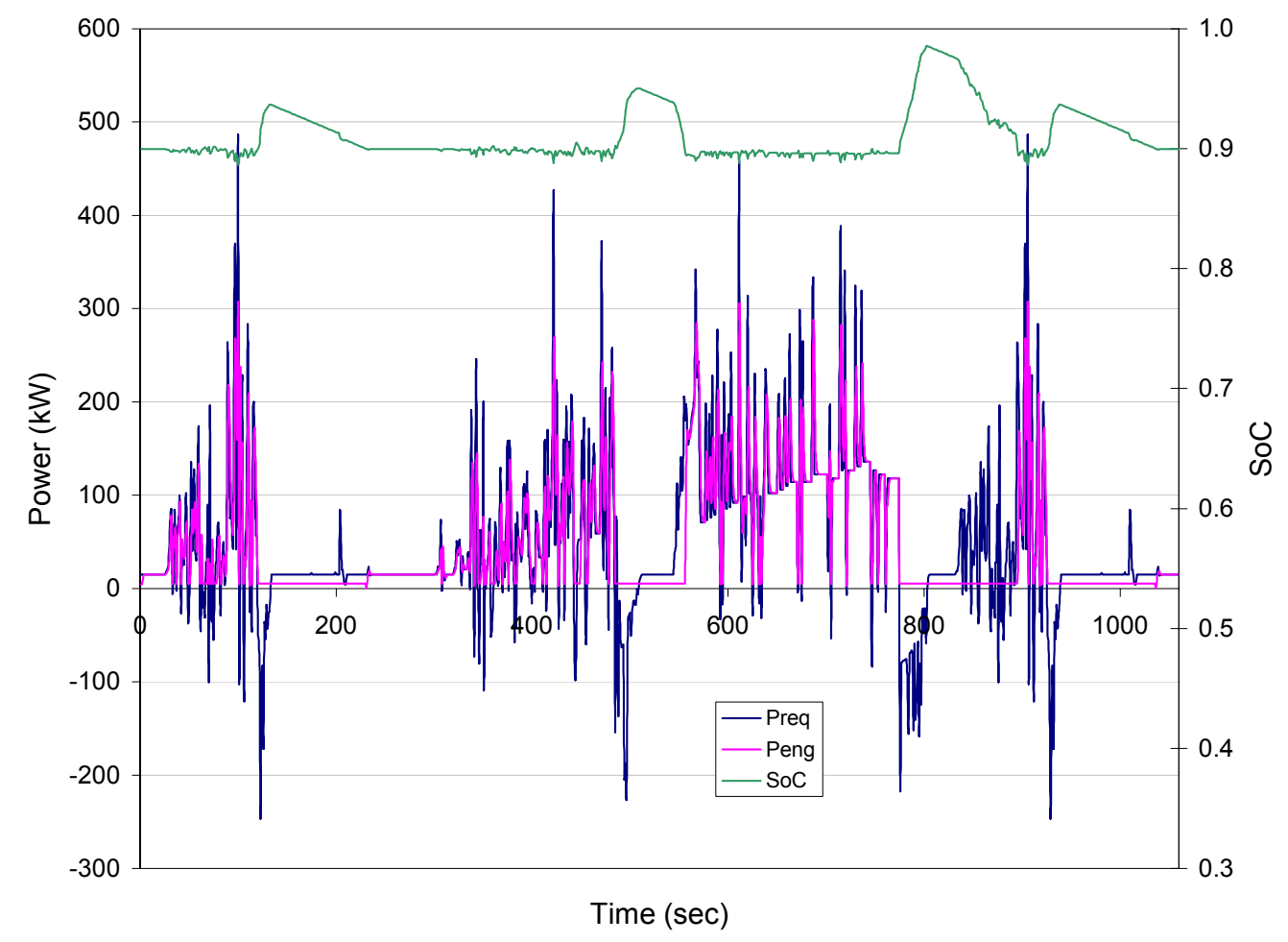

Figure 25: HEV on UDDS with auxiliary load for $\mathrm{C} 1=0.3$ 


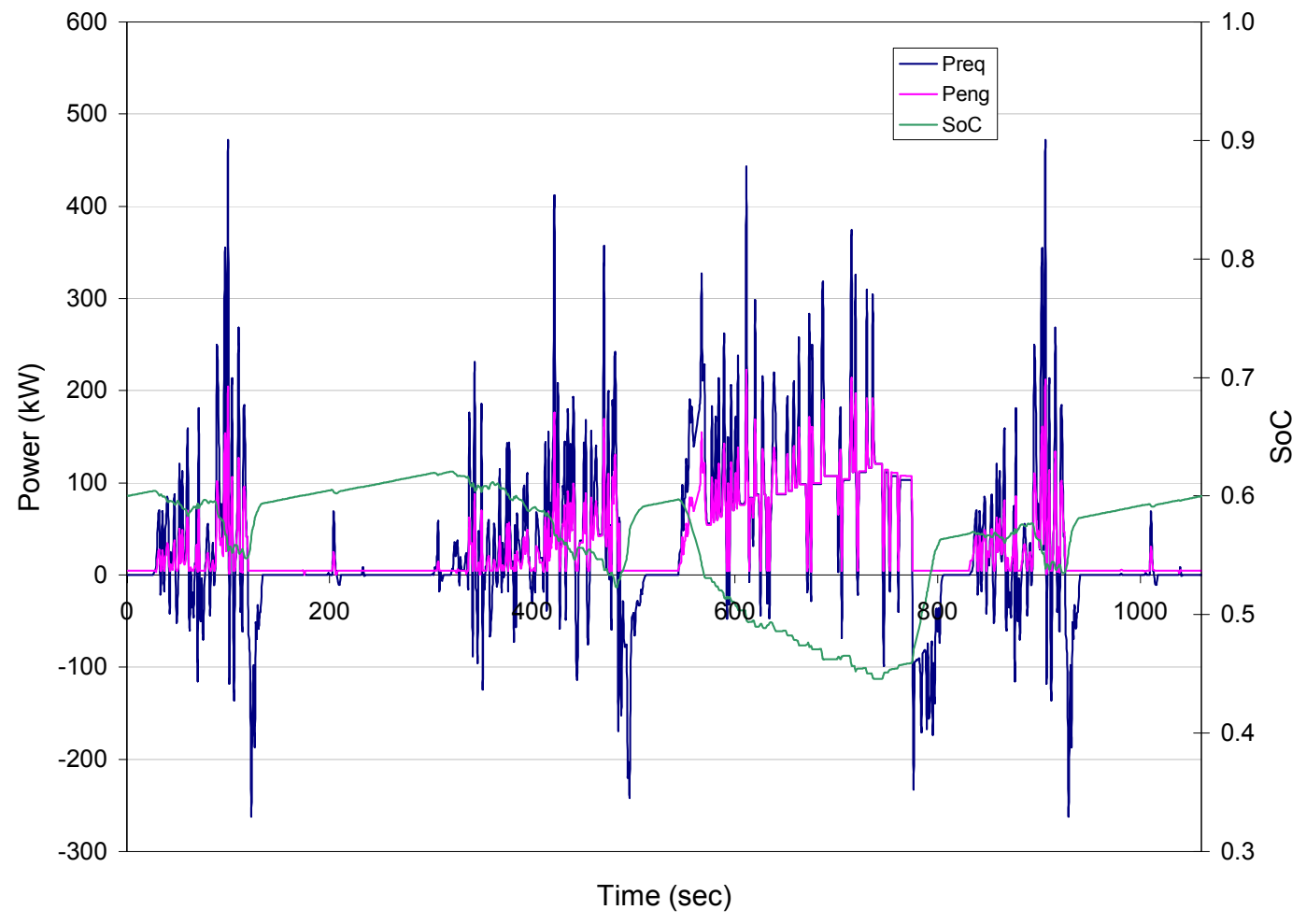

Figure 26: HEV on UDDS cycle without auxiliary load for $\mathrm{C} 1=0.4$ and $\mathrm{SoC}$ at $60 \%$

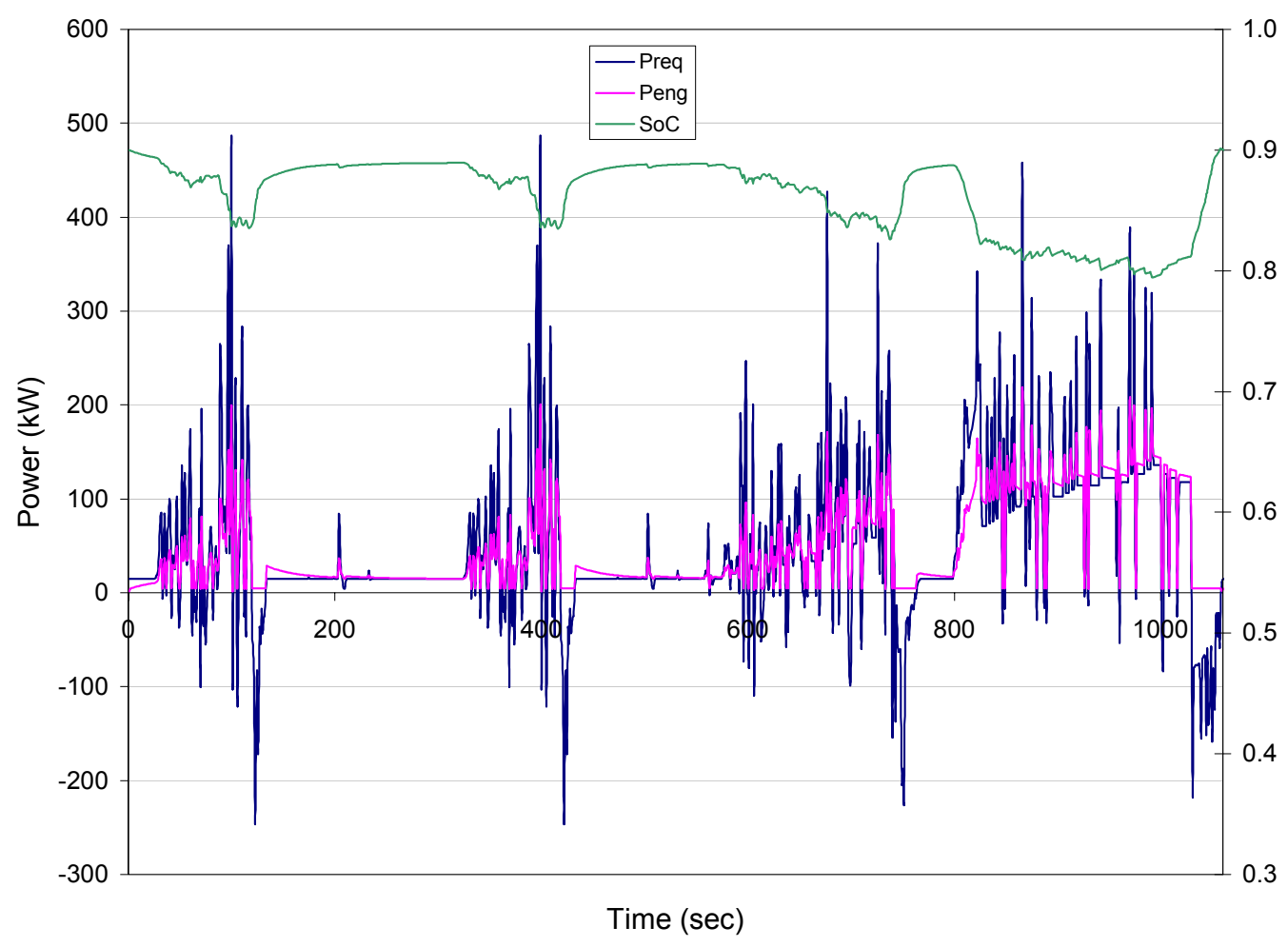

Figure 27: HEV on UDDS1 cycle with auxiliary load for $\mathrm{C} 1=0.3$ and $\mathrm{SoC}$ at $90 \%$ 


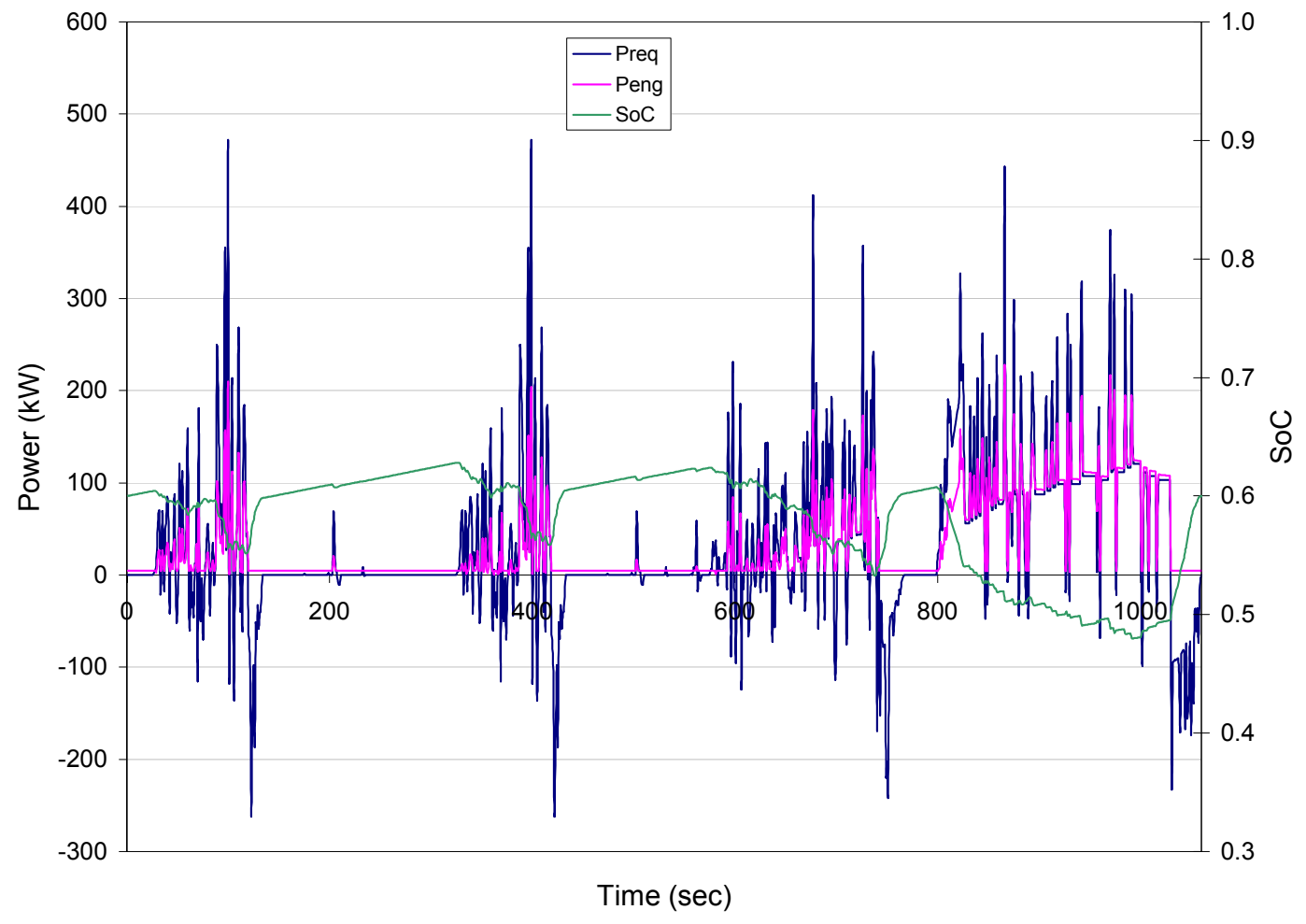

Figure 28: HEV on UDDS1 cycle without auxiliary load for $\mathrm{C} 1=0.4$ showing $\mathrm{SoC}$ at $60 \%$

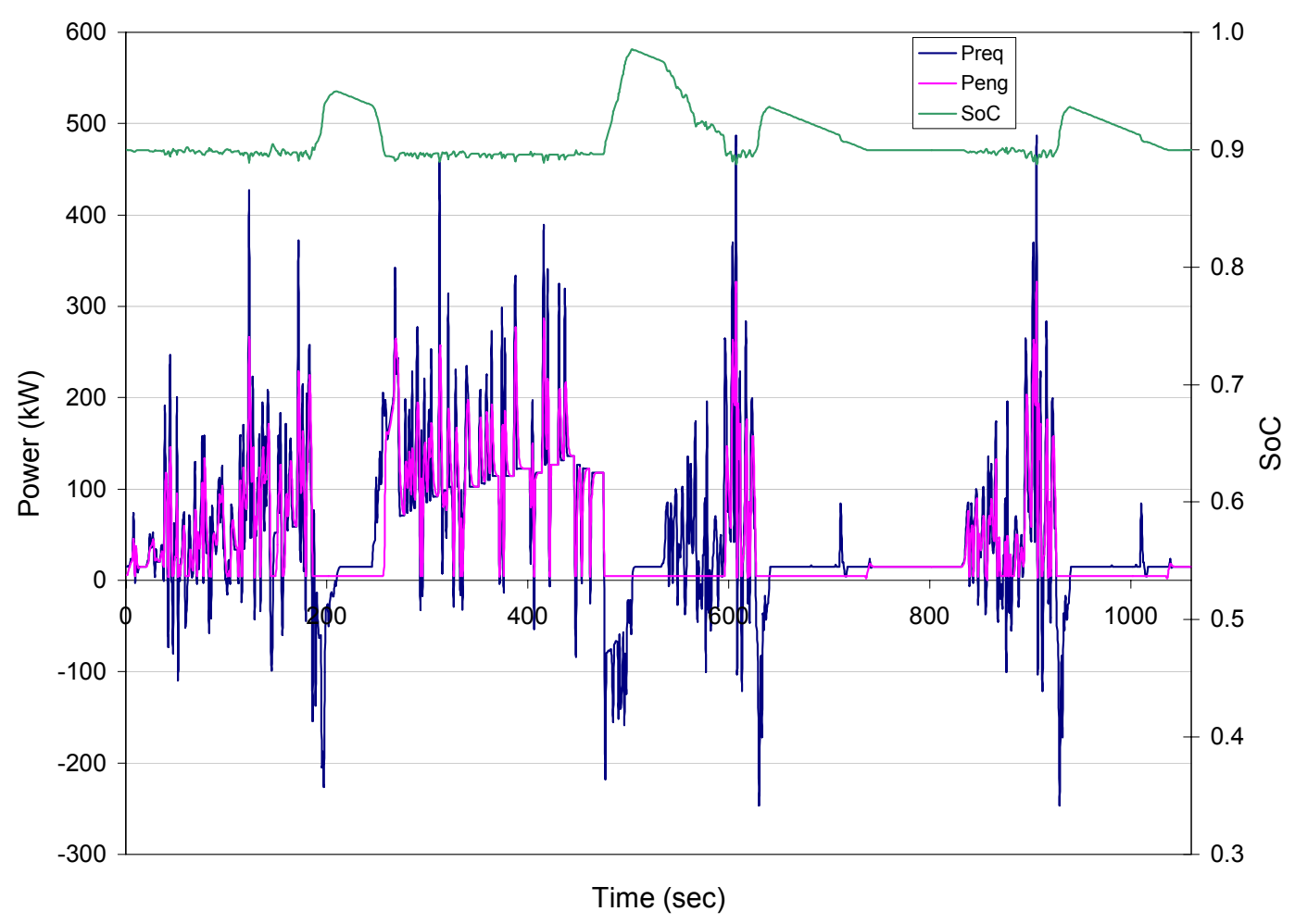

Figure 29: HEV on UDDS2 cycle with auxiliary load for $\mathrm{C} 1=0.4$ showing engine power and SoC 


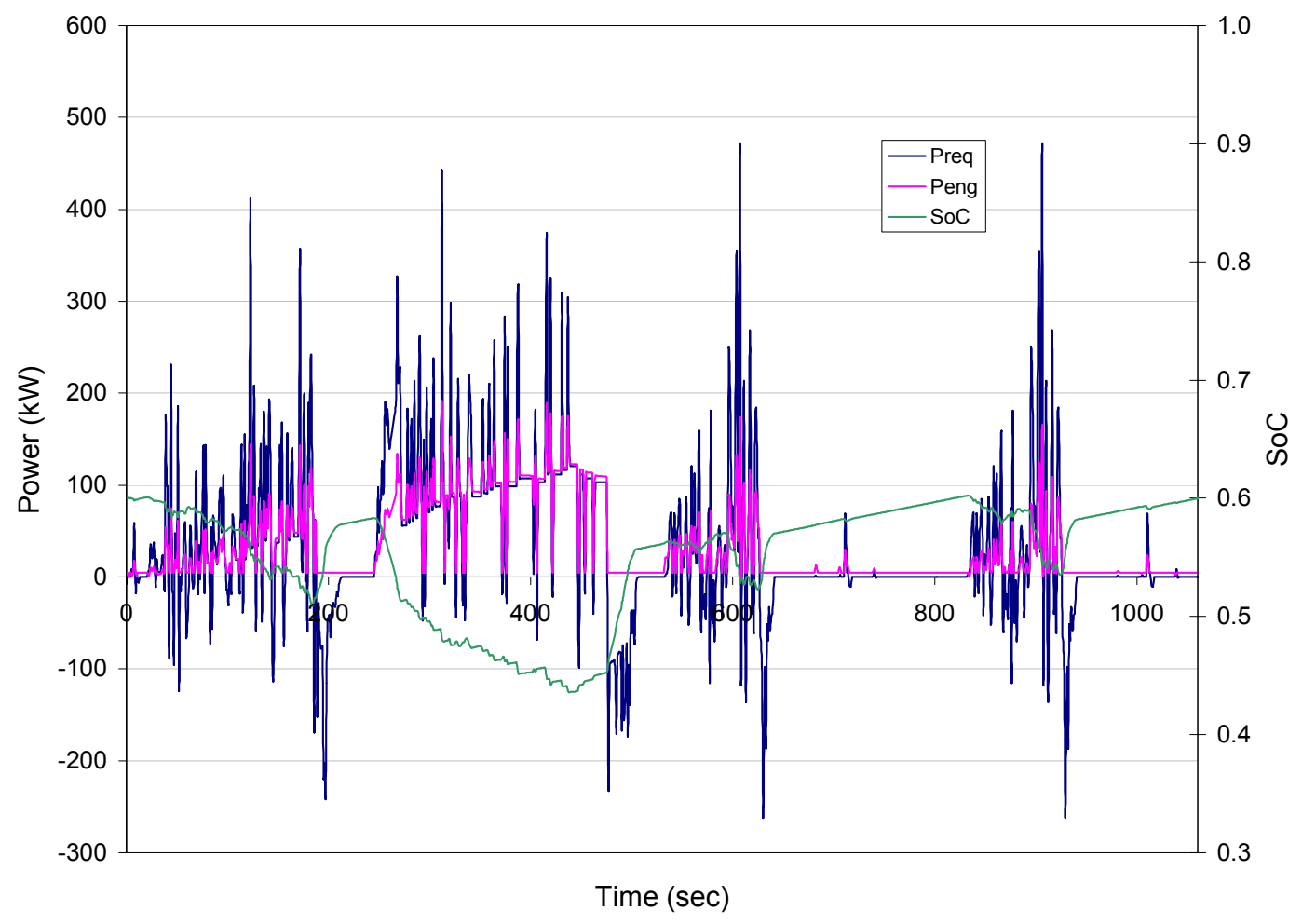

Figure 30: HEV on UDDS2 cycle without auxiliary load for $\mathrm{C} 1=0.3$ showing engine power and SoC

\subsection{Optimization}

Figures 31 through 36 show the variation of fuel economy with $C_{1}$ for the various driving cycles. In all of the vehicles, the addition of auxiliary loads was extremely detrimental to the fuel economy. Generally, the fuel economy with auxiliary loads was half the fuel economy without auxiliary loads. This is due to requiring the engine to run inefficiently at low power levels throughout long periods of the cycles. The fuel economy of HEV with and without auxiliary loads over different cycles is shown in Figures 31 through 36 . When the vehicle is stopped without auxiliary loads, HEVs commonly allow the engine to shut off greatly reducing fuel economy over many cycles. The addition of auxiliary loads not only removes this option but also increases the fuel consumption during these periods. 


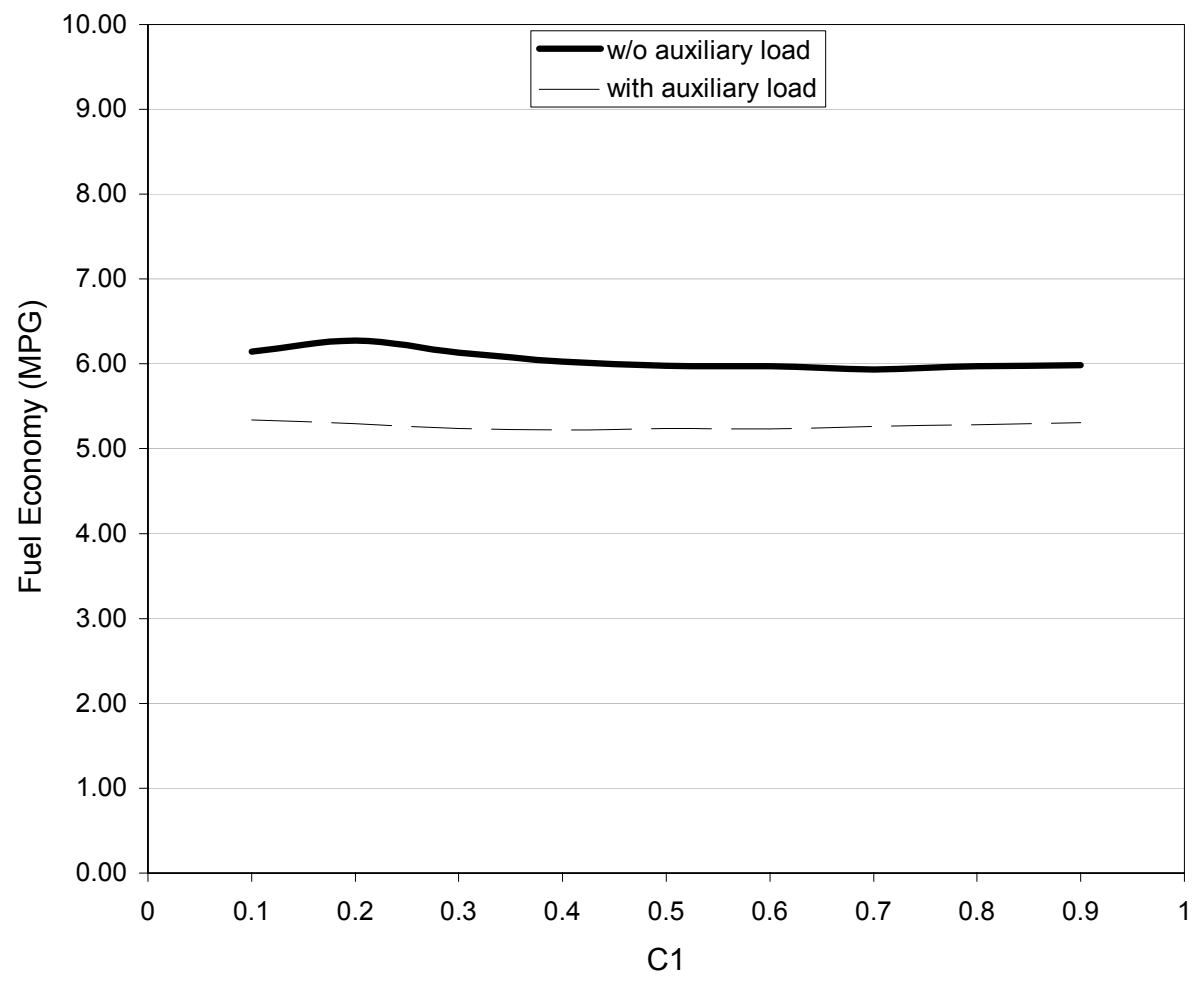

Figure 31: HEV Fuel Economy on CBD cycle

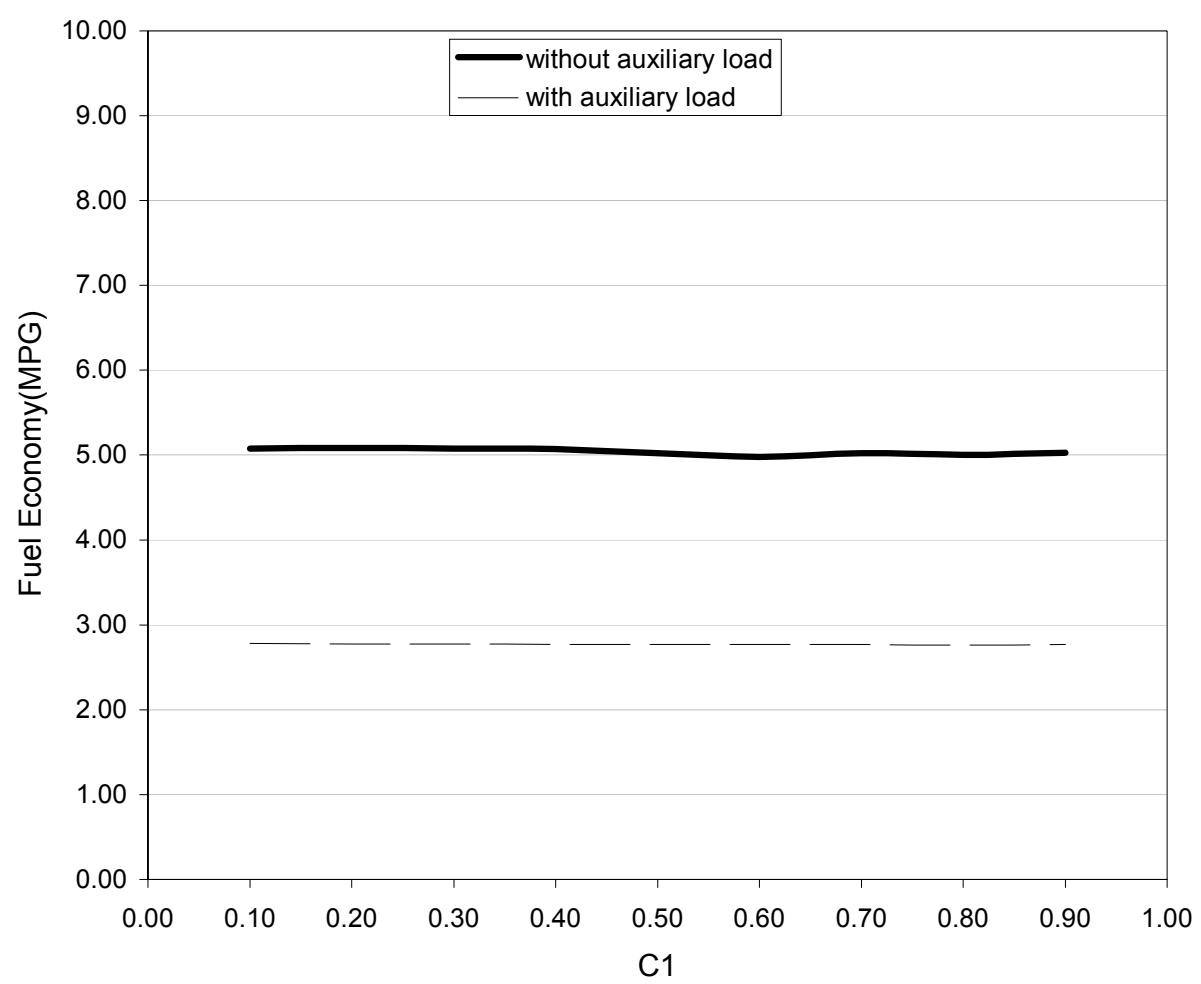

Figure 32: HEV Fuel Economy on Manhattan cycle 


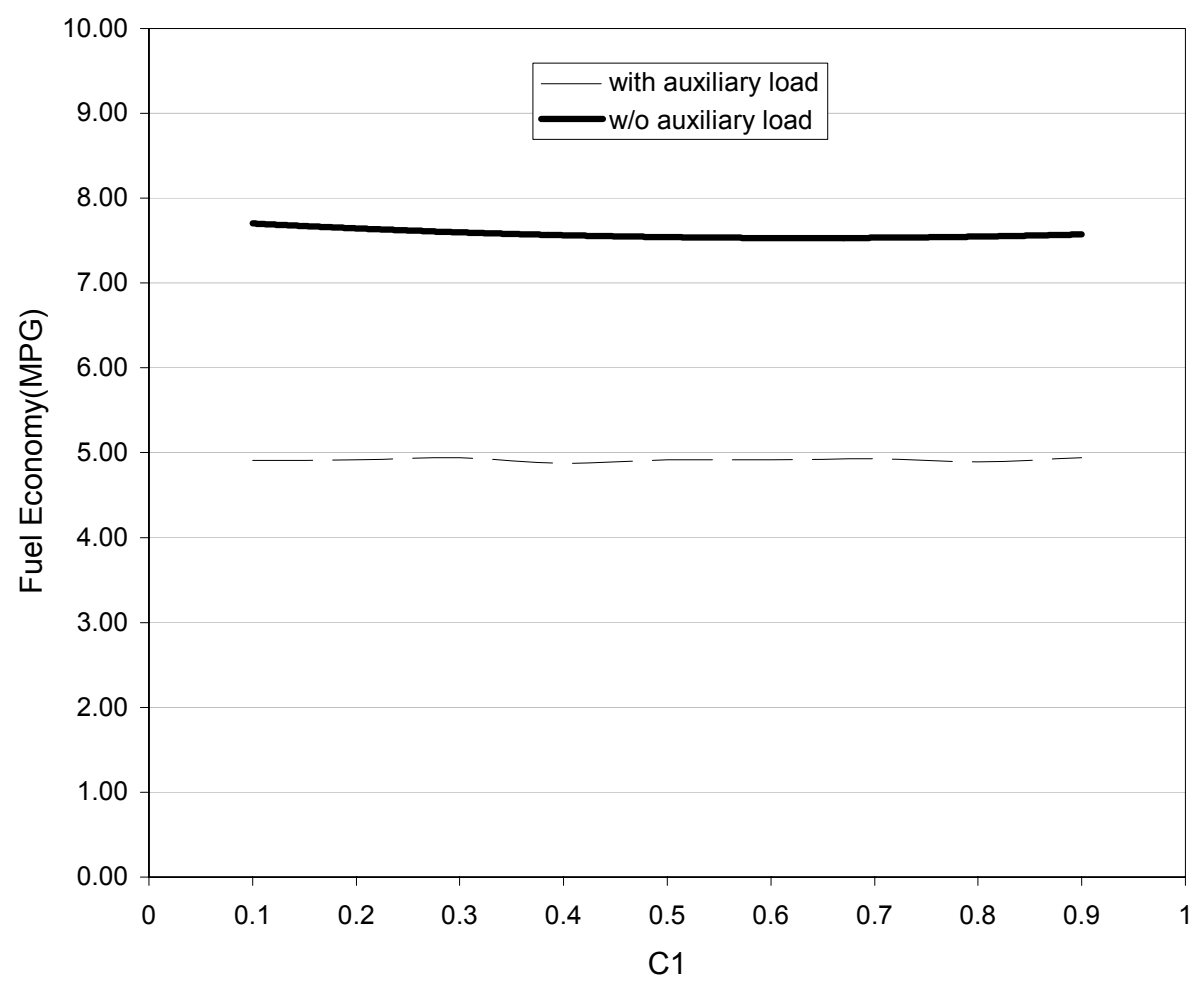

Figure 33: HEV Fuel Economy on CSHVC

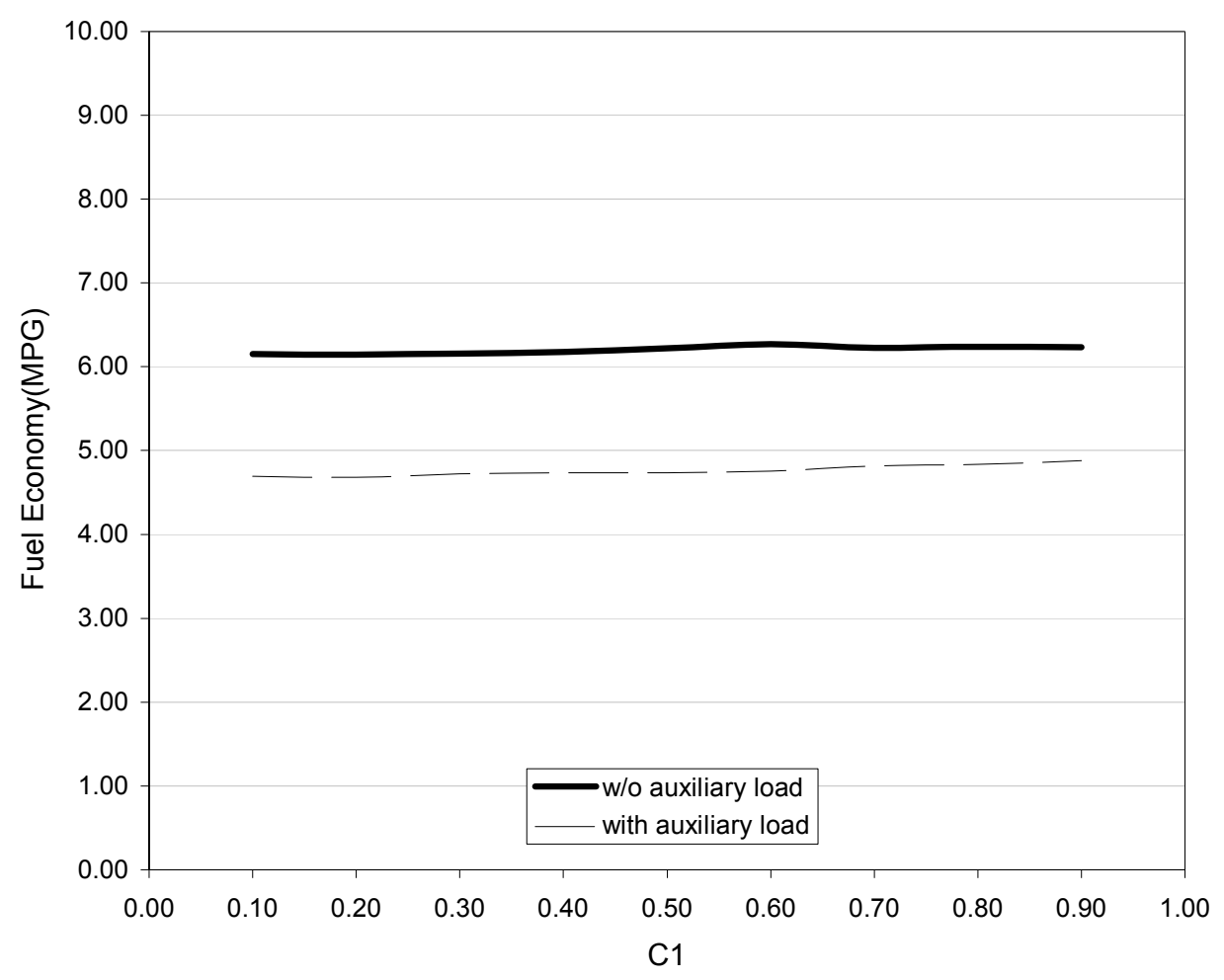

Figure 34: HEV Fuel Economy on UDDS cycle 


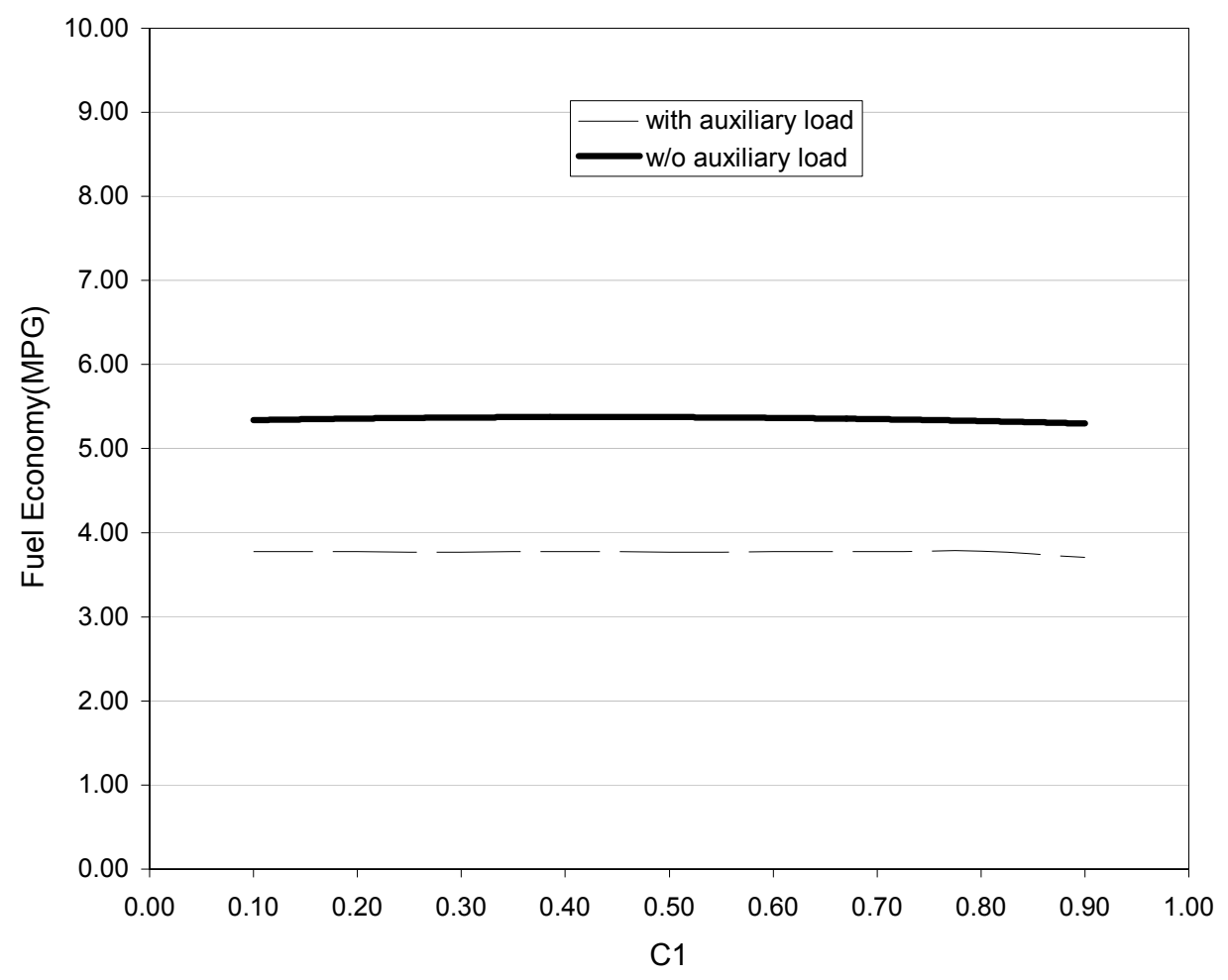

Figure 35: HEV Fuel Economy on UDDS1 cycle

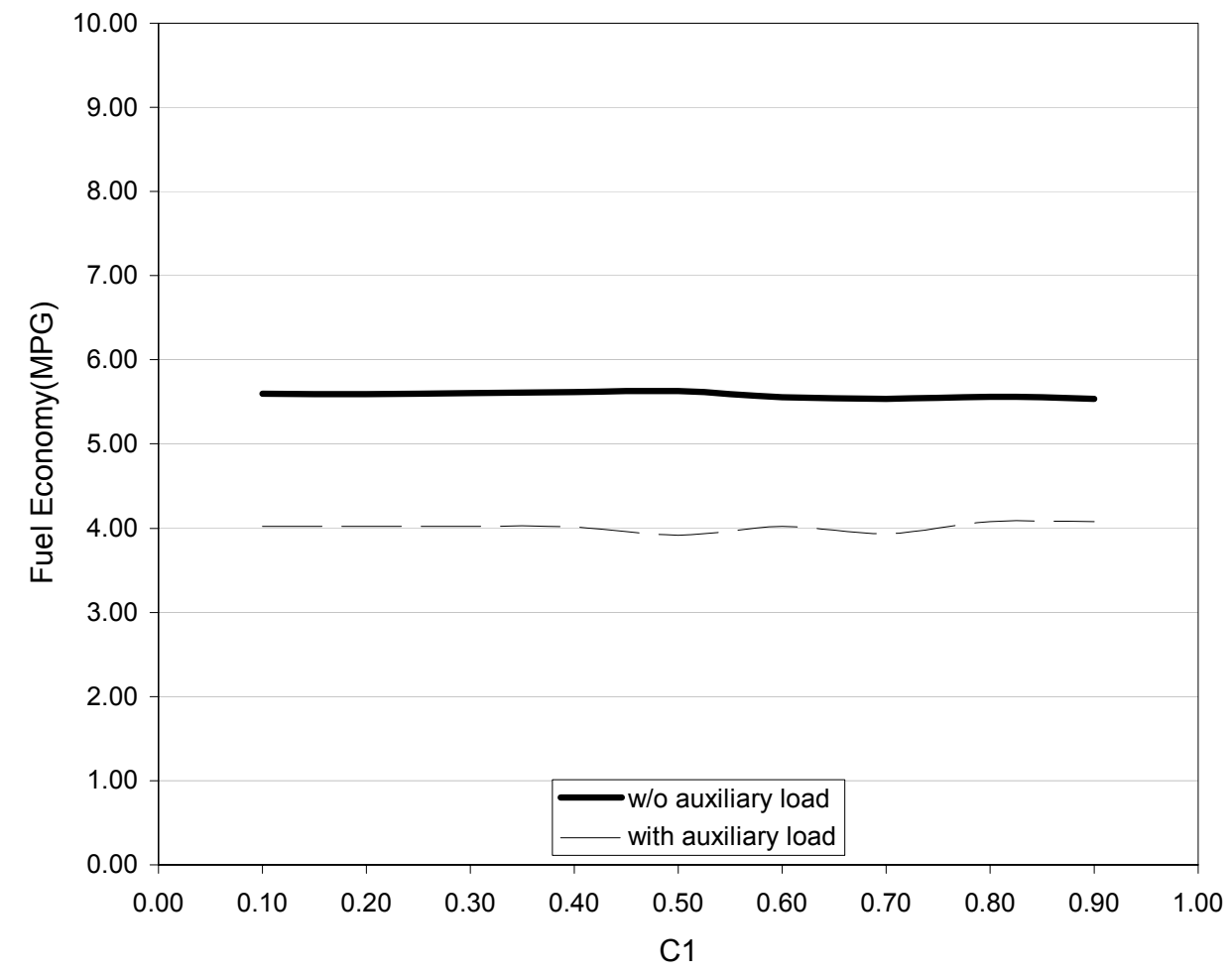

Figure 36: HEV Fuel Economy on UDDS2 cycle 
Also it is observed from the plots in Figures 31 through 36 that there is not much variation in the fuel economy over the range of 0.1 to $0.9 . \mathrm{C} 1$ being the percent of power required at wheels for a $\mathrm{HEV}$.

\subsection{State-of-Charge Correction}

For different values of $\mathrm{C} 1$ and $\mathrm{C} 2$ the fuel economy and change in state of charge correction are tabulated in Tables 8 through 19 over different driving cycles. The values of fuel economy were high for CBD cycle and CSHVC due error in simulations.

\begin{tabular}{|c|c|c|c|c|c|}
\hline & & \multicolumn{2}{|c|}{ w/o auxiliary load } & & \\
\hline C1 & $\mathrm{C} 2$ & SoC & delta SoC & MPG & \\
\hline 0.3 & $3.323 * 10-2$ & $60-60$ & -0.010 & 6.862 & \\
\hline 0.3 & $16.41 * 10-2$ & $90-90$ & -0.020 & 4.801 & \\
\hline 0.3 & $3.3^{*} 10-2$ & $60-90$ & 8745 & 5.694 & \\
\hline 0.3 & $17.41 * 10-2$ & $90-60$ & -8736 & 7.163 & \\
\hline \multicolumn{5}{|c|}{ Average of Fuel economy at $60 \%-60 \% \& 90 \%-90 \%$ SoC } & 5.831 \\
\hline \multicolumn{5}{|c|}{$Y$ intercept of $60 \%-90 \%$ and $90 \%-60 \%$ SoC } & 6.429 \\
\hline \multicolumn{5}{|l|}{$\%$ Error } & 9.295 \\
\hline C1 & $\mathrm{C} 2$ & SoC & delta SoC & MPG & \\
\hline 0.5 & $1.488 * 10-2$ & $60-60$ & 0.030 & 6.935 & \\
\hline 0.5 & $14.27^{*} 10-2$ & $90-90$ & 0.000 & 4.811 & \\
\hline 0.5 & $1.56 * 10-2$ & $60-90$ & 8749 & 4.989 & \\
\hline 0.5 & $15.372 * 10$ & $90-60$ & -8733 & 7.170 & \\
\hline \multicolumn{5}{|c|}{ Average of Fuel economy at $60 \%-60 \% \& 90 \%-90 \%$ SoC } & 5.873 \\
\hline \multicolumn{5}{|c|}{$Y$ intercept of $60 \%-90 \%$ and $90 \%-60 \%$ SoC } & 6.081 \\
\hline \multicolumn{5}{|l|}{$\%$ Error } & 3.411 \\
\hline
\end{tabular}

Table 8: SoC correction of HEV without auxiliary load on CBD cycle 


\begin{tabular}{|c|c|c|c|c|c|}
\hline & & \multicolumn{2}{|c|}{ with auxiliary load } & & \\
\hline C1 & C2 & SoC & delta SoC & MPG & \\
\hline 0.3 & $14.81^{* 10-2}$ & $60-60$ & 0.010 & 4.787 & \\
\hline 0.3 & $14.81^{*} 10-2$ & $90-90$ & 0.010 & 4.787 & \\
\hline$\overline{0.3}$ & $2.3^{*} 10-2$ & $60-90$ & 8035 & 4.220 & \\
\hline 0.3 & $16.37 * 10-2$ & $90-60$ & -8725 & 7.151 & \\
\hline \multicolumn{5}{|c|}{ Average of Fuel economy at $60 \%-60 \% \& 90 \%-90 \%$ SoC } & 4.787 \\
\hline \multicolumn{5}{|c|}{ Y intercept of $60 \%-90 \%$ and $90 \%-60 \%$ SoC } & 5.625 \\
\hline \multicolumn{5}{|l|}{$\%$ Error } & 14.90 \\
\hline C1 & $\mathrm{C} 2$ & SoC & delta SoC & MPG & \\
\hline 0.5 & $11.083^{*} 10$ & $60-60$ & -0.760 & 4.790 & \\
\hline 0.5 & $11.06^{*} 10-2$ & $90-90$ & -0.920 & 4.790 & \\
\hline 0.5 & $2 * 10-2$ & $60-90$ & 8270 & 4.225 & \\
\hline 0.5 & $12.966^{*} 10$ & $90-60$ & -8723 & 7.149 & \\
\hline \multicolumn{5}{|c|}{ Average of Fuel economy at $60 \%-60 \% \& 90 \%-90 \%$ SoC } & 4.790 \\
\hline \multirow{2}{*}{\multicolumn{5}{|c|}{\begin{tabular}{|l} 
Y intercept of $60 \%-90 \%$ and $90 \%-60 \%$ SoC \\
$\%$ Error
\end{tabular}}} & 5.648 \\
\hline & & & & & 15.19 \\
\hline
\end{tabular}

Table 9: SoC correction for HEV with auxiliary load over CBD cycle

\begin{tabular}{|c|c|c|c|c|c|}
\hline & & \multicolumn{2}{|c|}{ w/o auxiliary load } & & \\
\hline C1 & $\mathrm{C} 2$ & SoC & delta SoC & MPG & \\
\hline 0.3 & $1.963 * 10-2$ & $60-60$ & -0.152 & 7.397 & \\
\hline 0.3 & $1.963^{*} 10-2$ & $90-90$ & -0.152 & 7.397 & \\
\hline 0.3 & \multicolumn{2}{|c|}{$1.964 * 10-260-90$} & 8748 & 6.191 & \\
\hline 0.3 & \multicolumn{2}{|c|}{$1.964 * 10-290-60$} & -8748 & 9.577 & \\
\hline \multicolumn{5}{|c|}{ Average of Fuel economy at $60 \%-60 \% \& 90 \%-90 \%$ SoC } & 7.397 \\
\hline \multicolumn{4}{|c|}{$Y$ intercept of $60 \%-90 \%$ and $90 \%-60 \%$ SoC } & & 7.873 \\
\hline$\%$ Error & & & & & 6.040 \\
\hline $\mathrm{C} 1$ & $\mathrm{C} 2$ & SoC & delta SoC & MPG & \\
\hline 0.5 & $0.7366 * 10$ & $60-60$ & 0.119 & 7.419 & \\
\hline \multirow{2}{*}{$\frac{0.5}{0.5}$} & $0.7366 * 10$ & $90-90$ & 0.119 & 7.419 & \\
\hline & $0.759 * 10-2$ & $60-90$ & 8748 & 6.169 & \\
\hline 0.5 & $0.759 * 10-2$ & $90-60$ & -8656 & 9.558 & \\
\hline \multicolumn{5}{|c|}{ Average of Fuel economy at $60 \%-60 \% \& 90 \%-90 \%$ SoC } & 7.419 \\
\hline \multicolumn{4}{|c|}{$Y$ intercept of $60 \%-90 \%$ and $90 \%-60 \%$ SoC } & & 7.873 \\
\hline$\%$ Error & & & & & 5.761 \\
\hline
\end{tabular}

Table 10: SoC correction for HEV without auxiliary load over CSHVC 


\begin{tabular}{|c|c|c|c|c|c|}
\hline & & \multicolumn{2}{|c|}{ with auxiliary load } & & \\
\hline$\overline{C 1}$ & $\mathrm{C} 2$ & SoC & delta SoC & MPG & \\
\hline 0.3 & $100 * 10-2$ & $60-60$ & -10.50 & 4.939 & \\
\hline 0.3 & $75^{*} 10-2$ & $90-90$ & -13.99 & 4.924 & \\
\hline 0.3 & $2.9 * 10-2$ & $60-90$ & 8354 & 4.353 & \\
\hline 0.3 & $75^{*} 10-2$ & $90-60$ & -8762 & 5.552 & \\
\hline \multicolumn{5}{|c|}{ Average of Fuel economy at $60 \%-60 \% \& 90 \%-90 \%$ SoC } & 4.932 \\
\hline \multicolumn{5}{|c|}{ Y intercept of $60 \%-90 \%$ and $90 \%-60 \%$ SoC } & 4.938 \\
\hline \multicolumn{5}{|l|}{$\%$ Error } & 0.136 \\
\hline $\mathrm{C} 1$ & $\mathrm{C} 2$ & SoC & delta SoC & MPG & \\
\hline 0.5 & $106 * 10-2$ & $60-60$ & -7.513 & 4.938 & \\
\hline 0.5 & $96 * 10-2$ & $90-90$ & -7.790 & 4.934 & \\
\hline 0.5 & $2.9 * 10-2$ & $60-90$ & 8493 & 4.381 & \\
\hline 0.5 & $6 * 10-2$ & $90-60$ & $\overline{-8870}$ & 5.423 & \\
\hline \multicolumn{5}{|c|}{ Average of Fuel economy at $60 \%-60 \% \& 90 \%-90 \%$ SoC } & 4.936 \\
\hline \multicolumn{5}{|c|}{$\mathrm{Y}$ intercept of $60 \%-90 \%$ and $90 \%-60 \%$ SoC } & 4.891 \\
\hline \multicolumn{5}{|l|}{$\%$ Error } & -0.926 \\
\hline
\end{tabular}

Table 11: SoC correction for HEV with auxiliary load over CSHVC

\begin{tabular}{|c|c|c|c|c|c|}
\hline \multirow[b]{2}{*}{ C1 } & \multirow[b]{2}{*}{$\mathrm{C} 2$} & \multicolumn{2}{|c|}{ w/o auxiliary load } & & \\
\hline & & SoC & delta SoC & MPG & \\
\hline 0.3 & $3.024 * 10-2$ & $60-60$ & 0.051 & 5.256 & \\
\hline 0.3 & $3.024 * 10-2$ & $90-90$ & 0.051 & 5.256 & \\
\hline 0.3 & $3.024 * 10-2$ & $60-90$ & 8748 & 3.435 & \\
\hline$\overline{0.3}$ & $4 * 10-2$ & $90-60$ & -8168 & 6.365 & \\
\hline \multicolumn{5}{|c|}{ Average of Fuel economy at $60 \%-60 \% \& 90 \%-90 \%$ SoC } & 5.256 \\
\hline \multicolumn{4}{|c|}{$Y$ intercept of $60 \%-90 \%$ and $90 \%-60 \%$ SoC } & & 4.950 \\
\hline \multirow{2}{*}{$\begin{array}{l}\% \text { Error } \\
\mathrm{C} 1\end{array}$} & & & & & -6.175 \\
\hline & $\mathrm{C} 2$ & SoC & delta SoC & MPG & \\
\hline 0.4 & $1.525^{*} 10-2$ & $60-60$ & -0.043 & 5.262 & \\
\hline \multirow{2}{*}{0.4} & $1.525^{*} 10-2$ & $90-90$ & -0.043 & 5.262 & \\
\hline & $1.55 * 10-2$ & $60-90$ & 8742 & 3.395 & \\
\hline 0.4 & $5 * 10-2$ & $90-60$ & -8110 & 6.365 & \\
\hline \multicolumn{5}{|c|}{ Average of Fuel economy at $60 \%-60 \% \& 90 \%-90 \%$ SoC } & 5.262 \\
\hline \multicolumn{4}{|c|}{ Y intercept of $60 \%-90 \%$ and $90 \%-60 \%$ SoC } & & 4.936 \\
\hline$\%$ Error & & & & & -6.611 \\
\hline
\end{tabular}

Table 12: SoC correction for HEV without auxiliary load over Manhattan cycle 


\begin{tabular}{|c|c|c|c|c|c|}
\hline & & \multicolumn{2}{|c|}{ with auxiliary load } & & \\
\hline C1 & C2 & SoC & delta SoC & MPG & \\
\hline 0.3 & $86.8^{*} 10-2$ & $60-60$ & 0.045 & 2.680 & \\
\hline 0.3 & $86.6 * 10-2$ & $90-90$ & -0.064 & 2.680 & \\
\hline 0.3 & $3.9 * 10-2$ & $60-90$ & 8418 & 2.183 & \\
\hline 0.3 & $9 * 10-2$ & $90-60$ & -8858 & 3.543 & \\
\hline \multicolumn{5}{|c|}{ Average of Fuel economy at $60 \%-60 \% \& 90 \%-90 \%$ SoC } & 2.680 \\
\hline \multicolumn{4}{|c|}{$\mathrm{Y}$ intercept of $60 \%-90 \%$ and $90 \%-60 \%$ SoC } & & 2.846 \\
\hline \multirow{2}{*}{\begin{tabular}{|l}
$\%$ Error \\
C1 \\
\end{tabular}} & & & & & 5.826 \\
\hline & $\mathrm{C} 2$ & SoC & delta SoC & MPG & \\
\hline \multirow{2}{*}{$\frac{0.4}{0.4}$} & $72.7 * 10-2$ & $60-60$ & 0.016 & 2.675 & \\
\hline & $72.67^{* 10-2}$ & $90-90$ & -0.002 & 2.675 & \\
\hline \multirow{2}{*}{$\frac{0.4}{0.4}$} & $3 * 10-2$ & $60-90$ & 8369 & 2.170 & \\
\hline & $8.3^{*} 10-2$ & $90-60$ & -8846 & 3.546 & \\
\hline \multicolumn{5}{|c|}{ Average of Fuel economy at $60 \%-60 \% \& 90 \%-90 \%$ SoC } & 2.675 \\
\hline \multicolumn{4}{|c|}{ Y intercept of $60 \%-90 \%$ and $90 \%-60 \%$ SoC } & & 2.839 \\
\hline$\%$ Error & & & & & 5.777 \\
\hline
\end{tabular}

Table 13: SoC correction of HEV with auxiliary load over Manhattan cycle

\begin{tabular}{|c|c|c|c|c|c|}
\hline & \multirow[b]{2}{*}{$\mathrm{C} 2$} & \multicolumn{2}{|c|}{ w/o auxiliary load } & \multirow[b]{2}{*}{ MPG } & \\
\hline C1 & & SoC & delta SoC & & \\
\hline 0.4 & $1.603^{*} 10-2$ & $60-60$ & 0.153 & 6.015 & \\
\hline 0.4 & $1.603^{*} 10-2$ & $90-90$ & 0.153 & 6.015 & \\
\hline 0.4 & $1.6^{*} 10-2$ & $60-90$ & 8742 & 5.170 & \\
\hline 0.4 & $1.6 * 10-2$ & $90-60$ & -8727 & 7.514 & \\
\hline \multicolumn{5}{|c|}{ Average of Fuel economy at $60 \%-60 \% \& 90 \%-90 \%$ SoC } & 6.015 \\
\hline \multicolumn{4}{|c|}{$Y$ intercept of $60 \%-90 \%$ and $90 \%-60 \%$ SoC } & & 4.732 \\
\hline$\%$ Error & & & & & -27.11 \\
\hline $\mathrm{C} 1$ & $\mathrm{C} 2$ & SoC & delta SoC & MPG & \\
\hline 0.6 & $0.71 * 10-2$ & $60-60$ & -0.293 & 6.121 & \\
\hline 0.6 & $0.71^{*} 10-2$ & $90-90$ & -0.293 & 6.121 & \\
\hline$\overline{0.6}$ & $0.78 * 10-2$ & $60-90$ & 8762 & 5.228 & \\
\hline 0.6 & $0.46 * 10-2$ & $90-60$ & -8420 & 7.607 & \\
\hline \multicolumn{5}{|c|}{ Average of Fuel economy at $60 \%-60 \% \& 90 \%-90 \%$ SoC } & 6.121 \\
\hline \multicolumn{4}{|c|}{$Y$ intercept of $60 \%-90 \%$ and $90 \%-60 \%$ SoC } & & 4.758 \\
\hline$\%$ Error & & & & & -28.65 \\
\hline
\end{tabular}

Table 14: SoC correction for HEV without auxiliary load over UDDS cycle 


\begin{tabular}{|c|c|c|c|c|c|}
\hline & & \multicolumn{2}{|c|}{ with auxiliary laod } & & \\
\hline C1 & C2 & SoC & delta SoC & MPG & \\
\hline 0.4 & $75^{*} 10-2$ & $60-60$ & -12.00 & 4.732 & \\
\hline 0.4 & $75 * 10-2$ & $90-90$ & -12.00 & 4.732 & \\
\hline 0.4 & $3.5^{*} 10-2$ & $60-90$ & 8478 & 4.246 & \\
\hline 0.4 & $5 * 10-2$ & $90-60$ & -8933 & 5.244 & \\
\hline \multicolumn{5}{|c|}{ Average of Fuel economy at $60 \%-60 \% \& 90 \%-90 \%$ SoC } & 4.732 \\
\hline \multicolumn{4}{|c|}{$\mathrm{Y}$ intercept of $60 \%-90 \%$ and $90 \%-60 \%$ SoC } & & 6.343 \\
\hline & & & & & 25.40 \\
\hline $\begin{array}{l}\% \text { Error } \\
\text { C1 }\end{array}$ & $\mathrm{C} 2$ & SoC & delta SoC & MPG & \\
\hline 0.8 & $2.76^{*} 10-2$ & $60-60$ & -0.223 & 4.757 & \\
\hline 0.8 & $58 * 10-2$ & $90-90$ & -5.170 & 4.829 & \\
\hline 0.8 & $2.5^{*} 10-2$ & $60-90$ & 8753 & 4.370 & \\
\hline 0.8 & $11 * 10-2$ & $90-60$ & -8773 & 5.381 & \\
\hline \multicolumn{5}{|c|}{ Average of Fuel economy at $60 \%-60 \% \& 90 \%-90 \%$ SoC } & 4.793 \\
\hline \multicolumn{4}{|c|}{ Y intercept of $60 \%-90 \%$ and $90 \%-60 \%$ SoC } & & 6.341 \\
\hline$\%$ Error & & & & & 24.42 \\
\hline
\end{tabular}

Table 15: SoC correction for HEV with auxiliary load over UDDS cycle

\begin{tabular}{|c|c|c|c|c|c|}
\hline & \multirow[b]{2}{*}{ C2 } & \multicolumn{2}{|c|}{ without auxiliary load } & & \\
\hline $\mathrm{C} 1$ & & \multirow{2}{*}{ SoC } & \multirow{2}{*}{\begin{tabular}{|r|} 
delta SoC \\
-0.492
\end{tabular}} & MPG & \\
\hline 0.2 & $2.539 * 10-2$ & & & 5.244 & \\
\hline$\overline{0.3}$ & $2.539 * 10-2$ & $90-90$ & -0.492 & 5.244 & \\
\hline 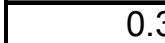 & $2.539 * 10-2$ & $60-90$ & 8747 & 4.284 & \\
\hline$\overline{0.3}$ & $2.539 * 10-2$ & $90-60$ & -8745 & 5.832 & \\
\hline \multicolumn{5}{|c|}{ Average of Fuel economy at $60 \%-60 \% \& 90 \%-90 \%$ SoC } & $\overline{5.244}$ \\
\hline \multicolumn{4}{|c|}{$\mathrm{Y}$ intercept of $60 \%-90 \%$ and $90 \%-60 \%$ SoC } & & 5.058 \\
\hline$\%$ Error & & & & & -3.675 \\
\hline $\mathrm{C} 1$ & $\mathrm{C} 2$ & SoC & delta SoC & MPG & \\
\hline 0.4 & $1.777^{*} 10-2$ & $60-60$ & -0.734 & 5.249 & \\
\hline 0.4 & $2.168 * 10-2$ & $90-90$ & 0.284 & 5.249 & \\
\hline$\overline{0.4}$ & $2.16 * 10-2$ & $60-90$ & 8736 & 4.286 & \\
\hline 0.4 & $2.168 * 10-2$ & $90-60$ & -8738 & 6.833 & \\
\hline \multicolumn{5}{|c|}{ Average of Fuel economy at $60 \%-60 \% \& 90 \%-90 \%$ SoC } & 5.249 \\
\hline \multicolumn{4}{|c|}{ Y intercept of $60 \%-90 \%$ and $90 \%-60 \%$ SoC } & & 5.559 \\
\hline$\%$ Error & & & & & 5.583 \\
\hline
\end{tabular}

Table 16: SoC correction for HEV without auxiliary load over UDDS1 cycle 


\begin{tabular}{|c|c|c|c|c|c|}
\hline & & \multicolumn{2}{|c|}{ with auxiliary load } & & \\
\hline C1 & $\mathrm{C} 2$ & SoC & delta SoC & MPG & \\
\hline 0.3 & $3.415^{\star} 10-2$ & $60-60$ & 0.493 & 3.722 & \\
\hline 0.3 & $3.41 * 10-2$ & $90-90$ & -0.761 & 3.722 & \\
\hline 0.3 & $3.45^{*} 10-2$ & $60-90$ & 8774 & 3.234 & \\
\hline 0.3 & $3.53 * 10-2$ & $90-60$ & -8657 & 4.394 & \\
\hline \multicolumn{5}{|c|}{ Average of Fuel economy at $60 \%-60 \% \& 90 \%-90 \%$ SoC } & 3.722 \\
\hline \multicolumn{4}{|c|}{$Y$ intercept of $60 \%-90 \%$ and $90 \%-60 \%$ SoC } & & 3.818 \\
\hline$\%$ Error & & & & & 2.512 \\
\hline C1 & $\mathrm{C} 2$ & SoC & delta SoC & MPG & \\
\hline $\begin{array}{r}0.4 \\
\end{array}$ & $2.933^{*} 10-2$ & $60-60$ & -0.986 & 3.723 & \\
\hline \multirow{2}{*}{$\frac{0.4}{0.4}$} & $2.932 * 10-2$ & $90-90$ & -0.736 & 3.723 & \\
\hline & $2.933^{*} 10-2$ & $60-90$ & 8747 & 3.236 & \\
\hline$\overline{0.4}$ & $2.932 * 10-2$ & $90-60$ & -8749 & 4.403 & \\
\hline \multicolumn{5}{|c|}{ Average of Fuel economy at $60 \%-60 \% \& 90 \%-90 \%$ SoC } & 3.723 \\
\hline \multicolumn{4}{|c|}{$Y$ intercept of $60 \%-90 \%$ and $90 \%-60 \%$ SoC } & & 3.819 \\
\hline$\%$ Error & & & & & 2.524 \\
\hline
\end{tabular}

Table 17: SoC correction for HEV with auxiliary load over UDDS1 cycle

\begin{tabular}{|c|c|c|c|c|c|}
\hline & \multirow[b]{2}{*}{$\mathrm{C} 2$} & \multicolumn{2}{|c|}{ w/o auxilary load } & \multirow[b]{2}{*}{ MPG } & \\
\hline C1 & & SoC & delta SoC & & \\
\hline 0.5 & $0.751 * 10-2$ & $60-60$ & 0.207 & 5.471 & \\
\hline 0.5 & $0.751 * 10-2$ & $90-90$ & 0.207 & 5.471 & \\
\hline 0.5 & $0.793 * 10-2$ & $60-90$ & 8716 & 4.515 & \\
\hline 0.5 & $0.621 * 10-2$ & $90-60$ & -8688 & 7.061 & \\
\hline \multicolumn{5}{|c|}{ Average of Fuel economy at $60 \%-60 \% \& 90 \%-90 \%$ SoC } & $\overline{5.471}$ \\
\hline \multicolumn{4}{|c|}{$Y$ intercept of $60 \%-90 \%$ and $90 \%-60 \%$ SoC } & & 5.790 \\
\hline & & & & & 5.509 \\
\hline \begin{tabular}{|l}
$\%$ Error \\
$\mathrm{C} 1$ \\
\end{tabular} & $\mathrm{C} 2$ & SoC & delta SoC & MPG & \\
\hline 0.7 & $0.001 * 10-2$ & $60-60$ & 25.72 & 5.501 & \\
\hline 0.7 & $0.001 * 10-2$ & $90-90$ & 25.72 & 5.501 & \\
\hline 0.7 & $0.31 * 10-2$ & $60-90$ & 8748 & 4.526 & \\
\hline 0.7 & $16 * 10-2$ & $90-60$ & -7004 & 6.622 & \\
\hline \multicolumn{5}{|c|}{ Average of Fuel economy at $60 \%-60 \% \& 90 \%-90 \%$ SoC } & 5.501 \\
\hline \multicolumn{4}{|c|}{ Y intercept of $60 \%-90 \%$ and $90 \%-60 \%$ SoC } & & 5.690 \\
\hline$\%$ Error & & & & & 3.322 \\
\hline
\end{tabular}

Table 18: SoC correction for HEV without auxiliary load over UDDS2 cycle 


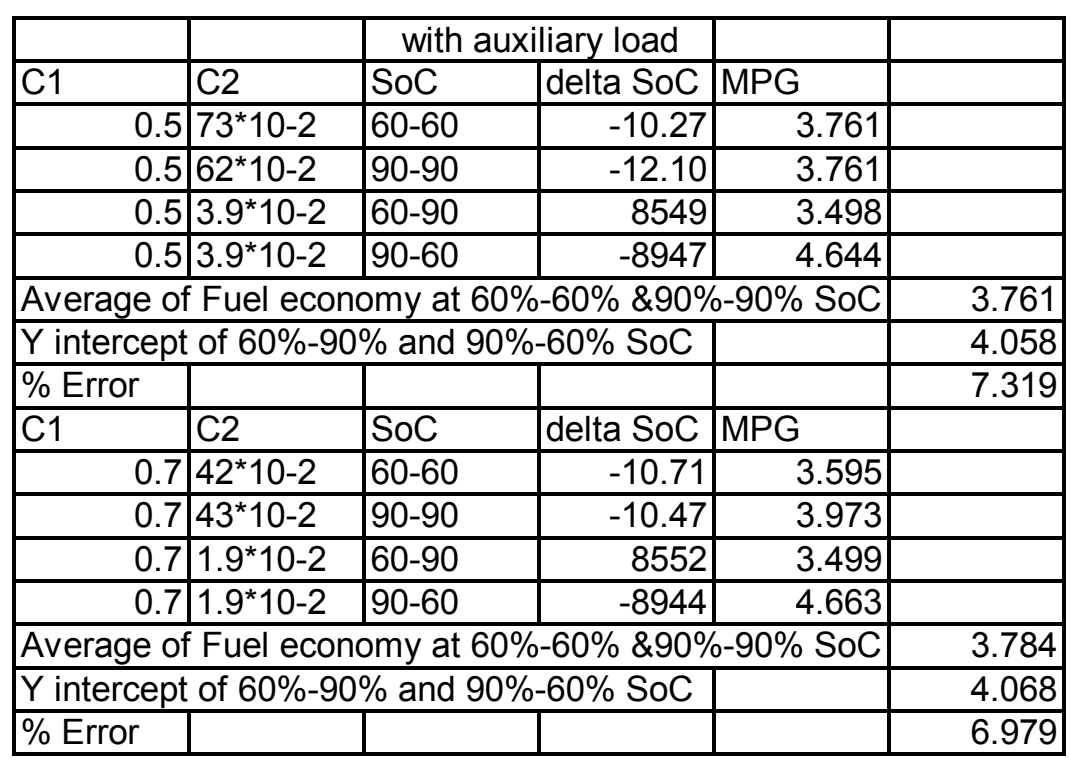

Table 19: SoC correction for HEV with auxiliary load over UDDS2 cycle

The variation in fuel economy is due to the way the control strategy forces the vehicle to maintain charge-sustaining operation. In the UDDS1 Cycle, the high power requirement segments are near the end of the cycle forcing the engine to follow the road load closely throughout the cycle to avoid depleting the batteries. In the UDDS2 cycle, the presence of the high power events near the beginning of the cycle allows the vehicle to recover SoC during the less demanding end portions through charging while driving. This phenomenon is not entirely realistic since requiring an HEV to return to the initial SoC after each use is not always possible.

Two terms associated with hybrid-electric buses are charge-sustaining and charge-depleting. The former implies that the vehicle derives all of its fuel from the APU, while the latter implies that the vehicle must eventually be recharged via the electric utility grid. In an ideal world when the bus finishes an emission test cycle, the batteries would have the same SOC at the end of the test as at the beginning (a net SOC difference of zero). This would allow the data to be used without correcting for state of charge. This 
is currently how SAE J1711 (a SAE recommended practice for light-duty hybrid test procedures) covers SOC corrections by limiting the SOC differential to 1 percent of the energy expended during the cycle [4].

The net change in SOC, in Watt-hr was provided at the end of each test. If the net change in battery SOC was not zero during the test, a correction was necessary. After the SOC information was plotted against fuel economy and emissions data, a linear interpolation, or in some cases extrapolation, was performed to establish what the fuel economy or emissions would be at a net change in SOC of zero. In other words, the data was corrected to a net zero change in SOC. In addition, an average (consistent with SAE J1711) was performed for data points where the net change in energy during a test was not equal to zero (nearly zero). Thus the SoC corrected fuel economy is used for comparison with the in-use vehicles.

Tables 8 to 19 show the SoC correction for different cycles. Figures 37 to 48 show $\mathrm{SoC}$ correction for different driving cycles. Here the $\mathrm{SoC}$ is shown for only few values of $\mathrm{C} 1$. For more values of $\mathrm{SoC}$ for different values of $\mathrm{C} 1$ see appendix. Figures 37 to 48 the fuel economy vs change in SoC (Delta SoC) were plotted. In these Figures the fuel economy for SoC starting at $60 \%$ and ending at $60 \%(60 \%-60 \%)$, SoC starting at $90 \%$ and ending at $90 \%(90 \%-90 \%)$ over lap on one another. So only 3 points are visible instead of 4 in these graphs. When initial SoC (at the beginning of the simulation) is different then it implies that there are different amounts of power in and out of batteries. Depending on the charge in the battery the SoC can be high or low. The average of the fuel economy for SoC $60 \%-60 \%$ and $90 \%-90 \%$ were taken and compared with the intercept of the linear curve fitted to the SoC starting at $60 \%$ and ending at $90 \%(60 \%$ - 
$90 \%)$ and SoC starting at $90 \%$ and ending at $60 \%(90 \%-60 \%)$. The difference in fuel economy for these is the error. The smaller the error the more accurate is the simulation. The percent differences between the zero delta SoC points (SoC 60\%-60\% \& SoC 90\%$90 \%$ ) and line intersecting the $\mathrm{SoC}$ at $60 \%$ and $\mathrm{SoC}$ at $90 \%$ have been tabulated in Tables 8 through 19 and in Tables 25 through 36 in appendix.

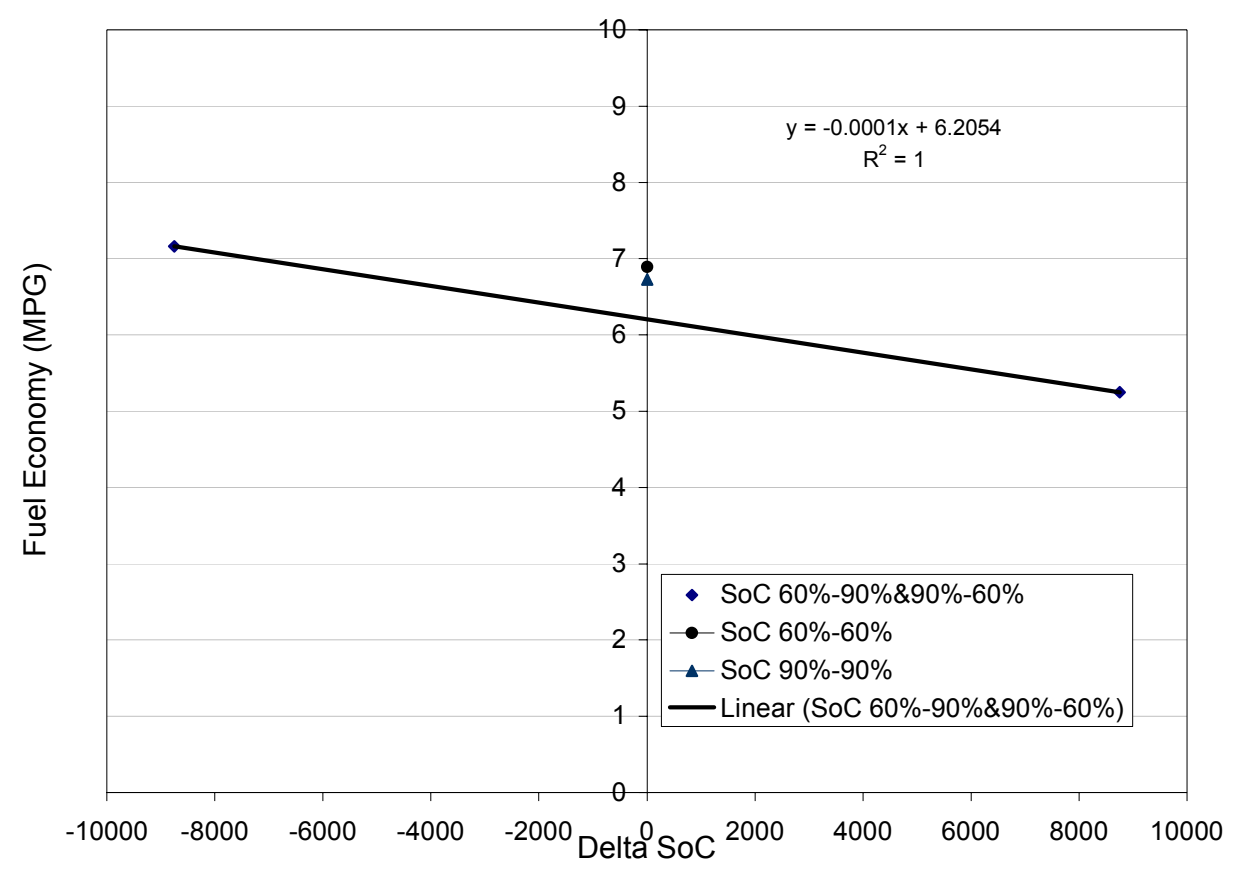

Figure 37: MPG of HEV without auxiliary load on CBD cycle at $\mathrm{C1}=0.4$ 


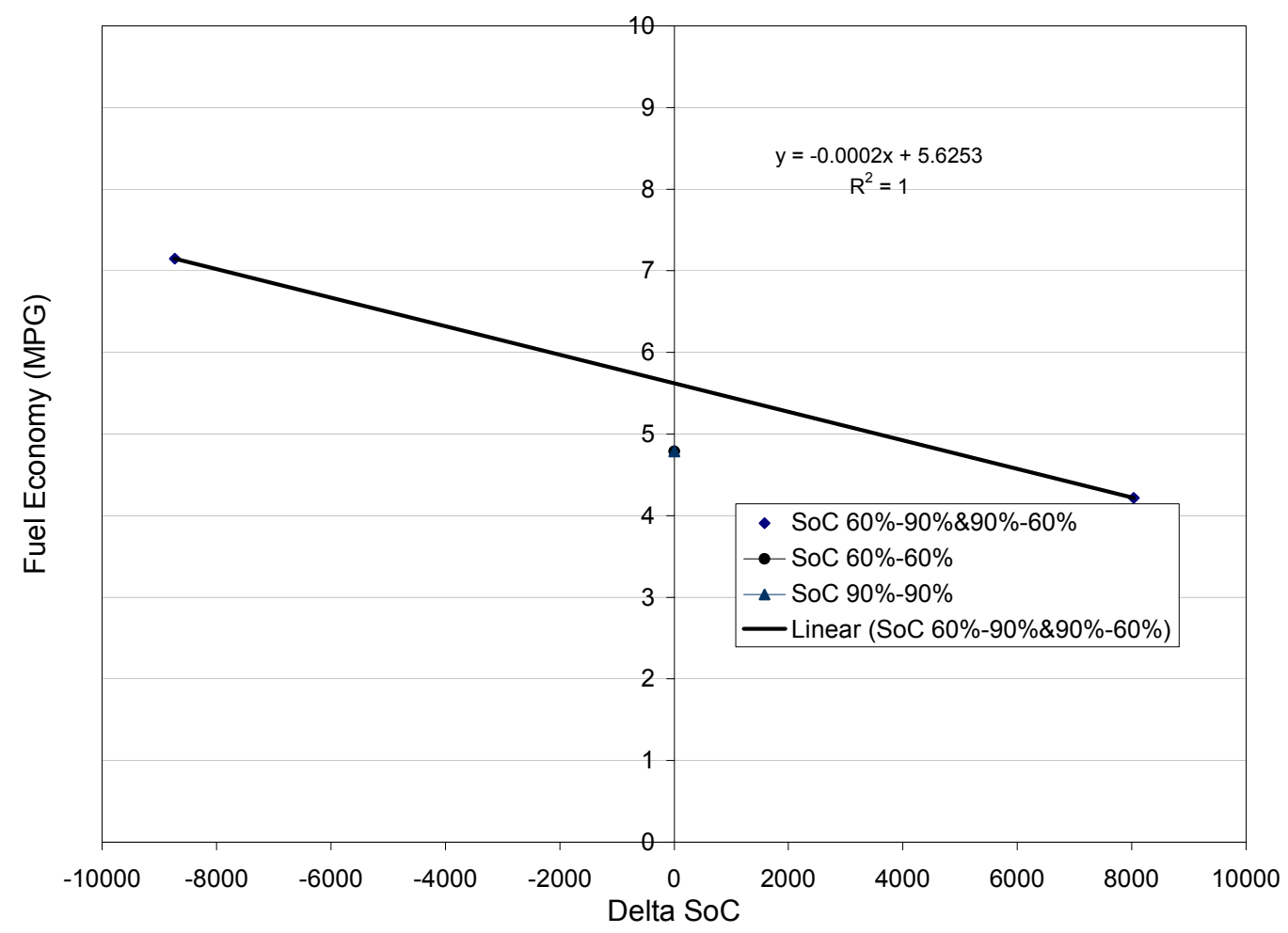

Figure 38: The points representing MPG at SoC 60\%-60\% and SoC 90\%-90\% overlap for a HEV over $\mathrm{CBD}$ cycle at $\mathrm{C} 1=0.3$ 


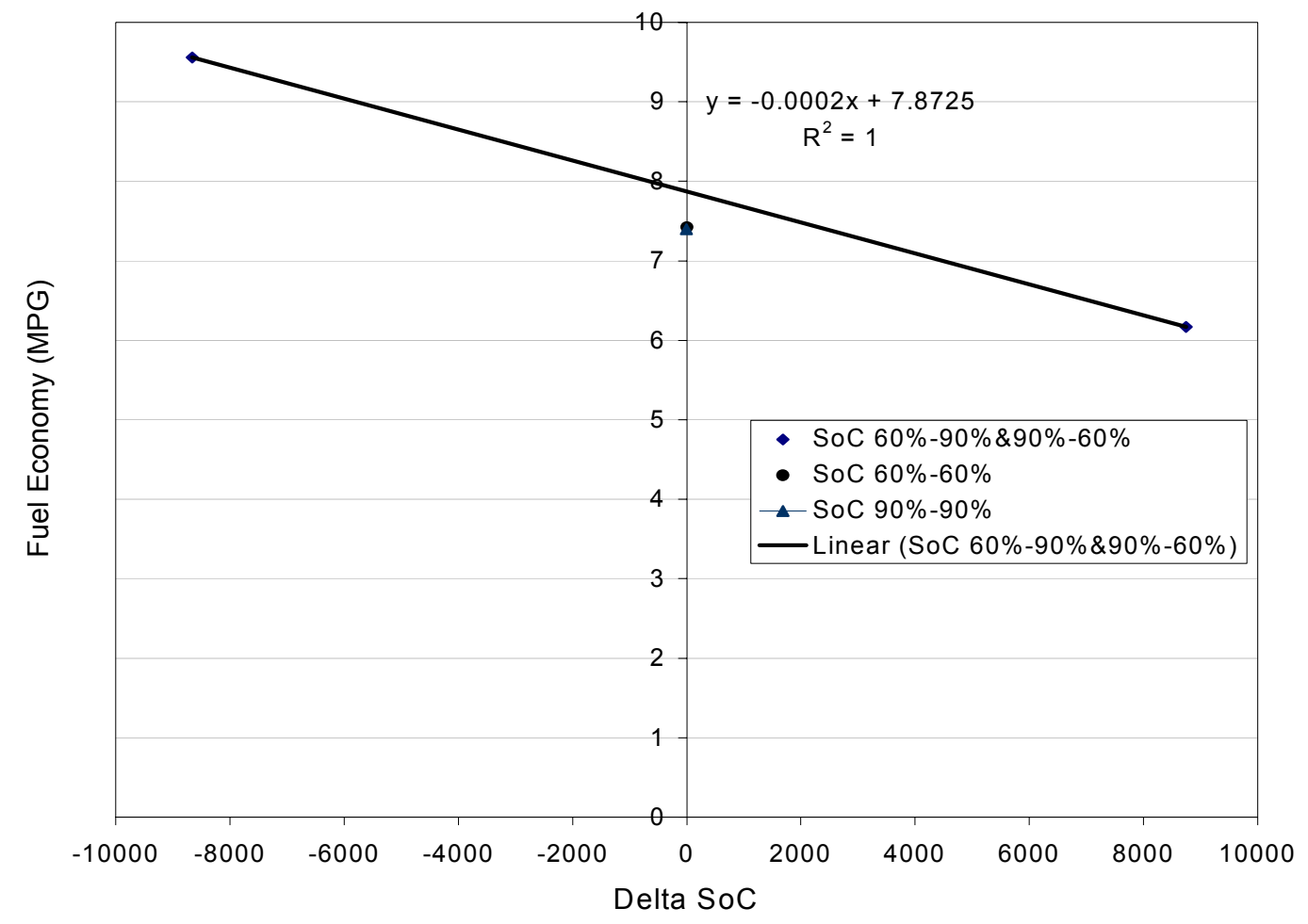

Figure 39: 6\% difference is observed for the points representing MPG of HEV at zero delta SoC and line intersecting SoC at $60 \%$ and at $90 \%$ over CSHVC at $\mathrm{C1}=0.3$ 


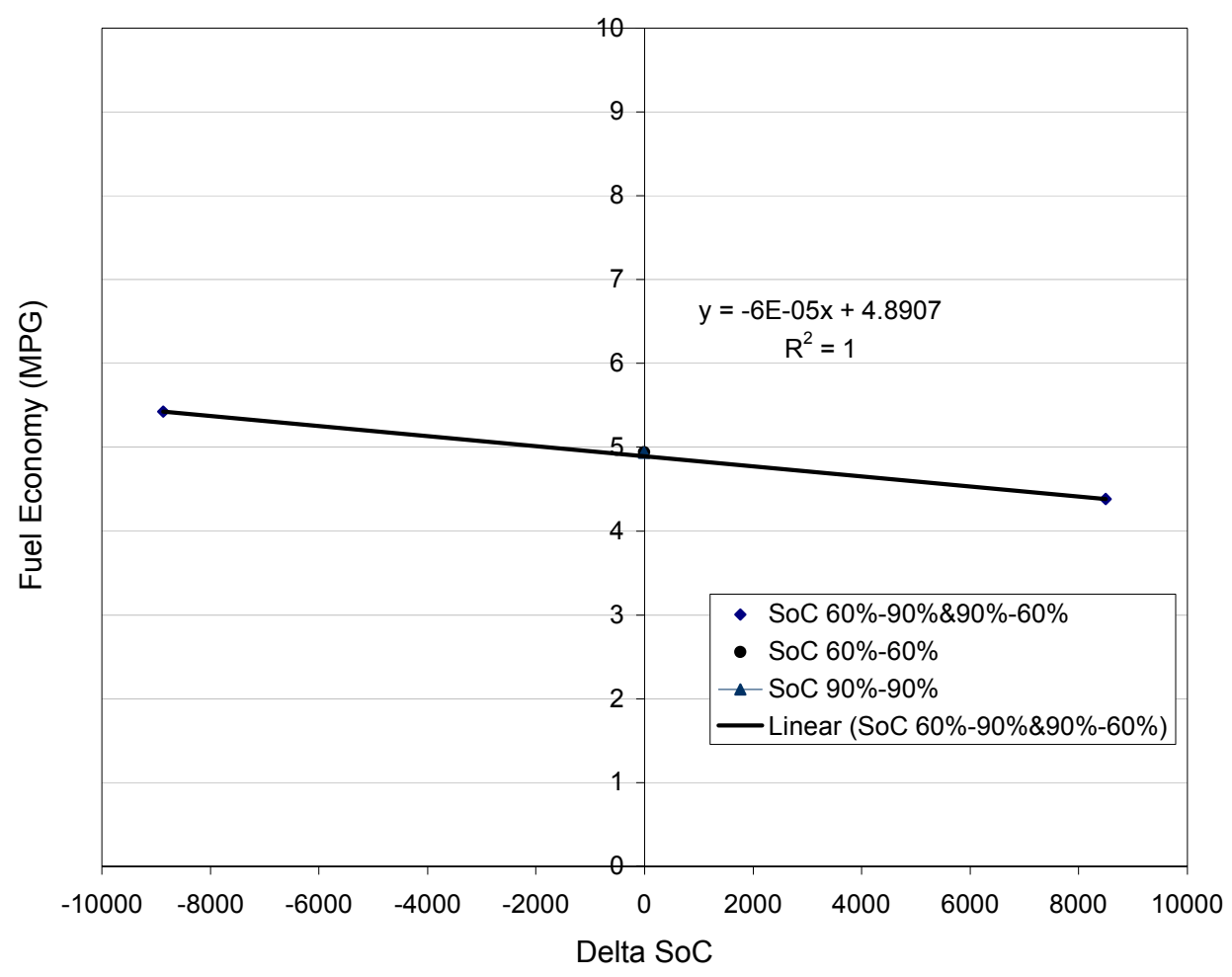

Figure 40:-0.9\% difference is observed for the points representing MPG of HEV at zero delta SoC and line intersecting $\mathrm{SoC}$ at $60 \%$ \& at $90 \%$ over CSHVC at $\mathrm{C} 1=0.5$ 


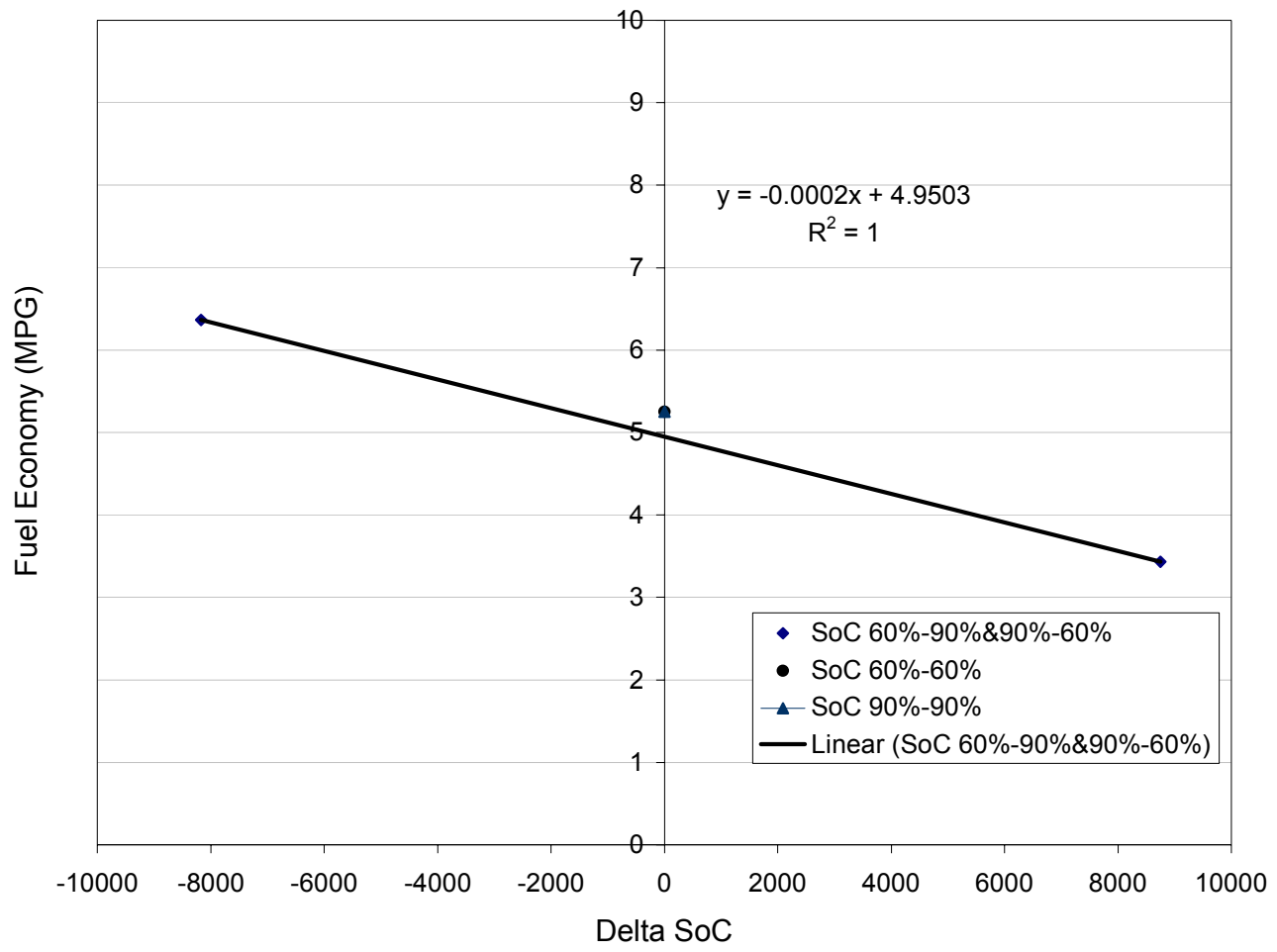

Figure 41:-6\% difference is observed between the points representing MPG of HEV at zero delta SoC and line intersecting $\mathrm{SoC}$ at $60 \% \&$ at $90 \%$ over CSHVC at $\mathrm{C} 1=0.5$ 


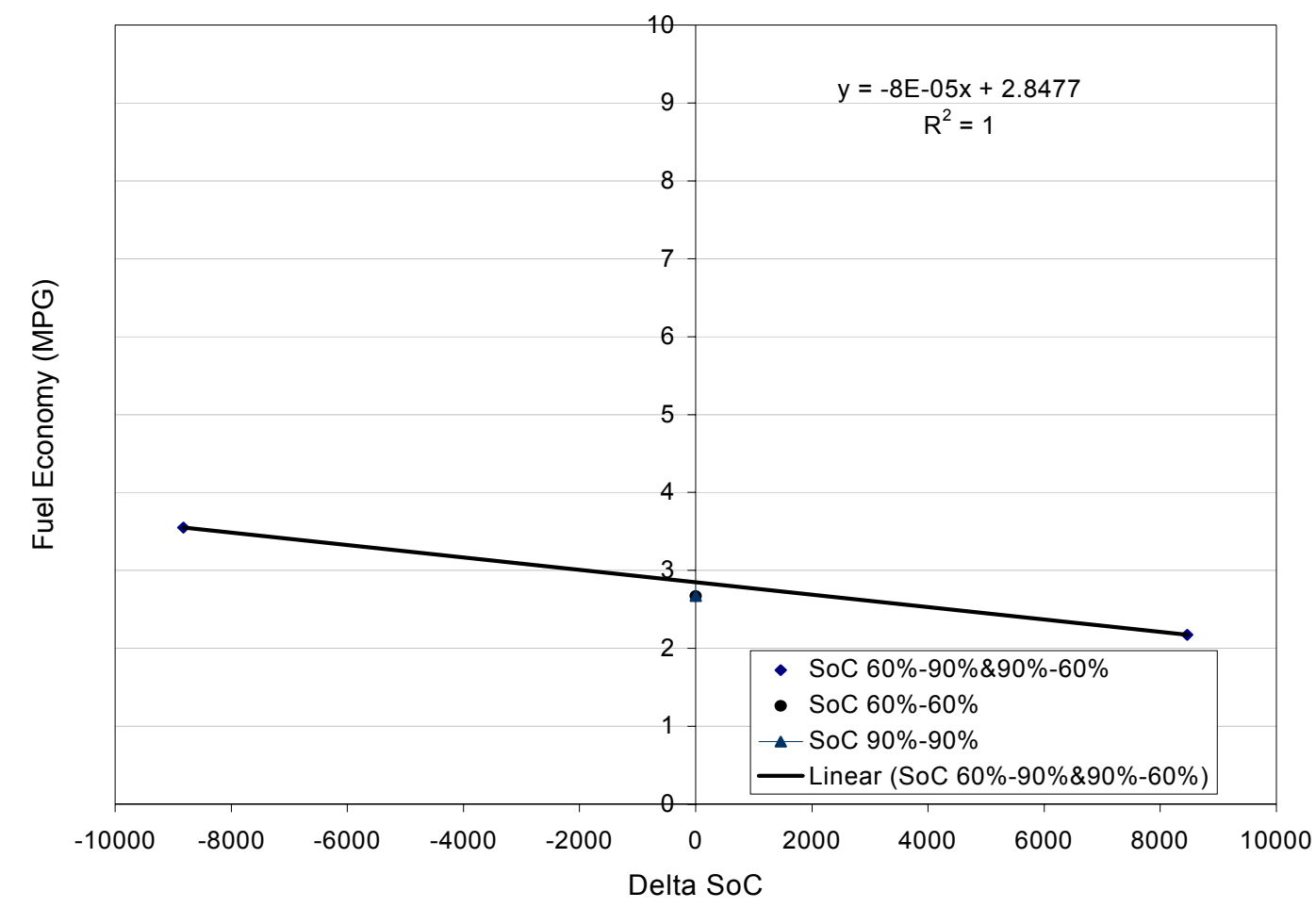

Figure 42: MPG of HEV with auxiliary load on Manhattan Cycle at $\mathrm{C} 1=0.5$, with a difference of $6 \%$ between the MPG of zero delta SoC points and line intersecting SoC at $60 \% \& \mathrm{SoC}$ at $90 \%$ 


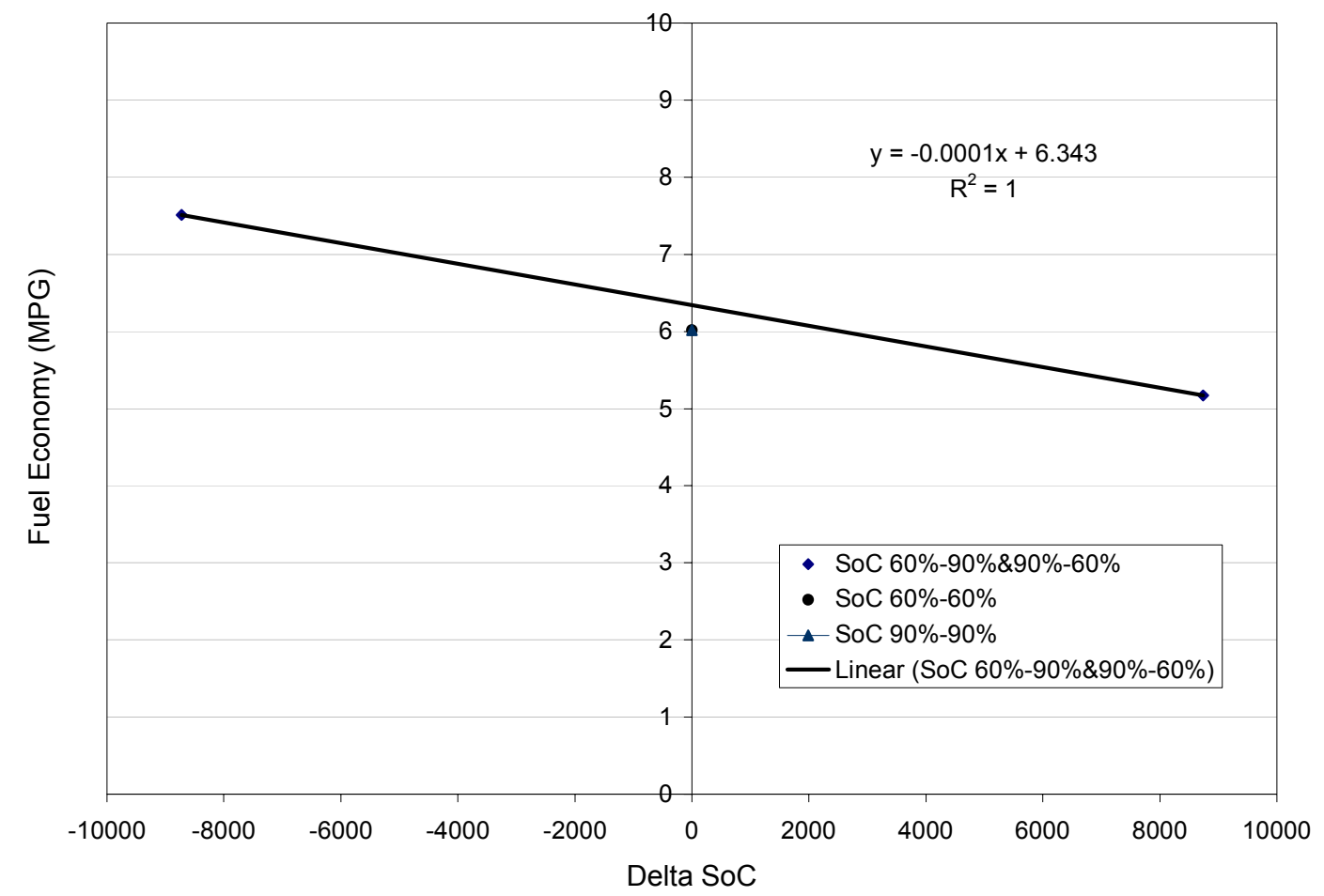

Figure 43: MPG of HEV without auxiliary load on UDDS cycle at $\mathrm{C1}=\mathbf{0 . 4}$, with a difference of $\mathbf{- 2 7 \%}$ between the MPG of zero delta SoC points and line intersecting SoC at $60 \% \& \mathrm{SoC}$ at $90 \%$ 


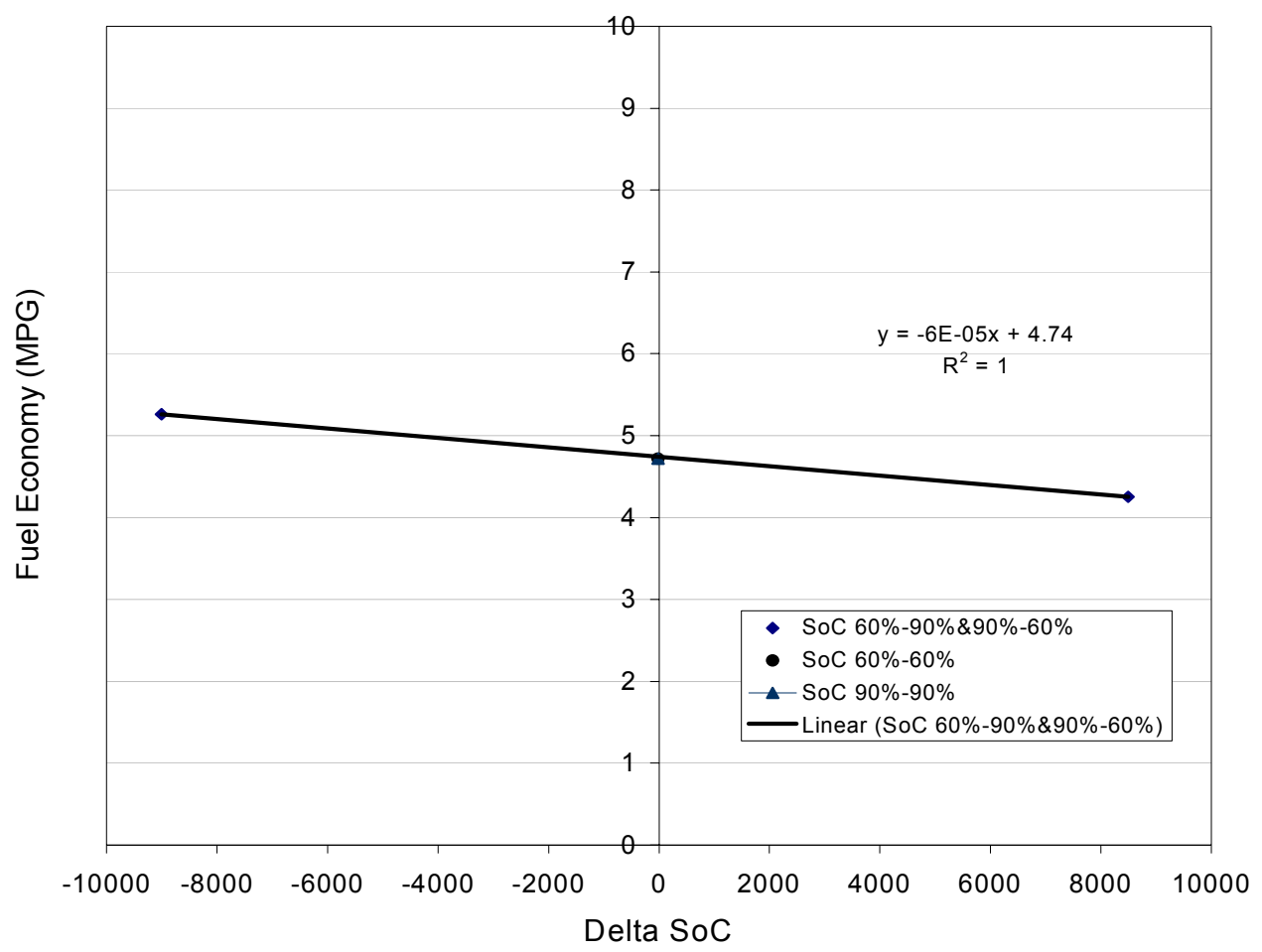

Figure 44: MPG of HEV with auxiliary load on UDDS cycle at $\mathrm{C1}=0.5$, with $26 \%$ difference between the points at zero delta $\mathrm{SoC}$ and line intersecting $\mathrm{SoC}$ at $60 \% \&$ SoC at $90 \%$ 


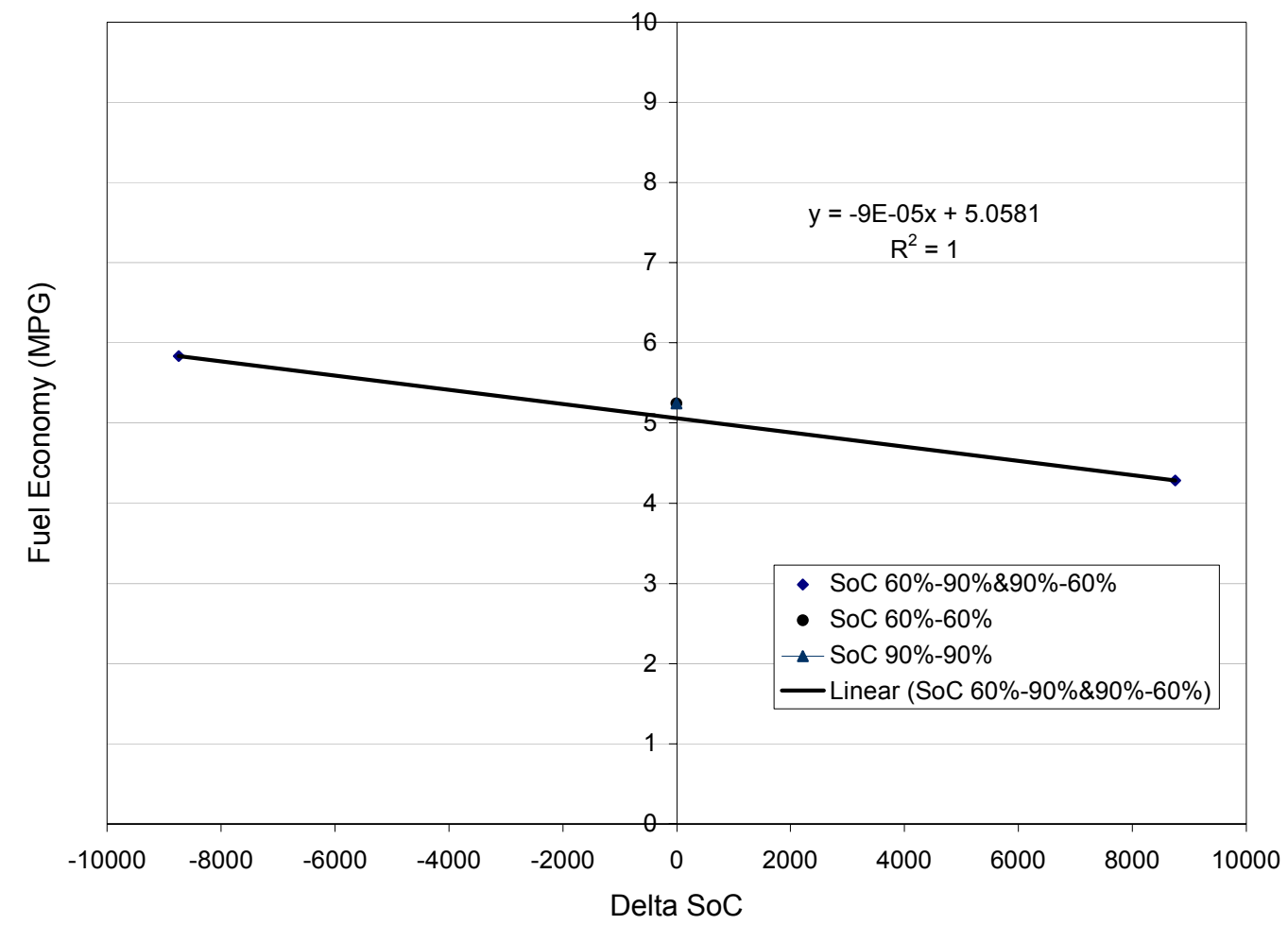

Figure 45: MPG of HEV without auxiliary load on UDDS1 cycle at $\mathrm{C} 1=0.3$, with $-3.6 \%$ difference between the zero delta points and line intersecting SoC at $60 \% \&$ SoC at $90 \%$ 


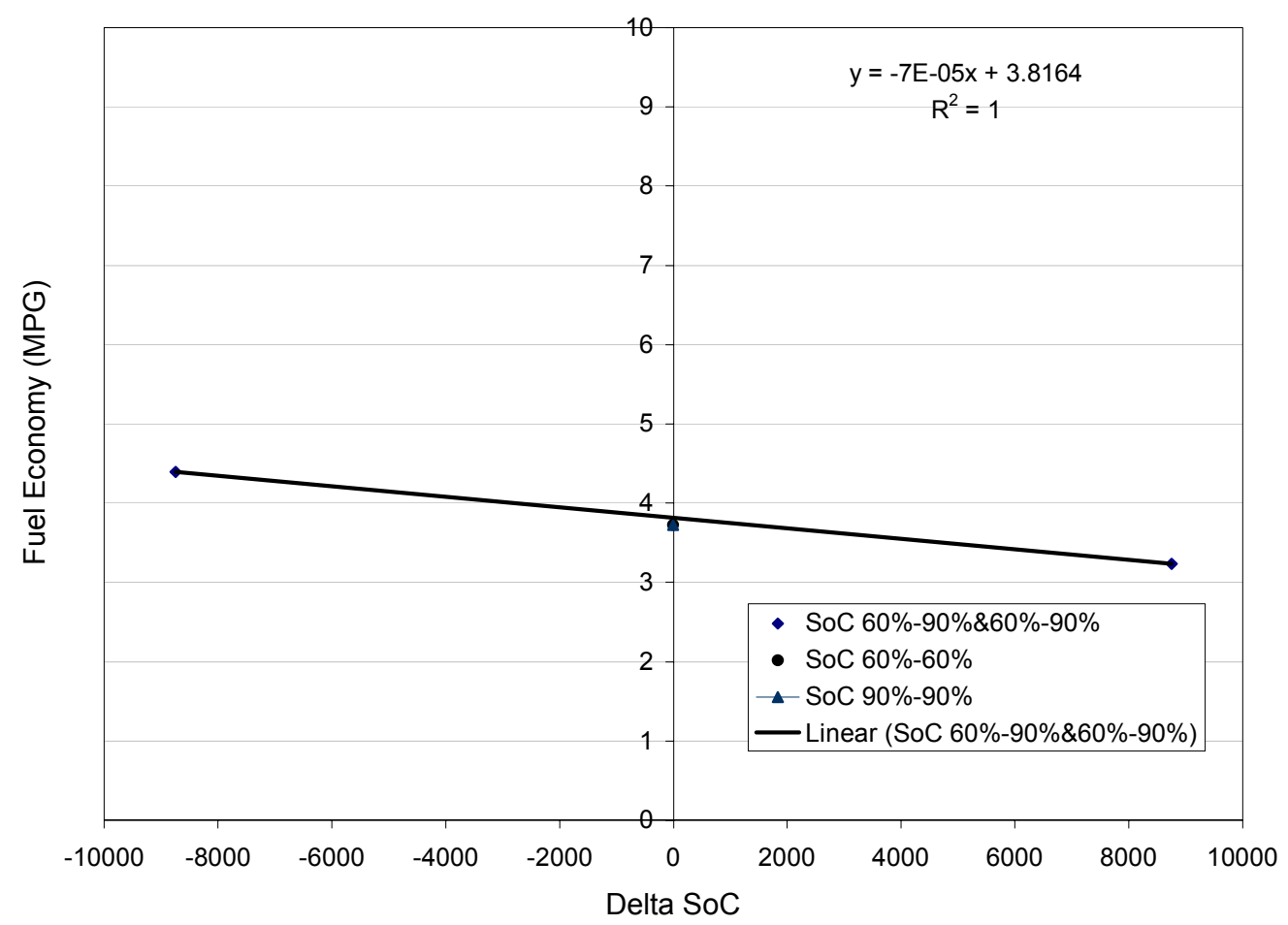

Figure 46: MPG of HEV with auxiliary load on UDDS1 cycle at $\mathrm{C1}=0.5$, with $2.4 \%$ difference between zero delta points and line intersecting $\mathrm{SoC}$ at $60 \%$ and $\mathrm{SoC}$ at $90 \%$ 


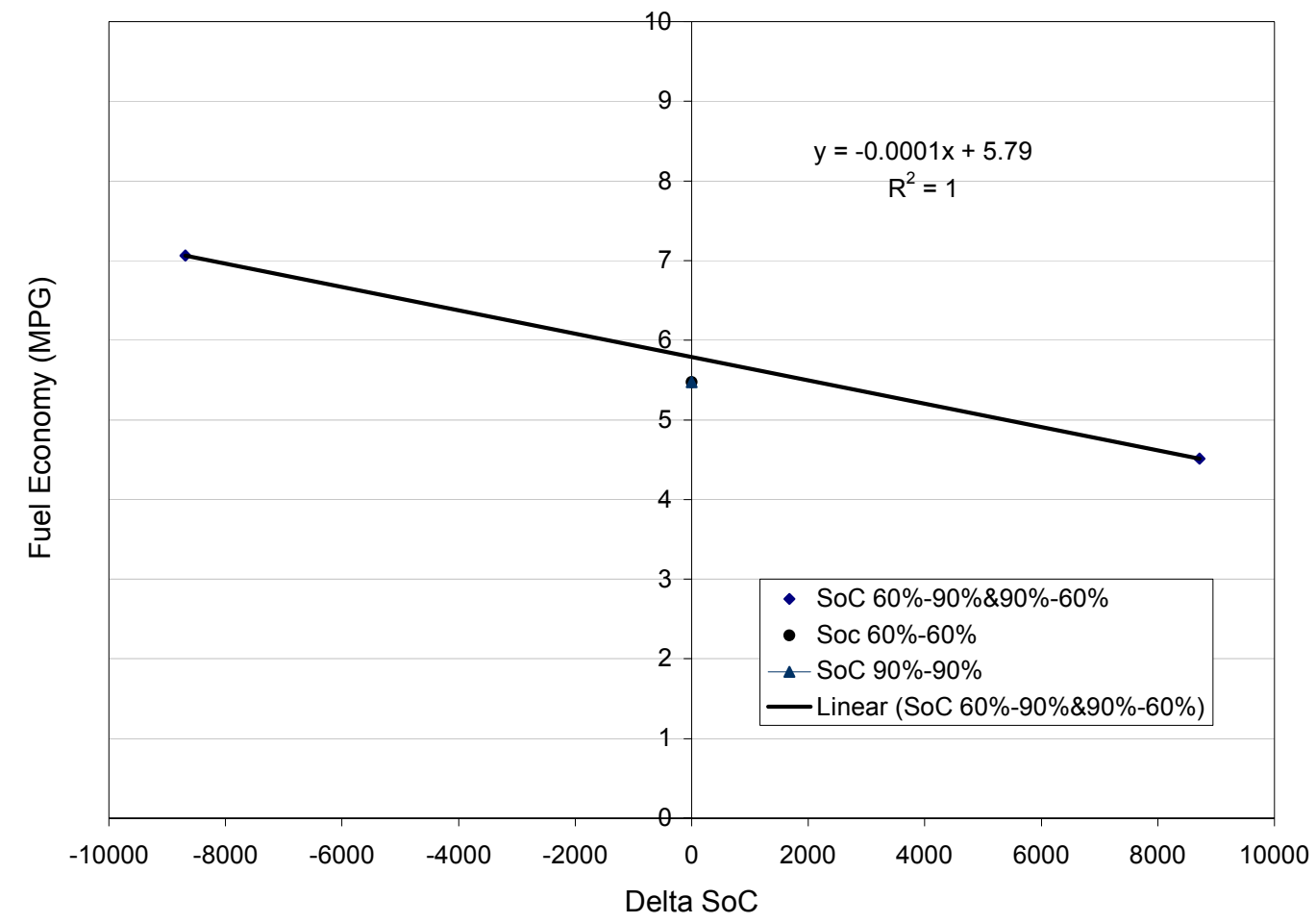

Figure 47: MPG of HEV on UDDS2 cycle without auxiliary load at $\mathrm{C} 1=0.5$, with $5.5 \%$ difference between zero delta SoC points and line intersecting SoC at $60 \%$ \& SoC at $90 \%$ 


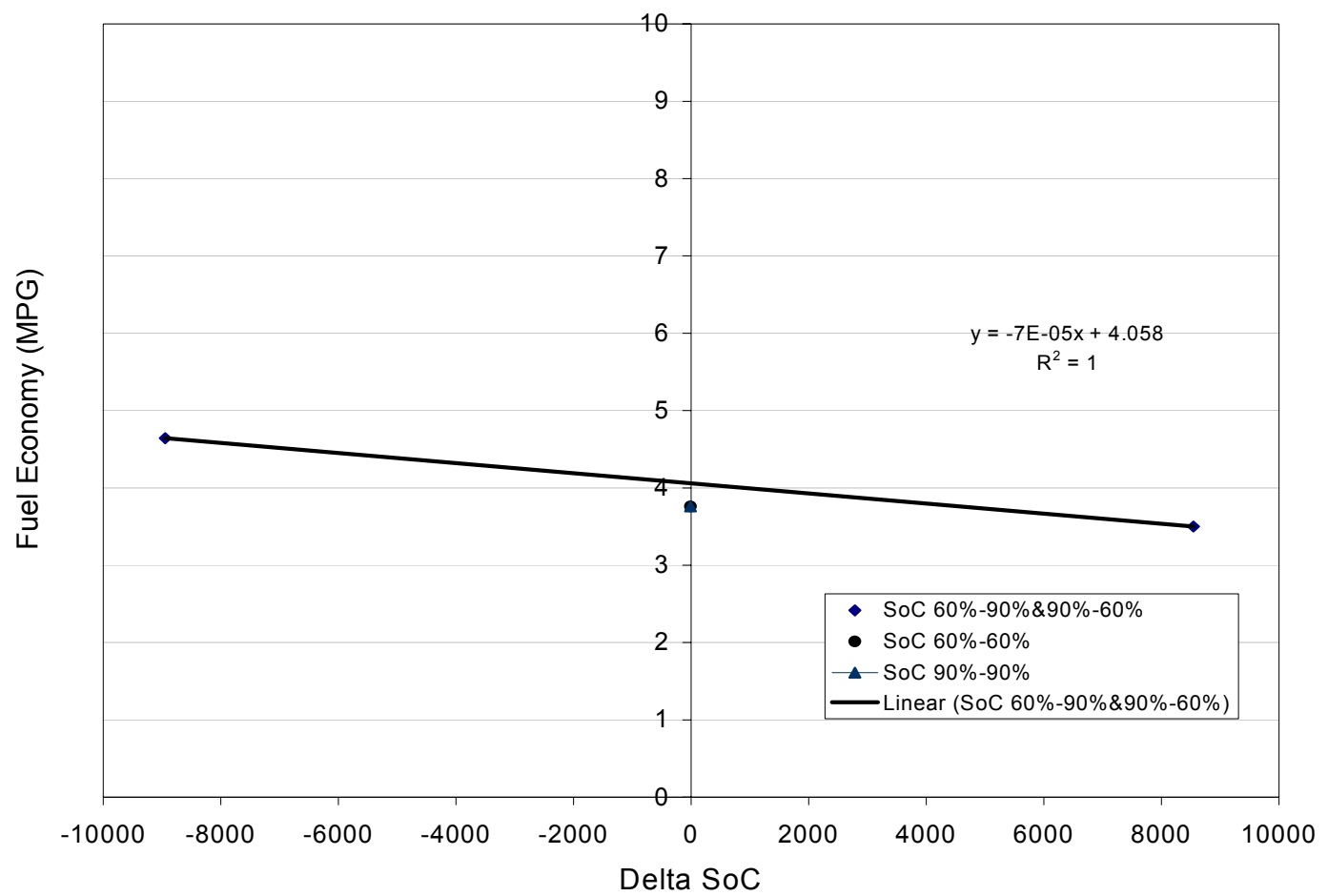

Figure 48: MPG of HEV on UDDS2 with auxiliary load for $\mathrm{C} 1=0.5$, with $7.3 \%$ difference between zero delta SoC points and line intersecting SoC at $60 \%$ \& SoC at $90 \%$

A vehicle that typically maintains its battery net SOC change within this differential is generally more load following and as a result battery losses are minimized. When a vehicle does not maintain this differential due to significant energy transfer through the batteries the efficiency of the batteries themselves has a pronounced effect on the vehicle efficiency and the relationship between net SOC and fuel economy (also emissions) becomes non-linear. 


\subsection{Parametric Study}

With change in vehicle parameters like mass and drag coefficient the fuel economy is studied. Tables 20 to 23 show the fuel economy data for different cycles for certain $\mathrm{C} 1$ and $\mathrm{C} 2$ values. Further simulations were run in ADVISOR for different mass and drag coefficients. Comparisons are done with the results of ADVISOR simulations and thesis simulations.

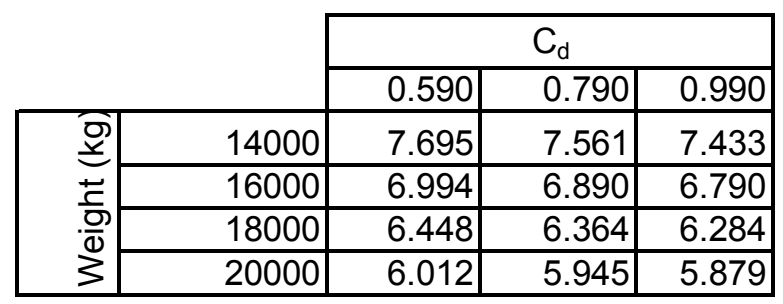

Table 20: Parametric study on CBD cycle, values in the table represents the fuel economy (MPG) data.

\begin{tabular}{|c|c|c|c|c|}
\hline & \multicolumn{3}{|c|}{$\mathrm{Cd}$} \\
\hline & & 0.590 & 0.790 & 0.990 \\
\hline \multirow{4}{*}{ 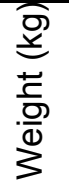 } & 14000 & 5.956 & 5.890 & 5.824 \\
\hline & 16000 & 5.314 & 5.262 & 5.210 \\
\hline & 18000 & 4.806 & 4.763 & 4.721 \\
\hline & 20000 & 4.394 & 4.358 & 4.324 \\
\hline
\end{tabular}

Table 21: Parametric study on Manhattan cycle, values in the table represents the fuel economy (MPG) data. 


\begin{tabular}{|c|c|c|c|c|}
\hline & \multicolumn{3}{|c|}{$\overline{C d}$} \\
\hline & & 0.590 & 0.790 & 0.990 \\
\hline \multirow{4}{*}{ 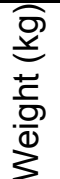 } & 14000 & 5.186 & 5.077 & 4.975 \\
\hline & 16000 & 5.007 & 4.913 & 4.823 \\
\hline & 18000 & 4.854 & 4.773 & 4.693 \\
\hline & 20000 & 4.727 & 4.656 & 4.589 \\
\hline
\end{tabular}

Table 22: Parametric study on CSHVC, values in the table represents the fuel economy (MPG) data.

\begin{tabular}{|c|c|c|c|c|}
\hline & \multicolumn{3}{|c|}{$\mathrm{Cd}$} \\
\hline & & 0.590 & 0.790 & 0.990 \\
\hline \multirow{4}{*}{ 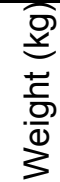 } & 14000 & 6.990 & 6.413 & 5.934 \\
\hline & 16000 & 6.500 & 6.015 & 5.610 \\
\hline & 18000 & 6.093 & 5.682 & 5.331 \\
\hline & 20000 & 5.755 & 5.398 & 5.092 \\
\hline
\end{tabular}

Table 23: Parametric study on UDDS cycle, values in the table represents the fuel economy (MPG) data.

For all the cycles with increase in vehicle mass and increase in coefficient in drag there is a decrease in fuel economy and vise versa. For the cycles with low speeds and more acceleration, the change in drag coefficient $\left(\mathrm{C}_{\mathrm{d}}\right)$ doesn't really affect the fuel economy. The change in fuel economy for CBD cycle is $1.5 \%$, for Manhattan cycle it is $0.9 \%$ and for CSHVC it is $1.8 \%$. But for the UDDS cycle where the speed of vehicle is high, fuel economy varied up to $8 \%$. When high accelerations are demanded, the inertial term dominates the power requirements. Change in fuel economy with change in vehicle mass for CBD cycle is around $8 \%$, for Manhattan cycle it is around $10 \%$, for UDDS cycle it is around 6\% and for CSHVC it is around 3\%. At low speeds, rolling resistance is quite significant compared to aerodynamic drag, but at high speeds, the $\mathrm{V}^{3}$ term causes aerodynamic drag to dominate. Figures 49 to 52 show the simulations run in advisor. 

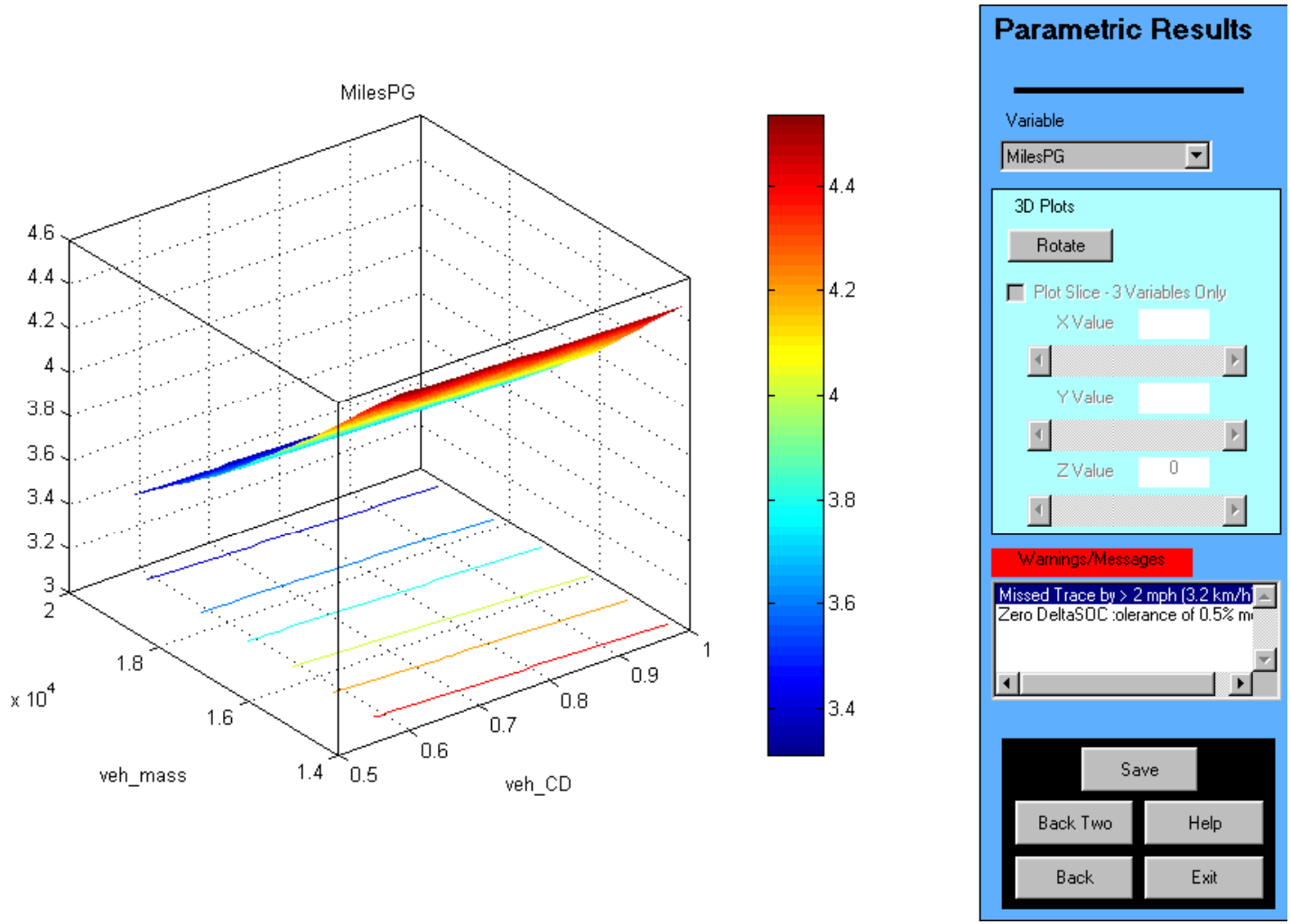

Figure 49: Advisor simulation on UDDS cycle 

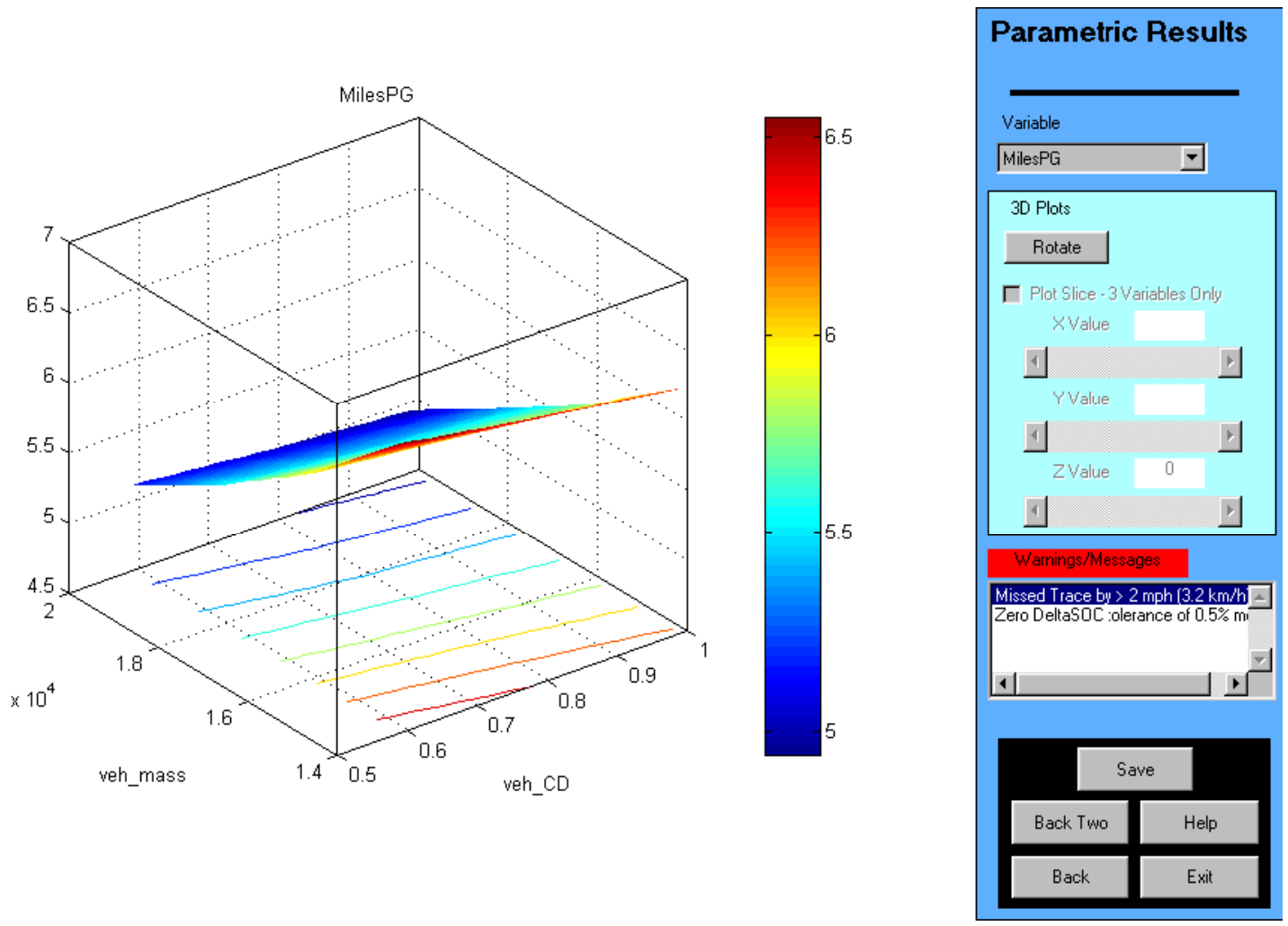

Figure 50: Advisor simulation on CSHVC 

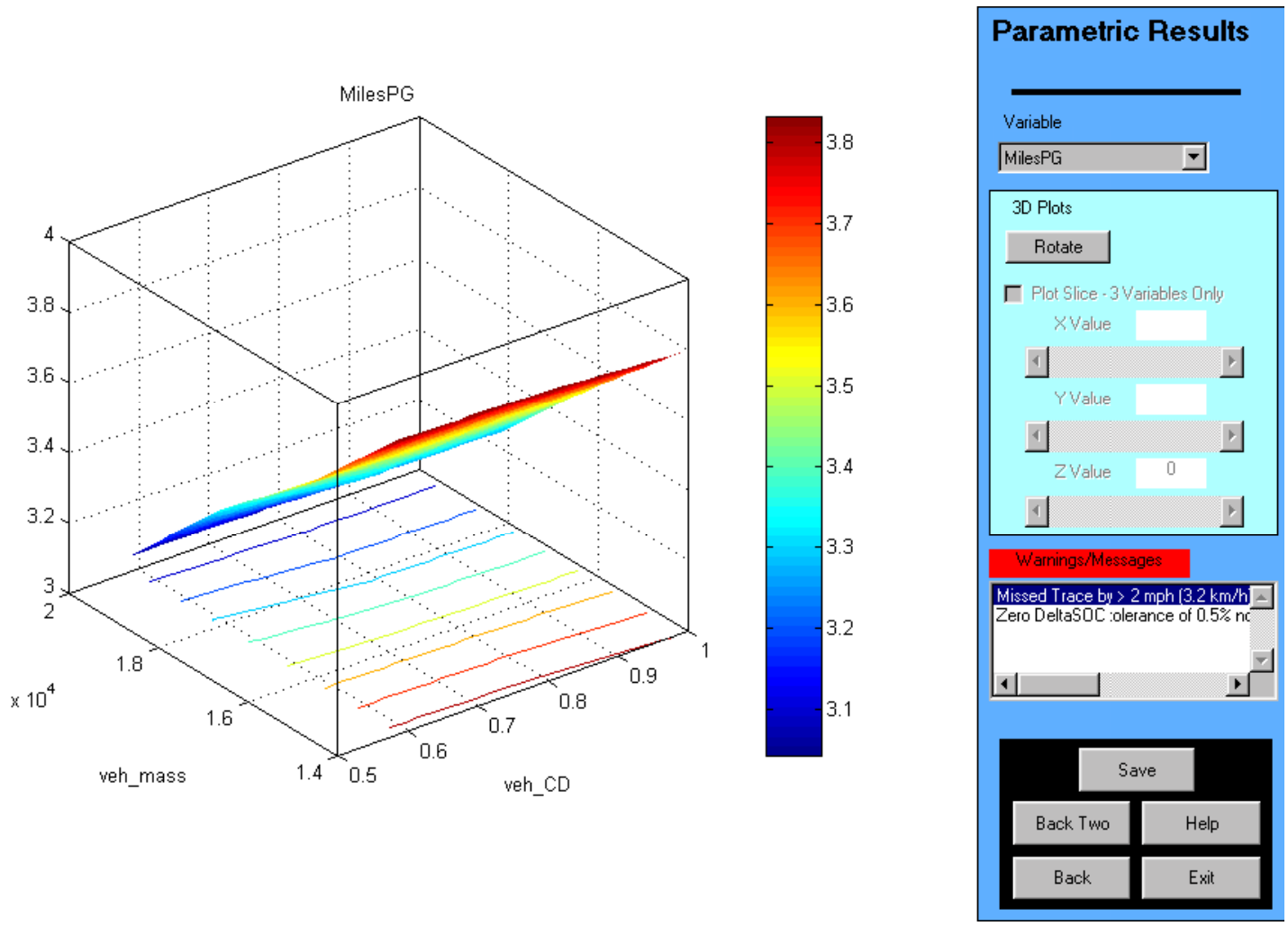

Figure 51: Advisor simulation on Manhattan cycle 

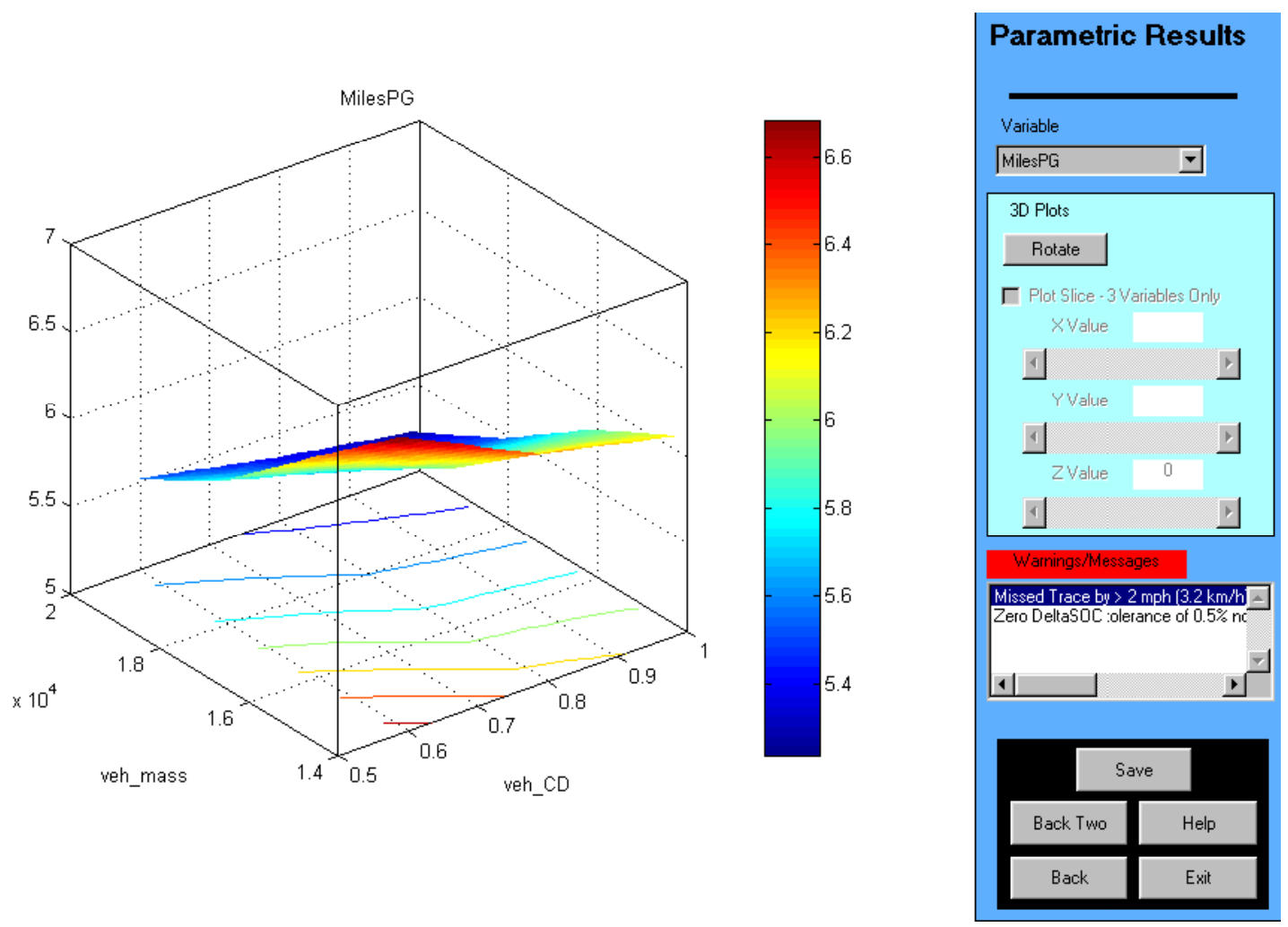

Figure 52: Advisor simulation on CBD cycle

The simulations run in ADVISOR for parametric study show that there is increase in the fuel economy with decrease in vehicle mass and drag coefficient. These results from ADVISOR model vary with those from simulation model by $2 \%$ for CSHV cycle, by $4 \%$ for UDDS cycle, by $15 \%$ for CBD cycle and by $48 \%$ for Manhattan cycle. This is mainly due to the difference in control strategy used. In ADVISOR the ICE is turned off when needed but in the thesis simulation model the ICE is always on. 


\section{Emissions Model Development}

The major exhaust gases from a diesel vehicle are $\mathrm{CO}_{2}, \mathrm{CO}, \mathrm{HC}, \mathrm{PM}$ and $\mathrm{NO}_{\mathrm{x}}$. Carbon Dioxide $\left(\mathrm{CO}_{2}\right)$ is the product of complete combustion of the fuel. This gas is produced when anything organic is burned, and has no direct health effects; in fact the human body produces it when you breathe out. However $\mathrm{CO}_{2}$ plays an important role in adding to the greenhouse effect, which keeps the earth warm. In turn this is adding to global warming. Carbon monoxide (CO) results from the incomplete burning of fuel, which can have direct health effects. When inhaled, $\mathrm{CO}$ reduces the oxygen carrying capacity of the blood and can cause headaches, fatigue, stress, respiratory problems and very high levels of mortality. Hydrocarbons are unburnt or partially burnt fuel particles in vaporized form. Hydrocarbons are compounds made of hydrogen and carbon; these include petrol, diesel, gas and some solvents. HCs react with oxides of nitrogen in sunlight to produce a number of harmful compounds called photochemical oxidants. These include peroxacetyl nitrate (PAN) and ozone, both of which are irritating to humans and cause plant damage. Particulates is a general term used to describe tiny bits of matter (technically between 0.1 and 25 thousandths of a millimeter) floating around in the atmosphere, such as certain types of smoke (like diesel smoke), fine ash and dust. In urban areas, 5 to 20 percent of particulates are various sulphates, which are believed to be responsible for increased asthma attacks, aggravation of any existing heart and lung disease, and a lowered resistance to breathing problems in children. Oxides of nitrogen (NOx), these are produced whenever fuel is burned. Reacting with HCs NOx emissions are further oxidised in the atmosphere contributing to the production of acid rain [11]. Inorder to meet the EPA standards of reduced emissions various technologies are 
developed. Some solutions to the exhaust gases reduction are increase in the engine efficiency, use of fuel with less sulphur content and use of electric and hybrid electric vehicles.

The EPA tests engines manufactured for emissions. Emissions of a vehicle can be measured by performing chassis dynamometer testing over various driving cycles as given in section 1.3 of this thesis. The continuous emissions from the chassis tests preformed on any vehicle would have time delay with respect to axle power. The exhaust gases are measured at the end of dilution tunnel connected to the exhaust of the vehicle. Where as the axle power is measured instantaneously. Here in this thesis emission modeling was considered for NOx. Continuous emissions data were shifted to compensate for the time delay before data evaluations were performed. A crosscorrelation numeric evaluation was performed on all continuous gaseous, Tapered Element Oscillating Micro balance (TEOM) for PM, and tunnel flow rate data with respect to continuous engine power data to determine the time shift necessary to compensate for the time delay and to assure proper data correlation [19]. Since a backwards dispersion model would require assumptions and inaccuracies, the dispersion model was applied to engine data (i.e. speed and torque) as if these parameters "traveled" with the exhaust gases to the analyzers. Ramamurthy [20,21] showed this approach to yield an improved correlation between NOx and axle power from transient chassis tests of heavy-duty vehicles. But for a HEV, the axle power is due to the engine power and battery power. So dispersion of axle power would not give appropriate correlation between NOx and engine power. As the engine power data is not available from WVU THDVETL an attempt to model NOx emissions was made by studying the correlation 
between $\mathrm{CO}_{2}$ and NOx. Fuel consumed is directly proportional to engine power. Fuel consumed is also directly proportional to $\mathrm{CO}_{2}$ by the combustion equation show in Equation $22[22]$.

$$
\mathrm{C}_{n} \mathrm{H}_{1.8 n}+\left(n+\frac{1.8 n}{4}\right) \mathrm{O}_{2} \rightarrow n \mathrm{CO}_{2}+\frac{1.8 n}{2} \mathrm{H}_{2} \mathrm{O}
$$

where $n$ is the number of moles and $C_{n} H_{1.8 n}$ is light diesel.

Same vehicle run on two different cycles was considered for modeling. Each cycle has three different runs. The vehicle considered was a transit bus 6353 run on two different cycles (CBD cycle and Manhattan cycle).

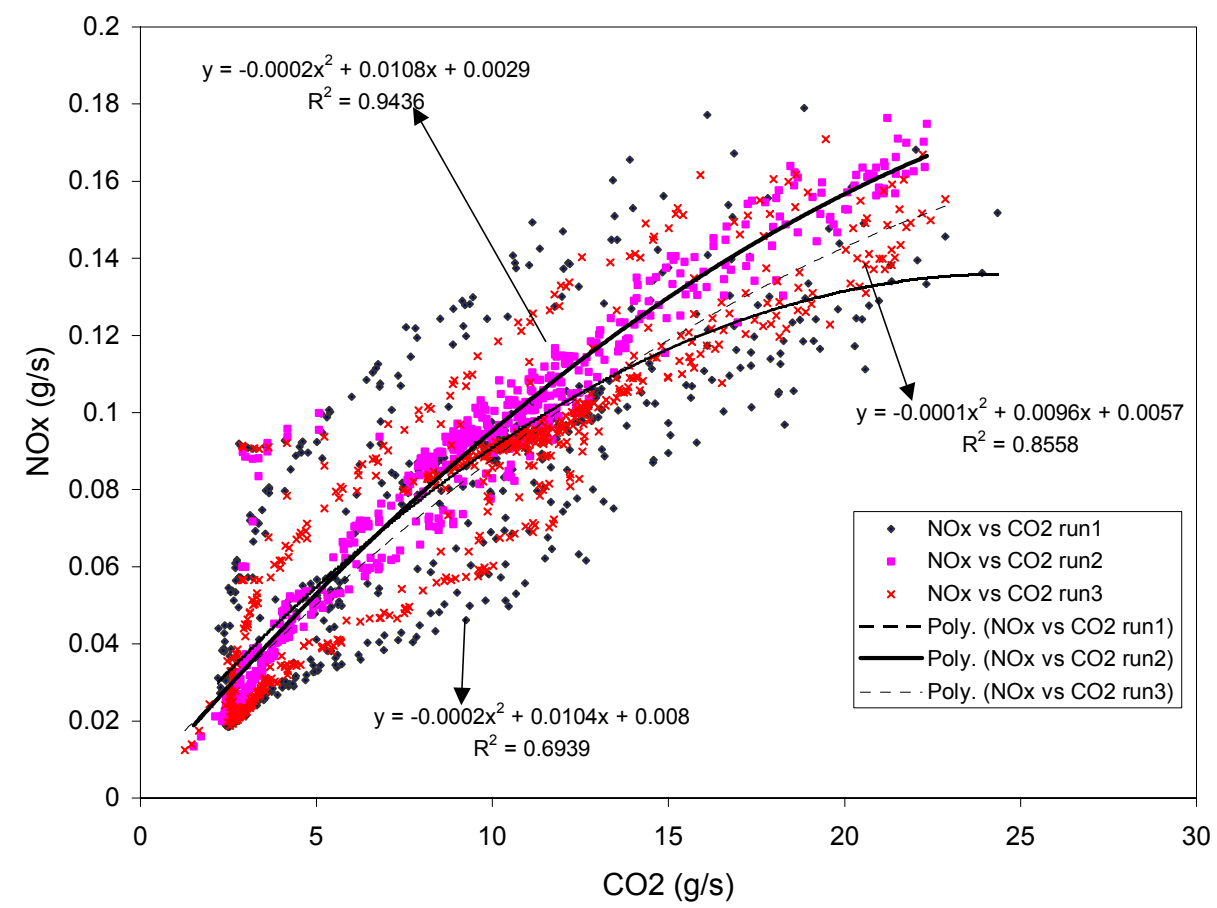

Figure 53: $\mathrm{NOx}$ vs $\mathrm{CO}_{2}$ for three consecutive runs on CBD cycle for vehicle 6353.

In the Figure $53 \mathrm{NOx}$ is shown as function of $\mathrm{CO}_{2}$ and is given by

$$
\mathrm{NO}_{\mathrm{x}}=\mathrm{a}\left(\mathrm{CO}_{2}\right)^{2}+\mathrm{b}\left(\mathrm{CO}_{2}\right)+\mathrm{c}
$$


The coefficients $\mathrm{a}, \mathrm{b}$ and $\mathrm{c}$ are tabulated in the Table 24 for three different runs on a CBD cycle. Based on the values of the coefficients for the equation NOx is predicted as a function of $\mathrm{CO}_{2}$.

\begin{tabular}{|l|r|r|r|}
\cline { 2 - 4 } \multicolumn{1}{c|}{} & a & b & c \\
\hline Run 1 & -0.0002 & 0.0108 & 0.0029 \\
\hline Run 2 & -0.0002 & 0.0104 & 0.008 \\
\hline Run 3 & -0.0001 & 0.0096 & 0.0057 \\
\hline Average & $\mathbf{- 0 . 0 0 0 2}$ & $\mathbf{0 . 0 1 0 3}$ & $\mathbf{0 . 0 0 5 5}$ \\
\hline Std & $\mathbf{0 . 0 0 0 1}$ & $\mathbf{0 . 0 0 0 6}$ & $\mathbf{0 . 0 0 2 6}$ \\
\hline \%CV & $\mathbf{- 3 4 . 6 4}$ & $\mathbf{5 . 9 5}$ & $\mathbf{4 6 . 1 6}$ \\
\hline
\end{tabular}

Table 24: Values of coefficients for the relation $\mathrm{NOx}$ as a function of $\mathrm{CO}_{2}$

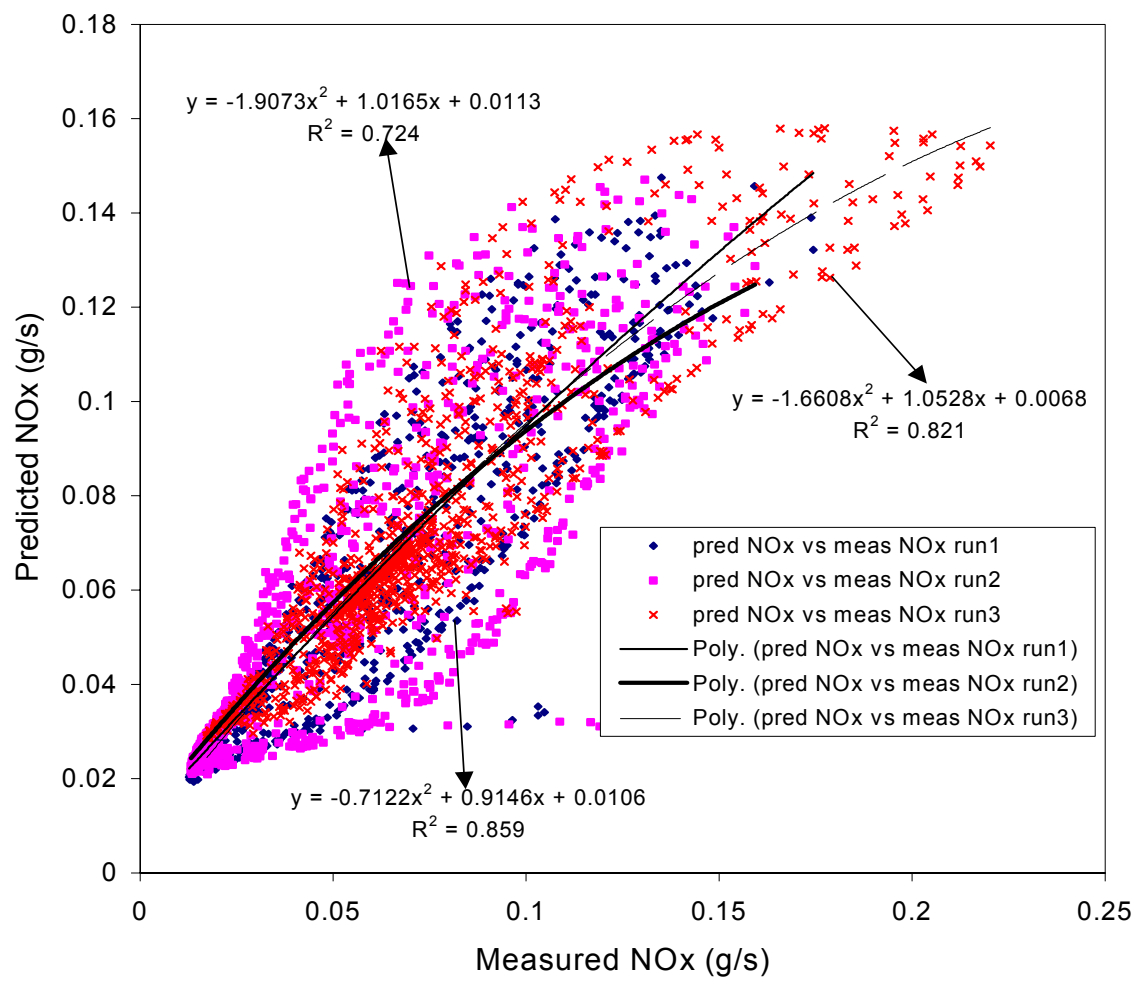

Figure 54: NOx vs $\mathrm{CO}_{2}$ for three different runs on Manhattan cycle for vehicle 6353. 
Based on the equation for $\mathrm{NOx}$ as function of $\mathrm{CO}_{2}$ for a transit bus over $\mathrm{CBD}$ cycle, NOx has been predicted for the Manhattan cycle. $\mathrm{R}^{2}$ values in Figure 54 show that there is a strong correlation between the predicted NOx and the measured NOx. So NOx can be predicted as function of $\mathrm{CO}_{2}$ and $\mathrm{NOx}$ can be predicted with this relation over different driving cycles for the same vehicle. 


\section{Conclusions}

In this thesis a control strategy has been developed for the power flow from the engine based on the Battery State of charge. In order to evaluate the performance of the hybrid electric vehicles, the fuel economy data were corrected for state of charge to account for fluctuations in the battery SoC. The fuel economy data thus obtained from various driving cycles has been compared with those of the in-use HEVs (Orion VI buses). ADVISOR simulation models and the thesis simulation models both yielded results close to the in-use HEVs.

The fuel economy of HEV depends on the order of the events in the cycle and this was shown in cycles UDDS, UDDS1 and UDDS2. The parameters of the vehicle contribute an extent for fuel economy. Low mass, low rolling resistance and low drag coefficient give good fuel economy. With the use of auxiliary load, fuel consumption was increases to a considerable extent. For different values of $\mathrm{C} 1$ in the control strategy, the effects of auxiliary load have been studied.

As NOx emissions are of primary concern with ICE, a modeling effort has been made to predict NOx. For a vehicle (transit bus) NOx was predicted based on $\mathrm{CO}_{2}$ over CBD cycle. Further NOx emissions were predicted for the same vehicle over Manhattan cycle based on the relation obtained from CBD cycle. It was observed that there was a strong correlation with $\mathrm{R}^{2}$ value of 0.724 between the predicted $\mathrm{NOx}$ and the measured NOx, which was shown over Manhattan cycle. 


\section{References}

[1] http://www.ta.doc.gov/pngv, The Partnership for a New Generation of Vehicles, May 2001.

[2] http://www.osti.gov/hvt/21stcenturytruck.pdf, Technology Roadmap for the $21^{\text {st }}$ Century Truck Program, May 2001.

[3] An, Fen., Vyas, Anant., Anderson, John., and Santini, Danilo.; "Evaluating Commercial and Prototype HEVs", SAE Paper 2001-01-0951.

[4] "Hybrid-Electric Drive Heavy-Duty Vehicle Testing Project - Final Emissions Report", NAVC, DARPA, Northeast Advanced Vehicle Consortium, M. J. Bradley \& Associates, Inc., and West Virginia University, 2000.

[5] http://www.geocities.com/Motorcity/Factory/1723/History_of_Evs.htm, History of EV’s May 2001.

[6] Tóth-Nagy, Csaba.; "Investigation and Simulation of the Planetary Combination Hybrid Electric Vehicle", Masters Thesis, West Virginia University, 2000.

[7] Parsley, William.; "New York City Transit Operating Experience with Hybrid Transit Buses", SAE / NESEA Hybrid Electric Vehicles in the Bus and Truck Markets TOPTEC: New Ways of Building Better Heavy Duty Vehicles, May 11-12, 2000.

[8] http://www.ctts.nrel.gov/analysis, Vehicle Systems Analysis, ADVISOR 3.2, June 2001.

[9] Clark, N. N., Conley, Jason., Jarrett, Ronald P., Nennelli, Anjali., and Tóth-Nagy, Csaba.; "Emissions Modeling of Heavy-Duty Conventional and Hybrid Electric Vehicles", SAE Paper 2001-01-3675. 
[10] Ganesan, Baskaran., and Clark, N. N.; "Relationships Between Instantaneous And Measured Emissions in Heavy-Duty Applications”, SAE Paper 2001-01-3536.

[11] http://www.soton.ac.uk/ engenvir/environment/transport/exhaust.htm, Exhaust Gases, June 2001.

[12] http://www.dieselnet.com, Heavy-Duty Truck and Bus Engines, June 2001.

[13] http://www.trucks.doe.gov/plain-talk/regs.html, EPA, June 2001.

[14] Clark, N. N., McKain, D. L., Balon, T. H., Moynihan, P. J., Lynch, S. A., and Webb, T. C.; "Characterization of Emissions from Hybrid-Electric and Conventional Buses", SAE Paper 2000-01-2011.

[15] Clark, N. N., Daley, J. J., Nine, R. D., and Atkinson, C. M.; “Application of the New City-Suburban Heavy Vehicle Route (CSHVR) to Truck Emissions Characterization”, SAE Paper 1999-01-1467.

[16] Taylor, Wayne T.; "Feasible Design Strategies for Near-Term Hybrid Electric Vehicles”, Masters Thesis, West Virginia University, 1997.

[17] Kellermeyer, W. F.; "Development and Validation of a Modular Hybrid Electric Vehicle Simulation Model”, Masters Thesis, West Virginia University, 1998.

[18] http://www.hepi.com/xe.htm, For HEV Applications, July 2001.

[19] Messer, J. T., Clark, N. N., and Lyons, D. W.; "Measurement Delays and Modal Analysis For a Heavy Duty Transportable Laboratory”, SAE 1995-95-0218.

[20] Ramamurthy, R., Clark, N. N., Atkinson, C. M., and Lyons, D.W.; "Models for Predicting Transient Heavy Duty Vehicle Emissions”, SAE 1998-98-2652.

[21] Ramamurthy, R., and Clark, N. N.; "Atmospheric Emissions Inventory Data for Heavy-Duty Vehicles", Environ. Sci. Technol. 1999, 33, 55-62. 
[22] Heywood, John B.; Internal Combustion Engine Fundamentals, McGraw-Hill; 1998, p 915. 


\section{Appendix}

Tables 25 through 36 show the SoC corrections and the errors over various driving cycles. In the tables $27,31,33,35$ and 36 the fuel economy values for SoC for $\mathrm{C} 1=0.8$ and $\mathrm{C} 1=0.9$ are not tabulated, for higher values of $\mathrm{C} 1$ the values of $\mathrm{C} 2$ tend to very small and also it becomes very tough to maintain the battery in charge sustaining condition practically. 


\begin{tabular}{|c|c|c|c|c|c|}
\hline & & \multicolumn{2}{|c|}{ w/o auxiliary load } & & \\
\hline C1 & $\mathrm{C} 2$ & SoC & delta SoC & MPG & \\
\hline 0.1 & $4.799 * 10-2$ & $60-60$ & -0.010 & 6.849 & \\
\hline 0.1 & $18.398 * 10$ & $90-90$ & 0.000 & 4.817 & \\
\hline 0.1 & $3.5 * 10-2$ & $60-90$ & 8555 & 5.728 & \\
\hline 0.1 & $18.389 * 10$ & $90-60$ & -8748 & 7.178 & \\
\hline \multicolumn{5}{|c|}{ Average of Fuel economy at $60 \%-60 \% \& 90 \%-90 \%$ SoC } & 5.833 \\
\hline \multicolumn{5}{|c|}{ Y intercept of $60 \%-90 \%$ and $90 \%-60 \%$ SoC } & 6.445 \\
\hline \multicolumn{5}{|l|}{$\%$ Error } & 9.493 \\
\hline \multirow{5}{*}{$\frac{0 .}{0 .}$} & $\mathrm{C} 2$ & SoC & delta SoC & MPG & \\
\hline & $4.1^{*} 10-2$ & $60-60$ & 0.520 & 6.294 & \\
\hline & $17.41 * 10-2$ & $90-90$ & -0.030 & 7.166 & \\
\hline & $4.1 * 10-2$ & $60-90$ & 8749 & 6.849 & \\
\hline & $17.41 * 10-2$ & $90-60$ & -8748 & 4.805 & \\
\hline \multicolumn{5}{|c|}{ Average of Fuel economy at $60 \%-60 \% \& 90 \%-90 \%$ SoC } & 6.730 \\
\hline \multicolumn{5}{|c|}{$\mathrm{Y}$ intercept of $60 \%-90 \%$ and $90 \%-60 \%$ SoC } & 5.827 \\
\hline \multicolumn{5}{|l|}{$\%$ Error } & -15.50 \\
\hline $\mathrm{C} 1$ & $\mathrm{C} 2$ & SoC & delta SoC & MPG & \\
\hline 0.3 & $3.323 * 10-2$ & $60-60$ & -0.010 & 6.862 & \\
\hline 0.3 & $16.41 * 10-2$ & $90-90$ & -0.020 & 4.801 & \\
\hline 0.3 & $3.3^{*} 10-2$ & $60-90$ & 8745 & 5.694 & \\
\hline 0.3 & $17.41 * 10-2$ & $90-60$ & -8736 & 7.163 & \\
\hline \multicolumn{5}{|c|}{ Average of Fuel economy at $60 \%-60 \% \& 90 \%-90 \%$ SoC } & 5.831 \\
\hline \multicolumn{5}{|c|}{ Y intercept of $60 \%-90 \%$ and $90 \%-60 \%$ SoC } & 6.429 \\
\hline \multicolumn{5}{|l|}{$\%$ Error } & 9.295 \\
\hline C1 & $\mathrm{C} 2$ & SoC & delta SoC & MPG & \\
\hline 0.4 & $2.463 * 10-2$ & $60-60$ & 0.060 & 6.890 & \\
\hline 0.4 & $15.372 * 10$ & $90-90$ & 0.050 & 4.802 & \\
\hline 0.4 & $2.463 * 10-2$ & $60-90$ & 8748 & 5.248 & \\
\hline 0.4 & $15.372 * 10$ & $90-60$ & -8748 & 7.163 & \\
\hline \multicolumn{5}{|c|}{ Average of Fuel economy at $60 \%-60 \% \& 90 \%-90 \%$ SoC } & 5.846 \\
\hline \multicolumn{5}{|c|}{ Y intercept of $60 \%-90 \%$ and $90 \%-60 \%$ SoC } & 6.205 \\
\hline \multicolumn{5}{|l|}{$\%$ Error } & 5.791 \\
\hline $\mathrm{C} 1$ & $\mathrm{C} 2$ & SoC & delta SoC & MPG & \\
\hline 0.5 & $1.488 * 10-2$ & $60-60$ & 0.030 & 6.935 & \\
\hline 0.5 & $14.27^{*} 10-2$ & $90-90$ & 0.000 & 4.811 & \\
\hline 0.5 & $1.56 * 10-2$ & $60-90$ & 8749 & 4.989 & \\
\hline 0.5 & $15.372 * 10$ & $90-60$ & -8733 & 7.170 & \\
\hline \multicolumn{5}{|c|}{ Average of Fuel economy at $60 \%-60 \% \& 90 \%-90 \%$ SoC } & 5.873 \\
\hline \multicolumn{5}{|c|}{$Y$ intercept of $60 \%-90 \%$ and $90 \%-60 \%$ SoC } & 6.081 \\
\hline \multicolumn{5}{|c|}{$\%$ Error } & 3.411 \\
\hline
\end{tabular}

Table 25: SoC correction for HEV w/o auxiliary load over CBD cycle (continued in next page) 


\begin{tabular}{|c|c|c|c|c|c|}
\hline C1 & $\mathrm{C} 2$ & SoC & delta SoC & MPG & \\
\hline 0.6 & $0.339 * 10-2$ & $60-60$ & -0.270 & 7.000 & \\
\hline 0.6 & $13.09 * 10-2$ & $90-90$ & -0.110 & 4.829 & \\
\hline 0.6 & $0.93^{*} 10-2$ & $60-90$ & 8748 & 4.878 & \\
\hline 0.6 & $13.09 * 10-2$ & $90-60$ & -8748 & 7.186 & \\
\hline Average of & Fuel econo & omy at & $-60 \% \& 90 \%$ & $0-90 \%$ SoC & 5.915 \\
\hline $\mathrm{Y}$ intercept & of $60 \%-90^{\circ}$ & $\%$ and $s$ & $-60 \%$ SoC & & 6.032 \\
\hline$\%$ Error & & & & & 1.948 \\
\hline C1 & $\mathrm{C} 2$ & SoC & delta SoC & MPG & \\
\hline 0.7 & $11.3^{*} 10-2$ & $60-60$ & 464.0 & 6.805 & \\
\hline 0.7 & $11.8 * 10-2$ & $90-90$ & -0.020 & 4.858 & \\
\hline 0.7 & $0.61 * 10-2$ & $60-90$ & 8753 & 4.874 & \\
\hline 0.7 & $13.09 * 10-2$ & $90-60$ & -8729 & 7.205 & \\
\hline Average of & Fuel econo & omy at & $-60 \% \& 90 \%$ & $0-90 \%$ SoC & 5.832 \\
\hline Y intercept & of $60 \%-90^{\circ}$ & $\%$ and $s$ & $-60 \%$ SoC & & 6.041 \\
\hline$\%$ Error & & & & & 3.470 \\
\hline C1 & $\mathrm{C} 2$ & SoC & delta SoC & MPG & \\
\hline 0.8 & $10 * 10-2$ & $60-60$ & 487.4 & 6.852 & \\
\hline 0.8 & $10.32 * 10-2$ & $90-90$ & -0.070 & 4.898 & \\
\hline 0.8 & $0.41 * 10-2$ & $60-90$ & 8775 & 4.905 & \\
\hline 0.8 & $10.32 * 10-2$ & $90-60$ & -8748 & 7.243 & \\
\hline Average of & Fuel econo & omy at & $-60 \% \& 90 \%$ & $0-90 \%$ SoC & 5.875 \\
\hline Y intercept & of $60 \%-90^{\circ}$ & $\%$ and $s$ & $-60 \%$ SoC & & 6.076 \\
\hline$\%$ Error & & & & & 3.305 \\
\hline C1 & $\mathrm{C} 2$ & SoC & delta SoC & MPG & \\
\hline 0.9 & $17.6 * 10-2$ & $60-60$ & 503.9 & 6.891 & \\
\hline 0.9 & $8.54 * 10-2$ & $90-90$ & 0.070 & 4.814 & \\
\hline 0.9 & $0.257^{*} 10-2$ & $60-90$ & 8781 & 4.964 & \\
\hline 0.9 & $10.25 * 10-2$ & $90-60$ & -8722 & 7.277 & \\
\hline Average of & Fuel econo & omy at & $-60 \% \& 90 \%$ & $0-90 \%$ SoC & 5.852 \\
\hline$Y$ intercept & of $60 \%-90^{\circ}$ & $\%$ and 5 & $-60 \%$ SoC & & 6.124 \\
\hline$\%$ Error & & & & & 4.440 \\
\hline
\end{tabular}

Table 25: SoC correction for HEV w/o auxiliary load over CBD cycle (continued) 


\begin{tabular}{|c|c|c|c|c|c|}
\hline & & \multicolumn{2}{|c|}{ with auxiliary load } & & \\
\hline C1 & $\mathrm{C} 2$ & SoC & delta SoC & MPG & \\
\hline 0.1 & $17.7^{*} 10-2$ & $60-60$ & -0.010 & 4.811 & \\
\hline 0.1 & $17.7 * 10-2$ & $90-90$ & -0.010 & 4.811 & \\
\hline 0.1 & $3.4 * 10-2$ & $60-90$ & 8159 & 4.554 & \\
\hline 0.1 & $18.389 * 10$ & $90-60$ & -8736 & 7.173 & \\
\hline \multicolumn{5}{|c|}{ Average of Fuel economy at $60 \%-60 \% \& 90 \%-90 \%$ SoC } & 4.811 \\
\hline \multicolumn{5}{|c|}{ Y intercept of $60 \%-90 \%$ and $90 \%-60 \%$ SoC } & 5.818 \\
\hline \multicolumn{5}{|l|}{$\%$ Error } & 17.31 \\
\hline C1 & $\mathrm{C} 2$ & SoC & delta SoC & MPG & \\
\hline 0.2 & $16.37^{* 10-2}$ & $60-60$ & 0.000 & 4.797 & \\
\hline 0.2 & $16.37^{*} 10-2$ & $90-90$ & 0.000 & 4.797 & \\
\hline 0.2 & $3 * 10-2$ & $60-90$ & 8151 & 4.421 & \\
\hline 0.2 & $16.37 * 10-2$ & $90-60$ & -8748 & 7.161 & \\
\hline \multicolumn{5}{|c|}{ Average of Fuel economy at $60 \%-60 \% \& 90 \%-90 \%$ SoC } & 4.797 \\
\hline \multicolumn{5}{|c|}{ Y intercept of $60 \%-90 \%$ and $90 \%-60 \%$ SoC } & 5.742 \\
\hline \multicolumn{5}{|l|}{$\%$ Error } & 16.46 \\
\hline C1 & $\mathrm{C} 2$ & SoC & delta SoC & MPG & \\
\hline 0.3 & $14.81 * 10-2$ & $60-60$ & 0.010 & 4.787 & \\
\hline 0.3 & $14.81 * 10-2$ & $90-90$ & 0.010 & 4.787 & \\
\hline 0.3 & $2.3^{*} 10-2$ & $60-90$ & 8035 & 4.220 & \\
\hline 0.3 & $16.37^{* 10-2}$ & $90-60$ & -8725 & 7.151 & \\
\hline \multicolumn{5}{|c|}{ Average of Fuel economy at $60 \%-60 \% \& 90 \%-90 \%$ SoC } & 4.787 \\
\hline \multicolumn{5}{|c|}{ Y intercept of $60 \%-90 \%$ and $90 \%-60 \%$ SoC } & 5.625 \\
\hline \multicolumn{5}{|l|}{$\%$ Error } & 14.90 \\
\hline C1 & $\mathrm{C} 2$ & SoC & delta SoC & MPG & \\
\hline 0.4 & $12.966 * 10$ & $60-60$ & 0.240 & 4.784 & \\
\hline 0.4 & $12.966 * 10$ & $90-90$ & 0.240 & 4.784 & \\
\hline 0.4 & $2 * 10-2$ & $60-90$ & 8076 & 4.179 & \\
\hline 0.4 & $2 * 10-2$ & $90-60$ & -8748 & 7.146 & \\
\hline \multicolumn{5}{|c|}{ Average of Fuel economy at $60 \%-60 \% \& 90 \%-90 \%$ SoC } & 4.784 \\
\hline \multicolumn{5}{|c|}{ Y intercept of $60 \%-90 \%$ and $90 \%-60 \%$ SoC } & 5.603 \\
\hline \multicolumn{5}{|l|}{$\%$ Error } & 14.62 \\
\hline$\overline{\mathrm{C} 1}$ & $\mathrm{C} 2$ & SoC & delta SoC & MPG & \\
\hline 0.5 & $11.083^{*} 10$ & $60-60$ & -0.760 & 4.790 & \\
\hline 0.5 & $11.06 * 10-2$ & 9 & -0.920 & 4.790 & \\
\hline 0.5 & $2 * 10-2$ & $60-90$ & 8270 & 4.225 & \\
\hline 0.5 & $12.966 * 10$ & $90-60$ & -8723 & 7.149 & \\
\hline \multicolumn{5}{|c|}{ Average of Fuel economy at $60 \%-60 \% \& 90 \%-90 \%$ SoC } & 4.790 \\
\hline \multicolumn{5}{|c|}{ Y intercept of $60 \%-90 \%$ and $90 \%-60 \%$ SoC } & 5.648 \\
\hline \multicolumn{5}{|l|}{$\%$ Error } & 15.19 \\
\hline
\end{tabular}

Table 26: SoC correction for HEV with auxiliary load over CBD cycle (continued in next page) 


\begin{tabular}{|c|c|c|c|c|c|}
\hline C1 & $\mathrm{C} 2$ & SoC & delta SoC & MPG & \\
\hline$\overline{0.6}$ & $8.68 * 10-2$ & $60-60$ & 0.260 & 4.808 & \\
\hline 0.6 & $8.68 * 10-2$ & $90-90$ & 0.260 & 4.808 & \\
\hline 0.6 & $1.5 * 10-2$ & $60-90$ & 8307 & 4.151 & \\
\hline 0.6 & $8.68 * 10-2$ & $90-60$ & -8748 & 7.162 & \\
\hline Average 0 & Fuel econ & omy at & $-60 \% \& 90 \%$ & $0-90 \%$ SoC & 4.808 \\
\hline Y intercep & of $60 \%-90$ & $\%$ and $s$ & $-60 \%$ SoC & & 5.617 \\
\hline$\%$ Error & & & & & $\overline{14.41}$ \\
\hline C1 & $\mathrm{C} 2$ & SoC & delta SoC & MPG & \\
\hline 0.7 & $4.21 * 10-2$ & $60-60$ & -0.100 & 4.837 & \\
\hline 0.7 & $4.21 * 10-2$ & $90-90$ & -0.100 & 4.837 & \\
\hline 0.7 & 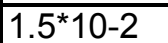 & $60-90$ & 8582 & 4.206 & \\
\hline 0.7 & $8.68 * 10-2$ & $90-60$ & -8707 & 7.176 & \\
\hline Average o & Fuel econ & omy at & $-60 \% \& 90 \%$ & $0-90 \%$ SoC & 4.837 \\
\hline Y intercep & of $60 \%-90$ & $\%$ and $s$ & $-60 \%$ SoC & & 5.680 \\
\hline$\%$ Error & & & & & 14.85 \\
\hline C1 & $\mathrm{C} 2$ & SoC & delta SoC & MPG & \\
\hline 0.8 & $4.9 * 10-2$ & $60-60$ & 62.42 & 4.901 & \\
\hline 0.8 & $5^{*} 10-2$ & $90-90$ & 61.72 & 4.901 & \\
\hline 0.8 & $0.81 * 10-2$ & $60-90$ & 8740 & 4.115 & \\
\hline 0.8 & $4 * 10-2$ & $90-60$ & -8675 & 7.204 & \\
\hline Average o & Fuel econ & omy at & $-60 \% \& 90 \%$ & $0-90 \%$ SoC & 4.901 \\
\hline Y intercep & of $60 \%-90$ & $\%$ and $s$ & $-60 \%$ SoC & & 5.665 \\
\hline$\%$ Error & & & & & 13.49 \\
\hline C1 & $\mathrm{C} 2$ & SoC & delta SoC & MPG & \\
\hline & $7.2 * 10-2$ & $60-60$ & $\overline{141.9}$ & 4.937 & \\
\hline 0.9 & $5.5^{*} 10-2$ & $90-90$ & 144.0 & 4.942 & \\
\hline 0.9 & $0.39 * 10-2$ & $60-90$ & 8736 & 4.123 & \\
\hline 0.9 & $5.5^{*} 10-2$ & $90-60$ & -8604 & 7.223 & \\
\hline Average o & Fuel econ & omy at & $-60 \%$ \&90\% & $0-90 \%$ SoC & 4.940 \\
\hline$Y$ intercep & of $60 \%-90$ & $\%$ and 5 & $-60 \%$ SoC & & 5.685 \\
\hline$\%$ Error & & & & & 13.11 \\
\hline
\end{tabular}

Table 26: SoC correction for HEV with auxiliary load over CBD cycle (continued) 


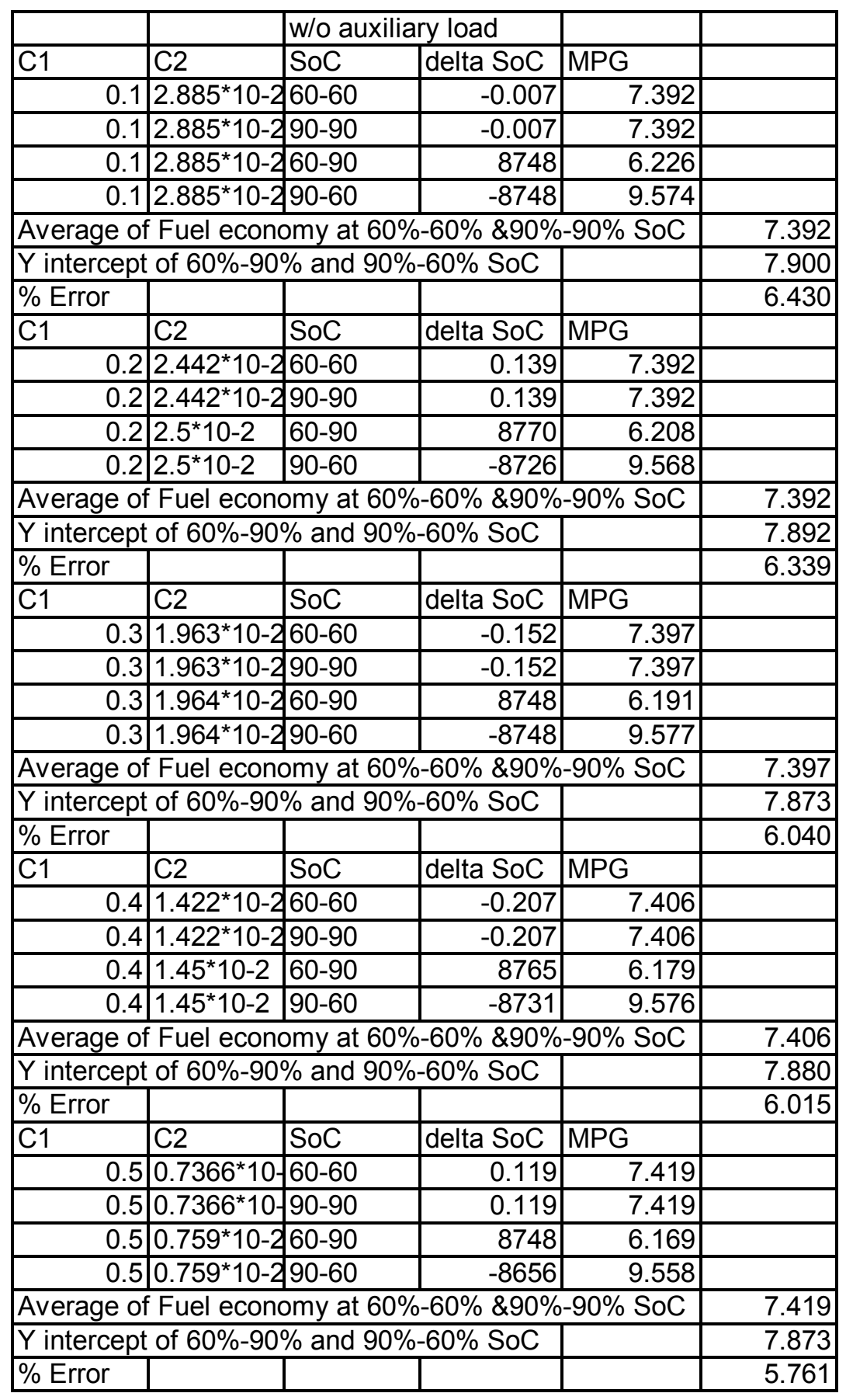

Table 27: SoC correction for HEV w/o auxiliary load over CSHVC (continued in next page) 


\begin{tabular}{|c|c|c|c|c|c|}
\hline C1 & $\mathrm{C} 2$ & SoC & delta SoC & MPG & \\
\hline$\overline{0.6}$ & $0.00071 * 1$ & $60-60$ & 457.1 & 7.360 & \\
\hline 0.6 & $0.00071^{* 1}$ & $90-90$ & 457.1 & 7.360 & \\
\hline 0.6 & $0.27 * 10-2$ & $60-90$ & 8776 & 6.168 & \\
\hline 0.6 & $0.27 * 10-2$ & $90-60$ & -6093 & 8.799 & \\
\hline Average o & Fuel econ & omy at & $-60 \% \& 90 \%$ & $-90 \%$ SoC & 7.360 \\
\hline Y intercep & of $60 \%-90$ & $\%$ and & $-60 \%$ SoC & & 7.720 \\
\hline$\%$ Error & & & & & 4.663 \\
\hline C1 & C2 & SoC & delta SoC & MPG & \\
\hline 0.7 & $2.39 * 10-2$ & $60-60$ & 1254 & 7.245 & \\
\hline 0.7 & 4 & $90-90$ & 1326 & 7.241 & \\
\hline 0.7 & $0.08 * 10-2$ & $60-90$ & 8769 & 6.193 & \\
\hline 0.7 & $5.6 * 10-2$ & $90-60$ & -7385 & 9.220 & \\
\hline & & & & & \\
\hline Average o & Fuel econ & omy at & $-60 \% \& 90 \%$ & $-90 \%$ SoC & 7.243 \\
\hline Y intercep & of $60 \%-90$ & $\%$ and & $-60 \%$ SoC & & 7.836 \\
\hline$\%$ Error & & & & & 7.569 \\
\hline C1 & $\mathrm{C} 2$ & SoC & delta SoC & MPG & \\
\hline 0.8 & $2.9 * 10-2$ & $60-60$ & 1516 & 7.220 & \\
\hline 0.8 & $3.6 * 10-2$ & $90-90$ & 1486 & 7.228 & \\
\hline 0.8 & $0.00011^{* 1}$ & $60-90$ & 10589 & 6.024 & \\
\hline 0.8 & $3.3^{*} 10-2$ & $90-60$ & -7252 & 9.181 & \\
\hline Average o & Fuel econ & omy at & $-60 \% \& 90 \%$ & $-90 \%$ SoC & 7.224 \\
\hline Y intercep & of $60 \%-90$ & $\%$ and & $-60 \%$ SoC & & 7.898 \\
\hline$\%$ Error & & & & & 8.530 \\
\hline C1 & $\mathrm{C} 2$ & SoC & delta SoC & MPG & \\
\hline 0.9 & $4 * 10-2$ & $60-60$ & - & - & \\
\hline 0.9 & $0.6803^{*} 10$ & $90-90$ & - & - & \\
\hline 0.9 & 4 4*10-2 & $60-90$ & $\overline{10412}$ & 6.275 & \\
\hline 0.9 & $4 * 10-2$ & $90-60$ & -7084 & 9.150 & \\
\hline Average 0 & Fuel econ & omy at & $-60 \% \& 90 \%$ & $-90 \%$ SoC & \\
\hline Y intercep & of $60 \%-90$ & $\%$ and & $-60 \%$ SoC & & 7.985 \\
\hline$\%$ Error & & & & & - \\
\hline
\end{tabular}

Table 27: SoC correction for HEV w/o auxiliary load over CSHVC (continued) 


\begin{tabular}{|c|c|c|c|c|c|}
\hline & & \multicolumn{2}{|c|}{ with auxiliary load } & & \\
\hline C1 & $\overline{C 2}$ & SoC & delta SoC & MPG & \\
\hline 0.1 & $116^{*} 10-2$ & $60-60$ & -11.65 & 4.964 & \\
\hline 0.1 & $76^{* 10-2}$ & $90-90$ & -17.76 & 4.930 & \\
\hline 0.1 & $3.5^{*} 10-2$ & $60-90$ & 8333 & 4.370 & \\
\hline 0.1 & $6 * 10-2$ & $90-60$ & -8974 & 5.382 & \\
\hline \multicolumn{5}{|c|}{ Average of Fuel economy at $60 \%-60 \% \& 90 \%-90 \%$ SoC } & 4.947 \\
\hline \multicolumn{5}{|c|}{ Y intercept of $60 \%-90 \%$ and $90 \%-60 \%$ SoC } & 4.857 \\
\hline \multicolumn{5}{|l|}{$\%$ Error } & -1.853 \\
\hline C1 & $\mathrm{C} 2$ & SoC & delta SoC & MPG & \\
\hline 0.2 & $116^{*} 10-2$ & $60-60$ & -10.39 & 4.955 & \\
\hline 0.2 & $76 * 10-2$ & $90-90$ & -15.78 & 4.960 & \\
\hline 0.2 & $2.9 * 10-2$ & $60-90$ & 8285 & 4.344 & \\
\hline 0.2 & $9 * 10-2$ & $90-60$ & -8881 & 5.413 & \\
\hline \multicolumn{5}{|c|}{ Average of Fuel economy at $60 \%-60 \% \& 90 \%-90 \%$ SoC } & 4.958 \\
\hline \multicolumn{5}{|c|}{$Y$ intercept of $60 \%-90 \%$ and $90 \%-60 \%$ SoC } & 4.860 \\
\hline \multicolumn{5}{|c|}{$\%$ Error } & -2.008 \\
\hline C1 & $\mathrm{C} 2$ & SoC & delta SoC & MPG & \\
\hline 0.3 & $100 * 10-2$ & $60-60$ & -10.50 & 4.939 & \\
\hline 0.3 & $75^{*} 10-2$ & $90-90$ & -13.99 & 4.924 & \\
\hline 0.3 & $2.9 * 10-2$ & $60-90$ & 8354 & 4.353 & \\
\hline 0.3 & $75^{*} 10-2$ & $90-60$ & -8762 & 5.552 & \\
\hline \multicolumn{5}{|c|}{ Average of Fuel economy at $60 \%-60 \% \& 90 \%-90 \%$ SoC } & 4.932 \\
\hline \multicolumn{5}{|c|}{ Y intercept of $60 \%-90 \%$ and $90 \%-60 \%$ SoC } & 4.938 \\
\hline \multicolumn{5}{|c|}{$\%$ Error } & 0.136 \\
\hline C1 & $\mathrm{C} 2$ & SoC & delta SoC & MPG & \\
\hline 0.4 & $115^{\star} 10-2$ & $60-60$ & -8.420 & 4.944 & \\
\hline 0.4 & $58 * 10-2$ & $90-90$ & -15.50 & 4.912 & \\
\hline 0.4 & $3.2^{*} 10-2$ & $60-90$ & 8460 & 4.238 & \\
\hline 0.4 & $6 * 10-2$ & $90-60$ & -8897 & 5.409 & \\
\hline \multicolumn{5}{|c|}{ Average of Fuel economy at $60 \%-60 \%$ \&90\%-90\% SoC } & 4.928 \\
\hline \multicolumn{5}{|c|}{ Y intercept of $60 \%-90 \%$ and $90 \%-60 \%$ SoC } & 4.809 \\
\hline \multicolumn{5}{|l|}{$\%$ Error } & -2.479 \\
\hline \multirow{5}{*}{$\frac{0.5}{0.5}$} & $\mathrm{C} 2$ & SoC & delta SoC & MPG & \\
\hline & $106^{*} 10-2$ & $60-60$ & -7.513 & 4.938 & \\
\hline & $96 * 10-2$ & $90-90$ & -7.790 & 4.934 & \\
\hline & $2.9 * 10-2$ & $60-90$ & 8493 & 4.381 & \\
\hline & $6 * 10-2$ & $90-60$ & -8870 & 5.423 & \\
\hline \multicolumn{5}{|c|}{ Average of Fuel economy at $60 \%-60 \% \& 90 \%-90 \%$ SoC } & 4.936 \\
\hline \multicolumn{5}{|c|}{ Y intercept of $60 \%-90 \%$ and $90 \%-60 \%$ SoC } & 4.891 \\
\hline \multicolumn{5}{|l|}{$\%$ Error } & -0.926 \\
\hline
\end{tabular}

Table 28: SoC correction for HEV with auxiliary load over CSHVC (continued in next page) 


\begin{tabular}{|c|c|c|c|c|c|}
\hline C1 & $\mathrm{C} 2$ & SoC & delta SoC & MPG & \\
\hline$\overline{0.6}$ & $95 * 10-2$ & $60-60$ & -5.790 & 4.936 & \\
\hline 0.6 & $71 * 10-2$ & $90-90$ & -8.129 & 4.926 & \\
\hline 0.6 & $2.4 * 10-2$ & $60-90$ & 8521 & 4.379 & \\
\hline 0.6 & $5 * 10-2$ & $90-60$ & -8861 & 5.433 & \\
\hline \multicolumn{5}{|c|}{ Average of Fuel economy at $60 \%-60 \% \& 90 \%-90 \%$ SoC } & 4.931 \\
\hline \multicolumn{5}{|c|}{ Y intercept of $60 \%-90 \%$ and $90 \%-60 \%$ SoC } & 4.896 \\
\hline \multicolumn{5}{|l|}{ \% Error } & -0.721 \\
\hline C1 & $\mathrm{C} 2$ & SoC & delta SoC & MPG & \\
\hline 0.7 & $86 * 10-2$ & $60-60$ & $\mid-3.070$ & 4.936 & \\
\hline 0.7 & $75^{*} 10-2$ & $90-90$ & -4.790 & 4.932 & \\
\hline 0.7 & $2.2^{*} 10-2$ & $60-90$ & 8609 & 4.395 & \\
\hline 0.7 & $5 * 10-2$ & $90-60$ & -8827 & 5.452 & \\
\hline \multicolumn{5}{|c|}{ Average of Fuel economy at $60 \%-60 \% \& 90 \%-90 \%$ SoC } & 4.934 \\
\hline \multicolumn{5}{|c|}{$Y$ intercept of $60 \%-90 \%$ and $90 \%-60 \%$ SoC } & 4.917 \\
\hline \multicolumn{5}{|c|}{$\%$ Error } & -0.348 \\
\hline C1 & $\mathrm{C} 2$ & SoC & delta SoC & MPG & \\
\hline 0.8 & $1.61 * 10-2$ & $60-60$ & -0.031 & 4.841 & \\
\hline 0.8 & $1.621^{* 10-2}$ & $90-90$ & -0.662 & 4.841 & \\
\hline 0.8 & $2.1 * 10-2$ & $60-90$ & 8727 & 4.418 & \\
\hline 0.8 & $4.95 * 10-2$ & $90-60$ & -8784 & 5.473 & \\
\hline \multicolumn{5}{|c|}{ Average of Fuel economy at $60 \%-60 \% \& 90 \%-90 \%$ SoC } & 4.841 \\
\hline \multicolumn{5}{|c|}{ Y intercept of $60 \%-90 \%$ and $90 \%-60 \%$ SoC } & 4.944 \\
\hline \multicolumn{5}{|l|}{$\%$ Error } & 2.080 \\
\hline $\mathrm{C} 1$ & $\mathrm{C} 2$ & SoC & delta SoC & MPG & \\
\hline 0.9 & $19.49 * 10-2$ & $60-60$ & 3.328 & 4.913 & \\
\hline 0.9 & $15.5^{*} 10-2$ & $90-90$ & 4.460 & 4.906 & \\
\hline 0.9 & $1.96 * 10-2$ & $60-90$ & 8896 & 4.441 & \\
\hline 0.9 & $4.2 * 10-2$ & $90-60$ & -8720 & 5.492 & \\
\hline \multicolumn{5}{|c|}{ Average of Fuel economy at $60 \%-60 \% \& 90 \%-90 \%$ SoC } & 4.910 \\
\hline \multicolumn{5}{|c|}{ Y intercept of $60 \%-90 \%$ and $90 \%-60 \%$ SoC } & 4.972 \\
\hline \multicolumn{5}{|l|}{$\%$ Error } & 1.251 \\
\hline
\end{tabular}

Table 28: SoC correction for HEV with auxiliary load over CSHVC (continued) 


\begin{tabular}{|c|c|c|c|c|c|}
\hline & & \multicolumn{2}{|c|}{ w/o auxiliary load } & & \\
\hline C1 & $\mathrm{C} 2$ & SoC & delta SoC & MPG & \\
\hline 0.1 & $4.892 * 10-2$ & $60-60$ & -0.017 & 5.249 & \\
\hline 0.1 & $4.892 * 10-2$ & $90-90$ & -0.017 & 5.249 & \\
\hline 0.1 & $3.4^{*} 10-2$ & $60-90$ & 8568 & 3.459 & \\
\hline 0.1 & $4.892 * 10-2$ & $90-60$ & -8249 & 6.365 & \\
\hline \multicolumn{5}{|c|}{ Average of Fuel economy at $60 \%-60 \% \& 90 \%-90 \%$ SoC } & 5.249 \\
\hline \multicolumn{4}{|c|}{$Y$ intercept of $60 \%-90 \%$ and $90 \%-60 \%$ SoC } & & 4.940 \\
\hline$\%$ Error & & & & & -6.264 \\
\hline C1 & $\mathrm{C} 2$ & SoC & delta SoC & MPG & \\
\hline 0.2 & $4.036 * 10-2$ & $60-60$ & 0.074 & 5.252 & \\
\hline 0.2 & $4.035^{*} 10-2$ & $90-90$ & -0.025 & 5.252 & \\
\hline 0.2 & $4 * 10-2$ & $60-90$ & 8745 & 3.466 & \\
\hline 0.2 & $4 * 10-2$ & $90-60$ & -8242 & 6.365 & \\
\hline \multicolumn{5}{|c|}{ Average of Fuel economy at $60 \%-60 \%$ \& $90 \%-90 \%$ SoC } & 5 \\
\hline \multicolumn{4}{|c|}{$Y$ intercept of $60 \%-90 \%$ and $90 \%-60 \%$ SoC } & & 4.958 \\
\hline \multirow{2}{*}{\begin{tabular}{|l|}
$\%$ Error \\
C1
\end{tabular}} & & & & & -5.921 \\
\hline & $\mathrm{C} 2$ & SoC & delta SoC & MPG & \\
\hline \multirow{4}{*}{$\frac{0 .}{0 .}$} & $3.024 * 10-2$ & $60-60$ & 0.051 & 5.256 & \\
\hline & $3.024 * 10-2$ & $90-90$ & 0.051 & 5.256 & \\
\hline & $3.024 * 10-2$ & $60-90$ & 8748 & 3.435 & \\
\hline & $4 * 10-2$ & $90-60$ & -8168 & 6.365 & \\
\hline \multicolumn{5}{|c|}{ Average of Fuel economy at $60 \%-60 \% \& 90 \%-90 \%$ SoC } & 5.256 \\
\hline \multicolumn{5}{|c|}{\begin{tabular}{|l|l|l|}
$Y$ intercept of $60 \%-90 \%$ and $90 \%-60 \%$ SoC & \\
\end{tabular}} & 4.950 \\
\hline \multicolumn{2}{|l|}{$\%$ Error } & & & & -6.175 \\
\hline C1 & $\mathrm{C} 2$ & SoC & delta SoC & MPG & \\
\hline 0.4 & $1.525 * 10-2$ & $60-60$ & -0.043 & 5.262 & \\
\hline $\begin{array}{r}0.4 \\
\end{array}$ & $1.525 * 10-2$ & $90-90$ & -0.043 & 5.262 & \\
\hline \multirow{2}{*}{\begin{tabular}{|l|}
0.4 \\
0.4
\end{tabular}} & $1.55^{*} 10-2$ & $60-90$ & 8742 & 3.395 & \\
\hline & $5^{*} 10-2$ & $90-60$ & -8110 & 6.365 & \\
\hline \multicolumn{5}{|c|}{ Average of Fuel economy at $60 \%-60 \% \& 90 \%-90 \%$ SoC } & 5.262 \\
\hline \multicolumn{5}{|c|}{\begin{tabular}{|l|l} 
intercept of $60 \%-90 \%$ and $90 \%-60 \%$ SoC \\
\end{tabular}} & 4.936 \\
\hline$\%$ Error & & & & & -6.611 \\
\hline C1 & $\mathrm{C} 2$ & SoC & delta SoC & MPG & \\
\hline 0.5 & $4.6 * 10-2$ & $60-60$ & 297.1 & 5.171 & \\
\hline 0.5 & $3.3^{*} 10-2$ & $90-90$ & 300.9 & 5.168 & \\
\hline \multirow{2}{*}{$\frac{0.5}{0.5}$} & $0.66 * 10-2$ & $60-90$ & 8748 & 3.381 & \\
\hline & $8.6 * 10-2$ & $90-60$ & -8161 & 6.360 & \\
\hline \multicolumn{5}{|c|}{ Average of Fuel economy at $60 \%-60 \% \& 90 \%-90 \%$ SoC } & 5.170 \\
\hline \multirow{2}{*}{\multicolumn{4}{|c|}{\begin{tabular}{|l|l|}
\multicolumn{3}{|c|}{ intercept of $60 \%-90 \%$ and $90 \%-60 \%$ SoC } \\
$\%$ Error
\end{tabular}}} & & 4.922 \\
\hline & & & & & -5.024 \\
\hline
\end{tabular}

Table 29: SoC correction for HEV w/o auxiliary load over Manhattan cycle (continued in next page) 


\begin{tabular}{|c|c|c|c|c|c|}
\hline C1 & C2 & SoC & delta SoC & MPG & \\
\hline 0.6 & $7.4 * 10-2$ & $60-60$ & 370.0 & 5.157 & \\
\hline 0.6 & $7.4 * 10-2$ & $90-90$ & 370.0 & 5.157 & \\
\hline 0.6 & \begin{tabular}{|l}
$1.7 * 10-2$ \\
\end{tabular} & $60-90$ & 9485 & 3.324 & \\
\hline 0.6 & $8.7 * 10-2$ & $90-60$ & -8093 & 6.268 & \\
\hline \multicolumn{5}{|c|}{ Average of Fuel economy at $60 \%-60 \% \& 90 \%-90 \%$ SoC } & 5.157 \\
\hline \multicolumn{5}{|c|}{ Y intercept of $60 \%-90 \%$ and $90 \%-60 \%$ SoC } & 4.913 \\
\hline$\%$ Error & & & & & -4.977 \\
\hline C1 & $\mathrm{C} 2$ & SoC & delta SoC & MPG & \\
\hline 0.7 & $13^{*} 10-2$ & $60-60$ & 412.6 & 5.158 & \\
\hline 0.7 & $11 * 10-2$ & $90-90$ & 4411.5 & 5.156 & \\
\hline 0.7 & $4.7 * 10-2$ & $60-90$ & 9306 & 3.502 & \\
\hline 0.7 & $4.7 * 10-2$ & $90-60$ & -7850 & 6.268 & \\
\hline \multicolumn{5}{|c|}{ Average of Fuel economy at $60 \%-60 \% \& 90 \%-90 \%$ SoC } & 5.157 \\
\hline \multicolumn{5}{|c|}{ Y intercept of $60 \%-90 \%$ and $90 \%-60 \%$ SoC } & 5.002 \\
\hline$\%$ Error & & & & & -3.091 \\
\hline C1 & $\mathrm{C} 2$ & SoC & delta SoC & MPG & \\
\hline 0.8 & $15^{\star} 10-2$ & $60-60$ & 442.1 & 5.158 & \\
\hline 0.8 & $16 * 10-2$ & $90-90$ & 441.3 & 5.159 & \\
\hline 0.8 & $4 * 10-2$ & $60-90$ & 9622 & 3.435 & \\
\hline 0.8 & $13 * 10-2$ & $90-60$ & -8099 & 6.268 & \\
\hline \multicolumn{5}{|c|}{ Average of Fuel economy at $60 \%-60 \% \& 90 \%-90 \%$ SoC } & 5.159 \\
\hline \multicolumn{5}{|c|}{ Y intercept of $60 \%-90 \%$ and $90 \%-60 \%$ SoC } & 4.973 \\
\hline$\%$ Error & & & & & -3.724 \\
\hline C1 & $\mathrm{C} 2$ & SoC & delta SoC & MPG & \\
\hline 0.9 & $25^{*} 10-2$ & $60-60$ & 462.0 & 5.167 & \\
\hline 0.9 & $25^{*} 10-2$ & $90-90$ & 462.0 & 5.167 & \\
\hline 0.9 & $5^{*} 10-2$ & $60-90$ & 9674 & 3.514 & \\
\hline 0.9 & $16^{*} 10-2$ & $90-60$ & -8120 & 6.268 & \\
\hline \multicolumn{5}{|c|}{ Average of Fuel economy at $60 \%-60 \% \& 90 \%-90 \%$ SoC } & 5.167 \\
\hline \multirow{2}{*}{\multicolumn{4}{|c|}{\begin{tabular}{|l|l|}
\multicolumn{3}{|c|}{ Y intercept of $60 \%-90 \%$ and $90 \%-60 \%$ SoC } \\
$\%$ Error &
\end{tabular}}} & & 5.011 \\
\hline & & & & & -3.107 \\
\hline
\end{tabular}

Table 29: SoC correction for HEV w/o auxiliary load over Manhattan cycle (continued) 


\begin{tabular}{|c|c|c|c|c|c|}
\hline & & with & iliary load & & \\
\hline C1 & C2 & SoC & delta SoC & MPG & \\
\hline 0.1 & $109 * 10-2$ & $60-60$ & -0.550 & 2.695 & \\
\hline 0.1 & $109 * 10-2$ & $90-90$ & -0.550 & 2.695 & \\
\hline 0.1 & $4 * 10-2$ & $60-90$ & 8303 & 2.181 & \\
\hline 0.1 & $8 * 10-2$ & $90-60$ & -8922 & 3.545 & \\
\hline Average of & f Fuel econ & omy at & $-60 \% \& 90 \%$ & $0-90 \%$ SoC & 2.695 \\
\hline Y intercept & of $60 \%-90$ & $\%$ and & $-60 \%$ SoC & & 2.839 \\
\hline$\%$ Error & & & & & 5.055 \\
\hline C1 & $\mathrm{C} 2$ & SoC & delta SoC & MPG & \\
\hline 0.2 & $99.2 * 10-2$ & $60-60$ & -0.094 & 2.687 & \\
\hline 0.2 & $99.2 * 10-2$ & $90-90$ & -0.094 & 2.687 & \\
\hline 0.2 & $3.9 * 10-2$ & $60-90$ & 8353 & 2.180 & \\
\hline 0.2 & $9 * 10-2$ & $90-60$ & -8878 & 3.543 & \\
\hline Average of & f Fuel econ & omy at & $-60 \% \& 90 \%$ & $-90 \%$ SoC & 2.687 \\
\hline Y intercept & of $60 \%-90$ & $\%$ and & $-60 \%$ SoC & & 2.841 \\
\hline$\%$ Error & & & & & 5.407 \\
\hline C1 & $\mathrm{C} 2$ & SoC & delta SoC & MPG & \\
\hline 0.3 & $86.8 * 10-2$ & $60-60$ & 0.045 & 2.680 & \\
\hline 0.3 & $86.6^{*} 10-2$ & $90-90$ & -0.064 & 2.680 & \\
\hline 0.3 & $3.9^{*} 10-2$ & $60-90$ & 8418 & 2.183 & \\
\hline 0.3 & $9 * 10-2$ & $90-60$ & -8858 & 3.543 & \\
\hline Average of & f Fuel econ & omy at & -60\% \&90\% & $0-90 \%$ SoC & 2.680 \\
\hline Y intercept & of $60 \%-90$ & $\%$ and & $-60 \%$ SoC & & 2.846 \\
\hline$\%$ Error & & & & & 5.826 \\
\hline C1 & $\mathrm{C} 2$ & SoC & delta SoC & MPG & \\
\hline 0.4 & $72.7 * 10-2$ & $60-60$ & 0.016 & 2.675 & \\
\hline 0.4 & $72.67^{*} 10-2$ & $90-90$ & -0.002 & 2.675 & \\
\hline 0.4 & $3 * 10-2$ & $60-90$ & 8369 & 2.170 & \\
\hline 0.4 & $8.3^{*} 10-2$ & $90-60$ & -8846 & 3.546 & \\
\hline Average of & f Fuel econ & omy at & $-60 \% \& 90 \%$ & $0-90 \%$ SoC & 2.675 \\
\hline Y intercept & of $60 \%-90$ & $\%$ and & $-60 \%$ SoC & & 2.839 \\
\hline$\%$ Error & & & & & 5.777 \\
\hline$\overline{C 1}$ & $\mathrm{C} 2$ & SoC & delta SoC & MPG & \\
\hline 0.5 & $61.54 * 10-2$ & $60-60$ & -2.815 & 2.673 & \\
\hline 0.5 & $61.55^{*} 10-2$ & $90-90$ & 2.188 & 2.673 & \\
\hline 0.5 & $3 * 10-2$ & $60-90$ & 8462 & 2.175 & \\
\hline 0.5 & $8.3^{*} 10-2$ & $90-60$ & -8823 & 3.549 & \\
\hline Average of & fuel econ & omy at & $-60 \% \& 90 \%$ & $0-90 \%$ SoC & 2.673 \\
\hline Y intercept & of $60 \%-90$ & $\%$ and & $-60 \%$ SoC & & 2.848 \\
\hline \begin{tabular}{|l|}
$\%$ Error \\
\end{tabular} & & & & & 6.135 \\
\hline
\end{tabular}

Table 30: SoC correction for HEV with auxiliary load over Manhattan cycle (continued in next page) 


\begin{tabular}{|c|c|c|c|c|c|}
\hline C1 & $\mathrm{C} 2$ & SoC & delta SoC & MPG & \\
\hline$\overline{0.6}$ & $46 * 10-2$ & $60-60$ & 0.020 & 2.671 & \\
\hline 0.6 & $466^{* 10-2}$ & $90-90$ & 0.020 & 2.671 & \\
\hline$\overline{0.6}$ & $3 * 10-2$ & $60-90$ & 8555 & 2.181 & \\
\hline 0.6 & $4.5 * 10-2$ & $90-60$ & -8857 & 3.557 & \\
\hline \multicolumn{5}{|c|}{ Average of Fuel economy at $60 \%-60 \% \& 90 \%-90 \%$ SoC } & 2.671 \\
\hline \multicolumn{5}{|c|}{ Y intercept of $60 \%-90 \%$ and $90 \%-60 \%$ SoC } & 2.857 \\
\hline$\%$ Error & & & & & $\overline{6.514}$ \\
\hline C1 & $\mathrm{C} 2$ & SoC & delta SoC & MPG & \\
\hline 0.7 & $32 * 10-2$ & $60-60$ & $\mid-0.052$ & 2.670 & \\
\hline 0.7 & $31 * 10-2$ & $90-90$ & -0.171 & 2.670 & \\
\hline 0.7 & $2 * 10-2$ & $60-90$ & 8576 & 2.171 & \\
\hline 0.7 & $2 * 10-2$ & $90-60$ & -8920 & 3.574 & \\
\hline \multicolumn{5}{|c|}{ Average of Fuel economy at $60 \%-60 \% \& 90 \%-90 \%$ SoC } & 2.670 \\
\hline \multicolumn{5}{|c|}{$\mathrm{Y}$ intercept of $60 \%-90 \%$ and $90 \%-60 \%$ SoC } & 2.859 \\
\hline$\%$ Error & & & & & 6.603 \\
\hline$\overline{C 1}$ & $\mathrm{C} 2$ & SoC & delta SoC & MPG & \\
\hline 0.8 & $9.11 * 10-2$ & $60-60$ & -0.476 & 2.663 & \\
\hline 0.8 & $10 * 10-2$ & $90-90$ & 0.426 & 2.663 & \\
\hline 0.8 & $1 * 10-2$ & $60-90$ & 8722 & 2.158 & \\
\hline 0.8 & $1 * 10-2$ & $90-60$ & -8762 & 3.565 & \\
\hline \multicolumn{5}{|c|}{ Average of Fuel economy at $60 \%-60 \% \& 90 \%-90 \%$ SoC } & 2.663 \\
\hline \multicolumn{5}{|c|}{ Y intercept of $60 \%-90 \%$ and $90 \%-60 \%$ SoC } & 2.860 \\
\hline$\%$ Error & & & & & 6.885 \\
\hline C1 & $\mathrm{C} 2$ & SoC & delta SoC & MPG & \\
\hline 0.9 & $12 * 10-2$ & $60-60$ & 25.15 & 2.676 & \\
\hline 0.9 & $10 * 10-2$ & $90-90$ & 26.61 & 2.675 & \\
\hline 0.9 & $1.4 * 10-2$ & $60-90$ & 8976 & 2.170 & \\
\hline 0.9 & $1.9 * 10-2$ & $90-60$ & -8591 & 3.553 & \\
\hline \multicolumn{5}{|c|}{ Average of Fuel economy at $60 \%-60 \% \& 90 \%-90 \%$ SoC } & 2.676 \\
\hline \multirow{2}{*}{\multicolumn{4}{|c|}{\begin{tabular}{|l|l|l|}
\multicolumn{3}{|c|}{ Y intercept of $60 \%-90 \%$ and $90 \%-60 \%$ SoC } \\
$\%$ Error & &
\end{tabular}}} & & 2.877 \\
\hline & & & & & 6.994 \\
\hline
\end{tabular}

Table 30: SoC correction for HEV with auxiliary load over Manhattan cycle (continued) 


\begin{tabular}{|c|c|c|c|c|c|}
\hline \multirow[b]{2}{*}{$\overline{\mathrm{C} 1}$} & \multirow[b]{2}{*}{$\mathrm{C} 2$} & \multicolumn{2}{|c|}{ w/o auxiliary load } & \multirow[b]{2}{*}{ MPG } & \\
\hline & & SoC & delta SoC & & \\
\hline 0.1 & $3.65 * 10-2$ & $60-60$ & -0.887 & 5.960 & \\
\hline 0.1 & $3.65^{*} 10-2$ & $90-90$ & -0.887 & 5.960 & \\
\hline 0.1 & $3.65^{*} 10-2$ & $60-90$ & 8747 & 5.232 & \\
\hline 0.1 & $3.65 * 10-2$ & $90-60$ & -8749 & 7.455 & \\
\hline \multicolumn{5}{|c|}{ Average of Fuel economy at $60 \%-60 \% \& 90 \%-90 \%$ SoC } & 5.960 \\
\hline \multicolumn{4}{|c|}{$Y$ intercept of $60 \%-90 \%$ and $90 \%-60 \%$ SoC } & & 4.661 \\
\hline \multirow{2}{*}{\begin{tabular}{|l|}
$\%$ Error \\
C1
\end{tabular}} & & & & & -27.87 \\
\hline & $\mathrm{C} 2$ & SoC & delta SoC & MPG & \\
\hline & $2.86 * 10-2$ & $60-60$ & 0.311 & 5.967 & \\
\hline & $2.86 * 10-2$ & $90-90$ & 0.311 & 5.967 & \\
\hline & $2.9 * 10-2$ & $60-90$ & 8764 & 5.187 & \\
\hline & $2.9^{*} 10-2$ & $90-60$ & -8732 & 7.460 & \\
\hline \multicolumn{5}{|c|}{ Average of Fuel economy at $60 \%-60 \% \& 90 \%-90 \%$ SoC } & 5.967 \\
\hline \multicolumn{4}{|c|}{$Y$ intercept of $60 \%-90 \%$ and $90 \%-60 \%$ SoC } & & 4.650 \\
\hline \multirow{2}{*}{\begin{tabular}{|l|}
$\%$ Error \\
C1
\end{tabular}} & & & & & -28.32 \\
\hline & $\mathrm{C} 2$ & SoC & delta SoC & MPG & \\
\hline 0.3 & $2.158 * 10-2$ & $60-60$ & -0.194 & 5.985 & \\
\hline 0.3 & $2.158 * 10-2$ & $90-90$ & -0.194 & 5.985 & \\
\hline 0.3 & $2.158^{*} 10-2$ & $60-90$ & 8748 & 5.165 & \\
\hline 0.3 & $2.158 * 10-2$ & $90-60$ & -8744 & 7.484 & \\
\hline \multicolumn{5}{|c|}{ Average of Fuel economy at $60 \%-60 \% \& 90 \%-90 \%$ SoC } & 5.985 \\
\hline \multicolumn{4}{|c|}{ Y intercept of $60 \%-90 \%$ and $90 \%-60 \%$ SoC } & & 4.709 \\
\hline$\%$ Error & & & & & -27.11 \\
\hline C1 & $\mathrm{C} 2$ & SoC & delta SoC & MPG & \\
\hline 0.4 & $1.603 * 10-2$ & $60-60$ & 0.153 & 6.015 & \\
\hline 0.4 & $1.603^{*} 10-2$ & $90-90$ & 0.153 & 6.015 & \\
\hline \multirow{2}{*}{\begin{tabular}{|l|}
0.4 \\
0.4
\end{tabular}} & $1.6 * 10-2$ & $60-90$ & 8742 & 5.170 & \\
\hline & $1.6^{*} 10-2$ & $90-60$ & -8727 & 7.514 & \\
\hline \multicolumn{5}{|c|}{ Average of Fuel economy at $60 \%-60 \% \& 90 \%-90 \%$ SoC } & 6.015 \\
\hline \multicolumn{4}{|c|}{ Y intercept of $60 \%-90 \%$ and $90 \%-60 \%$ SoC } & & 4.732 \\
\hline$\%$ Error & & & & & -27.11 \\
\hline C1 & $\mathrm{C} 2$ & SoC & delta SoC & MPG & \\
\hline \multirow{2}{*}{\begin{tabular}{|l|}
0.5 \\
0.5
\end{tabular}} & $1.144^{*} 10-2$ & $60-60$ & -0.607 & 6.059 & \\
\hline & $1.144 * 10-2$ & $90-90$ & -0.607 & 6.059 & \\
\hline 0.5 & $1.144 * 10-2$ & $60-90$ & 8720 & 5.195 & \\
\hline 0.5 & $1.144 * 10-2$ & $90-60$ & -8637 & 7.554 & \\
\hline Average of & Fuel econs & omy at & $-60 \% \& 90 \%$ & $90 \%$ SoC & 6.059 \\
\hline Y intercept & of $60 \%-90$ & $\%$ and & $-60 \%$ SoC & & 4.740 \\
\hline$\%$ Error & & & & & -27.83 \\
\hline
\end{tabular}

Table 31: SoC correction for HEV w/o auxiliary load over UDDS cycle (continued in next page) 


\begin{tabular}{|c|c|c|c|c|c|}
\hline C1 & $\mathrm{C} 2$ & SoC & delta SoC & MPG & \\
\hline$\overline{0.6}$ & $0.71 * 10-2$ & $60-60$ & -0.293 & 6.121 & \\
\hline 0.6 & $0.71 * 10-2$ & $90-90$ & -0.293 & 6.121 & \\
\hline 0.6 & $0.78 * 10-2$ & $60-90$ & 8762 & 5.228 & \\
\hline 0.6 & $0.46 * 10-2$ & $90-60$ & -8420 & 7.607 & \\
\hline \multicolumn{5}{|c|}{ Average of Fuel economy at $60 \%-60 \% \& 90 \%-90 \%$ SoC } & 6.121 \\
\hline \multicolumn{5}{|c|}{\begin{tabular}{|l|l|l}
$Y$ intercept of $60 \%-90 \%$ and $90 \%-60 \%$ SoC & \\
\end{tabular}} & 4.758 \\
\hline$\%$ Error & & & & & -28.65 \\
\hline C1 & C2 & SoC & delta SoC & MPG & \\
\hline 0.7 & $0.011^{* 10-2}$ & $60-60$ & 36.54 & 6.215 & \\
\hline 0.7 & $0.011 * 10-2$ & $90-90$ & 36.54 & 6.215 & \\
\hline 0.7 & $0.44 * 10-2$ & $60-90$ & 8710 & 5.279 & \\
\hline 0.7 & $1.4^{*} 10-2$ & $90-60$ & -6738 & 7.186 & \\
\hline \multicolumn{5}{|c|}{ Average of Fuel economy at $60 \%-60 \% \& 90 \%-90 \%$ SoC } & 6.215 \\
\hline \multirow{2}{*}{\multicolumn{5}{|c|}{\begin{tabular}{|l|l|l|}
\multicolumn{3}{|c|}{ Y intercept of $60 \%-90 \%$ and $90 \%-60 \%$ SoC } \\
$\%$ Error & &
\end{tabular}}} & 4.798 \\
\hline & & & & & -29.52 \\
\hline $\mathrm{C} 1$ & $\mathrm{C} 2$ & SoC & delta SoC & MPG & \\
\hline 0.8 & $0.18 * 10-2$ & $60-60$ & - & - & \\
\hline 0.8 & $1.099 * 10-2$ & $90-90$ & - & - & \\
\hline 0.8 & $0.18 * 10-2$ & $60-90$ & 8733 & 5.330 & \\
\hline 0.8 & $37 * 10-2$ & $90-60$ & -6930 & 7.144 & \\
\hline \multicolumn{6}{|c|}{ Average of Fuel economy at $60 \%-60 \% \& 90 \%-90 \%$ SoC } \\
\hline \multicolumn{5}{|c|}{\begin{tabular}{|l|l|} 
Y intercept of $60 \%-90 \%$ and $90 \%-60 \%$ SoC \\
\end{tabular}} & 4.878 \\
\hline$\%$ Error & & & & & - \\
\hline C1 & $\mathrm{C} 2$ & SoC & delta SoC & MPG & \\
\hline 0.9 & $1.504 * 10-2$ & $60-60$ & - & - & \\
\hline 0.9 & $0.6803^{*} 10$ & $90-90$ & - & - & \\
\hline 0.9 & $10 * 10-2$ & $60-90$ & 9488 & 5.337 & \\
\hline 0.9 & $16 * 10-2$ & $90-60$ & -6763 & 7.124 & \\
\hline \multicolumn{5}{|c|}{ Average of Fuel economy at $60 \%-60 \% \& 90 \%-90 \%$ SoC } & \\
\hline \multirow{2}{*}{\multicolumn{4}{|c|}{\begin{tabular}{|l|l|l|}
\multicolumn{3}{|c|}{ intercept of $60 \%-90 \%$ and $90 \%-60 \%$ SoC } \\
$\%$ Error &
\end{tabular}}} & & 4.933 \\
\hline & & & & & - \\
\hline
\end{tabular}

Table 31:SoC correction for HEV w/o auxiliary load over UDDS cycle (continued) 


\begin{tabular}{|c|c|c|c|c|c|}
\hline & & with & iliary laod & & \\
\hline C1 & $\overline{C 2}$ & SoC & delta SoC & MPG & \\
\hline 0.1 & $55^{*} 10-2$ & $60-60$ & -24.54 & 4.700 & \\
\hline 0.1 & $55^{*} 10-2$ & $90-90$ & -24.54 & 4.700 & \\
\hline 0.1 & $3^{*} 10-2$ & $60-90$ & 8249 & 4.164 & \\
\hline 0.1 & $5 * 10-2$ & $90-60$ & -9027 & 5.206 & \\
\hline Average of & Fuel eco & omy at & $-60 \% \& 90 \%$ & $-90 \%$ SoC & 4.700 \\
\hline Y intercept & of $60 \%-9$ & $\%$ and & $-60 \%$ SoC & & 6.343 \\
\hline$\%$ Error & & & & & 25.91 \\
\hline C1 & $\mathrm{C} 2$ & SoC & delta SoC & MPG & \\
\hline 0.2 & $55^{*} 10-2$ & $60-60$ & -21.80 & 4.693 & \\
\hline 0.2 & $55^{*} 10-2$ & $90-90$ & -21.80 & 4.693 & \\
\hline 0.2 & $3.5^{\star} 10-2$ & $60-90$ & 8381 & 4.203 & \\
\hline 0.2 & $5 * 10-2$ & $90-60$ & -8996 & 5.130 & \\
\hline Average of & f Fuel eco & omy at & $-60 \% \& 90 \%$ & b-90\% SoC & 4.693 \\
\hline Y intercept & of $60 \%-9$ & $\%$ and & $-60 \%$ SoC & & 6.326 \\
\hline$\%$ Error & & & & & 25.81 \\
\hline C1 & $\mathrm{C} 2$ & SoC & delta SoC & MPG & \\
\hline 0.3 & $75^{*} 10-2$ & $60-60$ & -14.00 & 4.729 & \\
\hline 0.3 & $75^{*} 10-2$ & $90-90$ & -14.00 & 4.729 & \\
\hline 0.3 & $3.5^{*} 10-2$ & $60-90$ & 8430 & 4.222 & \\
\hline 0.3 & $5 * 10-2$ & $90-60$ & -8965 & 5.226 & \\
\hline Average of & Fuel eco & omy at & $-60 \% \& 90 \%$ & $0-90 \%$ SoC & 4.729 \\
\hline Y intercept & of $60 \%-9$ & $\%$ and & $-60 \%$ SoC & & 6.325 \\
\hline$\%$ Error & & & & & 25.23 \\
\hline$\overline{\mathrm{C} 1}$ & $\mathrm{C} 2$ & SoC & delta SoC & MPG & \\
\hline 0.4 & $75^{*} 10-2$ & $60-60$ & -12.00 & 4.732 & \\
\hline 0.4 & $75^{*} 10-2$ & $90-90$ & -12.00 & 4.732 & \\
\hline 0.4 & $3.5^{*} 10-2$ & $60-90$ & 8478 & 4.246 & \\
\hline 0.4 & $5 * 10-2$ & $90-60$ & -8933 & 5.244 & \\
\hline Average of & Fuel eco & omy at & $-60 \% \& 90 \%$ & $-90 \%$ SoC & 4.732 \\
\hline Y intercept & of $60 \%-9$ & $\%$ and & $-60 \%$ SoC & & 6.343 \\
\hline$\%$ Error & & & & & 25.40 \\
\hline$\overline{C 1}$ & $\mathrm{C} 2$ & SoC & delta SoC & MPG & \\
\hline 0.5 & $55^{*} 10-2$ & $60-60$ & -13.63 & 4.721 & \\
\hline 0.5 & $55^{*} 10-2$ & $90-90$ & -13.63 & 4.721 & \\
\hline 0.5 & $3 * 10-2$ & $60-90$ & 8489 & 4.250 & \\
\hline 0.5 & $3 * 10-2$ & $90-60$ & -9008 & 5.260 & \\
\hline Average of & f Fuel eco & omy at & $-60 \%$ \&90\% & b-90\% SoC & 4.721 \\
\hline $\mathrm{Y}$ intercept & of $60 \%-9$ & $\%$ and & $-60 \%$ SoC & & 6.380 \\
\hline$\%$ Error & & & & & 26.01 \\
\hline
\end{tabular}

Table 32: SoC correction for HEV with auxiliary load over UDDS cycle (continued in next page) 


\begin{tabular}{|c|c|c|c|c|c|}
\hline C1 & $\mathrm{C} 2$ & SoC & delta SoC & MPG & \\
\hline$\overline{0.6}$ & $50 * 10-2$ & $60-60$ & -12.00 & 4.743 & \\
\hline 0.6 & $40 * 10-2$ & $90-90$ & -15.00 & 4.734 & \\
\hline 0.6 & $2.2 * 10-2$ & $60-90$ & 8481 & 4.255 & \\
\hline 0.6 & $2.2 * 10-2$ & $90-60$ & -9015 & 5.292 & \\
\hline \multicolumn{5}{|c|}{ Average of Fuel economy at $60 \%-60 \% \& 90 \%-90 \%$ SoC } & 4.739 \\
\hline \multicolumn{5}{|c|}{\begin{tabular}{|l|l|l}
$Y$ intercept of $60 \%-90 \%$ and $90 \%-60 \%$ SoC & \\
\end{tabular}} & 6.441 \\
\hline$\%$ Error & & & & & 26.43 \\
\hline C1 & C2 & SoC & delta SoC & MPG & \\
\hline 0.7 & $100 * 10-2$ & $60-60$ & -4.500 & 4.821 & \\
\hline 0.7 & $100 * 10-2$ & $90-90$ & -4.500 & 4.821 & \\
\hline 0.7 & $1.8 * 10-2$ & $60-90$ & 8558 & 4.292 & \\
\hline 0.7 & $2.1^{*} 10-2$ & $90-60$ & -8917 & 5.326 & \\
\hline \multicolumn{5}{|c|}{ Average of Fuel economy at $60 \%-60 \% \& 90 \%-90 \%$ SoC } & 4.821 \\
\hline \multirow{2}{*}{\multicolumn{5}{|c|}{\begin{tabular}{|l|l|l|}
\multicolumn{3}{|c|}{ Y intercept of $60 \%-90 \%$ and $90 \%-60 \%$ SoC } \\
$\%$ Error & &
\end{tabular}}} & 6.354 \\
\hline & & & & & 24.13 \\
\hline $\mathrm{C} 1$ & $\mathrm{C} 2$ & SoC & delta SoC & MPG & \\
\hline 0.8 & $2.76 * 10-2$ & $60-60$ & -0.223 & 4.757 & \\
\hline 0.8 & $58 * 10-2$ & $90-90$ & -5.170 & 4.829 & \\
\hline 0.8 & $2.5^{*} 10-2$ & $60-90$ & 8753 & 4.370 & \\
\hline 0.8 & $11 * 10-2$ & $90-60$ & -8773 & 5.381 & \\
\hline \multicolumn{5}{|c|}{ Average of Fuel economy at $60 \%-60 \% \& 90 \%-90 \%$ SoC } & 4.793 \\
\hline \multicolumn{5}{|c|}{ Y intercept of $60 \%-90 \%$ and $90 \%-60 \%$ SoC } & 6.341 \\
\hline$\%$ Error & & & & & 24.42 \\
\hline C1 & $\mathrm{C} 2$ & SoC & delta SoC & MPG & \\
\hline 0.9 & $8.75^{*} 10-2$ & $60-60$ & -0.865 & 4.836 & \\
\hline 0.9 & $8.6 * 10-2$ & $90-90$ & -0.121 & 4.836 & \\
\hline 0.9 & $2.5^{*} 10-2$ & $60-90$ & 9058 & 4.428 & \\
\hline 0.9 & $8.6 * 10-2$ & $90-60$ & -8749 & 5.420 & \\
\hline \multicolumn{5}{|c|}{ Average of Fuel economy at $60 \%-60 \% \& 90 \%-90 \%$ SoC } & 4.836 \\
\hline \multirow{2}{*}{\multicolumn{4}{|c|}{\begin{tabular}{|l|l|l|}
\multicolumn{3}{|c|}{ intercept of $60 \%-90 \%$ and $90 \%-60 \%$ SoC } \\
$\%$ Error & &
\end{tabular}}} & & 6.380 \\
\hline & & & & & 24.20 \\
\hline
\end{tabular}

Table 32: SoC correction for HEV with auxiliary load over UDDS cycle (continued) 


\begin{tabular}{|c|c|c|c|c|c|}
\hline & & withou & xiliary load & & \\
\hline C1 & C2 & SoC & delta SoC & MPG & \\
\hline 0.1 & $3.252 * 10-2$ & $60-60$ & -0.205 & 5.241 & \\
\hline 0.1 & $3.252 * 10-2$ & $90-90$ & -0.205 & 5.241 & \\
\hline 0.1 & $3.24 * 10-2$ & $60-90$ & 8736 & 4.284 & \\
\hline 0.1 & $3.24^{*} 10-2$ & $90-60$ & -8760 & 6.833 & \\
\hline Average of & f Fuel econc & omy at & $-60 \% \& 90 \%$ & $0-90 \%$ SoC & 5.241 \\
\hline Y intercept & of $60 \%-90$ & $\%$ and & $-60 \%$ SoC & & 5.557 \\
\hline$\%$ Error & & & & & 5.681 \\
\hline C1 & $\mathrm{C} 2$ & SoC & delta SoC & MPG & \\
\hline 0.2 & $2.9 * 10-2$ & $60-60$ & 0.311 & 5.242 & \\
\hline 0.2 & $2.9 * 10-2$ & $90-90$ & 0.311 & 5.242 & \\
\hline 0.2 & $2.9 * 10-2$ & $60-90$ & 8748 & 4.283 & \\
\hline 0.2 & $2.9^{*} 10-2$ & $90-60$ & -8746 & 6.831 & \\
\hline Average of & f Fuel econo & omy at & $-60 \% \& 90 \%$ & $-90 \%$ SoC & 5.242 \\
\hline Y intercept & of $60 \%-90$ & $\%$ and & $-60 \%$ SoC & & 5.557 \\
\hline$\%$ Error & & & & & 5.672 \\
\hline C1 & $\mathrm{C} 2$ & SoC & delta SoC & MPG & \\
\hline 0.3 & $2.539 * 10-2$ & $60-60$ & -0.492 & 5.244 & \\
\hline 0.3 & $2.539 * 10-2$ & $90-90$ & -0.492 & 5.244 & \\
\hline 0.3 & $2.539 * 10-2$ & $60-90$ & 8747 & 4.284 & \\
\hline 0.3 & $2.539 * 10-2$ & $90-60$ & -8745 & 5.832 & \\
\hline Average of & Fuel econo & omy at & -60\% \&90\% & $0-90 \%$ SoC & 5.244 \\
\hline Y intercept & of $60 \%-90^{\circ}$ & $\%$ and & $-60 \%$ SoC & & 5.058 \\
\hline$\%$ Error & & & & & -3.675 \\
\hline C1 & $\mathrm{C} 2$ & SoC & delta SoC & MPG & \\
\hline 0.4 & $1.777^{*} 10-2$ & $60-60$ & -0.734 & 5.249 & \\
\hline 0.4 & $2.168 * 10-2$ & 9 & 0.284 & 5.249 & \\
\hline 0.4 & $2.16^{*} 10-2$ & $60-90$ & 8736 & 4.286 & \\
\hline 0.4 & $2.168 * 10-2$ & $90-60$ & -8738 & 6.833 & \\
\hline Average of & Fuel econo & omy at & $-60 \% \& 90 \%$ & $0-90 \%$ SoC & 5.249 \\
\hline Y intercept & of $60 \%-90^{\circ}$ & $\%$ and & $-60 \%$ SoC & & 5.559 \\
\hline$\%$ Error & & & & & 5.583 \\
\hline$\overline{C 1}$ & $\mathrm{C} 2$ & SoC & delta SoC & MPG & \\
\hline 0.5 & $1.777^{*} 10-2$ & $60-60$ & -0.734 & 5.249 & \\
\hline 0.5 & $1.777^{*} 10-2$ & $90-90$ & -0.734 & 5.249 & \\
\hline 0.5 & $1.777^{*} 10-2$ & $60-90$ & 8745 & 4.287 & \\
\hline 0.5 & $1.76^{*} 10-2$ & $90-60$ & -8746 & 6.839 & \\
\hline Average of & Fuel econo & omy at & $-60 \% \& 90 \%$ & $0-90 \%$ SoC & 5.249 \\
\hline Y intercept & of $60 \%-90^{\circ}$ & $\%$ and & $-60 \%$ SoC & & 5.563 \\
\hline \begin{tabular}{|l|}
$\%$ Error \\
\end{tabular} & & & & & 5.644 \\
\hline
\end{tabular}

Table 33: SoC correction for HEV w/o auxiliary load over UDDS1 cycle (continued in next page) 


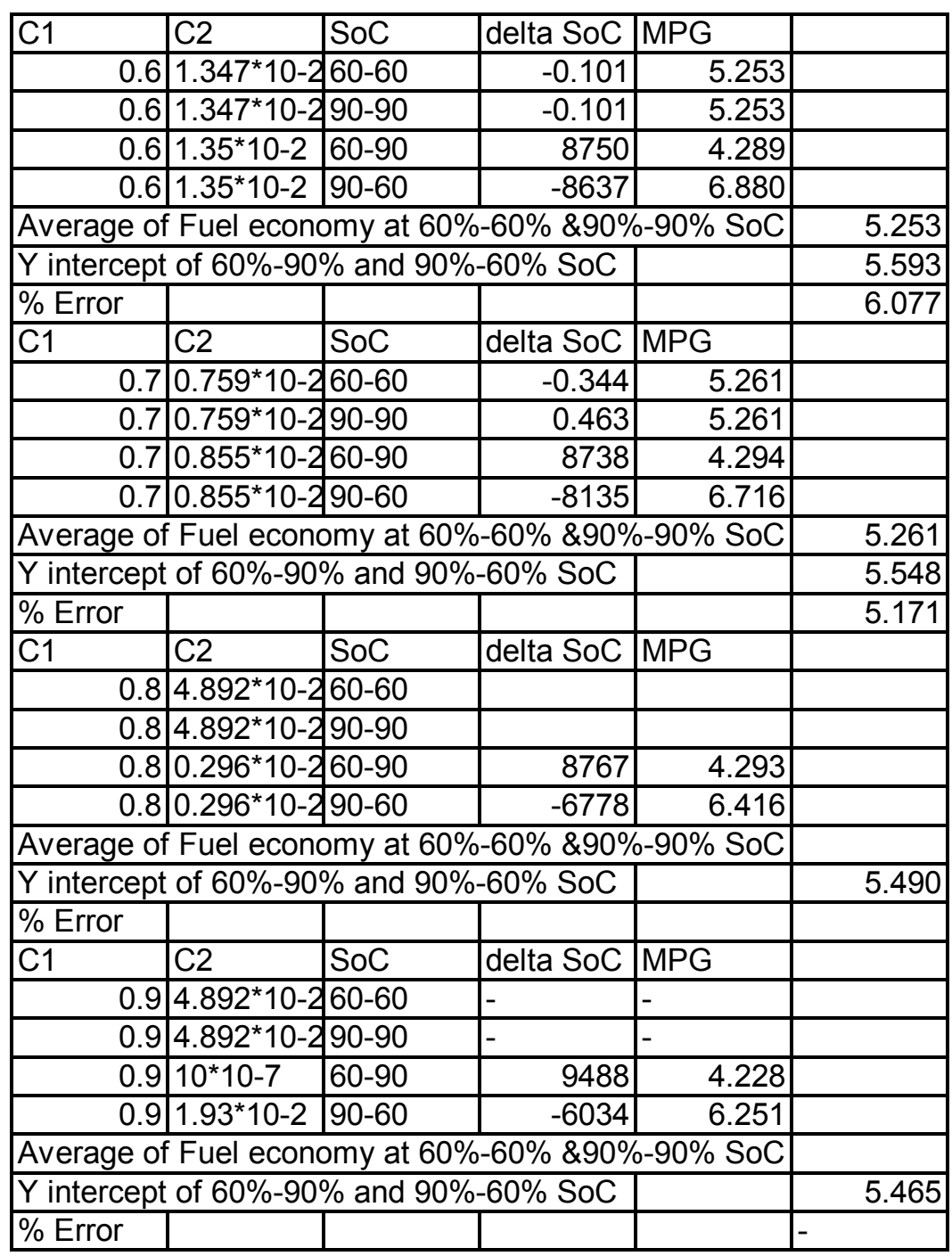

Table 33: SoC correction for HEV w/o auxiliary load over UDDS1 cycle (continued) 


\begin{tabular}{|c|c|c|c|c|c|}
\hline & & with & iliary load & & \\
\hline C1 & C2 & SoC & delta SoC & MPG & \\
\hline 0.1 & $4.345^{* 10-2}$ & $60-60$ & -0.713 & 3.721 & \\
\hline 0.1 & $4.343^{*} 10-2$ & $90-90$ & 2.620 & 3.721 & \\
\hline 0.1 & $4.3^{*} 10-2$ & $60-90$ & 8717 & 3.237 & \\
\hline 0.1 & $4.3 * 10-2$ & $90-60$ & -8775 & 4.416 & \\
\hline Average of & f Fuel econ & omy at & $-60 \% \& 90 \%$ & $0-90 \%$ SoC & 3.721 \\
\hline Y intercept & of $60 \%-90$ & $\%$ and & $-60 \%$ SoC & & 3.816 \\
\hline$\%$ Error & & & & & 2.501 \\
\hline C1 & $\mathrm{C} 2$ & SoC & delta SoC & MPG & \\
\hline 0.2 & $3.89 * 10-2$ & $60-60$ & 1.740 & 3.721 & \\
\hline 0.2 & $3.88^{*} 10-2$ & $90-90$ & -0.964 & 3.721 & \\
\hline 0.2 & $3.9 * 10-2$ & $60-90$ & 8756 & 3.235 & \\
\hline 0.2 & $3.88 * 10-2$ & $90-60$ & -8749 & 4.414 & \\
\hline Average of & Fuel econo & omy at & $-60 \% \& 90 \%$ & $-90 \%$ SoC & 3.721 \\
\hline Y intercept & of $60 \%-90$ & $\%$ and & $-60 \%$ SoC & & 3.825 \\
\hline$\%$ Error & & & & & 2.711 \\
\hline C1 & $\mathrm{C} 2$ & SoC & delta SoC & MPG & \\
\hline 0.3 & $3.415 * 10-2$ & $60-60$ & 0.493 & 3.722 & \\
\hline 0.3 & $3.41 * 10-2$ & $90-90$ & -0.761 & 3.722 & \\
\hline 0.3 & $3.45^{*} 10-2$ & $60-90$ & 8774 & 3.234 & \\
\hline 0.3 & $3.53^{*} 10-2$ & $90-60$ & -8657 & 4.394 & \\
\hline Average of & Fuel econc & omy at & -60\% \&90\% & $0-90 \%$ SoC & 3.722 \\
\hline $\mathrm{Y}$ intercept & of $60 \%-90$ & $\%$ and & $-60 \%$ SoC & & 3.818 \\
\hline$\%$ Error & & & & & 2.512 \\
\hline C1 & $\mathrm{C} 2$ & SoC & delta SoC & MPG & \\
\hline 0.4 & $2.933 * 10-2$ & $60-60$ & -0.986 & \begin{tabular}{l|}
3.723 \\
\end{tabular} & \\
\hline 0.4 & $2.932 * 10-2$ & $90-90$ & -0.736 & 3.723 & \\
\hline 0.4 & $2.933 * 10-2$ & $60-90$ & 8747 & 3.236 & \\
\hline 0.4 & $2.932 * 10-2$ & $90-60$ & -8749 & 4.403 & \\
\hline Average of & Fuel econo & omy at & $-60 \% \& 90 \%$ & $0-90 \%$ SoC & 3.723 \\
\hline Y intercept & of $60 \%-90^{\circ}$ & $\%$ and & $-60 \%$ SoC & & 3.819 \\
\hline$\%$ Error & & & & & 2.524 \\
\hline$\overline{C 1}$ & $\mathrm{C} 2$ & SoC & delta SoC & MPG & \\
\hline 0.5 & $2.443^{*} 10-2$ & $60-60$ & -0.416 & 3.725 & \\
\hline 0.5 & $2.443^{*} 10-2$ & $90-90$ & -0.676 & 3.725 & \\
\hline 0.5 & $2.45 * 10-2$ & $60-90$ & 8755 & 3.236 & \\
\hline 0.5 & $2.45^{*} 10-2$ & $90-60$ & -8741 & 4.396 & \\
\hline Average of & Fuel econo & omy at & $-60 \% \& 90 \%$ & $0-90 \%$ SoC & 3.725 \\
\hline Y intercept & of $60 \%-90^{\circ}$ & $\%$ and & $-60 \%$ SoC & & 3.816 \\
\hline \begin{tabular}{|l|}
$\%$ Error \\
\end{tabular} & & & & & 2.395 \\
\hline
\end{tabular}

Table 34: SoC correction for HEV with auxiliary load over UDDS1 cycle (continued in next page) 


\begin{tabular}{|c|c|c|c|c|c|}
\hline C1 & $\mathrm{C} 2$ & SoC & delta SoC & MPG & \\
\hline$\overline{0.6}$ & $1.935^{*} 10-2$ & $60-60$ & -0.510 & 3.727 & \\
\hline 0.6 & $1.937^{* 10}-2$ & $90-90$ & -0.282 & 3.727 & \\
\hline 0.6 & $1.935^{*} 10-2$ & $60-90$ & 8747 & 3.238 & \\
\hline 0.6 & $1.937^{*} 10-2$ & $90-60$ & -8748 & 4.401 & \\
\hline \multicolumn{5}{|c|}{ Average of Fuel economy at $60 \%-60 \% \& 90 \%-90 \%$ SoC } & 3.727 \\
\hline \multicolumn{5}{|c|}{$Y$ intercept of $60 \%-90 \%$ and $90 \%-60 \%$ SoC } & 3.820 \\
\hline$\%$ Error & & & & & 2.422 \\
\hline C1 & $\mathrm{C} 2$ & SoC & delta SoC & MPG & \\
\hline 0.7 & $1.393^{*} 10-2$ & $60-60$ & -0.197 & 3.730 & \\
\hline 0.7 & $1.481 * 10-2$ & $90-90$ & -0.079 & 3.730 & \\
\hline 0.7 & $1.374 * 10-2$ & $60-90$ & 8717 & 3.241 & \\
\hline 0.7 & $1.48 * 10-2$ & $90-60$ & -8749 & 4.406 & \\
\hline \multicolumn{5}{|c|}{ Average of Fuel economy at $60 \%-60 \% \& 90 \%-90 \%$ SoC } & 3.730 \\
\hline \multicolumn{5}{|c|}{ Y intercept of $60 \%-90 \%$ and $90 \%-60 \%$ SoC } & 3.822 \\
\hline$\%$ Error & & & & & 2.417 \\
\hline C1 & $\mathrm{C} 2$ & SoC & delta SoC & MPG & \\
\hline 0.8 & $0.749 * 10-2$ & $60-60$ & -0.394 & 3.735 & \\
\hline 0.8 & $0.905^{*} 10-2$ & $90-90$ & 0.967 & 3.734 & \\
\hline 0.8 & $0.749 * 10-2$ & $60-90$ & 8737 & 3.243 & \\
\hline 0.8 & $0.905^{*} 10-2$ & $90-60$ & -8740 & 4.402 & \\
\hline \multicolumn{5}{|c|}{ Average of Fuel economy at $60 \%-60 \% \& 90 \%-90 \%$ SoC } & 3.735 \\
\hline \multicolumn{5}{|c|}{ Y intercept of $60 \%-90 \%$ and $90 \%-60 \%$ SoC } & 3.822 \\
\hline$\%$ Error & & & & & 2.300 \\
\hline C1 & C2 & SoC & delta SoC & MPG & \\
\hline 0.9 & $0.66 * 10-2$ & $60-60$ & -191.8 & 3.748 & \\
\hline 0.9 & $0.091 * 10-2$ & $90-90$ & 30.96 & 3.578 & \\
\hline 0.9 & $0.16 * 10-2$ & $60-90$ & 8771 & 3.242 & \\
\hline 0.9 & $0.6 * 10-2$ & $90-60$ & -6830 & 4.256 & \\
\hline \multicolumn{5}{|c|}{ Average of Fuel economy at $60 \%-60 \% \& 90 \%-90 \%$ SoC } & 3.663 \\
\hline \multirow{2}{*}{\multicolumn{4}{|c|}{\begin{tabular}{|l|l|l|}
\multicolumn{3}{|c|}{ intercept of $60 \%-90 \%$ and $90 \%-60 \%$ SoC } \\
$\%$ Error &
\end{tabular}}} & & 3.812 \\
\hline & & & & & 3.909 \\
\hline
\end{tabular}

Table 34: SoC correction for HEV with auxiliary load over UDDS1 cycle (continued) 


\begin{tabular}{|c|c|c|c|c|c|}
\hline & & $w / o$ & ilary load & & \\
\hline C1 & C2 & SoC & delta SoC & MPG & \\
\hline 0.1 & $3.71 * 10-2$ & $60-60$ & -0.650 & 5.435 & \\
\hline 0.1 & $3.71 * 10-2$ & $90-90$ & -0.650 & 5.435 & \\
\hline 0.1 & $3.1^{*} 10-2$ & $60-90$ & 8585 & \begin{tabular}{l|}
4.521 \\
\end{tabular} & \\
\hline 0.1 & $3.71 * 10-2$ & $90-60$ & -8749 & 7.011 & \\
\hline Average of & f Fuel econ & omy at & $-60 \% \& 90 \%$ & $0-90 \%$ SoC & 5.435 \\
\hline Y intercept & of $60 \%-90$ & $\%$ and & $-60 \%$ SoC & & 5.754 \\
\hline$\%$ Error & & & & & 5.547 \\
\hline C1 & $\mathrm{C} 2$ & SoC & delta SoC & MPG & \\
\hline 0.2 & $2.74 * 10-2$ & $60-60$ & -0.426 & 5.437 & \\
\hline 0.2 & $2.74 * 10-2$ & $90-90$ & -0.426 & 5.437 & \\
\hline 0.2 & $3.1^{*} 10-2$ & $60-90$ & 8839 & 4.501 & \\
\hline 0.2 & $3.17 * 10-2$ & $90-60$ & -8641 & 6.988 & \\
\hline Average of & f Fuel econ & omy at & $-60 \% \& 90 \%$ & $-90 \%$ SoC & 5.437 \\
\hline Y intercept & of $60 \%-90$ & $\%$ and & $-60 \%$ SoC & & 5.759 \\
\hline$\%$ Error & & & & & 5.583 \\
\hline C1 & $\mathrm{C} 2$ & SoC & delta SoC & MPG & \\
\hline 0.3 & $1.814 * 10-2$ & $60-60$ & 0.316 & 5.445 & \\
\hline 0.3 & $1.814^{*} 10-2$ & $90-90$ & 0.316 & 5.445 & \\
\hline 0.3 & $1.814^{*} 10-2$ & $60-90$ & 8746 & 4.504 & \\
\hline 0.3 & $1.814 * 10-2$ & $90-60$ & -8741 & 7.029 & \\
\hline Average of & f Fuel econ & omy at & -60\% \&90\% & $0-90 \%$ SoC & 5.445 \\
\hline Y intercept & of $60 \%-90$ & $\%$ and & $-60 \%$ SoC & & 5.767 \\
\hline$\%$ Error & & & & & 5.582 \\
\hline$\overline{\mathrm{C} 1}$ & $\mathrm{C} 2$ & SoC & delta SoC & MPG & \\
\hline 0.4 & $1.178 * 10-2$ & $60-60$ & 0.979 & 5.457 & \\
\hline 0.4 & $1.178 * 10-2$ & 9 & 0.979 & 5.457 & \\
\hline 0.4 & $1.182 * 10-2$ & $60-90$ & 8734 & 4.508 & \\
\hline 0.4 & $1.14 * 10-2$ & $90-60$ & -8735 & 7.047 & \\
\hline Average of & f Fuel econ & omy at & $-60 \% \& 90 \%$ & $0-90 \%$ SoC & 5.457 \\
\hline Y intercept & of $60 \%-90$ & $\%$ and & $-60 \%$ SoC & & 5.777 \\
\hline$\%$ Error & & & & & 5.546 \\
\hline$\overline{C 1}$ & $\mathrm{C} 2$ & SoC & delta SoC & MPG & \\
\hline 0.5 & $0.751^{*} 10-2$ & $60-60$ & 0.207 & 5.471 & \\
\hline 0.5 & $0.751 * 10-2$ & 9 & 0.207 & 5.471 & \\
\hline 0.5 & $0.793^{*} 10-2$ & $60-90$ & 8716 & 4.515 & \\
\hline 0.5 & $0.621 * 10-2$ & $90-60$ & -8688 & 7.061 & \\
\hline Average of & fuel econ & omy at & $-60 \% \& 90 \%$ & $0-90 \%$ SoC & 5.471 \\
\hline Y intercept & of $60 \%-90$ & $\%$ and & $-60 \%$ SoC & & 5.790 \\
\hline \begin{tabular}{|l|}
$\%$ Error \\
\end{tabular} & & & & & 5.509 \\
\hline
\end{tabular}

Table 35: SoC correction for HEV w/o auxiliary load over UDDS2 cycle (continued in next page) 


\begin{tabular}{|c|c|c|c|c|c|}
\hline C1 & $\mathrm{C} 2$ & SoC & delta SoC & MPG & \\
\hline$\overline{0.6}$ & $0.395^{*} 10-2$ & $60-60$ & -0.686 & 5.487 & \\
\hline 0.6 & $0.395 * 10-2$ & 90 & -0.686 & 5.487 & \\
\hline 0.6 & $0.53 * 10-2$ & $60-90$ & 8772 & 4.517 & \\
\hline 0.6 & $0.395^{* 10-2}$ & $90-60$ & -7168 & 6.732 & \\
\hline \multicolumn{5}{|c|}{ Average of Fuel economy at $60 \%-60 \% \& 90 \%-90 \%$ SoC } & 5.487 \\
\hline \multirow{2}{*}{\multicolumn{5}{|c|}{\begin{tabular}{|l|l|l|l|} 
Y intercept of $60 \%-90 \%$ and $90 \%-60 \%$ SoC & \\
$\%$ Error &
\end{tabular}}} & 5.736 \\
\hline & & & & & 4.341 \\
\hline C1 & C2 & SoC & delta SoC & MPG & \\
\hline 0.7 & $0.001^{* 10-2}$ & $60-60$ & 25.72 & 5.501 & \\
\hline 0.7 & $0.001 * 10-2$ & $90-90$ & 25.72 & 5.501 & \\
\hline 0.7 & $0.31 * 10-2$ & $60-90$ & 8748 & 4.526 & \\
\hline 0.7 & $16 * 10-2$ & $90-60$ & -7004 & 6.622 & \\
\hline \multicolumn{5}{|c|}{ Average of Fuel economy at $60 \%-60 \% \& 90 \%-90 \%$ SoC } & 5.501 \\
\hline \multirow{2}{*}{\multicolumn{5}{|c|}{\begin{tabular}{|l|l|l|}
\multicolumn{3}{|c|}{ Y intercept of $60 \%-90 \%$ and $90 \%-60 \%$ SoC } \\
$\%$ Error & &
\end{tabular}}} & 5.690 \\
\hline & & & & & 3.322 \\
\hline $\mathrm{C} 1$ & $\mathrm{C} 2$ & SoC & delta SoC & MPG & \\
\hline 0.8 & $1 * 10-2$ & $60-60$ & - & - & \\
\hline 0.8 & $4.892 * 10-2$ & $90-90$ & - & - & \\
\hline 0.8 & $0.133^{*} 10-2$ & $60-90$ & 8791 & 4.531 & \\
\hline 0.8 & $11 * 10-2$ & $90-60$ & -6857 & 6.596 & \\
\hline \multicolumn{5}{|c|}{ Average of Fuel economy at $60 \%-60 \% \& 90 \%-90 \%$ SoC } & \\
\hline \multicolumn{5}{|c|}{ Y intercept of $60 \%-90 \%$ and $90 \%-60 \%$ SoC } & 5.691 \\
\hline \multirow{2}{*}{ \% Error } & & & & & - \\
\hline & $\mathrm{C} 2$ & SoC & delta SoC & MPG & \\
\hline 0.9 & $4.892 * 10-2$ & $60-60$ & - & - & \\
\hline 0.9 & $4.892 * 10-2$ & $90-90$ & - & - & \\
\hline 0.9 & $10 * 10-7$ & $60-90$ & 9489 & 4.479 & \\
\hline 0.9 & $16 * 10-2$ & $90-60$ & -6837 & 6.596 & \\
\hline \multicolumn{5}{|c|}{ Average of Fuel economy at $60 \%-60 \% \& 90 \%-90 \%$ SoC } & \\
\hline \multirow{2}{*}{\multicolumn{4}{|c|}{\begin{tabular}{|l|l|l|}
\multicolumn{3}{|c|}{ Y intercept of $60 \%-90 \%$ and $90 \%-60 \%$ SoC } \\
$\%$ Error & & \\
\end{tabular}}} & & 5.709 \\
\hline & & & & & - \\
\hline
\end{tabular}

Table 35: SoC correction for HEV w/o auxiliary load over UDDS2 cycle (continued) 


\begin{tabular}{|c|c|c|c|c|c|}
\hline & & \multicolumn{2}{|c|}{ with auxiliary load } & & \\
\hline$\overline{C 1}$ & $\mathrm{C} 2$ & SoC & delta SoC & MPG & \\
\hline 0.1 & $95^{*} 10-2$ & $60-60$ & -14.21 & 3.981 & \\
\hline 0.1 & $95^{*} 10-2$ & $90-90$ & -14.21 & 3.981 & \\
\hline 0.1 & $5.3 * 10-2$ & $60-90$ & 8485 & 3.501 & \\
\hline 0.1 & $9.5 * 10-2$ & $90-60$ & -8891 & 4.633 & \\
\hline \multicolumn{5}{|c|}{ Average of Fuel economy at $60 \%-60 \% \& 90 \%-90 \%$ SoC } & 3.981 \\
\hline \multicolumn{4}{|c|}{ Y intercept of $60 \%-90 \%$ and $90 \%-60 \%$ SoC } & & 4.054 \\
\hline$\%$ Error & & & & & 1.796 \\
\hline C1 & $\mathrm{C} 2$ & SoC & delta SoC & MPG & \\
\hline $\begin{array}{r}0.2 \\
02\end{array}$ & $86 * 10-2$ & $60-60$ & -13.95 & 3.974 & \\
\hline \multirow{2}{*}{\begin{tabular}{|l}
0.2 \\
0.2
\end{tabular}} & $86^{*} 10-2$ & $90-90$ & -13.95 & 3.974 & \\
\hline & $5.3^{*} 10-2$ & $60-90$ & 8515 & 3.503 & \\
\hline 0.2 & $9.5 * 10-2$ & $90-60$ & -8875 & 4.633 & \\
\hline \multicolumn{5}{|c|}{ Average of Fuel economy at $60 \%-60 \% \& 90 \%-90 \%$ SoC } & 3.974 \\
\hline \multicolumn{4}{|c|}{$Y$ intercept of $60 \%-90 \%$ and $90 \%-60 \%$ SoC } & & 4.056 \\
\hline \multirow{2}{*}{\begin{tabular}{|l|}
$\%$ Error \\
C1
\end{tabular}} & & & & & 2.029 \\
\hline & $\mathrm{C} 2$ & SoC & delta SoC & MPG & \\
\hline & $89 * 10-2$ & $60-60$ & -11.80 & 3.973 & \\
\hline & $89 * 10-2$ & $90-90$ & -11.80 & 3.973 & \\
\hline \begin{tabular}{|l}
0.3 \\
0.3 \\
\end{tabular} & $4.7^{*} 10-2$ & $60-90$ & 8517 & 3.496 & \\
\hline 0.3 & $4.7 * 10-2$ & $90-60$ & -8979 & 4.639 & \\
\hline \multicolumn{5}{|c|}{ Average of Fuel economy at $60 \%-60 \% \& 90 \%-90 \%$ SoC } & 3.973 \\
\hline \multicolumn{4}{|c|}{$Y$ intercept of $60 \%-90 \%$ and $90 \%-60 \%$ SoC } & & 4.052 \\
\hline \% Error & & & & & 1.959 \\
\hline C1 & $\mathrm{C} 2$ & SoC & delta SoC & MPG & \\
\hline & $73 * 10-2$ & $60-60$ & -12.32 & 3.968 & \\
\hline & $62 * 10-2$ & $90-90$ & -14.51 & 3.966 & \\
\hline & $4.7^{*} 10-2$ & $60-90$ & 8550 & 3.501 & \\
\hline 0.4 & $5.7^{*} 10-2$ & $90-60$ & -8910 & 4.637 & \\
\hline \multicolumn{5}{|c|}{ Average of Fuel economy at $60 \%-60 \% \& 90 \%-90 \%$ SoC } & 3.967 \\
\hline \multicolumn{4}{|c|}{ Y intercept of $60 \%-90 \%$ and $90 \%-60 \%$ SoC } & & 4.057 \\
\hline$\%$ Error & & & & & 2.226 \\
\hline $\mathrm{C} 1$ & $\mathrm{C} 2$ & SoC & delta SoC & MPG & \\
\hline 0.5 & $73 * 10-2$ & $60-60$ & -10.27 & 3.761 & \\
\hline 0.5 & $62 * 10-2$ & $90-90$ & -12.10 & 3.761 & \\
\hline 0.5 & $3.9 * 10-2$ & $60-90$ & 8549 & 3.498 & \\
\hline 0.5 & $3.9 * 10-2$ & $90-60$ & -8947 & 4.644 & \\
\hline Average of & f Fuel eco & omy at & $-60 \% \& 90 \%$ & $0-90 \%$ SoC & 3.761 \\
\hline Y intercept & of $60 \%-9$ & $\%$ and & $-60 \%$ SoC & & 4.058 \\
\hline$\%$ Error & & & & & 7.319 \\
\hline
\end{tabular}

Table 36: SoC correction for HEV with auxiliary load over UDDS2 cycle (continued in next page) 


\begin{tabular}{|c|c|c|c|c|c|}
\hline C1 & $\mathrm{C} 2$ & SoC & delta SoC & MPG & \\
\hline$\overline{0.6}$ & $42 * 10-2$ & $60-60$ & -14.29 & 3.968 & \\
\hline 0.6 & $43^{* 10-2}$ & $90-90$ & -13.95 & 3.969 & \\
\hline 0.6 & $3.9 * 10-2$ & $60-90$ & 8592 & 3.503 & \\
\hline 0.6 & $3.9 * 10-2$ & $90-60$ & -8904 & 4.646 & \\
\hline \multicolumn{5}{|c|}{ Average of Fuel economy at $60 \%-60 \% \& 90 \%-90 \%$ SoC } & 3.969 \\
\hline \multicolumn{5}{|c|}{\begin{tabular}{|l|l|l}
$Y$ intercept of $60 \%-90 \%$ and $90 \%-60 \%$ SoC & \\
\end{tabular}} & 4.064 \\
\hline$\%$ Error & & & & & 2.357 \\
\hline C1 & $\mathrm{C} 2$ & SoC & delta SoC & MPG & \\
\hline 0.7 & $42 * 10-2$ & $60-60$ & $\mid-10.71$ & 3.595 & \\
\hline 0.7 & $443^{* 10-2}$ & $90-90$ & -10.47 & 3.973 & \\
\hline 0.7 & $\begin{array}{l}1.9 * 10-2 \\
\end{array}$ & $60-90$ & 8552 & 3.499 & \\
\hline 0.7 & $1.9 * 10-2$ & $90-60$ & -8944 & 4.663 & \\
\hline \multicolumn{5}{|c|}{ Average of Fuel economy at $60 \%-60 \% \& 90 \%-90 \%$ SoC } & 3.784 \\
\hline \multirow{2}{*}{\multicolumn{5}{|c|}{\begin{tabular}{|l|l|l|}
\multicolumn{3}{|c|}{ Y intercept of $60 \%-90 \%$ and $90 \%-60 \%$ SoC } \\
$\%$ Error & &
\end{tabular}}} & 4.068 \\
\hline & & & & & 6.979 \\
\hline $\mathrm{C} 1$ & $\mathrm{C} 2$ & SoC & delta SoC & MPG & \\
\hline 0.8 & $1 * 10-2$ & $60-60$ & - & - & \\
\hline 0.8 & $4.892 * 10-2$ & $90-90$ & - & - & \\
\hline 0.8 & $1.9 * 10-2$ & $60-90$ & 8679 & 3.503 & \\
\hline 0.8 & $1.9 * 10-2$ & $90-60$ & -8817 & 4.66 & \\
\hline \multicolumn{5}{|c|}{ Average of Fuel economy at $60 \%-60 \% \& 90 \%-90 \%$ SoC } & \\
\hline \multicolumn{5}{|c|}{ Y intercept of $60 \%-90 \%$ and $90 \%-60 \%$ SoC } & 4.077 \\
\hline \multirow{2}{*}{ \% Error } & & & & & - \\
\hline & $\mathrm{C} 2$ & SoC & delta SoC & MPG & \\
\hline 0.9 & $4.892 * 10-2$ & $60-60$ & - & - & \\
\hline 0.9 & $4.892 * 10-2$ & $90-90$ & - & - & \\
\hline 0.9 & $1.9 * 10-2$ & $60-90$ & 8879 & 3.503 & \\
\hline 0.9 & $1.9 * 10-2$ & $90-60$ & -8617 & 4.651 & \\
\hline \multicolumn{5}{|c|}{ Average of Fuel economy at $60 \%-60 \% \& 90 \%-90 \%$ SoC } & \\
\hline \multirow{2}{*}{\multicolumn{4}{|c|}{\begin{tabular}{|l|l|l|}
\multicolumn{3}{|c|}{ intercept of $60 \%-90 \%$ and $90 \%-60 \%$ SoC } \\
$\%$ Error &
\end{tabular}}} & & 4.086 \\
\hline & & & & & - \\
\hline
\end{tabular}

Table 36: SoC correction for HEV with auxiliary load over UDDS2 cycle (continued) 